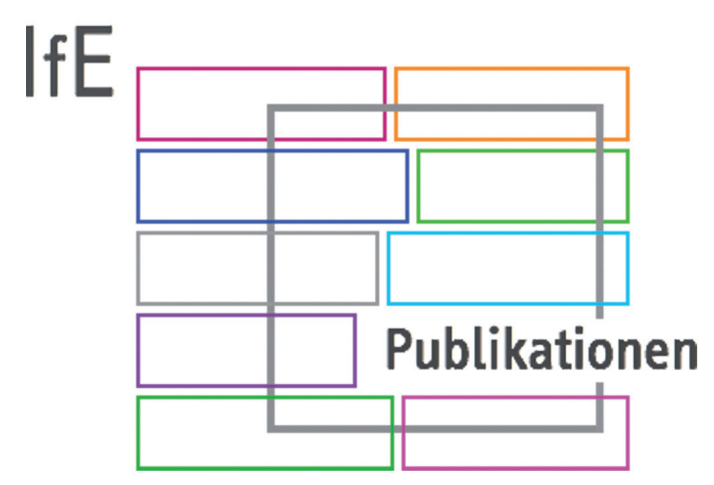

Göttinger Beiträge zur erziehungswissenschaftlichen

Forschung

37

\title{
Multiprofessionalität in der inklusiven Schule: Eine empirische Studie zur Kooperation von Lehrkräften und Schulbegleiter/innen
}

\section{(Göttinger Schulbegleitungsstudie GötS)}

Karina Meyer 

Veröffentlicht als

Göttinger Beiträge zur erziehungswissenschaftlichen Forschung, 37

Institut für Erziehungswissenschaft 2017

Karina Meyer

Multiprofessionalität in der inklusiven Schule:

Eine empirische Studie zur Kooperation von

Lehrkräften und Schulbegleiter/innen

(Göttinger Schulbegleitungsstudie GötS)

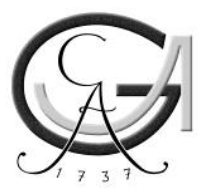

Georg-August-Universität Göttingen 2017

Dieses Werk ist lizenziert unter einer

Creative Commons Namensnennung - Weitergabe unter gleichen Bedingungen 4.0 International Lizenz.

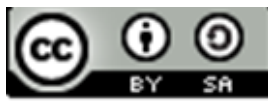


Bibliographische Information der Deutschen Nationalbibliothek

Die Deutsche Nationalbibliothek verzeichnet diese Publikation in der Deutschen Nationalbibliographie; detaillierte bibliographische Daten sind im Internet über $<$ http://dnb.dnb.de $>$ abrufbar.

Anschrift des Autors

Karina Meyer

Institut für Erziehungswissenschaft

Georg-August-Universität Göttingen

Waldweg 26

37073 Göttingen

Satz und Layout: Karina Meyer

eISSN: 2198-2384

DOI: 10.17875/gup2017-1029 


\section{Vorwort \& Danksagung}

Die Idee zu der Abschlussarbeit, die Sie in den Händen halten, ist aus einem Gespräch mit einer Studentin der Sozialen Arbeit entstanden, die zu dem Zeitpunkt selbst als Schulbegleiterin tätig war. Im Vorfeld der Studie habe ich mit verschiedenen Praxisvertreter/innen gesprochen und dabei eine hohe Bereitschaft, über das Thema Schulbegleitung zu informieren, und ein großes Interesse an meinem Forschungsvorhaben erlebt. Dafür möchte ich an dieser Stelle einen herzlichen Dank aussprechen! Die Zuständigen der Träger, der Ämter und der Fachstelle für Diagnostik haben mir per Mail, Telefon und in teilweise mehrstündigen Gesprächen wertvolle Auskünfte zu verschiedenen regionalen und überregionalen Aspekten von Schulbegleitung gegeben und die Weiterleitung meiner Umfrage an die Göttinger Schulbegleiter/innen ermöglicht. Insbesondere danke ich Frau Mindt, Frau Feußner, Frau Draing, Frau Welter und Frau Auspurg für ihre Unterstützung des Projekts. Ohne die Schulbegleiter/innen, die sich die Zeit für die Beantwortung des Fragebogens genommen haben, wäre die Studie nicht möglich gewesen. Ich danke allen Teilnehmer/innen der Befragung, besonders auch für die zahlreichen zusätzlichen Hinweise und Kommentare zu Schulbegleitung, die mir zugesandt wurden.

Einen entscheidenden Beitrag zum Gelingen der vorliegenden Arbeit haben Dr. Sonja Nonte und Prof. Dr. Ariane S. Willems geleistet, die sich viel Zeit genommen haben, die Studie mit ihrer fachlichen Expertise zu begleiten, und die mich in allen Phasen der Arbeit umfassend unterstützt haben. Vielen Dank dafür! Ein herzlicher Dank auch an die gesamte Mitarbeiterschaft der Arbeitsbereiche von Prof. Dr. Ariane S. Willems und Prof. Dr. Tobias C. Stubbe, an Alexander Schreiber, Mosa A. Essel, alle Korrekturleser/innen und die Mitarbeiter/innen des Learning Resources Center.

Dass bei den Arbeitsbedingungen von Schulbegleiter/innen und bei der Konzeption der Maßnahme Schulbegleitung Veränderungsbedarf besteht, wurde in den Vorgesprächen zur Studie und in den Kommentaren der befragten Schulbegleiter/innen mehr als deutlich. Mir bleibt zu hoffen, dass die Ergebnisse meiner Arbeit einen Beitrag dazu leisten, Schulbegleitung besser in ein Gesamtkonzept inklusiver Beschulung einzubetten.

Für Nachfragen, Hinweise oder bei Interesse an einer Zusammenarbeit zum Thema Schulbegleitung bin ich erreichbar unter kmeyer@uni-goettingen.de und unter karina.meyer@mail.de.

Göttingen, 30.11.2016

Karina Meyer 


\section{Inhalt}

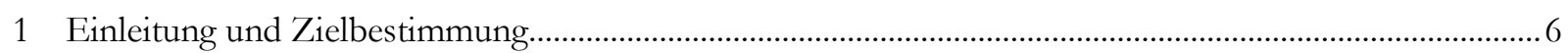

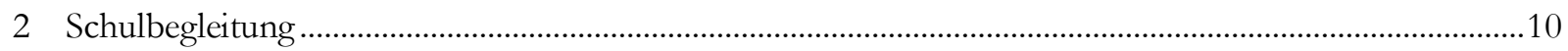

2.1 Schulbegleitung als Maßnahme der Eingliederungshilfe ..........................................................10

2.1.1 Rechtliche Grundlagen........................................................................................11

2.1.2 Bewilligungs- und Durchführungspraxis in Stadt und Landkreis Göttingen ..................13

2.1.3 Zentrale Diskussionspunkte zu Schulbegleitung im Kontext von Inklusion...................14

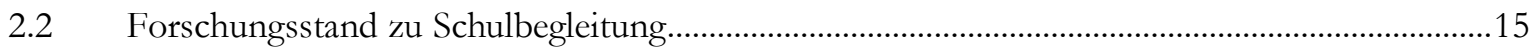

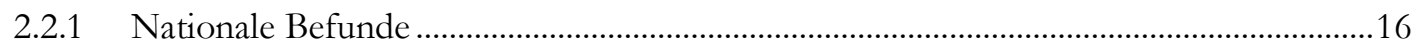

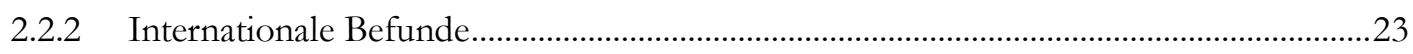

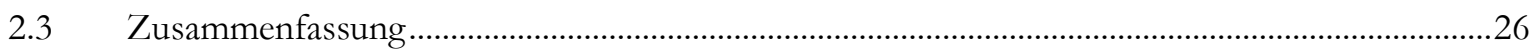

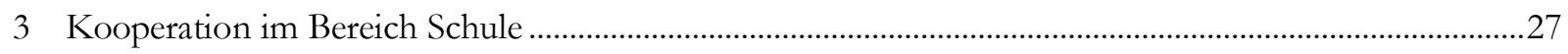

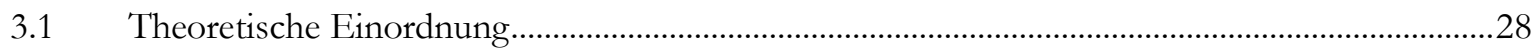

3.1.1 Begriffsbestimmung..........................................................................................28

3.1.2 Rahmenbedingungen und Besonderheiten schulischer Kooperation .............................29

3.1.3 Konzeptualisierungen und Modelle schulischer Kooperation............................................33

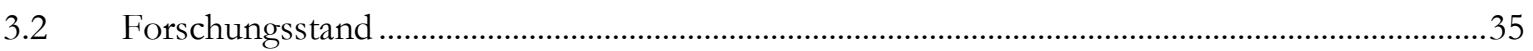

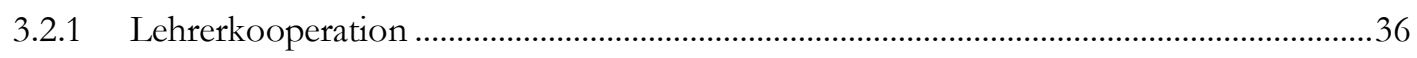

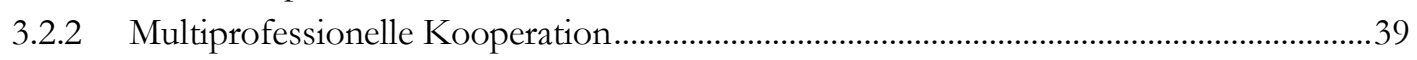

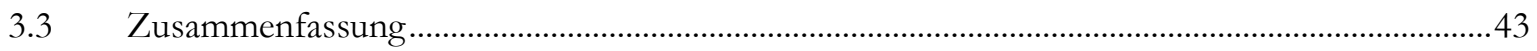

4 Zusammenfassung und Forschungsdesiderate …………………………………….................................... 44

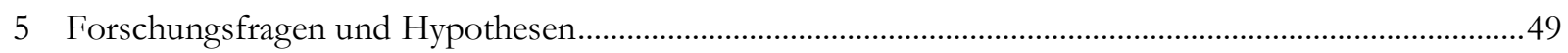

5.1 Rahmendaten zu Schulbegleitung in Stadt und Landkreis Göttingen..........................................49

$5.2 \quad$ Kooperation mit Schulbegleiter/innen ................................................................................... 50

6 Datengrundlage und methodisches Vorgehen ..........................................................................................52

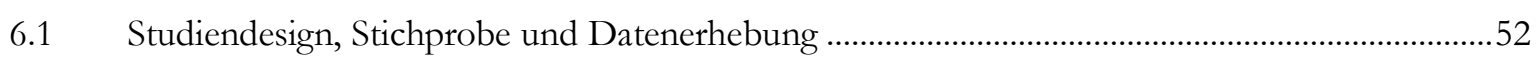

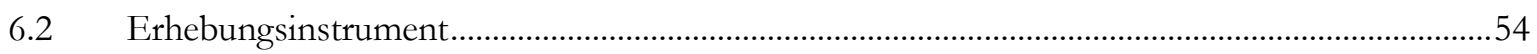

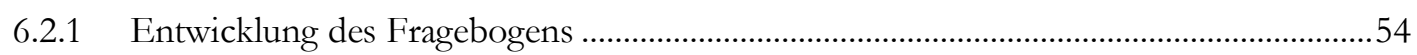

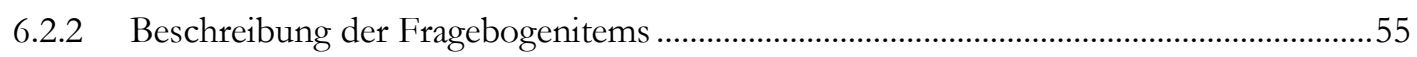

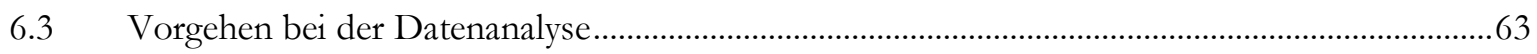

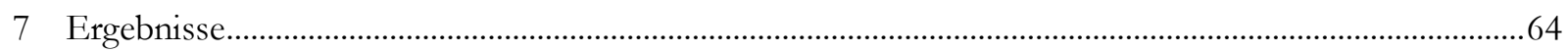

7.1 Rahmendaten zu Schulbegleitung in Stadt und Landkreis Göttingen.........................................65

7.1.1 Person und Qualifikation.................................................................................................6 65

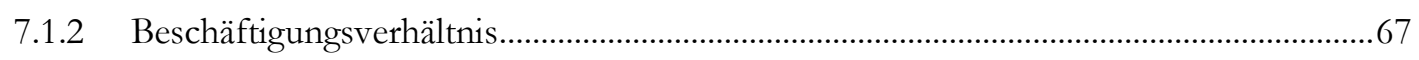

7.1.3 Begleitete Schüler/innen.......................................................................................69

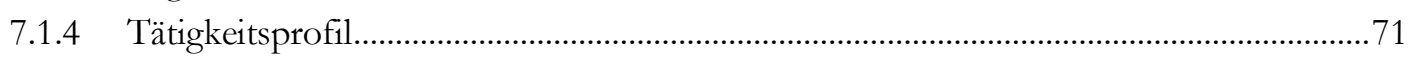

7.1.5 Zusammenhangsanalysen ..................................................................................... 
7.2 Einbindung und Kooperation ............................................................................................

7.2.1 Häufigkeit und Bewertung von Austausch .....................................................................75

7.2.2 Kooperationspraxis mit der Klassenleitung und Kooperationswirkungen. .....................77

7.2.3 Einbindung in die Schulgemeinschaft und Gesamtzufriedenheit ....................................79

7.2.4 Zusammenhänge von Kooperation mit weiteren Merkmalen.............................................80

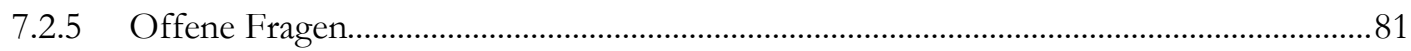

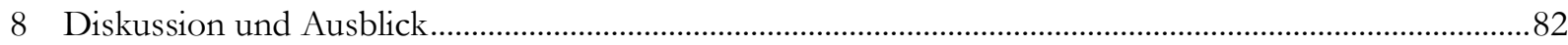

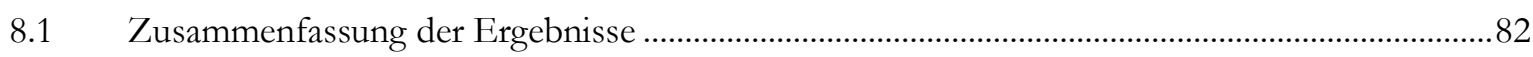

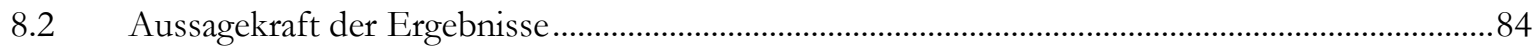

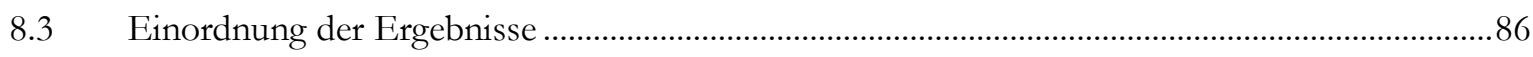

8.4 Implikationen für weitere Forschung und für die schulische Praxis ..............................................87

8.5 Implikationen für die Maßnahme Schulbegleitung .......................................................................8

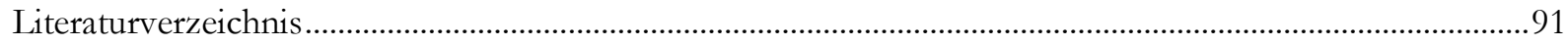

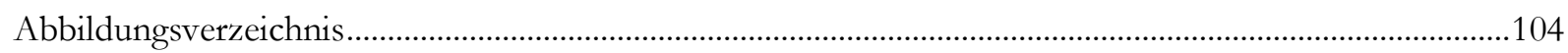

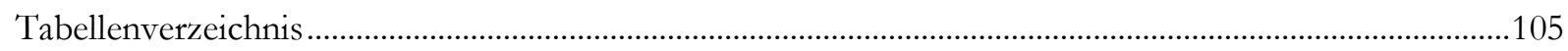

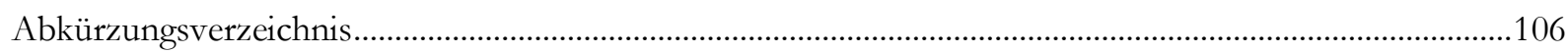




\section{Einleitung und Zielbestimmung}

Schulen gelten traditionell als Orte, an denen wenig professionelle Kooperation ${ }^{1}$ und noch weniger multiprofessionelle Kooperation stattfindet (Lortie, 1975; Terhart, 1999; Weick, 1976); Lehrkräfte werden oft als „Einzelkämpfer“ (Richter \& Pant, 2016, S. 6) gesehen. In den letzten Jahrzehnten hat im deutschen Schulsystem ein weitreichender Veränderungsprozess eingesetzt, dessen Ende noch nicht in Sicht ist und der einen verstärkten Bedarf an Kooperation innerhalb der Lehrerschaft sowie mit anderen Berufsgruppen mit sich bringt (Bauer, 2008; Baumert et al., 2013; Lütje-Klose, 2011).

Ein zentraler Auslöser für die Reformen war der sogenannte PISA2_Schock, als sich zeigte, dass die deutschen Schüler/innen ${ }^{3}$ im internationalen Vergleich schlechter abschnitten als erwartet und ihre Leistungen zudem stark streuten (KMK, 2002). Mit dem Ziel einer Modernisierung des deutschen Schulsystems wurden in der Folge umfassende Maßnahmen beschlossen. Dazu gehörten z. B. der Ausbau von Ganztagsschulen, mit dem sich Erwartungen an eine bessere Förderung insbesondere leistungsschwächerer und bildungsbenachteiligter Schüler/innen verbanden, und die Entwicklung von Bildungsstandards und Schulevaluationen (ebd.). Insgesamt lässt sich eine Entwicklung hin zur Stärkung der Autonomie der Einzelschule feststellen, die sich mit dem Schlagwort Schulentwicklung umschreiben lässt (Bauer, 2008). Einen Wandel im Schulsystem bedeutet auch die Ausweitung inklusiver Beschulung. Vom Deutschen Bildungsrat schon 1973 empfohlen (siehe z. B. Schneider, 2004), hat insbesondere die Ratifizierung der UN-Behindertenrechtskonvention im Jahr 2009 zu einer beschleunigten Umsetzung inklusiver Beschulung beigetragen, die inzwischen in entsprechend geänderten Schulgesetzen Ausdruck findet. Die beschriebenen Entwicklungen führen im Zuge von Schulentwicklungsmaßnahmen (etwa der Entwicklung von Schulprogrammen und der Mitarbeit in Steuergruppen, Bauer, 2008, siehe auch Rolff, 2010) zu verstärkter Kooperation innerhalb des Lehrerkollegiums und im Rahmen von Ganztagsschulen zur Zusammenarbeit mit außerschulischen Partner/innen (Stichwort Netzwerkbildung) und einzelnen Vertreter/innen anderer Berufsgruppen (z. B. Erzieher/innen, Sportübungsleiter/innen). Zur Umsetzung inklusiver Beschulung werden Förderzentren bzw.

1 Kooperation in der Schule bezieht sich in dieser Arbeit durchgängig auf professionelle Kooperation und nicht auf Kooperation mit den Eltern, mit den Schüler/innen oder von Schüler/innen untereinander (etwa beim kooperativen Lernen). Die Arbeit fokussiert zudem auf Lehrkräfte an Regelschulen und auf Kooperation an Regelschulen, an denen die Anwesenheit anderer Professionen im Vergleich zu Förderschulen, die neben Sonderpädagog/innen oftmals auch Therapeut/innen und Pfleger/innen beschäftigen, keine längere Tradition hat.

2 Eine Übersicht über im Text und in den Literaturangaben verwendete einschlägige Abkürzungen findet sich im Abkürzungsverzeichnis.

3 Um die doppelte Nennung einer Personengruppe zu vermeiden, wird auf eine getrennte Nennung der weiblichen und der männlichen Form (Schülerinnen und Schüler) weitgehend verzichtet und stattdessen die Schrägstrich-Variante gewählt (Schüler/innen). Diese Form schließt weibliche und männliche Personen ebenso wie diejenigen, die sich einer anderen oder keiner Kategorie zuordnen, ein. Zur Wahrung der Lesbarkeit wird bei zusammengesetzten Wörtern (Lehrerkooperation) nur die maskuline Form verwendet, die dann generisch zu verstehen ist. 
sonderpädagogische Lehrkräfte, die dafür einen Teil ihrer Arbeitszeit an Regelschulen verbringen (Boban \& Hinz, 2008), einbezogen. Dass Kooperation inzwischen als Teil des Lehrerberufs gesehen wird, zeigen die geänderten Standards für Lehrerbildung der Kultusministerkonferenz (KMK, 2004; siehe auch Blömeke, Reinhold, Tulodziecki \& Wildt, 2004; Frey, 2014).

Im wissenschaftlichen Fachdiskurs wird schulischer Kooperation hohe Bedeutung beigemessen. Im Schulqualitätsmodell von Ditton (2000) wird Kooperation als Qualitätsmerkmal von Schule gesehen, im Schulentwicklungsmodell von Rolff (2010) als wichtiges Element in der Organisationsentwicklung. Von Kooperation an Schulen werden Entlastung für die einzelnen Lehrkräfte, eine höhere Schuleffektivität und innovative Impulse für die Schulentwicklung und für die Professionalisierung der Lehrkräfte erwartet (Bauer, 2008; Fussangel, 2008; Kuper \& Kapelle, 2012; Steinert et al., 2006). Theoretisch diskutiert und empirisch untersucht werden diese Annahmen vor allem in Bezug auf die Kooperation von (Regel-)Lehrkräften untereinander, wobei im Zuge der Ganztagsschulforschung auch verstärkt multiprofessionelle Kooperation in den Blick gerät (z. B. Arnoldt, 2008a, 2008b; Breuer, 2011, 2015; Dizinger, 2015; Dizinger \& Böhm-Kasper, 2012; Dizinger, Fussangel \& Böhm-Kasper, 2011 ; Drossel \& Willems, 2014; LehmannWermser, Naacke, Nonte \& Ritter, 2010; Rahm, Rabenstein \& Nerowski, 2015; Reh \& Breuer, 2012; Speck, Olk \& Stimpel, 2011a, 2011b; Steiner, 2013; Steiner \& Tillmann, 2011; Tillmann \& Willems, 2016). Kooperation in inklusiven Settings wird meist separat thematisiert, oft im sonder- bzw. heilpädagogischen Fachdiskurs, und bezieht sich vornehmlich auf die Kooperation zwischen Regellehrkräften und sonderpädagogischen Lehrkräften. Dabei wird Kooperation einhellig als zentraler Gelingensfaktor für die gemeinsame Beschulung von Schüler/innen mit und ohne Behinderung gesehen (z. B. Baumert et al., 2013; Boban \& Hinz, 2003; Böing, 2013; Lelgemann, Singer, Walter-Klose \& Lübbeke, 2012; Lütje-Klose, 2011; Mays, 2014; Moser \& Demmer-Dieckmann, 2013) und nimmt z. B. im Index für Inklusion (Index for inclusion), einem international rezipierten Leitfaden zur inklusiven Schulentwicklung, eine wichtige Rolle ein (Boban \& Hinz, 2003 bzw. Booth \& Ainscow, 2002). Im Zusammenhang mit der Zusammenarbeit von Regellehrkräften und sonderpädagogischen Lehrkräften werden vor allem Herausforderungen der multiprofessionellen Zusammenarbeit thematisiert, wobei Aspekte der Teilung von Aufgaben und Zuständigkeiten sowie der Rollenklärung im Vordergrund stehen (z. B. Brähler, 1991; Gebhard et al., 2014; Kron, 2012; Lütje-Klose \& Willenbring, 1999; Moser, 2013; Wessel, 2005).

Relativ unbeachtet vom Kooperationsdiskurs und von der Kooperationsforschung in der Schul- und Sonderpädagogik ist eine Akteursgruppe, die trotz kontroverser Diskussionen um die Angemessenheit ihres Einsatzes (z. B. AGJ, 2014; Bloemer-Hausmanns, 2014; Böing, 2013; Dworschak, 2012a; Fischer, 2011; Keil, Baier, Friedemann \& Stange, 2010; Knuf, 2013; Köpfer, 2013a, 2013b; Lindmeier \& Polleschner, 2014; Lübeck, 2016b; Schöler, 2002) an vielen Schulen inzwischen fester Bestandteil ist: Schulbegleiter/innen. ${ }^{4}$ Laut dem Niedersächsischen Landesinstitut für schulische Qualitätsentwicklung ist darunter „eine meist langfristig eingesetzte Eingliederungshilfe“ für Schüler/innen, „die aufgrund ihrer Behinderung auf individuelle Unterstützung angewiesen sind“ (NLQ, 2016), zu verstehen. Die Maßnahme Schulbegleitung wird vom Jugend- oder Sozialamt bewilligt und in der Regel von externen Trägern durchgeführt. Schulbegleiter/innen sind kein schuleigenes Personal und arbeiten grundsätzlich einzelfallbezogen. Ihr Aufgabenbereich ist auf den individuellen Bedarf des begleiteten Kindes oder Jugendlichen abgestimmt und kann ,z. B. Pflegehilfe, Hilfestellung im Unterricht, Unterstützung im sozialen und emotionalen Bereich und der Kommunikation" umfassen (ebd.). Die Zahl an Schulbegleitungen ist in den letzten zwei Jahrzehnten sprunghaft gestiegen (Beck, Dworschak \& Eibner, 2010; Dworschak, 2012b; Kißgen, Franke, Ladinig, Mays \& Carlitscheck, 2013; Lindmeier, Polleschner \& Thiel, 2014). Zurückführen lässt sich diese Entwicklung unter anderem auf veränderte rechtliche Rahmenbedingungen zur schulischen Inklusion und, zusammenhängend damit, auf die Ausweitung inklusiver Beschulung (siehe hierzu z. B. Lindmeier \& Polleschner, 2014).

Trotz der gestiegenen Fallzahlen und obwohl die Bemühungen um Inklusion im Schulsystem einen weiteren Anstieg vermuten lassen, existiert nach wie vor kein einheitliches Konzept für Schulbegleitung, vielmehr ist „die Ausformung dieser Tätigkeit [...] historisch und regional gewachsen“ (Knuf, 2013, S. 93). Es ist weitgehend dem bewilligenden Amt überlassen die Aufgaben und Ziele der jeweiligen Maßnahme festzulegen, wobei die Durchführung in der Regel durch freie Träger übernommen und begleitet wird (Dworschak, 2010; Greß, 2014). Da die Schulbegleitung überwiegend im Unterricht stattfindet, kommt der Kooperation zwischen den Lehrkräften und der Schulbegleitung eine zentrale Bedeutung zu, insbesondere

4 In der Fachliteratur hat sich der Begriff Schulbegleitung durchgesetzt, synonym verwendet werden aber auch die Begriffe Integrationshilfe, Unterrichtsassistenz und Integrationsassistenz. 
der Klassenleitung des begleiteten Kindes oder Jugendlichen, die verantwortlich bleibt für dessen schulische Entwicklung. Eine Herausforderung ist dabei die Definition des Zuständigkeitsbereichs von Schulbegleitung. Gesetzlich ist eine strikte Trennung zwischen Aufgaben, die Schule leisten muss, und Aufgaben der Eingliederungshilfe vorgeschrieben. Hinzu kommt, dass Schulbegleitung keine Qualifikation voraussetzt und ein Einsatz über das vorgesehene Aufgabenfeld hinaus Legitimationsprobleme aufwerfen würde. Unter anderem hieraus speist sich die kontroverse Diskussion, die um den Einsatz von Schulbegleiter/innen geführt wird (z. B. AGJ, 2014; Dworschak, 2012c; Kißgen et al., 2013; Lindmeier \& Polleschner, 2014). Das Thema Schulbegleitung findet häufig Erwähnung in allgemeinen Ratgeberbüchern oder Erfahrungsberichten über die integrative bzw. inklusive Beschulung ${ }^{5}$ von Kindern oder Jugendlichen mit Behinderung an Regelschulen (z. B. Conée \& Ranscht, 2012; Götzinger, Richter, Scheidle, Scheidle \& Wittmann, 2012; Lautenschläger, 2014; Loos, 2014; Niedermayer, 2009; Schuster, 2013). Die fachwissenschaftlichen Publikationen zum Thema sind zahlenmäßig bislang noch recht überschaubar, vor allem im empirischen Bereich. Die vorhandenen Studien liefern meist einen Überblick über lokale Rahmendaten zu Schulbegleitung, etwa zum Qualifikationshintergrund der Schulbegleiter/innen, zu den Schulformen, den Beantragungsgründen und der Entwicklung der Fallzahlen (z. B. Henn et al., 2014; Keil et al., 2010; Kißgen et al., 2013). Kooperationsspezifische Daten wurden quantitativ nur vereinzelt und wenn, dann vorwiegend in allgemeiner Form erfasst, indem nach der globalen Zufriedenheit mit der Zusammenarbeit mit der Klassenleitung oder den Eltern gefragt wurde (Wohlgemuth, 2009; Zauner \& Zwosta, 2014). Auch in der qualitativen Forschung ist das Thema Kooperation bislang nur vereinzelt oder am Rande analysiert worden (Deger, Puhr \& Jerg, 2015; Lindmeier et al., 2014; Lübeck \& Heinrich, 2016). Insbesondere fehlt es an quantitativen Studien, die die Schulbegleitungspraxis aufbauend auf theoretischen Modellen systematisch untersuchen.

Mit dieser Arbeit soll diese Forschungslücke ein Stück weit geschlossen werden, indem die schulische Kooperation mit Schulbegleiter/innen untersucht wird. Aufgrund der wenigen vorliegenden Daten zu Schulbegleitung allgemein und zur Frage der Kooperation im Speziellen ist die Untersuchung weitgehend deskriptiv bzw. explorativ angelegt. Ziel ist zum einen, die vorhandenen lokalbezogenen Daten zur Beschreibung von Schulbegleiter/innen und Schulbegleitung um Daten aus Stadt und Landkreis Göttingen zu ergänzen und zum anderen Einblicke zu gewinnen, inwieweit und in welcher Form Schulbegleiter/innen in schulische Strukturen eingebunden sind und wie sich die Zusammenarbeit mit verschiedenen Personengruppen gestaltet. Im Fokus steht dabei die Kooperation mit der Klassenleitung ${ }^{6}$, die als wichtigste Kooperationspartnerin angenommen wird. Aus Kapazitätsgründen wird im Rahmen der als Fragebogenstudie angelegten Untersuchung nur die Perspektive der Schulbegleiter/innen erfasst. Die Kooperation zwischen Klassenleitung und Schulbegleitung wird als multiprofessionelle Kooperation verstanden. Obwohl multiprofessionelle Kooperation in verschiedenen Studien qualitativ und, insbesondere in der StEG-Studie, auch quantitativ untersucht wurde (z. B. Arnoldt, 2008a, 2008b; Steiner \& Tillmann, 2011), fehlt es bislang an ausgearbeiteten Ansätzen und Modellen, wie sie für die Lehrerkooperation bereits vorliegen (vgl. Boller, 2012). Da davon ausgegangen werden kann, dass für die Kooperation von Lehrkräften und Schulbegleiter/innen die Rahmenbedingungen, die Schule setzt, prägend sind (vgl. Dizinger, 2015), wird dennoch dem theoretischen Anschluss an die Schulforschung der Vorrang gegeben gegenüber dem Anschluss an Kooperationsmodelle aus anderen Wissenschaftsdisziplinen (vgl. Boller, 2012). Die Zusammenarbeit von Lehrkräften und Schulbegleiter/innen wird in Anlehnung an das Lehrerkooperationsmodell von Gräsel, Fussangel und Pröbstel (2006) bzw. Fussangel (2008) konzeptualisiert, das bereits in verschiedenen Studien zur schulischen Kooperationsforschung (Dizinger, 2015; Dizinger et al., 2011a, 2011b; Richter \& Pant, 2016) in originaler oder angepasster Form angewandt wurde. Das Modell unterscheidet zwischen verschiedenen Formen von Kooperation, die in unterschiedlicher Stärke mit erwarteten Wirkungen von Kooperation zusammenhängen (Fussangel, 2008). Die Entwicklung von Hypothesen zur Wirkung von verschiedenen Kooperationsformen zwischen Klassenleitung und Schulbegleitung erfolgt zum einen mit Rekurs auf fachwissenschaftliche Analysen zu Lehrerkooperation und multiprofessioneller Kooperation. Zu beidem, insbesondere zu Lehrerkooperation, kann dabei auf umfangreiche empirisch gewonnene Erkenntnisse zurückgegriffen werden (für einen Überblick siehe Böttcher, Maykus, Altermann \& Liesegang, 2011; Fussangel \& Gräsel, 2014). Zum anderen bieten die vorhandenen Informationen zu Schulbegleitung die Basis für die

5 In dieser Arbeit werden alle aktuellen Formen gemeinsamer Beschulung von Schüler/innen mit und ohne Behinderung als inklusive Beschulung bezeichnet. Auf Unterrichtsebene wird von inklusivem Unterricht (im Kontext der Zusammenarbeit zwischen Schul- und Sonderpädagogik auch von Gemeinsamem Unterricht) gesprochen.

6 Die Bezeichnung schließt auch Klassenleitungsteams ein. 
Entwicklung allgemeiner Forschungsfragen und spezieller Hypothesen zur Kooperation mit der Klassenleitung. Da bislang wenig empirische Schulbegleitungsforschung insbesondere zu der Kooperationsfrage vorliegt, bilden nichtempirische Arbeiten und allgemeine Informationstexte zum Thema eine wichtige Informationsquelle, die ergänzt wird durch mehrere Gespräche, die ich im Vorfeld der Studie mit Vertreter/innen der zuständigen Ämter, der Träger von Schulbegleitung und einer Schulbegleiterin geführt habe. Zusätzlich werden zentrale Erkenntnisse aus der internationalen Forschung zum Einsatz von Assistenzpersonal im Unterricht, das in mehrerlei Hinsicht Parallelen zu Schulbegleitung aufweist, einbezogen.

Die Anlage sowohl der Studie als auch dieser Arbeit ist insofern zweiteilig, als dass sie sich dem Thema Kooperation zwischen Schulbegleitung und Lehrkräften zum einen aus der Perspektive von Schulbegleitung nähert und zur Gewinnung empirischer Daten in diesem noch wenig bearbeiteten Forschungsfeld beitragen soll. Zum anderen ist die Untersuchung theoretisch eingebettet in die stärker lehrerbezogene schulische Kooperationsforschung. Auch wenn der Rahmen dieser Arbeit keine parallele Befragung von Schulbegleiter/innen und Lehrkräften zulässt, hat die Untersuchung auch zum Ziel, Erkenntnisse für die Ausbildung und die schulische Praxis von Lehrkräften zu gewinnen, die zunehmend vor der Aufgabe stehen, mit anderen Professionen zu kooperieren oder, wie Steiner und Tillmann (2011) es ausdrücken, vor der dringenden Notwendigkeit der Entwicklung „,interprofessionelle[r] Kompetenz“ (S. 51). Auch wenn Schulbegleiter/innen kein schuleigenes Personal darstellen, sind Ansatzpunkte für die Übertragbarkeit auf andere schulische Kooperationspartner/innen zu erwarten. Wie das Personal, das an Ganztagsschulen mitwirkt, arbeiten Schulbegleiter/innen nicht als Lehrkräfte, und wie die sonderpädagogischen Lehrkräfte arbeiten sie mit den Regellehrkräften schülerbezogen im Unterricht zusammen.

Die Arbeit gliedert sich in einen theoretischen und einen empirischen Teil. Der theoretische Teil besteht aus einem Kapitel zu Schulbegleitung und einem Kapitel zu Kooperation an Schulen. Beide Themen werden darin zunächst theoretisch erörtert, daraufhin wird der Forschungsstand aufgearbeitet und am Ende steht jeweils eine kurze Zusammenfassung. Zum Verständnis der Maßnahme Schulbegleitung kommen rechtliche Grundlagen zur Sprache und es wird die lokale Umsetzung der Maßnahme in Stadt und Landkreis Göttingen erläutert, die sowohl der beispielhaften Darstellung der Bewilligungs- und Durchführungspraxis dient als auch den regionalspezifischen Hintergrund der Schulbegleiter/innen aufzeigt, aus denen die Stichprobe gewonnen wurde. Die lokale Bedeutsamkeit des Themas Schulbegleitung wird daran deutlich, dass auch hier ein sprunghafter Anstieg der Fallzahlen verzeichnet wird. Der daraufhin dargestellte nationale Forschungsstand zu Schulbegleitung wird zuletzt ergänzt durch den Bezug auf internationale Forschung zu Assistenzpersonal im inklusiven Unterricht. Das Kapitel zu Kooperation nähert sich dem Thema Kooperation in Schulen zunächst theoretisch durch die Begriffsbestimmung von Kooperation und die Darstellung der für Schule charakteristischen Rahmenbedingungen für Kooperation und ihrer Veränderungen in jüngster Zeit. Anschließend wird die Bedeutung, die Kooperation für Innovations- und Professionalisierungsprozesse zugeschrieben wird, diskutiert und abschließend werden Besonderheiten multiprofessioneller Kooperation erarbeitet und Modelle schulischer Kooperation vorgestellt. Die Darstellung des darauf folgenden Forschungsstandes gliedert sich in die Darstellung von Befunden zu Lehrerkooperation und zu multiprofessioneller Kooperation. Zur Überleitung in den empirischen Teil der Arbeit werden im Anschluss zentrale Erkenntnisse aus den Kapiteln zu Schulbegleitung und zu Kooperation zusammengefasst und daraus Perspektiven für die eigene Arbeit abgeleitet. An dieser Stelle erfolgt auch die Adaption des Lehrerkooperationsmodells von Fussangel (2008). Im empirischen Teil werden zunächst die aus dem theoretischen Teil abgeleiteten allgemeinen Forschungsfragen und speziellen Hypothesen vorgestellt, bevor die Darstellung der empirischen Untersuchung einschließlich der Entwicklung des Fragebogens erfolgt. An die Ergebnisdarstellung schließt sich in der Diskussion die Interpretation der Befunde und ihre Einordnung in bisherige Forschung an. Dabei werden zum einen Implikationen für die weitere Forschung zu Schulbegleitung und schulischer Kooperation benannt sowie die Nutzbarkeit des entwickelten Messinstruments für weitere Untersuchungen diskutiert. Zum anderen wird nach der Bedeutsamkeit der Ergebnisse für die Praxis gefragt, indem versucht wird, sowohl Schlussfolgerungen für die Weiterentwicklung der Maßnahme Schulbegleitung als auch für die Lehrerbildung und die Weiterbildung von Lehrkräften abzuleiten. 


\section{Schulbegleitung}

Im Folgenden soll ein systematischer Überblick über die Maßnahme Schulbegleitung und den aktuellen Forschungsstand gegeben werden. Hierzu erfolgt zunächst eine Erläuterung der rechtlichen Grundlagen von Schulbegleitung. Da sich die konkrete Ausgestaltung von Schulbegleitung regional unterscheidet, befasst sich das darauffolgende Teilkapitel mit der Umsetzung der Maßnahme in Stadt und Landkreis Göttingen, aus dem sich die Stichprobe der Untersuchung zusammensetzt. Der Skizzierung zentraler Diskussionspunkte um Schulbegleitung folgt schließlich die Darstellung empirischer Befunde. Obwohl Schulbegleiter/innen vielerorts bereits fester Bestandteil des Schullebens sind und gleichzeitig die grundlegende Konzeption und Organisation dieser Maßnahme in vielen Punkten strittig ist, hat sich die empirische Forschung des Themas bislang nur vereinzelt angenommen. Die vorhandenen Studien erheben ausnahmslos Querschnittsdaten. Meist stehen dabei Fragen nach der Anzahl und dem Umfang der Inanspruchnahme an verschiedenen Schulformen sowie die Qualifikationshintergründe und Tätigkeitsfelder der Schulbegleiter/innen im Vordergrund. Abfragen zur kooperativen Ausgestaltung der Maßnahme und zu ihrer Bewertung werden, wenn überhaupt, nur in allgemeiner Form gemacht. Aufgrund der Forschungslücken zu Schulbegleitung wird der Forschungsstand hierzu erweitert um eine Perspektive auf internationale Forschung zum Einsatz von Assistenzkräften in der inklusiven Beschulung. Parallelen zu Schulbegleitung werden in der abschließenden Kapitelzusammenfassung diskutiert.

\subsection{Schulbegleitung als Maßnahme der Eingliederungshilfe}

In ihren Anfängen diente Schulbegleitung dazu, Kindern und Jugendlichen mit besonderem Pflegebedarf den Besuch einer (damals: Sonder-)Schule zu ermöglichen und wurde teilweise über die Krankenkassen finanziert (Dworschak, 2010; Knuf, 2013). Inzwischen wird Schulbegleitung fast ausschließlich als Eingliederungshilfe durch die Jugendhilfe bzw. Sozialhilfe geleistet (Henn et al., 2014). Auch in diesem Rahmen ist Schulbegleitung eine einzelfallbezogene Maßnahme mit dem Ziel, schulische Teilhabe für Schüler/innen mit Behinderung zu gewährleisten. Eingliederungshilfe im Sinne der Sozialgesetzgebung beschränkt sich aber nicht nur auf die Ermöglichung des Unterrichtsbesuchs, sondern auch auf die soziale Teilhabe innerhalb der Klassen- und Schulgemeinschaft (Schwarz, 2012). Zum Verständnis der spezifischen Charakteristika und aktueller Kontroversen um die Maßnahme Schulbegleitung ist eine Erläuterung des rechtlichen Hintergrunds unerlässlich. Im Anschluss an die Darstellung der rechtlichen Grundlagen erfolgt eine Übersicht über den Ablauf der Bewilligung und die Ausgestaltung der Maßnahme Schulbegleitung in Stadt und Landkreis Göttingen. Diese Darstellung dient zum einen als lokales Beispiel für die Umsetzung der allgemeinen Rahmenbedingungen und beleuchtet zum anderen die Ausgestaltung der Maßnahme in dem Gebiet, 
in dem die befragten Schulbegleiter/innen tätig sind. Die Informationen in diesem Teil entstammen Gesprächen, die im Vorfeld der Untersuchung mit Zuständigen der Ämter, den Trägern für Schulbegleitung und der Fachstelle für Diagnostik geführt wurden. Zuletzt werden aktuelle Diskussionsstränge zu Schulbegleitung als Unterstützungsmaßnahme für Schüler/innen mit Behinderung nachgezeichnet.

\subsubsection{Rechtliche Grundlagen}

Die Voraussetzungen zur Bewilligung von Schulbegleitung sind in den Sozialgesetzbüchern festgelegt. Ob die Kriterien zur Bewilligung erfüllt sind, hängt im Einzelnen aber nicht nur von den Vorgaben der Sozialgesetzbücher ab, sondern auch von weiteren rechtlichen Rahmenbedingungen zur inklusiven Beschulung, von denen die wichtigsten in der UN-Behindertenrechtskonvention und in den Schulgesetzen der Länder geregelt sind. Die Darstellung der schulgesetzlichen Rahmenbedingungen beschränkt sich an dieser Stelle auf Niedersachsen. Beginnend mit den rechtlichen Grundlagen inklusiver Beschulung werden im zweiten Schritt die Vorgaben der Sozialgesetzbücher erläutert, nach denen Schulbegleitung als eine Maßnahme der Eingliederungshilfe für Menschen mit Behinderung geleistet wird.

Inklusive Beschulung. In Deutschland hat das Sonderschulwesen eine lange Tradition (Bundschuh \& Baier, 2009). Zwar gibt es Modellversuche integrativen Lernens, meist auf Initiative von Eltern, schon seit den 1950er Jahren (Brähler, 1991) und bereits 1973 empfahl der Bildungsrat die gemeinsame Beschulung von Kindern mit und ohne Behinderung (Brähler, 1991; Eberwein, 1998). Zahlenmäßige Bedeutsamkeit hat diese Form der Beschulung aber erst in den letzten zwei Jahrzehnten erreicht und der überwiegende Teil von Schüler/innen mit sonderpädagogischem Förderbedarf wird nach wie vor in speziellen Förderschulen unterrichtet (Autorengruppe Bildungsberichterstattung, 2014). Einen Meilenstein in der Entwicklung hin zur inklusiven Schule bildet die UN-Behindertenrechtskonvention, die 2009 in bundesdeutsches Recht überführt wurde. Artikel 24 legt die Gewährleistung eines ,integrative[n] Bildungssystem[s] auf allen Ebenen“ fest und verpflichtet die Vertragsstaaten, dass sie „,wirksame individuell angepasste Unterstützungsmaßnahmen in einem Umfeld, das die bestmögliche schulische und soziale Entwicklung gestattet", sicherstellen. In Anlehnung an die Behindertenrechtskonvention verabschiedete die Kultusministerkonferenz (2011) die Empfehlungen Inklusive Bildung von Kindern und Jugendlichen mit Bebinderungen in Schulen. Unter anderem werden darin der Nachteilsausgleich ${ }^{7}$ und die sonderpädagogische Förderung an Allgemeinen Schulen als Unterstützungsmaßnahmen aufgeführt. ${ }^{8}$ Es bleibt den Ländern überlassen, wie sie diese Maßnahmen der individuellen Förderplanung (Knaack, 2006) in ihren Schulgesetzen ausgestalten und wie sie den Inklusionsprozess im Detail umsetzen, an dessen Ende die eine Schule für alle stehen soll.

Im Niedersächsischen Schulgesetz (NSchG) ist bereits in der Fassung von 1998 in $₫ 4$ von inklusiver Schule die Rede. Im Jahr 2012 wurden mit dem Gesetz der inklusiven Schule Änderungen im Schulgesetz vorgenommen, die konkrete Maßnahmen zur Umsetzung von Inklusion im Bildungssystem vorschreiben. Festgelegt sind der Nachteilsausgleich und die Sicherstellung sonderpädagogischer Unterstützung an Allgemeinen Schulen, zudem sehen die Änderungen den Abbau der Förderschulen und ein gestärktes Elternwahlrecht auf inklusive Beschulung vor. Seit dem Schuljahr 2013/14 laufen die Förderschulen mit dem Förderschwerpunkt Lernen aus und aktuell (in der Fassung des NSchG von 2015) ist vorgesehen, dass für den Förderschwerpunkt Sprache keine Neugründungen von Förderschulen mehr möglich sind. Für alle anderen Förderschwerpunkte (Emotionale und soriale Entwicklung, Geistige Entwicklung, Körperliche und motorische Entwicklung, Hören, Sehen $)^{9}$ gilt, dass die Entscheidung für eine Förder- oder Allgemeine Schule den Erziehungsberechtigten obliegt. Allgemeine Schulen sind damit verpflichtet, auch Schüler/innen mit Bedarf an sonderpädagogischer Unterstützung aufzunehmen, wobei bis 2024 die Möglichkeit besteht, regionale Schwerpunktschulen zu bestimmen, denen ein/e Schüler/in je nach Art des Unterstützungsbedarfs zugewiesen

So könnten Schulen einen individuellen Nachteilsausgleich gewähren, etwa indem die mündliche Note eines Schülers oder einer Schülerin mit Autismusdiagnose weniger gewichtet wird oder eine Zeit lang ganz auf Zensurengebung verzichtet wird (vgl. Knaack, 2006).

8 Schulbegleitung als Maßnahme findet keine Erwähnung in den Empfehlungen der Kultusministerkonferenz, es wird aber zumindest auf die Bedeutsamkeit der Zusammenarbeit mit Assistenzpersonal hingewiesen und Träger der Eingliederungshilfe als außerschulische Kooperationspartner/innen erwähnt.

9 Die Kultusministerkonferenz nennt (langandauernde) Erkrankung als weiteren Förderschwerpunkt (Autorengruppe Bildungsberichterstattung, 2014; siehe hierzu KMK, 1999), der in der Fachliteratur aber nur selten Erwähnung findet. Das NSchG sieht keinen entsprechenden Förderschwerpunkt vor und legt lediglich fest, dass längerfristig erkrankten Schüler/innen „Unterricht zu Hause oder im Krankenhaus in angemessenem Umfang“" erteilt werden soll (\$69 Abs. 1). 
werden kann. Ausgenommen von der Schwerpunktregelung sind Grundschulen hinsichtlich der Förderschwerpunkte Lernen, Sprache und Emotionale und soziale Entwicklung. Grundschulen erhalten eine generell sonderpädagogische Grundversorgung in Form von Förderschullehrerstunden, weiterführende Schulen erhalten diese Unterstützung nach Bedarf. Für sonderpädagogische Hilfen, die personell nicht fest an einer Schule verankert sind, hat sich der Begriff Mobiler Dienst etabliert.

Die rechtlichen Grundlagen für die Bewilligung einer Schulbegleitung bleiben von Schulgesetzänderungen zwar unberührt, dennoch ist davon auszugehen, dass die Änderungen im Niedersächsischen Schulgesetz die Zahl an Schulbegleitungen steigen lassen. Das gestärkte Elternwahlrecht sowie der Abbau der Förderschule mit dem Förderschwerpunkt Lernen führt dazu, dass einerseits der Ablehnungsgrund, an einer Förderschule sei keine Begleitung nötig (Dworschak, 2010; Prieß, 2009; Schwarz, 2012), kaum noch haltbar ist und andererseits aufgrund steigender Zahlen inklusiver Beschulung von Schüler/innen mit Bedarf an sonderpädagogischer Unterstützung der Bedarf bzw. die Beantragungshäufigkeit von Schulbegleitung steigen werden (siehe hierzu Schnell, 2014).

Schulbegleitung im Rahmen von Jugend- und Sozialhilfe. Eingliederungshilfe ist eine in den Sozialgesetzbüchern verankerte Unterstützungsleistung für Menschen mit Behinderung. Grundlegend für die Einstufung als behindert im Sinne des Sozialrechts sind folgende Voraussetzungen:

Menschen sind behindert, wenn ibre körperliche Funktion, geistige Fähigkeit oder seelische Gesundheit mit hoher Wabrscheinlichkeit länger als sechs Monate von dem für das Lebensalter typischen Zustand abweichen und daber ibre Teilhabe am Leben in der Gesellschaft beeinträchtigt ist. Sie sind von Bebinderung bedrobt, wenn die Beeinträcbtigung zu erwarten ist. ( $\$ 2$ Abs. 1 SGB IX)

Mit diesem zweigliedrigen Behinderungsbegriff orientiert sich das Sozialrecht an der Behinderungsdefinition der Weltgesundheitsorganisation WHO, in der neben medizinischen Aspekten auch das Soziale Modell von Behinderung Eingang gefunden hat (Banafsche, 2013), das die Kontextbezogenheit von Beeinträchtigungen berücksichtigt. ${ }^{10}$ Aufgabe der Eingliederungshilfe ist es, die aus einer körperlichen, seelischen oder geistigen Normabweichung drohende oder resultierende Beeinträchtigung der gesellschaftlichen Teilhabe zu mildern oder zu beseitigen ( $\$ 54$ Abs. 3 SGB XII; siehe auch Ernst, 2013). Bildung wird dabei als ein zentraler Bereich gesellschaftlicher Teilhabe gesehen (siehe auch KMK, 2011). Die Voraussetzungen für den Anspruch auf Eingliederungshilfe sind von daher nur dann gegeben, wenn eine entsprechende Normabweichung ${ }^{11}$ und eine Teilhabegefährdung vorliegen - oder drohen einzutreten -, und ersteres ursächlich für letzteres ist (\$53 Abs. 1 SGB XII). Eingliederungshilfe wird deshalb nicht pauschal für bestimmte Menschengruppen geleistet, sondern ist immer einzelfallbezogen. Während bei Kindern und Jugendlichen mit geistigen und körperlichen Behinderungen Eingliederungshilfe nach dem SGB XII (Sozialhilfe) geleistet wird, findet bei Kindern und Jugendlichen mit seelischen Behinderungen das SGB VIII (Kinder- und Jugendhilfe) Anwendung.

Nach dem SGB XII wird Schulbegleitung als eine der „Hilfen zu einer angemessenen Schulbildung“ ( $\$ 54$ Abs. 1 S. 1) gewährt; in $\$ 12$ EinglHVO, der $\$ 54$ näher ausführt, werden diese Hilfen beschrieben als „sonstige Maßnahmen zugunsten körperlich und geistig behinderter Kinder und Jugendlicher, wenn die Maßnahmen erforderlich und geeignet sind, dem behinderten Menschen den Schulbesuch im Rahmen der allgemeinen Schulpflicht zu ermöglichen oder zu erleichtern“. Nach dem SGB VIII wird Schulbegleitung schlicht als „Hilfe in ambulanter Form“ (\$35a Abs. 2 S. 1) geleistet. Da das SGB VIII bezüglich Aufgaben, Zielen und Leistungen der Eingliederungshilfe auf das SGB XII verweist (\$35a Abs. 3), ist die Maßnahme Schulbegleitung in beiden Fällen im Wesentlichen den gleichen Richtlinien unterworfen (siehe auch Greß, 2014). Das duale Modell der getrennten Zuständigkeit je nach Behinderungsart wird schon seit längerer Zeit kritisch diskutiert. ${ }^{12}$ Aktuell plädieren unter anderem Vertreter/innen der Kinder- und Jugendpsychiatrie für

10 Ein anschauliches Beispiel hierfür ist Sehschwäche, die abhängig von der Verfügbarkeit von Sehhilfen eine Behinderung darstellen kann.

11 Im Folgenden wird das, was nach SGB IX als Normabweichung beschrieben ist, weiterhin als Behinderung bezeichnet. Damit weiche ich vom Sprachgebrauch des SGB IX ab, das Behinderung als Zusammentreffen der Normabweichung und der Teilhabebeeinträchtigung auffasst, und verwende den Begriff so, wie er in den meisten Kontexten gebraucht wird, unter anderem auch im SGB VIII und im SGB XII.

12 Ein Grund für die Kritik ist, dass die Bewilligungsschwelle für Eingliederungshilfe nach SGB XII höher angesetzt ist als nach SGB VIII: Das SGB XII setzt die Wesentlichkeit der Beeinträchtigung sowie die Aussicht auf Erfüllbarkeit der Aufgabe der Eingliederungshilfe voraus. Die praktischen Auswirkungen dieser Ungleichheit in den Anspruchsvoraussetzungen sind aber schwer einzuschätzen (vgl. Banafsche, 2013; Fegert \& Schepker, 2014). 
die „Große Lösung“, nämlich dafür, Leistungen für Kinder und Jugendliche mit Behinderung generell über die Kinder- und Jugendhilfe zu gewähren (Fegert \& Schepker, 2014).

Verantwortlich für die Antragstellung beim Jugend- oder Sozialamt sind die Erziehungsberechtigten; liegt eine Mehrfachbehinderung vor, ist in der Regel das Sozialamt zuständig. Um die Anspruchsvoraussetzungen zu prüfen, holt das jeweilige Amt ein Fachgutachten über die behinderungsrelevante Diagnose des Kindes oder Jugendlichen ein. Es ist den Eltern möglich, bereits vorliegende oder eigens eingeholte Gutachten vorzulegen, die Ämter dürfen einen amtsärztlichen Gutachter aber prinzipiell selbst bestimmen (Banafsche, 2013). Dieser medizinischen bzw. psychologischen oder psychiatrischen Diagnose folgt ggf. die Prüfung der vorliegenden oder drohenden Teilhabegefährdung durch das Amt selbst. Sind die Voraussetzungen für Schulbegleitung erfüllt, werden im Rahmen der individuellen Hilfeplanung (Knaack, 2006) der Umfang, die zeitliche Befristung und die Gestaltung der Maßnahme festgelegt. An die Eltern ergeht ein Bewilligungsbescheid mit den entsprechenden Informationen. Mit der Ausführung betrauen die Ämter als Kostenträger in der Regel einen freien Träger der Jugend- bzw. Sozialhilfe (Dworschak, 2010; Greß, 2014). Bis auf Ausnahmen übernehmen die Ämter nur Kosten auf der Stundensatzberechnung eines Helfergehalts, qualifizierte Kräfte sind in der Regel nicht vorgesehen (Dworschak, 2010). Alternativ können die Antragsteller auf Wunsch die bewilligte Eingliederungshilfe in Form eines Persönlichen Budgets erhalten ( $\$ 57$ SGB XII), d. h. sie erhalten Geld anstelle einer Sachleistung und treten selbst als Arbeitgeber auf. Dieser Fall scheint eher selten vorzukommen (Ernst, 2013). Nach Ablauf der Maßnahme wird sie nur auf erneute Antragstellung hin ggf. verlängert.

\subsubsection{Bewilligungs- und Durchführungspraxis in Stadt und Landkreis Göttingen}

Das Gebiet Stadt und Landkreis Göttingen umfasst vier Ämter, die für Schulbegleitungsanträge zuständig sind: Das Jugendamt der Stadt Göttingen, das Jugendamt des Landkreises Göttingen, das Sozialamt der Stadt Göttingen und das Sozialamt des Landkreises Göttingen. Der Wohnort des Kindes oder Jugendlichen entscheidet, welches Amt zuständig ist. Jedes der vier Ämter arbeitet in der Sache unabhängig von den anderen. Bei den Sozialämtern erfolgt medizinische Prüfung durch das Gesundheitsamt, das auch bei der Teilhabeprüfung mitwirkt. Die Entscheidung treffen letztlich die Mitarbeiter/innen des Amts. Die Jugendämter (die im Übrigen für den Bereich Schulbegleitung Koordinatorenstellen eingerichtet haben) lassen sowohl die psychologisch-psychiatrische Diagnostik als auch die Teilhabeprüfung durch die Fachstelle für Diagnostik durchführen. ${ }^{13}$

Die Fachstelle für Diagnostik befindet sich in Trägerschaft der Jugendhilfe Südniedersachsen e. V. (JSN), dort arbeiten Fachkräfte aus dem psychologischen und pädagogischen Bereich. Im Auftrag des Jugendamts erarbeitet das Team pro Fall eine Stellungnahme, ob die Anspruchsvoraussetzungen für Eingliederungshilfe gegeben sind und ob Schulbegleitung die geeignete Maßnahme ist. Neben der Diagnostik des psychischen Störungsbildes erfolgt eine eher sozialpädagogisch angelegte Prüfung der Teilhabegefährdung, bei der Kontextmerkmale eine zentrale Rolle spielen. Zur Klärung dieser Fragen führen die Mitarbeiter/innen Gespräche mit den Eltern und dem Kind oder Jugendlichen und hospitieren im Unterricht. Ein wichtiges Kriterium ist dabei die soziale Integration bzw. deren Gefährdung. Am Ende des Begutachtungsprozesses empfiehlt die Fachstelle dem Jugendamt eine Bewilligung oder Ablehnung des Antrages auf Schulbegleitung. Häufige Gründe, eine schulbegleitende Maßnahme nicht zu empfehlen, sind die Feststellung, dass zunächst andere Maßnahmen ergriffen werden müssten (z. B. psychotherapeutische Hilfe zur Behandlung einer Störung) oder dass die Schulen ihre eigenen Möglichkeiten noch nicht ausgeschöpft haben (z. B. die Gewährung eines Nachteilsausgleichs oder die Veranlassung einer sonderpädagogischen Überprüfung zur Beantragung sonderpädagogischer Förderung). Auch die fehlende Teilhabegefährdung aufgrund ausreichender Kompensationsmöglichkeiten einer psychischen Störung durch das Kind selbst oder sein Umfeld ist ein häufiger Grund für die Ablehnung einer Schulbegleitung. Ein letzter häufiger und besonders strittiger Grund ist die Diagnose Lernbehinderung, die weder nach dem Sozialhilferecht noch nach dem Kinder- und Jugendhilferecht die Voraussetzungen für Eingliederungshilfe erfüllt. An den genannten Gründen zeigt sich die Schwierigkeit der Abgrenzung von Leistungen, für die das Schulamt zuständig ist bzw. für die das Sozial- oder Jugendamt zuständig ist.

13 Die Fachstelle wurde eigens zu dem Zweck eingerichtet, die Anspruchsvoraussetzungen für Eingliederungshilfe nach \$35a SGB VIII für die Jugendämter in Südniedersachsen zu prüfen. 
Die letztliche Entscheidung über das Vorliegen einer seelischen Behinderung und die Bewilligung der Schulbegleitung trifft das Jugendamt in einer Helferkonferen₹: Grundlage für die Entscheidung ist die Einschätzung der Jugendamtsmitarbeiter/innen und die Stellungnahme der Fachstelle, die auch Empfehlungen zur Hilfeplanung (Umfang und Dauer, erforderlichen Qualifikation „,geeignete Person“ oder „pädagogische Fachkraft" und den Aufgaben/Zielen der Schulbegleitungsmaßnahme) enthält. Wird der Antrag bewilligt (meist für ein Schuljahr, bei Autismus oft auch gleich für zwei), ergeht ein positiver Bescheid an die Eltern, in dem die Rahmenbedingungen und Ziele der Maßnahme festgeschrieben sind. Ziele können z. B. Aufmerksamkeitssteuerung, Schutz vor Eigen- oder Fremdgefährdung oder Hilfe zur Entwicklung sozialkompetenten Verhaltens, auf jeden Fall die Verselbständigung des Schülers/der Schülerin sein. Die nächsten Schritte sind für Schulbegleitungen über die Jugendämter die gleichen wie für Schulbegleitungen über die Sozialämter: Den Eltern wird eine Auswahl an Trägern empfohlen, die Schulbegleiter/innen beschäftigen, bzw. im Fall des Jugendamts (Stadt) sucht das Amt selbst eine Person für die Schulbegleitung, die sich von einem Träger anstellen lässt.

Die Träger bieten teilweise unterschiedliche Möglichkeiten der Beschäftigung (sozialversicherungspflichtige Anstellung oder Honorartätigkeit). Freiwillige (etwa im Rahmen eines Freiwilligen Sozialen Jahres oder Bundesfreiwilligendienstes) werden von den meisten Trägern nicht als Schulbegleitung eingesetzt und das Eltern-Arbeitgeber-Modell wird in der Region Göttingen inzwischen nur selten praktiziert. Der zahlenmäBig größte Träger für Schulbegleitung ist Haus mit vielen Etagen, gefolgt von der JSN. Geringe Zahlen an Schulbegleitungen in der Region Göttingen werden außerdem durchgeführt von der Selbsthilfe Körperbehinderter, der Lebenshilfe Northeim, dem DRK Northeim und der Lebenshilfe Osterode. Alle Träger verfügen über einen Pool an Schulbegleitungen, die bereits erfolgreich ein Bewerbungsgespräch absolviert haben. Da für Schulbegleitung im Regelfall keine formale Qualifikation vorausgesetzt wird, bleibt den Trägern überlassen, wie sie die Eignung der Bewerber/innen einschätzen. Die JSN bspw. hat ein zweistufiges Bewerbungsverfahren, in dem nach einem Erstgespräch, das vor allem der Information der Bewerber/innen dient und einem zweiten Gespräch, in dem Fallbeispiele aus der Schulbegleitung durchgespielt werden, erst über die grundsätzliche Eignung und die Aufnahme in den Pool entschieden wird.

Das Jugendamt (Stadt) bzw. die Träger veranlassen ein Treffen zwischen der Person aus dem Pool, die sie im Einzelfall für geeignet halten, mit dem Kind oder Jugendlichen und seinen Eltern. Teilweise ist, bevor eine Entscheidung über die Zusammenarbeit getroffen wird, auch eine Hospitation im Unterricht üblich. Die eigentliche Einarbeitung erfolgt dann durch die Klassenleitung des Kindes oder Jugendlichen, wobei bei Jugendamtsfällen im Rahmen des Hilfeplanverfahrens auch Mitarbeiter/innen der Fachstelle oder die zuständigen Amtsmitarbeiter/innen in der ersten Zeit im Unterricht hospitieren, um ggf. Hilfestellung und Anleitung zur Ausführung der Schulbegleitung zu geben. In einem Hilfeplangespräch, an dem Jugendamtsmitarbeiter/innen, Mitarbeiter/innen der Fachstelle, die Schulbegleitung, die Eltern, die Schule und teilweise auch das Kind/die Jugendliche teilnehmen, werden zu Beginn der Maßnahme deren Ziele besprochen und festgehalten. Zur Qualitätssicherung im Verlauf der Maßnahme und zur Entscheidung über eine Verlängerung der Maßnahme finden weitere Hilfeplangespräche statt, an denen neben der Schulbegleitung selbst üblicherweise die Klassenleitung, die Eltern sowie Amtsmitarbeiter/innen und ggf. Mitarbeiter/innen der Fachstelle teilnehmen. Einige Träger bieten ihren Schulbegleiter/innen - auf freiwilliger Basis - regelmäßige Austauschmöglichkeiten (auch mit anderen Schulbegleiter/innen) sowie fachliche Schulungen an. Für alle Schulbegleiter/innen, die über das Jugendamt finanziert werden, bietet außerdem die Fachstelle für Diagnostik der JSN Fachberatung an, in denen einerseits zu Störungsbildern informiert wird und andererseits Austausch über eigene Fälle stattfinden kann.

\subsubsection{Zentrale Diskussionspunkte zu Schulbegleitung im Kontext von Inklusion}

Wenn man von den Broschüren der Träger für Schulbegleitung absieht, fällt auf, dass sich in so gut wie jeder Publikation zum Thema Schulbegleitung kritische Anmerkungen finden. Häufig wird eine Änderung bestimmter Aspekte der Maßnahme gefordert (z. B. Dworschak, 2010; Niedermayer, 2009) und eine Änderung von Schule dahingehend, dass sie Inklusion auch ohne personenbezogene Einzelfallhilfe gewährleistet (z. B. AGJ, 2014; Bloemer-Hausmanns, 2014; Böing, 2013; Knuf, 2013; Thies, 2014).

Kritik an speziellen Aspekten der Maßnahme bezieht sich häufig darauf, dass für Schulbegleitung keine formale Qualifikation vorausgesetzt ist und die pädagogische Qualität der Arbeit deshalb nicht sichergestellt ist (z. B. AGJ, 2014; Böing, 2013; Keil et al., 2010). Problematisiert werden in diesem Zusammenhang auch die geringe Entlohnung von Schulbegleiter/innen und die „prekäre Beschäftigungssituation“ (Heinrich \& 
Lübeck, 2013, S. 93). Aufgrund der Bindung des Arbeitsverhältnisses an eine Maßnahme sind die Vertragslaufzeiten in der Regel kurz und die Bezahlung bei eigener Krankheit oder Krankheit des begleiteten Kindes und in den Ferien mitunter nicht sichergestellt. Hinzu kommt, dass laufende Maßnahmen abgeändert oder sogar beendet werden können, etwa wenn das begleitete Kind umzieht oder vorübergehend in eine stationäre Einrichtung wechselt. Eine Beendigung oder Reduzierung der Maßnahme erfolgt auch dann, wenn Schulbegleitung das ihr zugrunde liegende Ziel der Eingliederung erreicht hat und das Kind sie nicht mehr oder nicht mehr im selben Maße benötigt (vgl. Henn et al., 2014). Nicht nur aus Perspektive der Schulbegleiter/innen können diese Umstände problematisch sein, sie können sich auch kontraproduktiv auf die Effektivität der Maßnahme auswirken. Schwierige Arbeitsumstände können die Fluktuation von Schulbegleiter/innen erhöhen, wodurch mitunter die Kontinuität fehlt, die für den Aufbau einer vertrauensvollen Beziehung zum begleiteten Kind nötig ist. Die Zielsetzung von Schulbegleitung, sich selbst überflüssig zu machen, erscheint paradox angesichts des Umstands, dass vielen Schulbegleiter/innen in diesem Fall der Verlust ihrer Arbeit droht.

Kritisch diskutiert wird darüber hinaus die Abgrenzung des Aufgabenbereichs, der für Schulbegleitung vorgesehen ist. Eingliederungshilfe hat soziale Teilhabe zum Ziel und nicht, wie sonderpädagogische Förderung, die Erreichung von Bildungszielen. Schulbegleiter/innen sind insofern ganz grundsätzlich nicht dafür vorgesehen, als Lernhelfer/innen zu fungieren. Während Schulbegleiter/innen keine unterrichtlichen Aufgaben wahrnehmen dürfen, die im weiteren Sinne zum Kernbereich der Schule gehören (Kißgen et al., 2013) bzw. keine Aufgaben, „die den Kernbereich der pädagogischen Arbeit der Lehrkräfte betreffen“ (NLQ, 2016), sind gleichzeitig Hilfen, die dem Kind oder der/dem Jugendlichen ermöglichen, die unterrichtlichen Lernangebote wahrzunehmen, nicht nur erlaubt, sondern vorgesehen (Greß, 2014). Es verwundert daher nicht, dass die Abgrenzung von Schulbegleiteraufgaben zu unterrichtlich-pädagogischen Tätigkeiten als unscharf und unpraktikabel kritisiert wird (z. B. Dworschak, 2012b; siehe auch Conée \& Ranscht, 2012). Dworschak (2012c) fordert deshalb die Ansiedelung der Maßnahme im Kultusbereich.

Im Zusammenhang mit der schulexternen Verortung der Maßnahme und der unklaren Rollenkonzeption von Schulbegleiter/innen steht auch Kritik an der unzureichenden Einbettung von Schulbegleiter/innen in schulische Kooperationsstrukturen (Lübeck, 2016b). Weiterreichende Forderungen beziehen sich auf die Abschaffung von Schulbegleitung als personengebundener Maßnahme (z. B. AGJ, 2014; Fischer, 2011; siehe auch Schöler, 2002). Dabei wird z. B. vorgeschlagen, Schulbegleiter/innen fest an Schulen einzustellen und je nach Bedarf zur Unterstützung einzelner Schüler/innen oder ganzer Schülergruppen einzusetzen (AGJ, 2014; Fischer, 2011; Knuf, 2013; Lindmeier \& Polleschner, 2014; Thies, 2014). Eine solche Lösung soll auch der möglichen Stigmatisierung einzelbegleiteter Kinder entgegenwirken (Böing, 2013; Köpfer, 2013a, 2013b). Lübeck (2016a) benennt als Risiken individueller Schulbegleitung die Abnahme des Verantwortungsgefühls der zuständigen Lehrkraft, die Isolation des begleiteten Kindes von der Klasse und eine Abhängigkeit des Kindes von der Schulbegleitung. Folgerichtig fragt die Autorin, inwieweit Schulbegleitung überhaupt eine zielführende Maßnahme zur Umsetzung von Inklusion ist, und verweist auf die Möglichkeit, an Regelschulen entsprechende Ressourcen wie an Förderschulen bereitzustellen - personell wie fachlich um den individuellen Bedürfnissen aller Schüler/innen gerecht zu werden (Lübeck, 2016a, 2016b).

\subsection{Forschungsstand zu Schulbegleitung}

Im Folgenden wird der aktuelle Stand der Forschung ${ }^{14}$, die sich spezifisch auf Schulbegleitung bezieht, ausführlich aufgearbeitet. Da die Anzahl verfügbarer Studien sehr überschaubar ist, bleiben viele Fragen, insbesondere solche, die über rein deskriptive Zustandsbeschreibungen hinausgehen, offen. Aus diesem Grund werden in kürzerer Form zusätzlich wichtige Befunde internationaler Forschung zu unterrichtlichen Assistenzkräften dargestellt. Dabei muss beachtet werden, dass die gesetzlichen Grundlagen und darauf aufbauend die Gestaltungsvorgaben für Schulbegleitung national verschieden sind, d. h., Schulbegleitung in der hiesigen Form existiert so in keinem anderen Land. Dennoch erstaunt, dass zwar häufig ein Forschungsdesiderat beklagt, aber eher selten auf internationale Forschung Bezug genommen wird, denn das nähere Studium internationaler Forschung offenbart erstaunlich viele Parallelen zwischen Schulbegleiter/innen und dem Assistenzpersonal, das in zahlreichen westlichen Ländern zur Unterstützung im Klassenraum einge-

14 Die letzte Aktualisierung erfolgte im März 2016. 
setzt wird. Von der Erschließung existenter Forschung über den Einsatz und die Wirkungen dieses Personals kann deshalb ein lohnenswerter Erkenntnisgewinn für die Herausforderungen im Kontext von Schulbegleitung erwartet werden.

Um beiden Aspekten - der Eigenart deutscher Schulbegleitung und der Vergleichbarkeit mit Schulassistenz in anderen Ländern - Rechnung zu tragen, ohne dabei den jeweiligen Bezugsrahmen aus den Augen zu verlieren, werden im Folgenden nationale Schulbegleitungsforschung in ausführlicher Form und zentrale internationale Forschungsergebnisse zu Assistenzpersonal separat vorgestellt.

\subsubsection{Nationale Befunde}

Zum Thema Schulbegleitung existieren bislang keine großangelegten Studien im deutschen Raum. Mittels verschiedener Stichwortrecherchen in relevanten Datenbanken (ERIC, FIS Bildung, pedocs, SOLIS, PsycINFO, Psyndex, Pubpsych), dem Studium der Literaturlisten einschlägiger Texte sowie der Publikationslisten einzelner Forscher/innen fanden sich insgesamt 12 Studien, die Schulbegleitung zum Gegenstand haben (Beck et al., 2010; Deger et al., 2015; Dworschak, 2012b, 2012c; Heinrich \& Lübeck, 2013; Henn et al., 2014; Keil et al., 2010; Kißgen et al., 2013; Lindmeier et al., 2014; Lübeck \& Heinrich, 2016; Wohlgemuth, 2009; Zauner \& Zwosta, 2014) sowie drei Sekundäranalysen, in denen Daten einer umfassenden Studie über den Förderschwerpunkt Geistige Entwicklung hinsichtlich schulbegleiteter Schüler/innen ausgewertet wurden (Dworschak, 2012a, 2014, 2015), und eine Studie, in der Schulbegleitung als Teilaspekt von Inklusion untersucht wurde (Böing, 2013). Nicht berücksichtigt sind hier Studienabschlussarbeiten, die vereinzelt in Literaturlisten erscheinen ohne im Fließtext ausgeführt zu werden. Die Ergebnisse bzw. Zwischenergebnisse bei laufenden Projekten sind überwiegend in Fachzeitschriften für Sonder- oder Heilpädagogik veröffentlicht, nur eine (kleinere) Studie wurde in einer allgemeinen bildungswissenschaftlichen Zeitschrift veröffentlicht (Heinrich \& Lübeck, 2013) und eine stammt aus dem Bereich der Kinder- und Jugendpsychiatrie (Henn et al., 2014 bzw. Fegert, Ziegenhain \& Besier, 2016). Die Studien von Böing (2013) sowie von Lübeck und Heinrich (2016) sind als Monographien bzw. Teile von Monographien erschienen und für vier Projekte (Deger et al., 2015; Dworschak, 2012c; Keil et al., 2010; Lindmeier et al., 2014) sind lediglich online Ergebnisberichte verfügbar. Die Analyse der Schulbegleiterdaten innerhalb des Projekts von Keil et al. (2010) ist in Form einer umfangreichen Diplomarbeit geleistet worden (Wohlgemuth, 2009), die nicht veröffentlicht ist, mir aber leihweise zur Verfügung gestellt werden konnte.

Alle erwähnten Studien untersuchen den Status Quo, unter ihnen finden sich weder eine Längsschnittnoch eine Interventionsstudie. In den meisten Projekten werden deskriptive Daten abgefragt, die die Maßnahme Schulbegleitung in einer bestimmten Region abbilden: Aktuelle Anzahl an Schulbegleitungen und Entwicklung der Anzahl im Zeitverlauf, Daten zu den Maßnahmen (Dauer, Umfang), Merkmale der begleiteten Schüler/innen (Schulform, Geschlecht, Alter, Behinderungsart), Merkmale von Schulbegleiter/innen (Qualifikation, Alter, Geschlecht) und Tätigkeitsfelder. In einzelnen Studien wird auch nach der Einschätzung des Erfolgs der Maßnahme gefragt. Zwei umfangreichere Studien sind von Trägern in Auftrag gegeben (Deger et al., 2015; Dworschak, 2012c) und beziehen ihre Stichprobe nur aus deren Schulbegleiter/innen. Einige andere Studien (Henn et al., 2014; Keil et al, 2010; Kißgen et al., 2013) haben Praxisanwendungen wie die Entwicklung von Qualifizierungsprogrammen für Schulbegleiter/innen zum Ziel. Besonders ambitioniert in dieser Hinsicht war das Modellprojekt QuaSI von Keil et al. (2010), das inzwischen allerdings aus Kostengründen eingestellt wurde (A. Friedemann, persönl. Mitteilung, 24.02.2016). Nur die Einzelfallstudien von Heinrich \& Lübeck (2013) bzw. Lübeck und Heinrich (2016) und die Dokumentation von Böing (2013) beziehen sich ausschließlich auf qualitative Daten, die Studien von Deger et al. (2015), Lindmeier et al. (2014) sowie von Zauner und Zwosta (2014) auf quantitative und qualitative Daten. Die meisten Studien sind als Fragebogenstudien angelegt, die sich an Schulbegleiter/innen und/oder Vertreter/innen der Schule oder der Träger wenden. In zwei Fällen wurden dabei auch die Eltern und Klassenleitungen und in einem davon auch die begleiteten Schüler/innen selbst einbezogen (Wohlgemuth, 2009; Zauner \& Zwosta, 2014). Die qualitativen Studien(anteile) von Deger et al. (2015), Lindmeier et al. (2014) und Böing (2013) dokumentieren Diskussionen verschiedener Beteiligtengruppen (Schüler/innen, Eltern, Schulbegleiter/innen, Schulvertreter/innen, Schulamtsvertreter/innen). Regionale Schwerpunkte in der Schulbegleitungsforschung liegen in Bayern (Beck et al., 2010; Dworschak, 2012a, 2012b, 2012c, 2014, 2015) und Baden-Württemberg (Deger et al., 2015; Henn et al., 2014; Zauner \& Zwosta, 2014). Regionalbezogene Daten werden darüber hinaus für Nordrhein-Westfalen (Kißgen et al., 2013), die Region Hannover (Lindmeier et al., 2014) und Thüringen (Wohlgemuth, 2009) berichtet. 
In diesem Kapitel werden die verfügbaren Ergebnisse der bisherigen Studien zu Schulbegleitung systematisch anhand folgender Unterpunkte zusammengefasst:

- Schulformen und Schülermerkmale

- Dauer, Umfang und Träger der Maßnahme

- Merkmale und Qualifikation von Schulbegleiter/innen

- Tätigkeitsfelder von Schulbegleiter/innen

- Kooperation und Evaluation

Um eine Vorstellung von der relativen Bedeutung der Zahlen zu erhalten, die in den empirischen Arbeiten berichtet werden, werden an dieser Stelle zunächst übergeordnete Zahlen zu Schülerzahlen, Schulformen und Eingliederungshilfe vorgestellt, bevor die Darstellung des Forschungsstandes anhand der aufgeführten Punkte erfolgt. Der Vergleichbarkeit halber werden schulbezogene Zahlen, soweit möglich, einheitlich für das Schuljahr 2013/14 berichtet. Für die Folgejahre sind noch nicht für alle relevanten Bereiche Zahlen veröffentlicht. Die Gesamtzahl von Schüler/innen an allgemeinbildenden Schulen beträgt im Schuljahr 2013/14 etwa 8.4 Millionen. Davon besuchen etwa 32\% die Grundschule und 28\% ein Gymnasium. An Realschulen lernen 12\%, an Hauptschulen 7\% und an Gesamtschulen oder anderen Schulen mit mehreren Bildungsgängen 14\% der Schüler/innen. Förderschulen werden von 4\% der Schüler/innen besucht. Die übrigen Schüler/innen verteilen sich auf freie Schulkindergärten, freie Schulen und Abendschulen (StB, 2016).

Insgesamt erhielten im Schuljahr 2013/14 rund 500500 Schüler/innen sonderpädagogische Unterstützung, was einer Förderquote (dem Anteil dieser Gruppe an der gesamten Schülerschaft) von bundesweit 6.8\% entspricht (Klemm, 2015; in Niedersachen: 5.3\%). Zwei Drittel aller Schüler/innen mit sonderpädagogischem Förderbedarf sind männlich (Autorengruppe Bildungsberichterstattung, 2014). Der häufigste Förderschwerpunkt ist Lernen (38.8\%), gefolgt von Geistige Entwicklung (16\%) und Emotionale und soziale Entwicklung (15.2\%, Klemm, 2015). Insgesamt werden 4.7\% der gesamten Schülerschaft an Förderschulen unterrichtet (Exklusionsquote, Niedersachsen: 4.1\%) und 2.1\% als Schüler/innen mit sonderpädagogischem Förderbedarf an Allgemeinen Schulen (Inklusionsquote; Niedersachen: 1.2\%). Die Exklusionsquote ist in den letzten Jahren nahezu konstant geblieben, obwohl sich die Inklusionsquote erhöht hat, was sich mit dem parallelen Anstieg der Förderquote erklären lässt (Klemm, 2015; Malecki, 2013). Von allen Schüler/innen mit sonderpädagogischer Förderung werden 31.4\% inklusiv beschult (Klemm, 2015). Aus dieser Gruppe besuchen 44.3\% die Grundschule, 28\% eine Integrierte Gesamtschule oder andere Schularten mit mehreren Bildungsgängen und 11.3\% eine Hauptschule (KMK, 2016). Der häufigste Förderschwerpunkt dieser Schüler/innen ist Lernen (43.6\%), gefolgt von Emotionale und soziale Entwicklung (24.8\%) und Sprache (13.4\%). Ein besonders hoher Inklusionsanteil findet sich unter den Schüler/innen mit dem Förderschwerpunkt Emotionale und soziale Entwicklung (53\%, ebd.).

Genaue Angaben zu Schüler/innen mit Behinderung sind nicht leicht zu finden. Laut Deutschem Gesundheitsbericht (RKI, 2015) liegt bei $2 \%$ aller Kinder und Jugendlichen unter 18 Jahren eine amtlich anerkannte Behinderung vor, der überwiegende Teil ist schwerbehindert. Ihr Anteil liegt also in jedem Fall deutlich unter dem der Schüler/innen mit Förderbedarf. Zur absoluten Häufigkeit von Schulbegleitung existieren keine offiziellen Zahlen. Der Sozialstatistik lässt sich aber entnehmen, dass im Jahr 2013 insgesamt 46053 Kinder und Jugendliche schulbezogene Eingliederungshilfen ${ }^{15}$ erhielten (StB, 2015a, 2015b). Darunter fallen neben Schulbegleitung auch z. B. der Besuch Heilpädagogischer Tagesstätten (Greß, 2014), es kann aber davon ausgegangen werden, dass Schulbegleitung den Großteil davon ausmacht (Autorengruppe Bildungsberichterstattung, 2014). Gegenüber dem Jahr 2010 hat sich die Zahl der schulbezogenen Eingliederungshilfen damit nahezu verdoppelt, damals betrug sie 26212 (StB, 2012, 2013). Das Verhältnis von Schüler/innen, die Eingliederungshilfe erhalten, zu Schüler/innen mit sonderpädagogischem Förderbedarf liegt damit bei 1:11. Legt man die Gesamtzahl von Schüler/innen an allgemeinbildenden Schulen zugrunde, kommt auf 182 Schüler/innen ein/e Schüler/in mit Eingliederungshilfe.

15 Bei der Eingliederungshilfe nach SGB VIII habe ich zur Berechnung nur die „Hilfen zur angemessenen Beschulung außerhalb von Einrichtungen“ berücksichtigt, da ,innerhalb von Einrichtungen“ bedeutet, dass der/die Schüler/in in einer Einrichtung lebt, was es wahrscheinlich macht, dass die Eingliederungshilfe der Deckung der Einrichtungskosten dient und nicht dem Einsatz einer Schulbegleitung. 
Schulformen und Schülermerkmale. Dem Mangel an offiziell verfügbaren statistischen Daten zu Schulbegleitung wird in regionalbezogenen Studien ebenfalls meist damit begegnet, eigens recherchierte bzw. von den Ämtern erfragte Zahlen abzubilden. So wurden in der Region Hannover im Schuljahr 2013/14 insgesamt 624 Schüler/innen begleitet, was einem Anteil von 10\% aller Schüler/innen mit sonderpädagogischen Förderbedarf entspricht (Lindmeier et al., 2014). Mehrere Autor/innen berichten den Anstieg von Schulbegleitungen sowohl an Förder- als auch in Allgemeinen Schulen (Beck et al., 2010; Dworschak, 2012b, 2012c; Kißgen et al., 2013; Lindmeier et al., 2014). Kißgen et al. (2013) verzeichnen einen Anstieg um das 30 fache in nordrhein-westfälischen Förderschulen zwischen den Schuljahren 2000/01 und 2010/11. Zum Verhältnis von Schulbegleitung an Allgemeinen und an Förderschulen werden unterschiedliche Zahlen berichtet. In Anbetracht der Schülerklientel wäre es plausibel anzunehmen, dass Schulbegleitung häufig an Förderschulen geleistet wird, allerdings ist die Notwendigkeit der Maßnahme aufgrund anderer Rahmenbedingungen dort grundsätzlich weniger gegeben als an Allgemeinen Schulen (vgl. Dworschak, 2015). Wohlgemuth (2009) berichtet für Thüringen von einer überwiegenden Inanspruchnahme von Schulbegleitungen an Grundschulen (45\%) und Förderschulen (knapp 40\%), in der aktuellen Studie von Deger et al. (2015) zu Schulbegleitung in der Region Hannover sind Grundschulen mit 70\% noch stärker vertreten und Förderschulen mit 16\% in erheblich geringerem Maße. Womöglich hat die steigende Inklusionsquote zu einem höheren Anteil von Schulbegleitungen an Allgemeinen Schulen geführt. Zumindest liefert die Studie von Dworschak (2015) den Hinweis, dass Schüler/innen mit sonderpädagogischem Förderbedarf an Allgemeinen Schulen weitaus häufiger eine Schulbegleitung erhalten als an Förderschulen; in der (bayrischen) Stichprobe der Studie werden Schüler/innen bestimmter Förderschwerpunkte an Allgemeinen Schulen zu 95\% schulbegleitet und an Förderschulen nur zu 15\%.

Die Förderschwerpunkte der schulbegleiteten Schüler/innen wurden nur in einer Studie (Wohlgemuth, 2009) erhoben, am häufigsten wurde der Schwerpunkt Emotionale und soziale Entwicklung genannt, gefolgt von Motorische Entwicklung, Sprache, Geistige Entwicklung und Lernen, wobei viele Kinder und Jugendliche mehr als einem Förderschwerpunkt zugeordnet wurden. Die Angaben dazu, ob die Maßnahme im Rahmen der Jugendhilfe oder der Sozialhilfe bewilligt wurde, lassen darauf schließen, dass den Anträgen seelische Behinderungen und körperliche oder geistige Behinderungen aktuell etwa zu gleichen Anteilen zugrunde liegen (Deger et al., 2015; Lindmeier et al., 2014). Wohlgemuth (2009) fand demgegenüber einen höheren Anteil von Bewilligungen durch das Sozialamt. Generell wird Schulbegleitung an Allgemeinen Schulen häufiger als Jugendhilfe und an Förderschulen häufiger als Sozialhilfe - und hierbei häufiger aufgrund körperlicher als aufgrund geistiger oder Sinnesbehinderung - geleistet (Deger et al., 2015; Henn et al., 2014; Lindmeier et al., 2014). Unter den Diagnosen seelischer Behinderung finden sich gehäuft Autismus, ADHS und Störungen des Sozialverhaltens (Henn et al., 2014; Zauner \& Zwosta, 2014; siehe auch Wohlgemuth, 2009). Schulbegleitungen an Allgemeinen Schulen und an Förderschulen scheinen sich auch hinsichtlich weiterer Merkmale zu unterscheiden: Für bayrische Schüler/innen mit dem Förderschwerpunkt Geistige Entwicklung fand Dworschak (2012a), dass diejenigen unter ihnen, die Schulbegleitung an Förderschulen erhielten, schwerere Behinderungsgrade aufwiesen als diejenigen unter ihnen, die Schulbegleitung an einer Allgemeinen Schule erhielten (siehe auch Dworschak, 2014). Als Beantragungsgrund für Schulbegleitung an Allgemeinen Schulen stehen die Aspekte Lernen und Integration in die Allgemeine Schule im Vordergrund, für Förderschulen wird am häufigsten Alltagsbewältigung und Verbalten genannt (Dworschak, 2012a, 2014). Pflege wird als Beantragungsgrund in beiden Fällen nur von etwa $10 \%$ der Befragten angegeben (Dworschak, 2012a). Diese Zahlen zeigen, dass Schulbegleitung nicht nur in der Konsequenz, sondern auch in der Vorstellung der Beteiligten offensichtlich häufig als Inklusionshilfe dient. Kritisch zu bewerten ist dabei die Tatsache, dass nicht nur der tatsächliche Unterstützungsbedarf die Wahrscheinlichkeit erhöht, dass ein/e Schüler/in Schulbegleitung erhält (Dworschak, 2014), sondern aufgrund der Beantragung durch die Erziehungsberechtigten auch familiäre Bedingungen Einfluss haben können (siehe hierzu Dworschak, 2015).

Überproportional häufig erhalten männliche Schüler Schulbegleitung (Deger et al., 2015; Dworschak, 2014; Wohlgemuth, 2009), besonders im Rahmen der Jugendhilfe (Deger et al., 2015). Das Durchschnittsalter aller aktuell schulbegleiteten Schüler/innen wurde in zwei Studien erhoben und betrug etwa 10 bzw. 11 Jahre (Henn et al., 2014; Wohlgemuth, 2009). Im Schnitt sind die Schüler/innen, die im Rahmen von Jugendhilfe Schulbegleitung erhalten, zum Zeitpunkt der Antragstellung älter als diejenigen, die Schulbegleitung im Rahmen von Sozialhilfe erhalten (Deger et al., 2015). 
Dauer, Umfang und Träger der Maßnahme. Zwar finden sich in mehreren Studien Angaben zur Maßnahmendauer, meist bleibt aber unklar, ob der aktuelle Bewilligungszeitraum gemeint ist oder die Gesamtdauer der Maßnahme über verschiedene Bewilligungszeiträume. Deger et al. (2015) geben für die Region Hannover an, dass der Bewilligungszeitraum meist ein Jahr beträgt, im Rahmen der Jugendhilfe manchmal auch weniger. Für Anträge sowohl im Jugend-als auch im Sozialhilfebereich berichten die Autor/innen eine Bewilligungsquote von über $80 \%$, wobei von der Beantragung bis zur Entscheidung mehrere Wochen oder sogar Monate vergehen (ebd.). Von vergleichbaren Zeiträumen berichtet auch Wohlgemuth (2009), die darüber hinaus feststellt, dass für die meisten der begleiteten Schüler/innen (80\% bzw. $75 \%$ ) ein Folgeantrag und eine Weiterbegleitung durch die gleiche Person geplant sind. Der Stundenumfang, in dem Schulbegleitung bewilligt wird, scheint generell stark zu variieren. Für verschiedene Stichproben wurde festgestellt, dass mehrheitlich Schulbegleitung für die gesamte Unterrichtszeit geleistet wird, für manche Schüler/innen aber auch nur für wenige Stunden (Dworschak, 2012b; Lindmeier et al., 2014; Wohlgemuth, 2009). Angaben zur ausführenden Einrichtung der Maßnahme finden sich bei Wohlgemuth (2009) und Deger et al. (2015), demnach werden überwiegend freie Träger beauftragt, die die Schulbegleiter/innen sozialversicherungspflichtig oder auf Honorarbasis beschäftigen. Das Eltern-Arbeitgeber-Modell scheint generell selten vorzukommen (Deger et al., 2015; Dworschak 2012c; Henn et al., 2014; Wohlgemuth, 2009), noch seltener die Krankenkasse als Finanzier (Henn et al., 2014). Auch die Begleitung von mehr als einem Kind oder Jugendlichen durch dieselbe Person scheint ein seltener Sonderfall zu sein (Lindmeier et al., 2014; Wohlgemuth, 2009; Zauner \& Zwosta, 2014).

Merkmale und Qualifikation von Schulbegleiter/innen. Personenbezogene Daten, die zu Schulbegleiter/innen selbst erhoben wurden, beziehen sich vor allem auf ihren Qualifikationshintergrund. Dem vorangestellt seien die wenigen anderen verfügbaren Angaben: In der Stichprobe von Schulbegleiter/innen an Allgemeinen Schulen von Henn et al. (2014) betrug der Frauenanteil 86\% und das Durchschnittsalter lag bei 41 Jahren bei einer Spanne von 17 bis 69 Jahren. Wohlgemuth (2009) findet ähnliche Ergebnisse für Schulbegleiter/innen in Allgemeinen und in Förderschulen. Eine relativ gleichmäßige Streuung über alle Altersklassen ab 20 Jahren berichten Zauner und Zwosta (2014). Zur Tätigkeitsdauer als Schulbegleitung geben in den Stichproben von Dworschak (2012b, 2012c) je über 60\% der befragten Personen drei Jahre oder kürzer an. Insgesamt arbeiten 32\% bzw. 42\% noch nicht länger als ein Jahr als Schulbegleitung (ebd.).

Zur Qualifizierung von Schulbegleiter/innen wurde in den meisten Studien nach Abschluss einer Berufsausbildung oder eines Studiums im pädagogischen Bereich (z. B. Erzieher/innen, Sozialpädagogik, Heilpädagogik), seltener auch im pflegerischen Bereich (z. B. Heilerziehungspflege), gefragt. Nimmt man beide Bereiche zusammen, ergibt sich in den meisten Stichproben eine Fachkraftquote um die 50\% (Beck et al., 2010; Dworschak 2012b; Henn et al., 2014; Wohlgemuth, 2009). Wohlgemuth (2009) findet, dass Schulbegleiter/innen an Sekundarschulen tendenziell über höhere Schulabschlüsse verfügen als an Grund- und Förderschulen. Für die Region Hannover ergab eine Anfrage bei den Ämtern, dass in der Jugendhilfe nur pädagogisch qualifiziertes Personal eingesetzt werde, in der Sozialhilfe dagegen auch ungelernte Kräfte (Lindmeier et al., 2014). Ähnliche Angaben, nämlich dass z. B. junge Erwachsene im Freiwilligen Sozialen Jahr nur in der Sozialhilfe eingesetzt werden, finden sich auch an anderer Stelle (Deger et al., 2015); insgesamt scheint die Personengruppe der Freiwilligen aber keinen großen Anteil der Schulbegleiter/innen zu stellen (siehe auch Wohlgemuth, 2009). In Relation zu anderen Studienergebnissen, auch innerhalb des gleichen Bundeslandes (siehe Beck et al., 2010; Henn et al., 2014), fallen die Fachkraftquote bzw. die Quote qualifizierter Hilfskräfte in den Stichproben von Zwosta und Zauner (2014) und Dworschak (2012c) mit 80\% bzw. 86\% ungewöhnlich hoch aus. Beide Stichproben sind trägerbezogen, was mindestens in einem der beiden Fälle der wahrscheinliche Grund für diese hohe Zahl ist, da dieser Träger nur einschlägig qualifizierte Bewerber/innen einsetzt (Zwosta \& Zauner, 2014).

Die Mehrheit der befragten Schulbegleiter/innen fühlt sich generell ausreichend qualifiziert für ihre Tätigkeit (Dworschak 2012b; Wohlgemuth, 2009). Die Angaben zu Weiterbildungswünschen deuten darauf hin, dass sich die Befragten vor allem im Bereich didaktischer Aufgaben und bei der gezielten Kompetenzförderung des begleiteten Kindes oder Jugendlichen mehr Wissen wünschen, während dies für die Unterstützung bei lebenspraktischen Anforderungen weniger für nötig erachtet wird (Dworschak, 2012b). 
Tätigkeitsfelder von Schulbegleiter/innen. Aus der Abfrage von Tätigkeitsfeldern von Schulbegleitungen ergibt sich in allen Studien ein breites Spektrum an Aufgaben. Wie es ein Teilnehmer bei einer Gruppendiskussion über Schulbegleitung ausdrückte, übernehmen Schulbegleiter/innen „im Prinzip alles vom Fahren des Rollstuhls über Wickeln über Füttern über Material erstellen, also alles, was letztendlich, gehändelt " werden muss" (Lindmeier et al., 2014, S. 25). Zur Ergebnisdarstellung nehmen die Autor/innen unterschiedliche Gliederungen der Tätigkeitsfelder vor, z. B. die Einteilung in „Unterstützung bei lebenspraktischen Anforderungen“, „Unterstützung bei Lernvorhaben in der Gruppe“, „Einzelförderung nach fachlicher Anleitung“ und „Sonstige Aufgaben“ (Dworschak, 2012c; Lindmeier et al., 2014). In der Region Hannover wird in den Leistungsvereinbarungen der Ämter zwischen „direkten Leistungen“, d. h. Aufgaben, die im direkten Kontakt mit dem Kind oder Jugendlichen erbracht werden, und „mittelbaren Leistungen“, wozu Vor- und Nachbereitung, Besprechungen und Fortbildungen gehören, unterschieden (Lindmeier et al., 2014). In Anlehnung an diese Strukturierung nehme ich zur systematischen Darstellung der Aufgaben von Schulbegleiter/innen eine Einteilung vor in Unterstützung bei lebenspraktischen Anforderungen, Unterstützung bei der Emotions- und Verbaltensregulation, didaktische Unterstützung und mittelbare Aufgaben, wobei ich unter mittelbare Aufgaben alle Tätigkeiten fasse, die Koordination, Dokumentation und Qualifizierung betreffen. Unterstützung bei lebenspraktischen Anforderungen bezieht sich auf pflegerische Aspekte oder Unterstützung in der Orientierung, didaktische Unterstützung bezieht sich auf Hilfe bei der Lernstoffvermittlung. An den Stichprobenbefragungen von Schulbegleiter/innen lässt sich nicht nur ein breites Aufgabenspektrum ablesen, sondern zumindest auch Tendenzen, welche Tätigkeitsbereiche häufig oder weniger häufig vorkommen. Angaben über die Häufigkeit bestimmter Tätigkeiten bzw. den Anteil der Schulbegleiter/innen, zu deren Aufgabengebiet sie gehören, liegen für folgende Stichproben vor: Beck et al. (2010) sowie Dworschak (2012b, 2012c) befragten Schulbegleiter/innen, die überwiegend für Schüler/innen mit dem Förderschwerpunkt Geistige Entwicklung tätig waren, Henn et al. (2014) befragten Schulbegleiter/innen an Allgemeinen Schulen und Wohlgemuth (2009) befragte sowohl Schulbegleiter/innen an Allgemeinen als auch an Förderschulen. Zudem finden sich zusammenfassende Tätigkeitsbeschreibungen bei Lindmeier et al. (2014) und Böing (2013) sowie eine Einzelfallanalyse (Heinrich \& Lübeck, 2013).

Vom Großteil der Schulbegleiter/innen für Schüler/innen mit dem Förderschwerpunkt Geistige Entwicklung werden vielfältige Aufgaben zur Unterstützung in lebenspraktischen Anforderungen übernommen (z. B. Hilfe bei der Fortbewegung, Hilfe beim An- und Ausziehen, Bereitlegung der Schulsachen), wobei es scheint, dass genuin pflegerische Aufgaben (Hilfe bei Waschen, Toilettengang, Essen) eher an Förder- als an Allgemeinen Schulen geleistet werden (Dworschak, 2012b, 2012c). Bei dem überwiegenden Teil von Schulbegleiter/innen an Allgemeinen Schulen scheint die Unterstützung bei lebenspraktischen Anforderungen nur eine untergeordnete Rolle zu spielen (Henn et al., 2014). Der Bereich Unterstützung bei der Emotions- und Verhaltensregulation ist dagegen in allen Stichproben stark ausgeprägt. Etwa $80 \%$ der jeweils befragten Schulbegleiter/innen leistet „Emotionale Unterstützung“ und „Unterstützung von Sozialkontakten“ (Dworschak, 2012b, 2012c; siehe auch Lindmeier et al., 2014) bzw. Unterstützung bei der „Verhaltenskontrolle“ (Wohlgemuth, 2009) bzw. unterstützt das Kind oder den Jugendlichen bei Konfliktklärungen, bei der Reduzierung des Stressniveaus oder der Selbstwertsteigerung (Henn et al., 2014). Auch die didaktische Unterstützung nimmt in allen Stichproben breiten Raum ein. Die große Mehrheit der Schulbegleiter/innen für Schüler/innen mit Förderschwerpunkt Geistige Entwicklung unterstützt (Gruppen-)Lernvorhaben in verschiedenen Fächern und ein knappes Drittel (an Förderschulen) bzw. knapp die Hälfte (an Allgemeinen Schulen) von ihnen bereitet Unterrichtsmaterialien vor (Dworschak, 2012b, 2012c). Unterrichtsplanung bzw. die Erstellung von Förderplänen gehört je nach Stichprobe bei 7\% bis 16\% der befragten Schulbegleiter/innen zu ihren Aufgaben (Beck et al., 2010; Dworschak, 2012b, 2012c). Rund 86\% der Schulbegleiter/innen in Thüringen geben an, Aufgabenstellungen anzupassen und Arbeitsmaterial zu erstellen oder zu verändern (Wohlgemuth, 2009) und über 80\% der Schulbegleiter/innen an Allgemeinen Schulen in Baden-Württemberg berichten, dass sie manchmal oder häufig Unterstützung bei Gruppenarbeiten, beim Rechnen oder Schreiben/Lesen leisten; außerdem passen gut 60\% Unterrichtsmaterial an und über die Hälfte unterstützen die Lehrkraft beim Unterrichten (Henn et al., 2014). Die Diskussionsanalyse von Lindmeier et al. (2014) offenbart, dass Schulbegleiter/innen diese Tätigkeiten häufig ohne ausreichende vorherige Anleitung ausführen. Dass didaktische Unterstützung ein zentrales Aufgabenfeld von Schulbegleitungen ist, zeigen auch die Ergebnisse der Diskussionsdokumentation von Böing (2013).

Ebenso wie die Unterstützung bei Emotions- und Verhaltensregulation und die didaktische Unterstützung fallen bei der Mehrheit der Schulbegleiter/innen in allen Stichproben auch mittelbare Aufgaben an. In der von Lindmeier et al. (2014) ausgewerteten Gruppendiskussion wird dazu kritisch angemerkt, dass diese 
Aufgabenanteile nicht immer vergütet werden. Die große Mehrheit bespricht sich mit den Eltern (Dworschak, 2012b, 2012c; Henn et al., 2014; Wohlgemuth, 2009), knapp zwei Drittel sogar häufig (Henn et al., 2014), und fast alle besprechen sich mit den Lehrkräften (Henn et al., 2014; Dworschak 2012b, 2012c). Darüber hinaus geben in der Stichprobe von Henn et al. (2014) 80\% der Schulbegleiter/innen an, manchmal oder häufig als Vermittler/innen zwischen Schule, Schüler/innen und Eltern zu fungieren. Die Teilnahme an Förderplangesprächen fällt bei etwa der Hälfte bzw. über Dreiviertel der Schulbegleiter/innen in ihr Aufgabengebiet (Dworschak, 2012b, 2012c; Wohlgemuth, 2009), die Teilnahme an Hilfeplangesprächen bei 85\% (Henn et al., 2014). Die Anteile an Schulbegleiter/innen, die an allgemeinen, nicht direkt auf den oder die begleitete/n Schüler/in bezogenen Besprechungen wie Elternabenden, Klassen- oder Lehrerkonferenzen teilnehmen, variiert zwischen rund 20\% (Dworschak 2012b; Wohlgemuth, 2009) und 48\% (Henn et al., 2014). Die Dokumentation der Maßnahme in Form von Berichten oder Protokollen übernehmen etwa ein Drittel (Dworschak 2012b, 2012c) bzw. etwa zwei Drittel der Schulbegleiter/innen (Henn et al., 2014; Wohlgemuth, 2009) und ein Drittel bis die Hälfte von ihnen bildet sich - vor allem über Angebote des Trägers fort (Dworschak, 2012b, 2012c; Wohlgemuth, 2009). In zwei Stichproben fallen bei 7\% bzw. 22\% der Schulbegleiter/innen auch fachfremde Aufgaben wie Putzen bzw. Aufräumen des Klassenzimmers an (Dworschak 2012b, 2012c).

Zuletzt sei noch auf die qualitative Fallrekonstruktion von Heinrich und Lübeck (2013) hingewiesen. Die von den Autor/innen interviewte Schulbegleiterin gibt an, einen großen Teil ihrer Zeit im Unterricht mit Häkeln zu verbringen. Diese Nebenbeschäftigung wird als Reaktion auf die Widersprüchlichkeit der Situation verstanden. Die Schulbegleiterin soll für den begleiteten Schüler ansprechbar zu sein, ihn aber nicht einnehmen, und soll gleichzeitig nicht für andere Schüler/innen tätig werden: „,Die sollten wissen, erstens ich bin für Marvin zweiten- also zuständig zweitens, ich konn auch zu den anderen Kindern kommen aber ich bin auch selbst beschäftigt. Das heißt, ich kann nicht immer gerufen werden“" (S. 100). Auf diese Weise fungiert das Häkeln „letztlich als effektives Moment der Normalisierung“ (S. 105) einer Situation, in der ihr keine einfach zu lösende Rolle zugeschrieben ist, nämlich „den Unterricht nicht zu stören, zugleich aber präsent zu sein“ (S. 106).

Kooperation und Evaluation. In keiner quantitativen Studie ist Kooperation ein Schwerpunkt, in einigen werden aber allgemeine Fragen zur Zufriedenheit mit der Zusammenarbeit sowie zur Bewertung der Maßnahme gestellt (Dworschak, 2012b, 2012c; Wohlgemuth, 2009; Zauner \& Zwosta, 2014). Eine Studie (Zauner \& Zwosta, 2014) widmet sich speziell und umfassend dem Aspekt der Bewertung der Maßnahme und wird gesondert vorgestellt, bevor abschließend kurz auf die Ergebnisse der Gruppendiskussionen zu Schulbegleitung von Böing (2013), Deger et al. (2015) und Lindmeier et al. (2014) sowie auf die Fallrekonstruktion von Lübeck \& Heinrich (2016) eingegangen wird.

Zur Bewertung des Verhältnisses zwischen Klassenleitung und Schulbegleitung finden sich Angaben bei Wohlgemuth (2009) und bei Zauner und Zwosta (2014). In der Studie von Wohlgemuth (2009) bewerten die Klassenleitungen die Zusammenarbeit mit den Schulbegleiter/innen insgesamt sehr positiv; die überwiegende Mehrheit gibt kindbezogene Informationen an die Schulbegleitung weiter. Auch die Zufriedenheitswerte der Schulbegleiter/innen bezüglich der Unterstützung durch die Lehrkräfte und die Schulleitung fallen insgesamt hoch aus. Von den befragten Schulbegleiter/innen gab jeweils der Großteil an, die Möglichkeit eines Gesprächs mit Lehrkräften (94\%), der Schulleitung (83\%) und sonderpädagogischen Fachkräften $(78 \%)$ zu haben. Außerdem erhalten knapp 70\% regelmäßige Rückmeldungen über ihre Arbeit, Supervision jedoch nur 15\%. Zauner \& Zwosta (2014) baten Eltern, Klassenleitungen und Schulbegleiter/innen um eine Bewertung der Zusammenarbeit, die insgesamt ebenfalls positiv ausfiel. Dabei äußerten sich anteilig mehr Eltern (je 100\%) als Schulbegleiter/innen (je etwa 80\%) positiv zur beidseitigen Zusammenarbeit und zum Informationsaustausch. Ebenso zeigen sich die Klassenleitungen (mit 98\%) zufriedener als die Schulbegleiter/innen (mit 87\%) mit der beidseitigen Zusammenarbeit. Dagegen empfanden 8\% der Klassenleitungen die Anwesenheit der Schulbegleiter/innen im Unterricht als störend.

Allgemeine Einschätzungen zur Bewertung der Maßnahme wurden von Wohlgemuth (2009) und Dworschak (2012b, 2012c) erfragt. Die Studie von Dworschak (2012b) zu Schulbegleitung für inklusiv beschulte Schüler/innen mit dem Förderschwerpunkt Geistige Entwicklung ergibt hohe Zufriedenheitswerte mit der Maßnahme bei fast allen Schulleitungen und Schulbegleiter/innen (je über 90\%) gegenüber einem etwas geringeren Anteil bei den Klassenleitungen und den Lehrkräften des Mobilen Sonderpädagogischen Dienstes, von denen etwa 18\% nicht zufrieden mit der Maßnahme sind. Auch bezogen auf die Integration 
des Kindes oder Jugendlichen als Effekt der Schulbegleitung zeigen sich die Klassenleitungen, die in 18\% der Fälle keinen Effekt und in 4\% der Fälle sogar einen negativen Effekt sehen, kritischer als die Schulbegleitungen. Die insgesamt hohen Zufriedenheitswerte von Schulbegleiter/innen mit der Maßnahme und dem Erfolg der Maßnahme findet Dworschak (2012c) auch in einer anderen Stichprobe. Wohlgemuth (2009) berichtet hohe Zufriedenheitswerte der Schulbegleiter/innen hinsichtlich der Inhalte ihrer Arbeit (bei vergleichsweise geringerer Zufriedenheit mit Rahmenbedingungen der Tätigkeit wie der Sicherheit des Arbeitsplatzes, den beruflichen Perspektiven und dem Gehalt). Die Klassenleitungen beurteilen die Arbeit der Schulbegleiter/innen insgesamt positiv. Bezüglich der sozialen Integration und tendenziell auch bezüglich der Schulleistungen des begleiteten Kindes oder Jugendlichen sehen sowohl die meisten Schulbegleiter/innen als auch die meisten Lehrkräfte Verbesserungen. Weiterhin findet Wohlgemuth (2009), dass die große Mehrheit der Schulbegleiter/innen (rund 80\%) den gesamten Schultag in direkter Nähe zum Kind verbringt. Die meisten der Befragten fühlen sich zwar bezüglich möglicher negativer Effekte von Schulbegleitung - wie der Beeinträchtigung von Interaktionen mit Lehrkräften und Peers und unnötiger Abhängigkeit - nicht besorgt, die Möglichkeit einer stigmatisieren Wirkung schließen viele aber nicht aus. Als Indikator für eine erfolgreiche Integration durch Schulbegleitung wurde von Dworschak (2012b) erfragt, wieviel Prozent der begleiteten Unterrichtszeit der Schüler oder die Schülerin in die Klassengemeinschaft integriert ist. Durchschnittlich liegt der Wert bei knapp 80\%, allerdings sind etwa 15\% der Schüler/innen während der begleiteten Unterrichtszeit nur die Hälfte der Zeit in die Klassengemeinschaft integriert.

Nur eine Studie widmet sich umfassend der Bewertung und der Wirkung von Schulbegleitung (Zauner \& Zwosta, 2014). Im Querschnitt wurden multiperspektivisch Eltern, Schulbegleiter/innen, Klassenleitungen sowie die begleiteten Schüler/innen selbst nach ihren Einschätzungen gefragt. Insgesamt fallen die Einschätzungen sehr positiv aus, zu bedenken ist dabei aber die vergleichsweise hohe Fachkraftquote der Stichprobe, die die Generalisierbarkeit der Ergebnisse möglicherweise einschränkt. An dieser Stelle werden deshalb vorrangig die Befunde berichtet, von denen ein Erkenntnisgewinn hinsichtlich genereller Tendenzen in der Bewertung der Maßnahme oder hinsichtlich der Kooperationsthematik erwartet werden kann. Die Zufriedenheitsrate hinsichtlich der Betreuung durch den Träger ist unter den Schulbegleiter/innen hoch (über 90\%), für nützlich werden die vom Träger angebotenen Einzelgespräche, Teamsitzungen und Schulungen aber nur von 50 bis 60\% der Befragten gehalten. Bei der Bewertung der Maßnahme als sinnvoll bzw. hilfreich äußern sich jeweils um die 90\% der befragten Schulbegleiter/innen, Lehrkräfte, Eltern und Schüler/innen zustimmend, wobei der Anteil an Zustimmung bei den Eltern am höchsten ist (97\%) und bei den Schüler/innen am niedrigsten (89\%). Die Bewertung von Grundschüler/innen fällt positiver aus als die von Sekundarschüler/innen, von denen fast die Hälfte angibt, dass ihnen die Schulbegleitung zumindest gelegentlich unangenehm war. Sowohl Schulbegleiter/innen als auch Schüler/innen geben überwiegend an, sich gegenseitig zu mögen, im Vergleich empfinden Schulbegleiter/innen ihren Schüler/innen gegenüber aber mehr Sympathie als umgekehrt. Nach Einschätzung fast aller Eltern (95\%) konnte zwischen Schulbegleiter/in und Schüler/in ein (weitgehend) vertrauensvolles Verhältnis aufgebaut werden. Die auf diese Weise erfassten Beziehungsvariablen Vertrauen und Sympathie korrelierten signifikant positiv mit der Schulbegleiterbewertung der Maßnahme als sinnvoll. Die Mehrheit der Lehrkräfte sieht keinerlei stigmatisierende Effekte von Schulbegleitung (62\%) und keinerlei Störung anderer Schüler/innen durch die Anwesenheit der Schulbegleiter/innen in der Klasse (72\%). Damit sieht allerdings immer noch ein nicht unwesentlicher Anteil zumindest eingeschränkte stigmatisierende Effekte bzw. Störungen.

Sowohl Schulbegleiter/innen, Lehrkräfte als auch Eltern geben zu hohen Anteilen (je über 80\%) an, dass sich die Schülerin oder der Schüler durch die Schulbegleitung gut entwickelt habe, dagegen sehen nur 67\% der Schüler/innen bei sich selbst eine Veränderung. Bei der Bewertung von einzelnen Effekten von Schulbegleitung liegen die Zustimmungsquoten jeweils niedriger. Erfragt wurden, ob das Kind lieber zur Schule gegangen sei, ob es sich durch die Schulbegleitung sicherer gefühlt habe und ob sich seine sozialen/kommunikativen Fähigkeiten, seine Konzentrationsfähigkeit, seine Impulskontrolle und seine Schulleistungen verbessert hätten. Die Zustimmungsquoten liegen, grob zusammengefasst, zwischen $50 \%$ (vermehrt bei „lieber zur Schule gegangen“ und Verbesserung der Impulskontrolle) und 75\% (vermehrt bei Gefühl von Sicherheit, Verbesserung sozialer/kommunikativer Fähigkeiten und Konzentrationsfähigkeit). Die Einschätzungen durch Schulbegleiter/innen, Lehrkräfte und Eltern weisen keine nennenswerten Differenzen auf und lassen keine durchgängigen Tendenzen je Personengruppe erkennen. Lediglich hinsichtlich der Verbesserung der Schulleistungen durch die Schulbegleitung äußern sich erkennbar weniger Lehrkräfte (50\%) als Schulbegleiter/innen und Eltern (63\% bzw. 69\%) zustimmend. Die Bewertungen der Schüler/innen entsprechen in diesen Punkten etwa denen der anderen Personengruppen, lediglich bei der Einschätzung 
des gesteigerten Sicherheitsgefühls und der Verbesserung der Impulskontrolle fallen sie leicht negativer aus. Die Autor/innen sehen in ihren Daten Hinweise auf einen Zusammenhang zwischen der Bewertung der Kooperation und der Bewertung der Maßnahme, nehmen aber keine inferenzstatistischen Analysen vor.

Die Ergebnisse von Böing (2013) zur Evaluation von Schulbegleitung für sehbeeinträchtige Schüler/innen stellen eine Zusammenfassung der Diskussion von betroffenen Schüler/innen, Lehrkräften, Schulbegleitungen, Schulleitungen und Vertreter/innen der Schulaufsicht dar. Dabei werden insbesondere das Fehlen klarer Vorgaben hinsichtlich der Qualifikation, der Aufgabenbereiche und der Einarbeitung von Schulbegleiter/innen kritisiert. Zu den geforderten notwendigen Qualifikationen zählen die Teilnehmenden der Diskussion auch die „fachliche Kompetenz in Bezug auf den Unterrichtsstoff“ (S. 17), „insbesondere in den verschiedenen Fächern der Sekundarstufe" (S. 18). Zudem wird die Reflexion und Klärung des Rollenverständnisses für wichtig erachtet, damit Schulbegleitung nicht zu einem Hindernis bei der sozialen Integration in die Klasse wird. Ähnliche Ergebnisse finden sich auch in der Auswertung der Gruppendiskussion von Schulbegleiter/innen, Lehrkräften und Eltern bei Deger et al. (2015). Demnach ist insbesondere das „Rollenverständnis der Schulbegleitung als Dolmetscher zwischen Kindern, Lehrkräften, Eltern und anderen Beteiligten“ (S. 167) von großer Bedeutung, ebenso auch eine schulübergreifende Konzeption von Schulbegleitung und eine gemeinsame Zielvorstellung aller Beteiligten. Auch die Diskussionsteilnehmer/innen bei Lindmeier et al. (2014) fordern eine stärkere Einbindung der Schulbegleitung in ein ,multiprofessionelles Team“ (S. 27). Das Thema Rollenverständnis greifen Lübeck \& Heinrich (2016) in ihrer Fallrekonstruktion auf, indem sie analysieren, wie die von ihnen interviewte Schulbegleiter/in ihre für sie selbst ungeklärte Rolle in der Beziehung zu den jeweiligen Lehrkräften zu vereindeutigen versucht. Als ein Hindernis wird dabei ihre fehlende professionelle Reflexionsfähigkeit identifiziert, die es erschwert, ein professionelles Arbeitsverhältnis jenseits von Vergemeinschaftung oder Konkurrenz aufzubauen.

\subsubsection{Internationale Befunde}

Auch in anderen Ländern wird nicht einschlägig qualifiziertes Assistenzpersonal in der Schule eingesetzt, insbesondere im gemeinsamen Unterricht von Schüler/innen mit und ohne sonderpädagogischen Unterstützungsbedarf (special needs). Begrifflich werden sie überwiegend als paraprofessionals (USA) oder teacher assistants (Kanada) bzw. teaching assistants (England) zusammengefasst. Der besseren Lesbarkeit halber verwende ich bezogen auf die internationale Forschung den Begriff Assistenzkerätte für Personal, das im Unterrichtskontext eingesetzt wird, ohne eine pädagogische Ausbildung absolviert zu haben. (Die Bezeichnung Schulbegleitung verwende ich weiterhin nur für Schulbegleiter/innen in Deutschland.) Die verfügbaren Studien zu schulischem Assistenzpersonal stammen überwiegend aus dem englischsprachigen Raum. Neben Überblicksartikeln über bisherige Forschung (Chambers, 2015; Farrell, Alborz, Howes \& Pearson, 2010; Giangreco, 2013; Giangreco, Halvorsen, Doyle \& Broer, 2004; Giangreco, Suter \& Doyle, 2010; Hanover Research, 2010) umfasst die Darstellung des internationalen Forschungsstandes eine Reihe kleinere Studien bzw. Studien, in denen Assistenzpersonal nur ein Punkt von mehreren ist (Azad, Locke, Downey, Xie \& Mandell, 2015; Butt \& Lowe, 2012; Giangreco \& Broer, 2005; Köpfer, 2013a, 2013b; Stivers \& Cramer, 2015; Takala, 2007), von denen aber ein Gewinn für die Untersuchung der Kooperation mit Schulbegleitung erwartet werden kann. Außerdem werden Befunde einer österreichischen Befragung von Schulassistent/innen (Bacher, Pfaffenberger \& Pöschko, 2007) einbezogen und die einer Längsschnittstudie aus England, die die bislang umfangreichste Studie zu Einsatz und Wirkung von Assistenzpersonal darstellt (Blatchford et al., 2009a, 2009b; Webster et al., 2010, 2011). Die Darstellung erfolgt im Vergleich zur Darstellung der Schulbegleitungsforschung in gekürzter Form und konzentriert sich auf die folgenden Punkte:

- Qualifikation und Tätigkeitsfelder

- Kooperation und Rollenbestimmung

- Evaluation

Qualifikation und Tätigkeitsfelder. In vielen westlichen Ländern wird ein Anstieg von nicht professionellen Assistenzkräften in Schulen verzeichnet, die insbesondere bei der Beschulung von Schüler/innen mit besonderem Förderbedarf und oft in inklusiven Settings eingesetzt werden, z. B. in den USA, Australien, Kanada, Finnland, England und Österreich (siehe Azad et al., 2015; Bacher et al., 2007; Giangreco, 2013; Köpfer, 2013a, 2013b; Prammer-Semmler \& Prammer, 2014; Takala, 2007). Teilweise wird in der Literatur unterschieden zwischen klassenbezogenen Assistenzkräften und solchen, die nur für ein einzelnes Kind 
oder eine/n Jugendliche/n zuständig sind (one-to-one-assistant oder personal assistant). Letztere werden im Folgenden individuelle Assistenzkraft genannt. Während individuelle Assistenzkräfte etwa in Finnland nur selten eingesetzt werden (Takala, 2007), macht diese Gruppe in Oberösterreich fast die Hälfte (Bacher et al., 2007) und in den USA über die Hälfte (Azad et al., 2015; Hanover Research, 2010) der Assistenzkräfte in der Beschulung von Kindern und Jugendlichen mit Förderbedarf aus. Generell gilt vermutlich nicht nur für die USA, dass in diesem Bereich weitaus mehr Assistenzkräfte als sonderpädagogische Lehrkräfte arbeiten (Stivers \& Cramer, 2015)

Ebenso wie Schulbegleiter/innen benötigen Assistenzkräfte in der Regel keine formale Qualifikation, die einer Ausbildung oder einem Studium im pädagogischen Bereich nahekäme (Bacher et al., 2007; Butt \& Lowe, 2012; Webster et al., 2010), allenfalls wird ein Kurzlehrgang (z. B. in Finnland, Takala, 2007) vorausgesetzt oder optionale Qualifizierungsprogramme angeboten (Bacher et al., 2007; Giangreco et al., 2010). Bezüglich der Tätigkeitsfelder von Assistenzkräften ist die rechtliche Situation in Deutschland der in Oberösterreich am ähnlichsten: Auch dort sind die Assistenzkräfte nicht für Aufgaben vorgesehen, die zu den pädagogisch-unterrichtlichen Aufgaben von Lehrkräften gehören (Bacher et al., 2007; Prammer-Semmler \& Prammer, 2014). In den meisten anderen Ländern unterliegt der Tätigkeitsbereich von Assistenzkräften keiner solchen Beschränkung und umfasst neben Unterstützungsleistungen in der Pflege und dem Sozialverhalten von Schüler/innen auch offiziell die fachlich-didaktische Instruktion im Unterricht (Fisher \& Pleasants, 2012; Giangreco, 2013; Giangreco et al., 2010; Giangreco \& Broer, 2015; Takala, 2007), was sich auch in der Bezeichnung als teaching assistant niederschlägt. Verschiedene Untersuchungen zeigen, dass dabei zunehmend auch solche Aufgaben übernommen werden, die genuin in den Zuständigkeitsbereich ausgebildeter Lehrkräfte fallen, nämlich die eigenständige Planung und Durchführung von Unterrichtseinheiten (Azad et al., 2015; Giangreco, 2013; Giangreco et al., 2010; Köpfer, 2013a, 2013b; siehe auch Bacher et al., 2007, und Hanover Research, 2010), und dass insbesondere Schüler/innen mit besonderem Unterstützungsbedarf einen Großteil der Lerninstruktionen von Assistenzkräften erhalten (Blatchford et al., 2009b, 2009c; Giangreco, 2013).

Kooperation. Die Ergebnisse von Studien, in denen explizite Fragen zur Zusammenarbeit zwischen Assistenzkräften und Lehrkräften gestellt werden, fallen unterschiedlich aus. In einer oberösterreichischen Studie gab die Mehrheit der Assistenzkräfte (69\%) häufige Besprechungen mit der Klassenleitung an, außerdem gingen anspruchsvollere Aufgaben mit verstärkter Teamarbeit einher (Bacher et al., 2007). In der gleichen Studie fand sich ein Zusammenhang zwischen dem Kooperationsumfang und dem Umfang von Überstunden, was nahelegt, dass Zeiten für Besprechungen nicht in den Arbeitsumfang eingerechnet sind (ebd.; siehe auch Chambers, 2015). Auf dieses Problem weisen auch Blatchford et al. (2009b) hin. Ihren Ergebnissen nach fällt die Kooperation zwischen Lehrkräften und Assistenzkräften je nach Schulform unterschiedlich aus. Am besten schneiden Förderschulen ab. Auch dort ist aber nur bei etwa der Hälfte der Befragten Zeit für gemeinsame Besprechungen vorgesehen, in Grundschulen nur bei etwa einem Drittel und an Allgemeinen weiterführenden Schulen bei unter 10\% (ebd.). Wie auch Giangreco et al. (2010) konstatieren, ist insgesamt davon auszugehen, dass vielerorts ein Mangel an strukturierter Anleitung und Kooperation herrscht.

Spezieller wird die Kooperation zwischen Lehrkräften und Assistenzkräften häufig im Zusammenhang mit der Angemessenheit des Einsatzes von Assistenzkräften untersucht. Dabei wird gefragt, ob Assistenzkräfte ausreichend angeleitet und begleitet werden, Zuständigkeiten geklärt sind und ihr Aufgabenbereich ihrer Rolle und Qualifikation entspricht. Bei der Zusammenarbeit mit Assistenzkräften tragen in der Regel die Lehrkräfte die Verantwortung für die Anleitung und Supervision (Bacher et al., 2007; Stivers \& Cramer, 2015), wobei als Erschwernis die mangelnde Erfahrung und Ausbildung von Lehrkräften als Teamleitung genannt wird (Blatchford et al., 2009a, 2009b; Takala, 2007; Webster et al., 2010, 2011). Wie die Studie von Butt und Lowe (2012) zeigt, kann nicht vorausgesetzt werden, dass die Assistenzkräfte und die Lehrkräfte, mit denen sie zusammenarbeiten, über eine klare Vorstellung von den Aufgaben einer Assistenzkraft im sonderpädagogischen Bereich und den hierfür notwendigen Kompetenzen verfügen. Fehlende Rollenklarheit führt bei den Befragten zu einer Diffusion von Zuständigkeiten und erschwert effektive Kooperation und eine angemessene Arbeitsteilung (ebd.). Ähnliche Zusammenhänge werden auch in anderen Studien gefunden (Blatchford et al., 2009a, 2009b; Hanover Research, 2010; Köpfer, 2013a, 2013b; Webster et al., 2010, 2011). Verschiedene Untersuchungen zeigen zudem, dass Assistenzkräfte einen nicht unbedeutenden Teil ihrer Arbeitszeit im Klassenraum mit Nichtstun bzw. Warten (Takala, 2007) oder mit der Verrichtung 
persönlicher Angelegenheiten (z. B. Handynutzung) verbringen (Azad et al., 2015). Azad et al. (2015) vermuten, dass eine besser abgestimmte Zusammenarbeit mit der Lehrkraft einen effektiveren Einsatz ermöglichen würde.

Evaluation. Die Bedeutung der Art und Weise, wie Assistenzkräfte eingesetzt werden, wird nicht nur bei Effektivitätsfragen deutlich, sondern zeigt sich auch in den gemischten Befunden hinsichtlich der Wirkungen von Unterstützung durch Assistenzkräfte. Ihr Einsatz zeigt positive Effekte auf die Disziplin im Klassenraum und entlastende Effekte für Lehrkräfte, die sich in höherer Arbeitszufriedenheit sowie reduziertem Arbeitsstress äußern (Blatchford et al., 2009a, 2009b, 2009c; siehe auch Köpfer, 2013a, 2013b). Ein positiver Einfluss auf die Lernleistung von Schüler/innen zeigt sich jedoch nicht in jedem Fall, sondern offenbar vor allem dann, wenn die Assistenzkräfte spezifische Lernprogramme durchführen, für die sie zuvor geschult wurden (Farrell et al., 2010). Bedenkenswert sind zudem Befunde, nach denen individuelle Assistenz auch zu unnötiger Abhängigkeit, Stigmatisierung des betreffenden Schülers oder der Schülerin, Erschwerung von Peer-Interaktionen und geringerer Lernunterstützung durch die Lehrkraft führen kann (Giangreco \& Broer, 2005; Giangreco et al., 2004, 2010; Köpfer, 2013a, 2013b).

Wie die bislang umfangreichste Studie zur Wirkung von Assistenzkräften (Blatchford et al., 2009a, 2009b; Webster et al., 2010, 2011) zeigt, ist eine geringere Lernunterstützung durch die Lehrkraft als unerwünschter Nebeneffekt von Assistenzeinsatz nicht zu unterschätzen. Über einen Zeitraum von fünf Jahren untersuchte die Forschergruppe die Effekte von Assistenzkräften auf die Lernleistung von Schüler/innen in Mathe, Englisch und den Naturwissenschaften. Dabei fanden sie, dass Schüler/innen, die mehr Unterstützung durch Assistenzkräfte erhielten, geringere Lernfortschritte machten als vergleichbare Schüler/innen, die weniger Unterstützung durch Assistenzkräfte erhielten. Die Autor/innen vermuten, dass der Grund für diesen kontraintuitiven Effekt in der teilweisen Ersetzung der (professionellen) Lehrerinstruktion durch die Assistenzkräfte liegt: Es zeigte sich, dass die Assistenzkräfte die meiste Zeit auf individueller oder Gruppenbasis mit leistungsschwächeren Schüler/innen arbeiteten, die hierfür teilweise vom Klassenverband getrennt wurden. Für diese Aufgaben wurden die Assistenzkräfte ungenügend oder gar nicht vorbereitet, es mangelte etwa, wie bereits ausgeführt, an Besprechungszeiten mit den Lehrkräften (Blatchford et al., 2009a, 2009b). Beobachtungen der Arbeitsweise von Lehrkräften und Assistenzkräften offenbarten unterschiedliche didaktische Vorgehensweisen, die Hinweise darauf liefern, weshalb Schüler/innen mit mehr Assistenzunterstützung weniger Lernfortschritte machen: Während Lehrkräfte häufiger offene Fragen stellten, mehr Feedback gaben und mehr Zeit für Erklärungen aufwandten, neigten Assistenzkräfte stärker dazu, auf die Beendigung von Aufgaben zu fokussieren und Antworten vorwegzunehmen. Aufbauend auf diesen Ergebnissen wurden Richtlinien zum effektiven Einsatz von Assistenzkräften entwickelt (Webster, Russell \& Blatchford, 2013b), deren Umsetzung bereits zu positiv evaluierten Veränderungen führte (Blatchford, Webster \& Russell, 2012). Hilfreich ist demnach, die Rolle von Assistenzkräften in einem schuleigenen Konzept einheitlich zu definieren, Supervision und Fortbildungen zur Qualifizierung von Assistenzkräften sicherzustellen und regelmäßige Besprechungszeiten mit den Lehrkräften, in deren Unterricht sie eingesetzt sind, zu implementieren. Ein Vorteil gegenüber Qualifizierungsprogrammen, die sich nur an Assistenzkräfte wenden, besteht darin, dass auf diese Weise wichtige Rahmenbedingungen verändert werden können, auf die Assistenzkräfte selbst keinen Einfluss haben.

Ein Teil der Probleme, die im Zusammenhang mit dem Einsatz von Assistenzkräften formuliert werden, besteht auch aus Sicht der Assistenzkräfte selbst. Hierzu zählen z. B. die fehlende Rollenklarheit (Butt \& Lowe, 2012), die Sorge um unnötige Abhängigkeit der Schüler/innen (Giangreco \& Broer, 2005) und Zweifel, ob die eigene Expertise für die erfolgreiche Bewältigung der Aufgaben ausreicht (Bacher et al., 2007; Fisher \& Pleasants, 2012). Unzufrieden zeigen sich die Assistenzkräfte mit der geringen Entlohnung ihrer Arbeit (Bacher et al., 2007; Blatchford et al., 2009b) und nach Fisher und Pleasants (2012) auch mit der geringen Wertschätzung. Üblich scheinen befristete Teilzeitverträge und ein geringer Lohn (Bacher et al., 2007; Chambers, 2015; Takala, 2007). Bemerkenswerterweise werden dennoch positive Gesamtzufriedenheitswerte gefunden (Bacher et al., 2007; Blatchford et al., 2009b). Dabei deuten die Ergebnisse von Bacher et al. (2007) darauf hin, dass sowohl stärkere Kooperation als auch die Übernahme von pädagogischen Tätigkeiten die Arbeitszufriedenheit erhöht (Bacher et al., 2007; siehe auch Köpfer, 2013a, 2013b). 


\subsection{Zusammenfassung}

Zu Beginn dieses Kapitels wurden die rechtlichen Grundlagen von Schulbegleitung als Eingliederungshilfe erläutert und der Zusammenhang von schulgesetzlichen Änderungen, die die verstärkte Inklusion von Schüler/innen mit sonderpädagogischem Förderbedarf zum Ziel haben, mit dem Anstieg von Schulbegleitungen diskutiert. Die anschließende Darstellung der Bewilligungs- und Durchführungspraxis in Stadt und Landkreis Göttingen hat verdeutlicht, dass im Bereich Schulbegleitung neben den Schulbegleiter/innen selbst eine Vielzahl von Personen beschäftigt ist, um die Bewilligungsvoraussetzungen zu prüfen und die Durchführung der Maßnahme zu begleiten. Eine mögliche Besonderheit in Göttingen ist, dass dieses Personal nicht nur die üblichen Mitarbeiter/innen der Ämter und der Träger umfasst, sondern in den Jugendämtern spezielle Koordinatorenstellen geschaffen wurden und ein großer Teil der Maßnahmenprüfung und -begleitung durch eine spezielle Fachstelle erfolgt. Insgesamt scheint, dass von den Jugendämtern feste personelle und konzeptuelle Strukturen im Bereich Schulbegleitung entwickelt wurden. Dass die Fachstelle ausschließlich für die Jugendämter arbeitet, zeigt die Unterschiedlichkeit der Arbeitsweisen der Jugend- und Sozialämter im Bereich Schulbegleitung.

Die Schwierigkeiten, die insbesondere mit der Ausweitung der Maßnahme Schulbegleitung im inklusiven Unterricht verbunden sind, wurden anhand zentraler Kritikpunkte aktueller Diskussionen um Schulbegleitung deutlich, die sich vor allem auf die Frage des angemessenen Einsatzes von Schulbegleitung beziehen (z. B. AGJ, 2014; Böing, 2013; Dworschak, 2010; Knuf, 2013; Lübeck, 2016a). Wie in der Darstellung des Forschungsstandes sichtbar wurde, liegen den Diskussionen nur wenige empirische Befunde zugrunde. Die meisten der insgesamt wenigen Studien zu Schulbegleitung beschränken sich auf Abfragen zur Verteilung von Schulbegleitungen auf Schulformen und Schülermerkmale sowie die Qualifikation und die Aufgabenbereiche von Schulbegleiter/innen. Im Vergleich mit der Verteilung der Gesamtschülerzahlen auf die unterschiedlichen Schulformen zeigt sich, dass an Grund- und Förderschulen überproportional häufig Schulbegleitung in Anspruch genommen wird (Deger et al, 2015; Wohlgemuth, 2009). Eine weitere wichtige Erkenntnis aus den verfügbaren Befunden ist, dass, obwohl die Tätigkeit an keine formale Qualifikation gebunden ist, ein nicht geringer Anteil von Schulbegleiter/innen einschlägig qualifiziert ist (Beck et al., 2010; Dworschak 2012c; Henn et al., 2014; Wohlgemuth, 2009). Nur wenige Studien beschäftigen sich mit der Evaluation der Maßnahme, indem sie die Beteiligten nach ihrer Einschätzung fragen (Dworschak, 2012b, 2012c; Wohlgemuth, 2009; Zauner \& Zwosta, 2014). Trotz der dünnen Studienlage wird deutlich, dass die gesetzlich vorgesehene Trennung schulischer Aufgaben von Schulbegleitertätigkeiten in der Praxis nicht eingehalten wird (Beck et al., 2010; Dworschak, 2012b, 2012c; Lindmeier et al., 2014; Wohlgemuth, 2009). Obwohl die Unklarheit der Zuständigkeiten und Rollen als Problem gesehen wird, wie insbesondere qualitative Studien zeigen (Böing, 2013; Heinrich \& Lübeck, 2013; Lindmeier et al., 2014; Lübeck \& Heinrich, 2016), scheint die Mehrheit der Schulbegleiter/innen sowie der beteiligten Lehrkräfte, Eltern und auch Schüler/innen insgesamt zufrieden mit der Maßnahme zu sein (Dworschak, 2012b, 2012c; Wohlgemuth, 2009; Zauner \& Zwosta, 2014).

Differenzierte Untersuchungen zum Einsatz von nichtprofessionellem Assistenzpersonal im inklusiven Unterricht liegen aus England, Finnland, Kanada, Australien, Österreich und den USA vor. Die Befunde zeigen, dass die Passung von Aufgabenbereichen und Qualifikation der Assistenzkräfte häufig nicht gegeben ist und die jeweiligen Zuständigkeiten von Lehrkraft und Assistenzkraft nicht eindeutig geklärt sind (Butt \& Lowe, 2012; Hanover Research, 2010; Köpfer 2013a, 2013b; Webster et al., 2010, 2011). Insbesondere die Ergebnisse der breit angelegten Längsschnittstudien von Blatchford et al. (2009a, 2009b), machen deutlich, dass unter diesen Bedingungen sogar kontraproduktive Effekte vom Assistenzeinsatz im Unterricht ausgehen können. Obwohl der Einsatz und die Tätigkeitsfelder von Assistenzkräften in anderen Ländern auf anderen rechtlichen Grundlagen fußen und anders ausgeformt sind, finden sich viele Parallelen zu Schulbegleiter/innen: Zwar dürfen Schulbegleiter/innen keine unterrichtlich-pädagogischen Tätigkeiten übernehmen; empirische Studien zeigen aber, dass sie dies durchaus tun. Damit ergibt sich für beide Gruppen eine größere Überschneidungsmenge im Aufgabenbereich als von der Konzeption her angelegt. Auch hinsichtlich der Arbeitsbedingungen und der Unklarheit ihres spezifischen Auftrags scheinen Schulbegleitungen und Assistenzkräfte vergleichbar. Insgesamt zeigt sich, dass die Fragen, die im internationalen Kontext empirisch untersucht werden, einem Teil der Fragen entsprechen, die in der Diskussion um Schulbegleitung theoretisch erörtert werden (z. B. von Lübeck \& Heinrich, 2016). Zu nennen ist hierbei insbesondere die Frage der Gestaltung von Assistenzeinsatz mit Hinblick auf mögliche negative Auswirkungen im Kontext von Inklusion. Im Diskussionsteil dieser Arbeit werde ich auf diese Fragen zurückkommen. 


\section{Kooperation im Bereich Schule}

Im Folgenden werden, wie in der Forschungsliteratur üblich, unter schulischer Kooperation jegliche Formen von Zusammenarbeit von Personen gefasst, die beruflich oder ehrenamtlich an der Schule tätig sind. Zur theoretischen Einordnung schulischer Kooperation werden zunächst Definitionsansätze von Kooperation vorgestellt und anschließend die für Schule charakteristischen Rahmenbedingungen für Kooperation beschrieben sowie aktuelle Wandlungsprozesse im Bereich schulischer Kooperation. Daraufhin erfolgt eine Diskussion der Bedeutung, die Kooperation als Schulqualitätsmerkmal zugeschrieben wird, und der Besonderheiten multiprofessioneller Kooperation. Zuletzt werden theoretische Modelle schulischer Kooperation vorgestellt.

Der empirische Forschungsstand wird bezogen auf Lehrerkooperation und bezogen auf multiprofessionelle Kooperation aufgearbeitet. Obwohl die vorliegende Studie multiprofessionelle Kooperation untersucht, wird der Lehrerkooperation aufgrund ihrer elaborierteren theoretischen Ausarbeitung und der breiten empirischen Befundlage ein eigenes Unterkapitel gewidmet. Zusätzlich begründet ist dies durch die Zielsetzung dieser Arbeit, Erkenntnisse für das kooperative Handeln von Lehrkräften zu gewinnen. Zu Lehrerkooperation werden insbesondere Studien vorgestellt, die auf Grundlage der zuvor beschriebenen theoretischen Modelle entwickelt wurden. Empirischen Befunden zum Lehrerkooperationsmodell von Fussangel (2008) wird weiter Raum eingeräumt, da von diesem Modell das Potential zur grundlegenden Konzeption der Zusammenarbeit von Lehrkräften mit anderen Berufsgruppen und damit für diese Untersuchung erwartet wird. Die Darstellung der empirischen Befunde erfolgt in inhaltlicher Gruppierung von Inhalt und Ausmaß sowie Zielen und Nutzen von Kooperation. Es wird der inhaltlichen Gruppierung also der Vorzug gegenüber einer kompakten Darstellung von Theorie und Überprüfung des Modells von Fussangel (2008) gegeben, um die Ergebnisse mit ähnlichen oder darauf aufbauenden Ergebnissen anderer Studien zu verknüpfen.

$\mathrm{Zu}$ multiprofessioneller Kooperation werden Befunde zur Kooperation von Lehrkräften mit an der Schule pädagogisch tätigen Nicht-Lehrkräften und mit Sonderpädagog/innen dargestellt. Begrifflich fallen Sonderpädagog/innen zwar unter Lehrerkooperation, im Kontext dieser Arbeit sind aber vor allem die Aspekte der Zusammenarbeit interessant, die mit der Zugehörigkeit beider Lehrergruppen zu verschiedenen Professionen verbunden sind, weswegen ihre Darstellung im Unterkapitel zu multiprofessioneller Kooperation erfolgt. Abschließend werden die theoretischen Ausführungen und die berichteten empirischen Befunde gebündelt zusammengefasst. 


\subsection{Theoretische Einordnung}

Zur theoretischen Einordnung schulischer Kooperation wird im Folgenden zunächst der Begriff der Kooperation anhand von Definitionsansätzen verschiedener Disziplinen näher bestimmt. Auf die Besonderheiten von Kooperation im Schulkontext wird mit der Erläuterung der Rahmenbedingungen, die die professionelle Arbeit in der Schule prägen, eingegangen, von denen angenommen wird, dass sie Kooperation eher erschweren als fördern. In Zusammenhang damit werden verschiedene jüngere Entwicklungen beschrieben, die zu einem erheblichen Anstieg von Kooperationsanlässen in Schulen geführt haben. AnschlieBend werden die positiven Erwartungen, die an Kooperation gestellt werden, theoretisch diskutiert und die Besonderheiten multiprofessioneller Kooperation erläutert. Abschließend werden Modelle zur Kategorisierung von Formen schulischer Kooperation vorgestellt.

\subsubsection{Begriffsbestimmung}

Kooperation ist Gegenstand verschiedener Wissenschaftsdisziplinen, die sich mit menschlicher Interaktion beschäftigen. Eine allgemeine Definition findet sich bei Argyle (1991, S. 4): „Cooperation: acting together, in a coordinated way at work, leisure, or in social relationships, in the pursuit of shared goals, the enjoyment of the joint activity, or simply furthering the relationship“. Aus sozialpsychologischer Perspektive wird Kooperation als charakteristisches Merkmal des Menschen als sozialem Wesen gesehen und erfüllt in bestimmten Kontexten keine weiteren Funktionen als ein positives Gemeinschaftserleben oder die Stärkung der Beziehung zwischen den Kooperationspartner/innen.

In der sozialwissenschaftlichen Literatur wird der Begriff Kooperation meist im Sinne von Arbeitskooperation benutzt, um Tätigkeiten der Zusammenarbeit im beruflichen Kontext zu beschreiben. Auch in dieser eingegrenzten Verwendung schließt Kooperation noch ein breites Spektrum verschiedener Handlungen ein und entsprechend ist es nicht verwunderlich, dass eine unzureichende Spezifizierung der Begriffsverwendung in der Forschungsliteratur beklagt wird (z. B. Fussangel, 2008; Schneider, 2004; Spieß, 2004). An dieser Stelle erfolgt daher zunächst die Darstellung allgemeiner Definitionsmerkmale von Kooperation aus der Perspektive von für diese Arbeit relevanten Bezugswissenschaften. Vorab sei bemerkt, dass allgemeine Definitionsansätze nicht zwischen intraprofessioneller Kooperation und interprofessioneller bzw. multiprofessioneller Kooperation ${ }^{16}$ unterscheiden, sondern auf beide Formen anwendbar sind.

Kurz gesagt bedeutet Kooperation im beruflichen Kontext „Zusammenarbeit von zwei oder mehr Personen“ (Esslinger, 2002, S. 62, im Original jeweils kursiv) oder „Abstimmung der Beteiligten“ (van Santen \& Seckinger, 2003, S. 29, im Original jeweils kursiv). Voraussetzungen für die Möglichkeit zur Kooperation ist nach Esslinger (2002, S. 62) „ein vorhandenes Potential zur strukturellen und funktionalen Anbindung der Arbeitsfelder“. Gemeinsam ist diesen beiden Definitionsansätzen aus der Sozial- und Bildungsforschung, dass durch Kooperation ein gemeinsames Ziel erreicht werden soll, nämlich „eine Optimierung von Handlungsabläufen oder eine Erhöhung der Handlungsfähigkeit bzw. Problemlösekompetenz" (van Santen \& Seckinger, 2003, S. 29) bzw. „die Effektivität der Arbeit und die Zufriedenheit mit der Arbeit zu steigern“ (Esslinger, 2002, S. 62). Nach Spieß (2004) kann Kooperation sowohl zu Entlastung durch Arbeitsteilung als auch zu Belastung durch den Abstimmungsaufwand führen. Auf ihrer arbeitspsychologischen Definition bauen einschlägige bildungswissenschaftliche Modelle und Studien auf (z. B. Dizinger, 2015; Fussangel, 2008; Gräsel et al., 2006): „Kooperation ist gekennzeichnet durch den Bezug auf andere, auf gemeinsam zu erreichende Ziele bzw. Aufgaben, sie ist intentional, kommunikativ und bedarf des Vertrauens. Sie setzt eine gewisse Autonomie der Akteure voraus und ist der Norm der Reziprozität verpflichtet" (Spieß, 2004, S. 199). Auch in dieser Definition spielt das gemeinsame Ziel eine Rolle, bleibt aber ohne inhaltliche Bestimmung. Bei der Kooperation innerhalb von Organisationen kann an der Stelle des gemeinsamen Zieles auch der Versuch der gemeinsamen Aufgabenbewältigung stehen, für den die Organisation die formalen Rahmenbedingungen setzt (Spieß, 2004). In diesem Fall ist nicht nur Vertrauen in den Kooperationspartner/innen, sondern auch ,in die formalen Abläufe“ (ebd., S. 211) wichtig. Dabei bewirkt Kooperation einerseits eine „Ausweitung der Entscheidungs- und Handlungsspielräume“ und „zugleich auch deren Einengung" (ebd.).

Die Verbindung von Kooperation und Organisation führen Kuper und Kapelle (2012) aus organisations- und professionstheoretischer Perspektive näher aus, indem sie Kooperation als „Zwischenschritt zur

16 Für die Termini interprofessionelle Kooperation und multiprofessionelle Kooperation findet sich keine sinnvolle definitorische Abgrenzung; sie werden in dieser Arbeit synonym verwendet. 
Organisation" (S. 41) bezeichnen und die Rolle der formalen Organisation zur Stabilisierung und Verstetigung von Kooperation betonen. Sie gehen davon aus, dass gemeinsame Ziele unter den Mitgliedern einer Organisation nicht per se vorhanden sein müssen und zu ihrer Herstellung ein formaler Rahmen vonnöten ist. Die Aushandlung von Zielen ist dieser Sichtweise nach der Prozess, durch den Kooperation zur Professionalisierung beiträgt: „Mit der Kooperation in der professionellen Gemeinschaft sind ein kommunikatives Format und ein sozialer Ort gekennzeichnet, in dem die Verständigung über die theoretischen, praktischen und moralischen Aspekte der professionellen Tätigkeit erfolgt. [...] Die Professionellen bilden eine ,Community of Practice', in der sie das ihnen exklusiv zugängliche Wissen konstruieren und im Interesse der Verbesserung professioneller Praxis kommunizieren“" (ebd., S. 44). ${ }^{17}$

Zusammenfassend lässt sich professionelle Kooperation als Prozess verstehen, in dem durch kommunikative Bezugnahme aufeinander gemeinsame Ziele ausgehandelt oder erreicht werden sollen oder Aufgaben gemeinsam bearbeitet werden. Voraussetzungen hierfür sind Intentionalität, Vertrauen und eine gewisse Autonomie bei gleichzeitiger gegenseitiger Abhängigkeit. Kooperation kann den eigenen Handlungsspielraum sowohl eingrenzen als auch ausweiten und sowohl entlasten als auch belasten. Im kooperativen Handeln kann sich professionelle Praxis weiterentwickeln. Theoretisch hiervon abgrenzbar ist Kooperation, die unabhängig von den Aufgaben oder Zielen, die der professionelle Kontext oder der organisationale Rahmen vorgibt, stattfindet, um durch zwischenmenschliche Interaktion Wohlbefinden zu erleben oder Bindungen zu schaffen. Beide Formen von Kooperation können in beruflichen Kontexten stattfinden und ineinandergreifen.

\subsubsection{Rahmenbedingungen und Besonderheiten schulischer Kooperation}

Schule gilt traditionell als eine monoprofessionelle Institution, die von Rahmenbedingungen geprägt ist, die kooperatives Handeln erschweren. Einflussreiche Analysen zur Organisationskultur von Schule und zur beruflichen Sozialisation von Lehrkräften lieferten Weick (1976) und Lortie (1975). Demnach lassen sich Bildungseinrichtungen als loosely coupled systems beschreiben (Weick, 1976): Die einzelnen Organisationselemente sind nur lose miteinander verbunden und agieren relativ autonom. Entsprechend charakterisiert eine hohe Unabhängigkeit das berufliche Handeln von Lehrkräften. Nach Lortie (1975) ist die Berufskultur an Schulen vom Autonomie-Paritäts-Muster geprägt: Durch den Umstand, dass Lehrkräfte ihre Kerntätigkeit, das Unterrichten von Klassen, isoliert von Kolleg/innen ausüben und dabei über eine hohe Gestaltungsfreiheit verfügen, habe sich die Norm der Nichteinmischung in die Arbeitsangelegenheiten von Kolleg/innen entwickelt (siehe auch Terhart, 1991). Die relativ hohe Autonomie der schulischen Teileinheiten und der Lehrtätigkeit sind nicht als grundsätzlicher Nachteil für die Bewältigung schulischer Anforderungen zu sehen (vgl. Kuper \& Kapelle, 2012), sie wird aber oftmals als Kooperationshindernis gedeutet (z. B. Rolff, 1980; Ulich, 1996). Auf organisationaler Ebene sind engere Kooperationsstrukturen in der Regel schwach ausgeprägt und auf personaler Ebene kann der Mangel an kollegialer Rückmeldung über die Qualität der eigenen Arbeit zu einer Unsicherheit führen, die Kooperation aus Angst vor Bewertung meidet (Altrichter, 2000; Terhart, 1991). Aus professionstheoretischer Perspektive fehlt es innerhalb einer solchen Organisationsstruktur an Anlässen bzw. an der Notwendigkeit von kooperativem Handeln, das über bloße Koordinierungsleistungen hinausgeht (vgl. Kuper \& Kapelle, 2012). Erst die Notwendigkeit der kooperativen „Verständigung über die theoretischen, praktischen und moralischen Aspekte der professionellen Tätigkeit“ (ebd., 2012, S. 44) und der Aushandlung von gemeinsamen Zielen führen zu einer „Verbesserung professioneller Praxis“" (ebd.).

Die Entwicklungen der letzten Jahrzehnte haben in Schulen zu einer deutlichen Erhöhung des Kooperationsbedarfs geführt, einerseits innerhalb der Lehrerschaft und anderseits zwischen der Lehrerschaft und Vertreter/innen anderer Professionen und Berufsgruppen (vgl. Boller, 2012). Dazu beigetragen hat unter anderem die Stärkung der Autonomie von Einzelschulen, die einen Aufschwung lokaler Schulentwicklung bewirkt hat. Auf Einzelschulebene arbeiten Lehrkräfte nun vermehrt in sogenannten Steuergruppen zusammen oder bilden anlassgebundene Projektteams (Bauer, 2008; Fussangel, 2008). Auch die Einführung von Bildungsstandards als Reaktion auf die Ergebnisse der großen internationalen Vergleichsstudien von Schulleistungen führt etwa durch die Notwendigkeit engerer Abstimmung bezüglich der Unterrichtsinhalte zu

17 Ob Kooperation im Arbeitskontext immer als professionelle bzw. multiprofessionelle oder „,nur“ als berufliche Kooperation gelten kann, ist vom jeweiligen Professionsverständnis abhängig (siehe Merten \& Olk, 1999). Ob der Lehrerberuf eine Profession darstellt oder nicht, ist Gegenstand soziologischer Analyse (z. B. Rothland, 2009; Terhart, 1991). In der vorliegenden Arbeit spielt die Unterscheidung zwischen Profession, Beruf und ausgeübter/entlohnter Tätigkeit keine Rolle. 
mehr Kooperation zwischen Lehrkräften (Boller, 2012). Im Zusammenhang mit den PISA-Ergebnissen steht zudem der Ausbau von Ganztagsschulen, die eine bessere Förderung insbesondere bildungsbenachteiligter Schüler/innen leisten sollen und dem gestiegenen Anspruch an individuelle und ganzheitliche Bildung besser gerecht werden sollen (Böttcher et al., 2011). Dieses „erweiterte Bildungsverständnis“ (ebd., S. 103) sieht neben der klassischen Schulbildung auch die Vermittlung kultureller, sozialer und lebenspraktischer Kompetenzen vor, für die Ganztagsschulen entsprechende außerunterrichtliche Angebote bereithalten (siehe z. B. Appel, Ludwig, Rother \& Rutz, 2003; Böttcher et al., 2011). Neben der Organisation des Ganztagsbetriebs stellt auch die Einbindung des zusätzlich beschäftigten Personals für die Ganztagsangebote neue Anforderungen an die innerschulische Kooperation. Zuletzt wurden bedeutende Veränderungen im schulischen Alltagsgeschäft auch durch die Entwicklung im Bereich Inklusion bewirkt. Mit der Inklusion von Kindern und Jugendlichen mit Behinderung wird an Schule ein Integrationsanspruch gestellt, der die Zusammenarbeit insbesondere von Regellehrkräften und sonderpädagogischen Lehrkräften erfordert. Neben Sonderpädagog/innen wird im Regelschulbereich auf Unterrichtsebene vermehrt mit Erzieher/innen (Breuer, 2015) und mit Schulbegleiter/innen (Dworschak, 2012b; Henn et al., 2014; Kißgen et al., 2013) zusammengearbeitet. Insbesondere Kooperation innerhalb des Unterrichtsgeschehens selbst - dem Kerngeschäft von Schule - stellt für viele Lehrkräfte eine Neuerung dar (vgl. Fussangel, 2008). Die erhöhten Kooperationserfordernisse bedeuten Veränderungen sowohl auf organisationaler als auch auf personaler Ebene (Kuper \& Kapelle, 2012). Für die Schaffung kooperativer Rahmenbedingungen wird die Rolle der Schulleitung als zentral angesehen (z. B. Bauer, 2008); es wird davon ausgegangen, dass auch innerhalb der Rahmenbedingungen, die Schule als lose gekoppeltes System (Weick, 1976) charakterisieren, Spielraum für die Entwicklung kooperativer Strukturen vorhanden ist (Altrichter, 2000; Kuper \& Kapelle, 2012). Bei den Beteiligten selbst wird die Überwindung des „Lehrerindividualismus“ (Terhart, 1999, S. 463) für nötig gehalten. Was diese Veränderungen für die Professionalisierung von Lehrkräften und für schulische Innovationsprozesse bedeuten, ist Gegenstand des nächsten Abschnitts.

Kooperation, Professionalisierung und Innovation. Die beschriebenen Veränderungen im Schulsystem lassen aufgrund der erhöhten Kooperationsnotwendigkeit positive Effekte auf die Professionalisierung von Lehrkräften und auf Innovationsprozesse an Schulen erwarten. Nicht nur das deutsche Schulsystem ist im Wandel begriffen, so dass vergleichbare Entwicklungen auch in der internationalen Forschung untersucht und begleitet werden (für einen Überblick siehe Villegas-Reimers, 2003). Professionalisierung wird dabei nicht normativ im Sinne einer Abwertung des Ist-Zustandes an beruflicher Handlungskompetenz verstanden, sondern als Erweiterung vorhandenen Wissens, vorhandener Kompetenzen und Perspektiven sowie als Prozess der Weiterentwicklung oder Neubestimmung des eigenen Rollenverständnisses (vgl. ebd.). Diese beiden Aspekte von Professionalisierung - Weiterentwicklung der professionellen Praxis und Weiterentwicklung des Professionsverständnisses - sind eng miteinander verknüpft; in der bildungswissenschaftlichen Fachliteratur wird meist der Aspekt der Weiterentwicklung der professionellen Praxis fokussiert. Zur theoretischen Auseinandersetzung mit dem Zusammenhang von Kooperation, Professionalisierung und Innovation lohnt eine separate Betrachtung in Anlehnung an Kuper und Kapelle (2012).

Die Öffnung für kooperatives Arbeiten bedeutet für die Lehrerschaft nicht nur die Herausforderung, das eigene berufliche Handeln zu deprivatisieren, sondern bringt es mit sich, dass durch die Verhandlung von Kooperationszielen auch eine Verständigung über die professionelle Rolle nötig wird (vgl. Altrichter, 2000). Man kann davon ausgehen, dass sowohl berufliche Handlungsziele als auch die Lehrerrolle in der Schulpraxis wenig einheitlich und zudem wenig konkret gefasst sind (vgl. Fussangel, 2008; Fussangel \& Gräsel, 2012; Rothland, 2009; Terhart, 1991). ${ }^{18}$ Erschwert wird die Definition der Lehrerrolle auch durch die Komplexität und teilweise Widersprüchlichkeit der Ansprüche, die von verschiedenen Personengruppen (Schüler/innen, Lehrer/innen, Eltern, Vorgesetzte, Politik, Öffentlichkeit) an Lehrkräfte gestellt werden (Barth, 1997; Rothland, 2009; Schaarschmidt, 2006) und der Veränderung dieser Ansprüche - des „Mandats des Lehrerberufs" (Terhart, 1991, S. 467) - in Abhängigkeit von gesellschaftlichen Entwicklungen (Terhart, 1991; Wiater, 2009). Ein Beispiel ist die, auch durch die Fachwissenschaft getragene, Entwicklung vom lehrerzentrierten Frontalunterricht hin zum individualisierten, selbstregulierten Lernen, durch das die Lehrkraft vom „Hauptdarsteller zum Prozessbegleiter“ (Scholz, 2012, S. 39) wird. Für die Entwicklung eines von anderen Professionen abgrenzbaren Selbstverständnisses und die Entwicklung professioneller Standards ist

18 Ein einheitliches Professionsverständnis, wie es bei den klassischen Professionen Arzt, Jurist, Geistlicher vorhanden ist, kann nach Einschätzung verschiedener Autor/innen innerhalb der Lehrerschaft nicht vorausgesetzt werden (Rothland, 2009; Terhart, 1991). 
der Diskurs innerhalb der eigenen „Community of Practice“ (Kuper \& Kapelle, 2012; siehe auch Bondorf, 2013) zentral. Die durch Kooperation entstehenden Verständigungs- und Aushandlungsprozesse bergen daher das Potential, das Professionsverständnis des Lehrers oder der Lehrerin näher zu bestimmen und weiterzuentwickeln. Nicht nur durch den Diskurs innerhalb der eigenen Profession, auch von der Kooperation mit anderen Professionen können Impulse ausgehen, die die eigene Rolle durch Abgrenzungsprozesse näher bestimmen. Allerdings kann auch der gegenteilige Effekt einer Deprofessionalisierung durch Verwischung der Grenzen zwischen den Professionen eintreten, zumal die Lehrerrolle aufgrund ihrer Komplexität sehr unterschiedliche Anknüpfungspunkte bietet. Mit Fokus auf den schulischen Erziehungsauftrag kann sich eine Lehrkraft mit dem Professionsverständnis - und den beruflichen Handlungszielen - der Sozialpädagogik identifizieren, mit Fokus auf ihre Rolle als Fachkraft für Unterricht davon abgrenzen. Gerade die gestiegenen Ansprüche an eine umfassende Bildung und Erziehung der Schülerschaft lassen eine Eingrenzung der Lehrerrolle schwierig erscheinen, machen sie aber nicht weniger notwendig, wenn trotz dieser Komplexität professionelle Standards bestimmt werden sollen, die in der Praxis umsetzbar sind und in der multiprofessionellen Kooperation Orientierung bieten. Nicht zuletzt wird Klarheit über die eigene Berufsrolle und ihre Grenzen auch in der Arbeitsgesundheitsforschung für wichtig erachtet (Barth, 1997).

Parallel zur Bestimmung oder Weiterentwicklung des Professionsverständnisses dient der professionelle Diskurs der Weiterentwicklung der professionellen Praxis. In der Fachliteratur wird dies unter dem Stichwort Professionalisierung durch Kooperation diskutiert (z. B. Bondorf, 2013; Terhart, 1991) und in der Praxis mit dem Konzept der Professionellen Lerngemeinschaften (siehe hierzu z. B. Bonsen \& Rolff, 2006) umgesetzt. Auch Ansätze des situierten Lernens von Lehrkräften (z. B. Putnam \& Borko, 2000) gehen von einer Kompetenzerweiterung durch professionellen Diskurs aus. Putnam und Borko (2000) sprechen von kooperierenden Lehrkräften als „discourse communities“ (S. 8), in denen vorhandenes Wissen geteilt und so aufeinander bezogen wird, dass neues Wissen entsteht. In diesem Sinne wird Kooperation große Bedeutung für Innovationsprozesse zugesprochen. Holtappels, Lossen, Spillebeen und Tillmann (2011) bezeichnen Lehrerkooperation als „Schlüsselvariable für die Entwicklung von Kapazitäten einer lernenden Organisation“ (S. 27). Lernen durch Austausch wird bei einem anspruchsvollen Beruf wie dem Lehrberuf für notwendig erachtet (vgl. ebd.; siehe auch Wilson \& Berne, 1999). Von multiprofessioneller Kooperation kann erwartet werden, dass die Kompetenzerweiterung und die Entstehung neuen Wissens durch die Verschiedenartigkeit des Wissens und der Zugänge zum professionellen Handlungsfeld in noch stärkerem Maße stattfinden und dass von ihr Impulse für Innovationsprozesse durch Gewinn neuer Perspektiven ausgehen. Der Frage, unter welchen Bedingungen sich diese Effekte einstellen, wird im nächsten Abschnitt nachgegangen.

Besonderheiten multiprofessioneller schulischer Kooperation. Dass multiprofessionelle Kooperation an Schulen sowohl nötig als auch wünschenswert ist, scheint in der Schulforschung allgemeiner Konsens zu sein. Über die Zusammenarbeit zwischen Lehrkräften mit anderen Berufsgruppen sind aber noch keine vergleichbar umfassenden theoretischen Analysen vorgenommen worden wie über Lehrerkooperation (siehe hierzu Boller, 2012). An dieser Stelle werden deshalb zunächst Besonderheiten multiprofessioneller gegenüber monoprofessioneller Kooperation an Schulen dargestellt. Diese werden dann am Beispiel der Kooperation mit der Jugendhilfe und der Sonderpädagogik illustriert und mit professionstheoretischen Überlegungen verknüpft, bevor abschließend Diskurslinien skizziert werden.

Meist werden in einschlägigen Publikationen die mit Konfliktpotential behafteten Herausforderungen berufsübergreifender Kooperation thematisiert, in allgemeiner Form z. B. von Schütze (1999), wenn er schreibt, dass „die Arbeitsteilung der Professionen mit anderen Professionen und nicht-professionellen Berufen stets ein prekäres Aushandlungsergebnis“ (S. 196) ist. Diese Annahme scheinen viele Autor/innen, die sich theoretisch (und/oder empirisch) mit der Verhältnisbestimmung von Schule bzw. Regelschullehrkräften und spezifischen anderen Berufsgruppen auseinandersetzen, zu teilen (Arndt et al., 2014; Arnoldt, 2008a; Breuer, 2011; Hedderich \& Hegner, 2009; Hillenbrand, 2012; Holtappels, 2008; Speck, 1988, Speck et al., 2011a). Aushandlungsbedürftig sind etwa das professionelle Verständnis von Erziehung und Bildung sowie die Rollenzuschreibungen und Zuständigkeiten in der Zusammenarbeit. Konfliktpotential bieten dabei wahrgenommene oder reale Statusunterschiede, die sich auch im Gehalt und der beruflichen Absicherung ausdrücken, Differenzen im professionellen Selbstverständnis und das Teilen von Entscheidungsmacht und Gratifikation. Hinzu kommen können gegenseitige Verstehensprobleme aufgrund beruflicher Fachsprachen, und, insbesondere im schulischen Kontext, auf Individualebene Unsicherheiten und Berührungsängste und auf Organisationsebene die fehlende strukturelle Einbindung von nicht festangestelltem 
Personal und allgemeiner Mangel an Kooperationsstrukturen, wie festen Zeiten und Räumlichkeiten sowie Berücksichtigung der Mehrarbeit für kooperatives Arbeiten (Arndt et al., 2014; Arnoldt, 2008a; Hedderich \& Hegner, 2009; Lütje-Klose, 2011; Lütje-Klose \& Willenbring, 1999; Schneider, 2004; Speck, 1988). Für Lehrkräfte lässt sich aufgrund der theoretischen Analysen zu Lehrerkooperation erwarten, dass Unsicherheit in der multiprofessionellen Zusammenarbeit insbesondere in der Deprivatisierung ihrer Berufsausübung liegt. Für Vertreter/innen anderer Professionen kann eine Unsicherheit darin liegen, sich als Nicht-Lehrkräfte in die vorhandene schulische Struktur zu integrieren und sich dabei möglicherweise „häufig einem geschlossen wirkenden Kollegium gegenüber“ (Dieckmann, Höhmann \& Tillmann, 2008, S. 183) zu sehen. Statusunterschiede kommen dabei nicht nur in der direkten Zusammenarbeit in impliziter oder expliziter Form zum Tragen, sondern drücken sich auch in der formalen Einbindung bzw. der Nicht-Einbindung in schulische Entscheidungsgremien aus (vgl. Arnoldt, 2008a). Häufig wird im Zusammenhang mit multiprofessioneller Kooperation an Schulen deshalb Gleichberechtigung der Kooperationspartner/innen gefordert (z. B. ebd.), wobei die entscheidungsmächtigere Position der Schule bzw. den Lehrkräften zugeschrieben wird (z. B. Breuer, 2015; Holtappels, 2008).

Im Fall der Zusammenarbeit von Jugendhilfe und Schule wird der Jugendhilfe oft eine „Kompensationsund Komplementfunktion“ (Holtappels, 2008, S. 499) bzw. eine „,nachgeschaltet-dienende[n]“ (ebd., S. 500) Funktion zugeschrieben. Demnach werden Dienste der Jugendhilfe bzw. der Schulsozialarbeit vor allem im außerunterrichtlichen Bereich und in der Einzelfallhilfe zur Intervention im Falle „schwieriger Problemlagen und auffälliger Schüler" (ebd., S. 501) in Anspruch genommen. Damit verbunden ist eine Entlastung der Lehrkräfte gerade im sozial-erzieherischen Aufgabenbereich, aber keine konzeptuelle Zusammenarbeit zwischen Schule und Jugendhilfe (ebd.). Während Schulen und Lehrkräften die Aufgabe zukommt, anhand von Leistungskriterien zu differenzieren, sind das Jugendamt und Schulsozialarbeiter/innen für Integration und Teilhabe zuständig (Hillenbrand, 2012). Vor diesem Hintergrund liegt die Annahme nahe, dass es zwischen beiden Institutionen und ihren Vertreter/innen eher zu Abgrenzung als zu Annäherung kommt. Der verstärkte Integrationsanspruch an Schule, der insbesondere an Ganztagsschulen gestellt wird, macht die eindeutige Festlegung von Zuständigkeitsgrenzen jedoch zunehmend schwerer (Rahm et al., 2015). Die Aufweichung vormals fester Grenzen zwischen Professionen und Disziplinen, die als Kennzeichen der heutigen Arbeits- und Wissenschaftswelt unter dem Schlagwort Entgren₹ungsdebatte diskutiert wird, verlegt die Aushandlung von Zuständigkeiten auf Akteursebene, wie Rahm et al. (2015, S. 146) feststellen: „Ehemals unhinterfragt geltende Grenzen lösen sich auf und können nur über Absprache unter den Beteiligten in der jeweiligen Situation abgesteckt werden“. Die Autor/innen diskutieren in diesem Zusammenhang die Notwendigkeit von „Grenzpolitik“ (ebd.) und einem „kontinuierlichen disziplinübergreifenden Fachdiskurs“ (ebd.).

Auch in der Fachdiskussion um das Verhältnis von Schulpädagogik und Sonderpädagogik spielt die Frage nach den Zuständigkeiten eine große Rolle. Kron (2012) hält das Verhältnis beider Disziplinen vor allem in Ländern wie Deutschland und Frankreich, die „starke Zweige sonderpädagogischer Einrichtungen“ (S. 156) ausgebildet haben, für problematisch. Speck (1998) spricht sogar von einem ,verdeckten oder offenen Berufsgruppenkampf um das behinderte Kind“ (S. 419). Durch die Ausweitung inklusiver Beschulung haben diese Fragen neue Dynamik erhalten: Die Zuständigkeiten werden nun in vermehrter Zahl nicht mehr über die Zuordnung zu der einen oder anderen Schulform entschieden, sondern müssen in der kollegialen Zusammenarbeit geklärt werden (siehe hierzu auch Lütje-Klose \& Willenbring, 1999), was durch das weitgehende Fehlen übergeordneter verbindlicher Standards zum inklusiven Unterricht erschwert wird (Gebhard et al., 2014). Insofern gilt verstärkt auch für Regelschullehrkräfte und sonderpädagogische Lehrkräfte, dass sich „geltende Grenzen“ (Rahm et al., 2015, S. 146) auflösen und Bildung- und Erziehungsverständnisse möglicherweise neu verhandelt werden müssen. Während Regelschullehrkräfte sich bei der Gestaltung des Unterrichts und bei der Leistungsbeurteilung eher an der Sachnorm und der Sozialnorm orientieren, richten sonderpädagogische Lehrkräfte ihre Arbeit mit den Schüler/innen stärker an der individuellen Norm aus (Arndt et al., 2014; Lütje-Klose \& Willenbring, 1999). Zusätzlich erschwerend kann sich eine Ungleichheit in der strukturellen und sozialen Einbindung ins Kollegium und die schulischen Gremien auswirken, die insbesondere in dem - häufigen - Fall auftritt, dass sonderpädagogische Lehrkräfte nur stundenweise an der Regelschule arbeiten (Lütje-Klose, 2011).

Zur Begegnung der Herausforderungen multiprofessioneller Kooperation werden auf individueller Ebene häufig die Offenheit für die Reflektion der eigenen Berufsrolle und die Perspektivübernahme in der Zusammenarbeit für wichtig erachtet (Arndt et al., 2014; Speck et al., 2011a). Auf institutioneller Ebene 
werden meist die Herstellung von Gleichberechtigung der Kooperationspartner/innen etwa durch Einbindung in schulische Organisations- und Entscheidungsstrukturen gefordert sowie eine Schaffung von Strukturen, die Kooperation ermöglichen und absichern (Arnoldt, 2008a; Hedderich \& Hegner, 2009; Speck et al., 2011a, 2011b). Die Klärung der Zuständigkeitsfrage halten einige Autor/innen auf institutioneller Basis für nötig, so z. B. Melzer, Hillenbrand, Sprenger und Hennemann (2015, S. 76): „Klare Aufgabenbeschreibungen $[\ldots]$ bieten den Vorteil einer transparenten Basis für eine gelingende Kooperation verschiedener Professionen [...]. Nicht zuletzt beugen klare Aufgabenbeschreibungen und Kompetenzprofile der Entstehung unproduktiver Konkurrenzsituationen vor.“ Rahm et al. (2015) haben dagegen eine eher flexible Handhabung der Zuständigkeiten im Blick, sie sprechen von „reflexive[n] Kooperationsmodelle[n], in denen je nach Situation und Expertise jeweils neu entschieden werden muss" (ebd., S. 146f.). Die Bedeutung von professioneller Expertise betonen Arndt et al. (2014), wenn sie davor warnen, dass „Interesse und persönliche Kompetenzen" keine hinreichende Grundlage für die Zuteilung von Aufgabenbereichen seien und stattdessen ,in Richtung funktionaler Ausdifferenzierung der Rollen zu denken ist" (ebd., S. 35).

\subsubsection{Konzeptualisierungen und Modelle schulischer Kooperation}

Zur Systematisierung kooperativer Tätigkeiten und ihrer Einbettung in größere schulische Zusammenhänge sind verschiedene Konzeptualisierungen und Modelle entwickelt worden. Den Beitrag von Kooperation zur Wirksamkeit von Schule und für Innovationsprozesse beschreiben das Schulqualitätsmodell von Ditton (2000) bzw. das Schulentwicklungsmodell von Rolff (2010). Die systematische Beschreibung von Tätigkeiten, die sich als kooperativ bezeichnen lassen, wird durch spezielle Unterscheidungen verschiedener Kooperationsformen ermöglicht. Die meisten dieser Konzeptualisierungen oder Modelle nehmen spezifische Zusammenhänge der jeweiligen Kooperationsformen mit verschiedenen Rahmenbedingungen oder mit verschiedenen Wirkungsaspekten an. Die wichtigsten sowohl der übergeordneten Schulmodelle, die Kooperation als eine Variable beinhalten, als auch der Konzeptualisierungen und Modelle von Kooperation werden im Folgenden vorgestellt. Die Darstellung beschränkt sich dabei auf die theoretischen Annahmen der Modelle; soweit empirische Befunde zu den Modellen vorliegen, finden sie sich im nachfolgenden Teilkapitel.

Im Qualitätsmodell von Schule nach Ditton (2000) nehmen kooperationsbezogene Merkmale eine zentrale Stellung ein. Das Modell differenziert nach Voraussetzungen (etwa die personellen und finanziellen Bedingungen und die Lehrpläne), primären Merkmalen auf Schul- und Unterrichtsebene sowie Wirkungen (etwa Schülerleistungen und Einstellungen). „Kooperation und Koordination“ ist in diesem Modell einer von vier Qualitätsfaktoren, die auf Schulebene wirksam sind und umfasst einen koordinierten Schul- und Unterrichtsbetrieb, Kooperation mit Partner/innen außerhalb der Schule, die Einführung neuer Lehrer/innen und den Austausch von Erfahrungen und Wissen. Auch die anderen drei Qualitätsfaktoren auf Schulebene stehen in inhaltlichem Zusammenhang mit kooperativer Praxis. So stehen hinter dem Faktor „Schulkultur“ etwa gemeinsame Ziele, Leitung und geregelte Zuständigkeiten, der Faktor „Schulmanagement“ beinhaltet eine gemeinsame Vision und geregelte Aufgabenverteilungen und der Faktor „Personalpolitik und Personalentwicklung" umfasst unter anderem die Kooperation zwischen Akteur/innen innerhalb der Schule. Auch im Schulentwicklungsmodell von Rolff (2010) spielt Kooperation eine zentrale Rolle. Schulentwicklung hat als Ziel und Bezugspunkt den Lernfortschritt der Schüler/innen und wird als Trias von Unterrichts-, Personal- und Organisationsentwicklung konzeptualisiert. Sowohl Organisationsentwicklung als auch Personalentwicklung beinhaltet eine Stärkung der kooperativen Strukturen, in der Organisationsentwicklung explizit benannt als „Kooperation“ und ,TEAMENTWICKLUNG“ (Hervorhebung im Original) und in der Personalentwicklung z. B. als Supervision/Coaching, Kommunikationstraining, Hospitationen, Jahresgespräche.

Konkreter als in den beschriebenen Schulmodellen wird die Kooperation auf Schulebene oder auf Ebene der handelnden Individuen in speziellen Konzeptualisierungen und Modellen von schulischer Kooperation erfasst. Mit Ausnahme des Modells von Marvin (1990) beziehen sich die vorliegenden Modelle auf Lehrerkooperation und kategorisieren vor allem Tätigkeiten der unterrichtsbezogenen Zusammenarbeit. Lässt man die konkreten Tätigkeiten, die den Kategorien beispielhaft zugeordnet werden, unberücksichtigt, eignen sich die Kategorienmerkmale aber durchaus auch zur Kategorisierung multiprofessioneller Kooperation. Vergleichbar ausgearbeitete Modelle, die explizit multiprofessionelle Kooperation beschreiben, liegen für die Schulforschung noch nicht vor; auch begrifflich hat sich multiprofessionelle Kooperation in der Schulforschung noch nicht etabliert (Boller, 2012). 
Allgemeine Konzeptualisierungen zur Beschreibung von Kooperation liefern Kuper und Kapelle (2012), Ahlgrimm, Krey und Huber (2012) und Esslinger (2002). Kuper und Kapelle (2012) benennen folgende Strukturdimensionen für die Beschreibung von Kooperation auf Organisationsebene: Arbeitsteilung, Koordination, Hierarchien, Delegation und Formalisierung. Ahlgrimm et al. (2012) unterscheiden zwischen verschiedenen Kooperationsverständnissen: Wird Kooperation als Vertragsverbältnis verstanden, ist sie durch äußere Erfordernisse bedingt; wird Kooperation als Einstellung verstanden, ist ihr Zustandekommen von individuellen Dispositionen abhängig. Kooperation als Arbeitsteilung wiederum wird bestimmt durch die Notwendigkeit von Arbeitsorganisation und-abstimmung und Kooperation als Strategie durch die Beschaffenheit von Zielen. Esslinger (2002) unterscheidet zwischen struktureller Kooperation, bei der durch Austausch die individuelle Arbeit optimiert und die „Funktionsfähigkeit des vorhandenen Systems“ (S. 73) gestützt wird, und integrativer Kooperation, die prozessorientiert auf die „Entwicklung von gemeinsamen Ideen, Materialien, Profilen und Konzepten" (S. 74) abzielt.

Neben diesen eher an ihren Funktionen orientierten Konzeptualisierungen wurden verschiedene Stufenmodelle von Kooperation entwickelt. Die Modelle von Rolff (1980) sowie von Steinert et al. (2006) kategorisieren Kooperation nach dem Stand ihrer Ausprägung auf Schulebene und gehen von einer hierarchisch gedachten Entwicklung von weniger zu mehr Kooperation aus. Die Modelle von Little (1990, zitiert nach Fussangel, 2008), Marvin (1990) sowie Gräsel et al. (2006) und Fussangel (2008) beschreiben Kooperation nach dem Grad ihrer Intensität bzw. Elaboriertheit auf Individualebene.

Rolff (1980) nimmt mehrere Unterscheidungen von Kooperationsformen vor. Zum einen grenzt er horizontale Kooperation als Zusammenarbeit von Statusgleichen von vertikaler Kooperation als Zusammenarbeit von Personen, die in einem Hierarchieverhältnis zueinander stehen, ab und zum anderen gefügeartige von teamartiger Kooperation. Die schulischen Rahmenbedingungen (siehe Lortie, 1975; Weick, 1976) lassen seinen Annahmen nach eher gefügeartige Kooperation, d. h. ein zwar koordiniertes, aber letztlich relativ unverbundenes Arbeiten, und weniger teamartige Kooperation, d. h. eine unmittelbare Zusammenarbeit, zu. Diese unmittelbare Zusammenarbeit kann eher technisch (mit dem Ziel der Zeitersparnis durch Arbeitsteilung) oder pädagogisch (durch inhaltliche Auseinandersetzung mit Unterrichtsstoff) ausgestaltet sein. Bezüglich der teamartigen Kooperation nimmt Rolff (1980) an, dass je nach Umfang und Schwierigkeitsgrad der kooperativen Tätigkeiten bestimmte Entwicklungsschritte durchlaufen werden. Diese Annahme erlaubt es, Schulen nach dem Stand ihrer Entwicklung teamartiger Kooperation in eine Rangfolge zu bringen. Ein hierarchisches Entwicklungsmodell für die Ausprägung von Kooperation bzw. des „systematischen, wechselseitig adaptiven und integrierten Lehrerhandelns" (Steinert et al., 2006, S. 195) an Schulen nehmen auch Steinert et al. (2006) an und bringen es in Verbindung mit dem Stand der Schulentwicklung. Das Fehlen von Kooperation wird als Fragmentierung bezeichnet, die erste Niveaustufe von Kooperation als Differenzierung, gefolgt von Koordination, Interaktion und, als vierter und höchster Niveaustufe, Integration. Je höher die Stufe, desto detaillierter beispielsweise das gemeinsame Zielkonzept und desto höher die systematische Abstimmung und Zusammenarbeit bei der Unterrichtsplanung und Unterrichtsdurchführung.

Auf Ebene der handelnden Individuen unterscheidet Little (1990, zitiert nach Fussangel, 2008) zwischen vier Stufen von Lehrerkooperation, die im Grad der Autonomie, d. h. der wechselseitigen Abhängigkeit der Beteiligten, variieren. Bei hoher Autonomie spricht sie von Storytelling and Scanning for Ideas; auf dieser Stufe findet ein unverbindlicher Austausch statt. Auf der Stufe mit dem nächstgeringeren Autonomiegrad, Aid and Assistance, wird auf freiwilliger Basis Unterstützung geleistet. Erst auf der Stufe Sharing findet bewusst arbeitsbezogener Austausch statt und nur auf der Stufe mit dem geringsten Autonomiegrad, Joint Work, werden arbeitsbezogene Entscheidungen gemeinsam getroffen (Little, 1990, zitiert nach Fussangel, 2008). Ein ähnliches Modell entwirft Marvin (1990) zur schulischen Kooperation von Regelschullehrkräften und Sprachheilpädagog/innen. Sie bezeichnet Kooperation, bei der Reziprozität und Vertrauen niedrig ausgeprägt sind, als Co-activity, ein relativ unverbundenes Nebeneinander der jeweiligen Tätigkeiten. Auf den Stufen mit den nächsthöheren Graden an Reziprozität und Vertrauen findet Informationsaustausch statt (Cooperation) und Austausch auch von Meinungen und Material (Coordination). Auf der Stufe hoch ausgeprägten Vertrauens und Reziprozität (Collaboration) verständigen sich die Beteiligten über die jeweiligen Verantwortlichkeiten und Rollen bei der gemeinsamen Arbeitsaufgabe. Von Stufe zu Stufe steigt dabei auch der Zeitumfang, der in Kooperation investiert wird. Trotz der Benennung distinkter Kategorien geht Marvin davon aus, dass kooperative Beziehungen sich auf einem Kontinuum zwischen den Polen niedrige bzw. hohe Reziprozität/niedriges bzw. hohes Vertrauen befinden.

Aufbauend auf die Kooperationsdefinition von Spieß (2004) und das Stufenmodell von Little (1990, zitiert nach Fussangel, 2008) differenzieren Gräsel et al. (2006; siehe auch Fussangel, 2008) zwischen drei 
Kooperationsformen: Austausch (fachlich oder schülerberogen), Arbeitsteilung/Arbeitsteilige Organisation und Kokonstruktion/Reflektion. Die Formen unterscheiden sich im Ausprägungsgrad der gemeinsamen Ziele bzw. Aufgaben, des Vertrauens und der Autonomie, d. h. darin, inwieweit sie die Bedingungen von Kooperation nach Spieß (2004) realisieren (Fussangel, 2008; Gräsel et al., 2006). Insofern lassen sie sich als abgestuft intensive Formen von Kooperation bezeichnen, die jeweils andere Funktionen erfüllen (Fussangel, 2008). Mit Austausch wird das gegenseitige Versorgen mit unterrichtsbezogenen oder schülerbezogenen Informationen bezeichnet, wofür weder eine bedeutsame Einschränkung der eigenen Autonomie noch eine gemeinsame Bindung an ein konkretes Ziel nötig ist (ebd.). Diese Form von Kooperation ist relativ unverbindlich und birgt aufgrund ihrer geringen Intensität wenig Potential für Konflikte; sie stellt eine „low-cost“-Form von Kooperation dar (Gräsel et al., 2006, S. 210). Arbeitsteilung liegt vor, wenn eine Aufgabe anteilig von zwei oder mehr Personen bearbeitet wird. Notwendig dafür ist eine Abstimmung über die Aufteilung und das angestrebte Arbeitsergebnis sowie Vertrauen in die angemessene Bearbeitung durch die anderen. Eine direkte Zusammenarbeit bei der Aufgabendurchführung ist jedoch nicht nötig (Gräsel et al., 2006), so dass hierbei eine gewisse Autonomie gewahrt bleibt. Zuletzt beschreiben die Autor/innen Kokonstruktion als elaborierte Form der Kooperation: „Kokonstruktion liegt dann vor, wenn die Partner sich intensiv hinsichtlich einer Aufgabe austauschen und dabei ihr individuelles Wissen so aufeinander beziehen (kokonstruieren), dass sie dabei Wissen erwerben oder gemeine Aufgaben- und Problemlösungen entwickeln“ (ebd., S. 210f.). Diese Form von Kooperation schränkt die Autonomie der Beteiligten stärker ein als die beiden anderen, sie erfordert die Abstimmung nicht nur über das Arbeitsergebnis, sondern auch über den Arbeitsprozess. Die gemeinsame Reflektion erfordert die Bereitschaft, die eigene Arbeit und die der anderen offen zu diskutieren, was im Vergleich mit den anderen Kooperationsformen einen höheren zeitlichen Aufwand bedeutet, ein höheres Konfliktpotential birgt („high-cost“, ebd., S. 211) und höheres Vertrauen voraussetzt. Kooperation im Sinne von Kokonstruktion ist die Form von Kooperation, von der positive Effekte für die Professionalisierung von Lehrkräften und für schulische Innovationen erwartet werden (Fussangel, 2008; Gräsel et al., 2006). In dem Kooperationsmodell von Fussangel (2008, siehe Abbildung 1) werden die drei beschriebenen Kooperationsformen als Kooperationspraxis gefasst und in Bezug gesetzt zu den Rahmenbedingungen für Kooperation und zu Nutzenaspekten von Kooperation, die aus qualitativen Interviews mit Lehrkräften heraus formuliert wurden: Die Rahmenbedingungen umfassen die Kooperationsbereitschaft der Kolleg/innen, gegenseitige Sympatbie, Zeitstrukturen, ein gemeinsames Ziel und den Zwang zur Kooperation. Als Nutzenaspekte beinhaltet das Modell emotionale Entlastung, fachlicher Nutzen zur eigenen Weiterbildung, verbesserter Scbülerfokus und Arbeitsentlastung. Fussangel (2008) nimmt an, dass die Rahmenbedingungen für Kooperation einen direkten Einfluss auf die Kooperationspraxis und den Nutzen von Kooperation haben, unabhängig davon hat auch die Kooperationspraxis Einfluss auf den Nutzen.

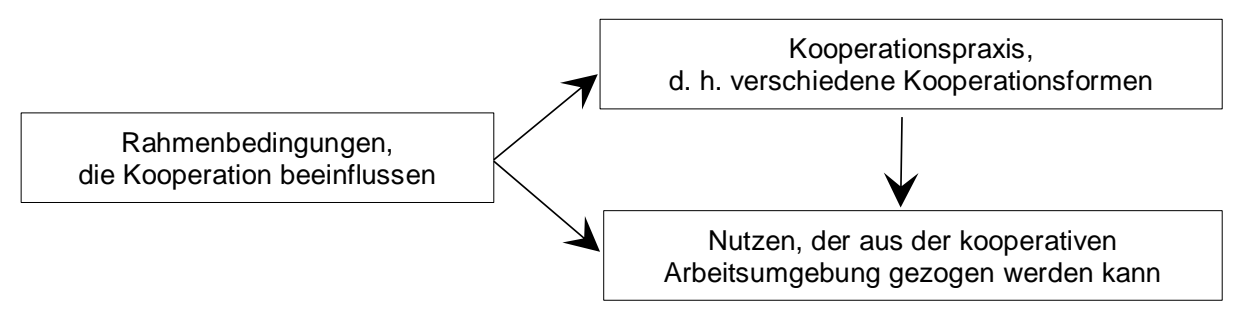

Abb. 1: Kooperationsmodell von Fussangel (2008, S. 190)

\subsection{Forschungsstand}

Im Folgenden wird der Forschungsstand zum Thema Lehrerkooperation und zu multiprofessioneller Kooperation im Schulkontext aufgearbeitet. Obwohl beide Formen von Kooperation insbesondere durch die Ganztagsschulforschung wieder stärker in den Fokus bildungswissenschaftlicher Forschung gerückt sind, bildet Lehrerkooperation nach wie vor ein empirisch wie theoretisch weitaus besser untersuchtes Forschungsfeld als multiprofessionelle Kooperation an Schulen. Um aus den Erkenntnissen über Lehrerkooperation Ansatzpunkte für die Untersuchung multiprofessioneller Kooperation an Schulen zu entwickeln, wird der Lehrerkooperation an dieser Stelle ein eigenes Unterkapitel gewidmet. Ein besonderer Fokus liegt auf 
den empirischen Ergebnissen zu dem im vorherigen Teilkapitel beschriebenen Kooperationsmodell von Gräsel et al. (2006) und Fussangel (2008).

Zu multiprofessioneller Kooperation an Schulen liegen Untersuchungen zur Zusammenarbeit von Regelschullehrkräften mit sonderpädagogischen Lehrkräften sowie mit Schulsozialarbeiter/innen, Erzieher/innen und weiterem pädagogisch tätigen Personal an Ganztagsschulen vor. Da zur Kooperation mit Schulbegleiter/innen bislang kaum geforscht wurde (die wenigen Ergebnisse hierzu sind im vorherigen Kapitel berichtet), sollen aus den vorliegenden Studien zu multiprofessioneller Kooperation in der Schule Ansatzpunkte für die Zusammenarbeit von Lehrkräften und Schulbegleiter/innen entwickelt werden. Zur Kooperation von Lehrkräften untereinander und mit anderen Berufsgruppen kann auf zahlreiche Studien im deutschen Raum zurückgegriffen werden; aufgrund der landeseigenen Spezifika von Schulsystemen konzentriert sich die Ausarbeitung des Forschungsstands im Wesentlichen auf diese Untersuchungen und wird nur bei Bedarf durch internationale Forschungsergebnisse ergänzt.

\subsubsection{Lehrerkooperation}

Häufige Untersuchungsgegenstände in der Kooperationsforschung bilden der Inhalt und das Ausmaß von Lehrerkooperation, Zusammenhänge mit Merkmalen der Akteure und mit schulischen Rahmenbedingungen sowie Nutzen und Wirkungen von Kooperation. Der folgende Überblick über empirische Erkenntnisse zu Lehrerkooperation ist entsprechend dieser inhaltlichen Aspekte gegliedert. Da nicht nur verschiedene Konzeptualisierungen von Kooperation es erschweren, die Forschungsergebnisse systematisch aufeinander zu beziehen (vgl. z. B. Fussangel, 2008), sondern auch Unterschiede im Untersuchungsdesign (quantitativ bzw. qualitativ), werden an dieser Stelle - entsprechend der Anlage der eigenen Studie - vor allem quantitativ angelegte Studien berücksichtigt und qualitative Studien explizit als solche benannt.

Inhalte und Ausmaß. Dem Kerngeschäft des Lehrerberufs gemäß wird Kooperation in vielen Studien vor allem auf das Unterrichten bezogen. Darunter fallen eher einfache Tätigkeiten der Zusammenarbeit wie der Austausch von Material, aber auch die Abstimmung von Inhalten und komplexere Formen der Kooperation wie gemeinsame Unterrichtsplanung und -durchführung. Quantitative wie qualitative Befunde der Schulforschung stützen insgesamt die Annahme, dass insbesondere komplexe Formen unterrichtsbezogener Kooperation an Schulen nicht in ausgeprägtem Maße stattfinden (Bauer, 2008; Böhm-Kasper, 2004; Dizinger et al., 2011a, 2011b; Drossel \& Willems, 2014; Ehlers, Jude, Klieme \& Helmke, 2008; Esslinger, 2002; Fussangel \& Gräsel, 2012; Richter \& Pant, 2016; Rolff, 1980; Steinert et al., 2006; Steinert, Harting \& Klieme, 2008; Ulich, 1996).

Im Rahmen der DESI-Studie wurde festgestellt, dass die in den einzelnen Fachkollegien am häufigsten praktizierten Kooperationsformen der Austausch von Material und die Abstimmung von Zielsetzungen ist, während didaktische Kooperation oder gegenseitige Hospitationen seltener vorkommen (Steinert et al., 2008). Insgesamt gibt die Hälfte der befragten Deutschlehrkräfte an, in irgendeiner Form mit den Fachkolleg/innen zusammenzuarbeiten (Ehlers et al., 2008). In der Studie von Esslinger (2002) berichtet jeweils etwa ein Viertel der Lehrkräfte von Erfahrungen in der gemeinsamen Materialanfertigung und Unterrichtsplanung sowie der Abstimmung von Unterrichtsinhalten, wohingegen nur ein Zehntel Erfahrungen im gemeinsamen Unterrichten haben. Zu dem Ergebnis, dass weniger aufwändige Kooperationsformen überwiegen, kommt auch Fussangel (2008) bei der Befragung von 168 Lehrkräften: Demnach findet Austausch häufiger statt als arbeitsteilige Organisation und am wenigsten häufig Kokonstruktion (für eine Beschreibung dieser Kooperationsformen siehe Kapitel 3.1.5). Auf ihr Kooperationsmodell bauen auch die Studien von Dizinger (2015) und Richter und Pant (2016) auf, die jeweils einen Stichprobenumfang von $n>1000$ aufweisen, und zu dem gleichen Ergebnis führen. Richter und Pant (2016) setzen die Ergebnisse außerdem in Bezug zu internationalen kooperationsbezogenen Ergebnissen der TALIS-Studie der OECD ${ }^{19}$. Auch in der TALIS-Studie wird eine Differenzierung von Lehrerkooperation nach Komplexität vorgenommen, indem zwischen Exchange and Co-ordination und Professional Collaboration unterschieden wird, und es wird länderübergreifend ein höheres Ausmaß an weniger komplexen Kooperationstätigkeiten gegenüber komplexen Kooperationstätigkeiten festgestellt (OECD, 2009, 2014). Auf Grundlage des Vergleichs von Einzelitems, d. h. der Häufigkeit spezifischer Kooperationstätigkeiten, berichten Richter und Pant (2016), dass Lehrkräfte in Deutschland beispielsweise häufiger Material austauschen als im OECD-Durchschnitt und deutlich sel-

19 Deutschland hat sich an der TALIS-Studie nicht beteiligt. 
tener die Lernentwicklung ihrer Schüler/innen besprechen oder gemeinsame Standards zur Leistungsbewertung entwickeln. Im Schnitt arbeiten deutsche Lehrkräfte 4.9 Wochenstunden mit Kolleg/innen zusammen bei einer durchschnittlichen Wochenarbeitszeit von 43 Stunden. Damit liegen sie über dem OECDSchnitt von 2.9 Stunden, allerdings liegt auch die durchschnittliche Wochenarbeitszeit (die in beiden Fällen Voll- und Teilzeitkräfte einschließt) mit 38 Stunden niedriger (OECD, 2014; siehe auch Burns \& DarlingHammond, 2014).

Auch für ganze Schulen gilt, dass elaborierte Formen von Kooperation eher selten vorkommen. Steinert et al. (2006) befragten 158 Schulen in Hessen und Zürich und ordneten sie anhand der Ergebnisse auf eine der Niveaustufen von Lebrerkooperation (siehe Kapitel 3.1.5) ein. Demnach befinden sich 53\% der Schulen auf der ersten Niveaustufe Differenzierung, 22\% auf der zweiten Niveaustufe Koordination, 13\% auf der dritten Niveaustufe Interaktion und 2\% auf der vierten Niveaustufe Integration. Keinerlei Kooperationsstrukturen (Fragmentierung) herrschen bei 10\% der Schulen vor.

Zusammenhänge mit Merkmalen auf Individual- und Schulebene. Obwohl die verschiedenen Studien schulformübergreifend keine hohe Kooperationsintensität berichten, gilt dies für die einzelnen Schulformen in unterschiedlichem Maße. Generell scheint Kooperation auf Schulebene mit der Ausprägung von Kollegialität bzw. Kohäsion im Lehrerkollegium zusammenzuhängen (Ahlgrimm et al., 2012; Lossen, Rollett \& Willems, 2013), sowohl Umfang als auch Intensität von Kooperation nehmen aber mit der Höhe der formalen Abschlüsse der Schulart offensichtlich ab (Fussangel \& Gräsel, 2012). Für Gymnasien werden in Studien regelmäßig die geringsten Ausprägungen von Kooperation gefunden (Bauer, 2008; Ehlers et al., 2008; Fussangel \& Gräsel, 2012; Steinert et al., 2006) und an Grundschulen finden sich höhere Ausprägungen als an weiterführenden Schulen (Dieckmann et al., 2008). Gesamtschulen als Schulen mit vergleichsweise hoher Kooperationsausprägung fallen aus diesem Muster heraus (Bauer, 2008; Ehlers et al., 2008; Steinert et al., 2008). Dagegen unterscheidet sich die Kooperation an Ganztagsschulen entgegen der Erwartungen, die an diese Schulform gestellt werden, insgesamt kaum von Halbtagsschulen (Fussangel \& Gräsel, 2012), Richter und Pant (2016) stellen aber für vollgebundene Ganztagsschulen teilweise höhere Ausprägungen kooperativer Tätigkeiten fest. In der gleichen Studie berichten die Autoren häufigere Kooperation sowohl im Sinne von Austauschtätigkeiten als auch im Sinne komplexerer unterrichtsbezogener Kooperation an Schulen, die inklusiven Unterricht anbieten.

Die Schulformunterschiede lassen darauf schließen, dass schulische Merkmale eine Rolle für die Ausprägung von Kooperation spielen. Richter und Pant (2016) finden zudem Zusammenhänge zwischen Kooperationsaktivitäten und der Etablierung von Zeitstrukturen zur Kooperation sowie der Unterstützung von Kooperation durch die Schulleitung. Ähnliche Befunde berichten auch Drossel und Willems (2014). Nach den Ergebnissen der TALIS-Studie hängt die Ausprägung von Kooperation aber in wesentlich höherem Maße von individuellen Merkmalen ab (OECD, 2014). Lehrkräfte, die zufrieden mit ihrem Beruf sind und sich darin als kompetent erleben, kooperieren sowohl in einfacher wie aufwändiger Form häufiger (ebd.). Diese Zusammenhänge zeigen Richter und Pant (2016) auch für die deutsche Stichprobe. Zu vergleichbaren Ergebnissen kommen Dizinger et al. (2011a) bzw. Pröbstel und Soltau (2012), die einen positiven Zusammenhang zwischen der berufsbezogenen Selbstwirksamkeitserwartung bzw. dem beruflichen Selbstwert von Lehrkräften und der Ausprägung der Kooperationsformen nach Gräsel et al. (2006) bzw. Fussangel (2008) berichten. Den stärksten Einfluss auf das Kooperationsverhalten finden Pröbstel und Soltau (2012) aber für eine soziale Orientierung der Beteiligten und eine gemeinsame Zielbindung. Ebenso kommt Fussangel (2008) im Rahmen der Überprüfung ihres Kooperationsmodells (siehe Kapitel 3.1.5) zu dem Ergebnis, dass das gemeinsame Ziel der stärkste Prädiktor für Austausch, Arbeitsteilung und Kokonstruktion ist. Signifikante Zusammenhänge zu diesen drei Kooperationsformen zeigen sich auch für die gegenseitige Sympathie, nicht aber für die Kooperationsbereitschaft der Kolleg/innen und die Freiwilligkeit der Kooperation. ${ }^{20}$ Weitere untersuchte Merkmale im Zusammenhang mit Kooperation sind aus theoretischen Überlegungen zum Lehrerindividualismus abgeleitet (siehe Kapitel 3.1.2): Entgegen den Erwartungen zeigt sich bei Pröbstel und Soltau (2012) keine besonders hohe Ausprägung des Autonomiebedürfnisses unter den befragten Lehrkräften (siehe hierzu auch Soltau, Berthe \& Mienert, 2012, für ähnliche Befunde in einer anderen Stichprobe) und weder das Autonomiebedürfnis noch die Angst vor negativer Bewertung stehen in Zusammenhang mit

20 Der Faktor Zeitstrukturen als Rahmenbedingung für Kooperation (der sich eher der Schul- als der Individualebene zuordnen lässt) sowie die Kooperationsform Schülerbezogener Austausch werden von der Autorin im Rahmen der Modellüberprüfung eliminiert, weshalb hierüber keine Zusammenhangsergebnisse vorliegen (Fussangel, 2008). 
Kooperation (Pröbstel \& Soltau, 2012). In einigen Studien wurden Lehrkräfte direkt nach ihrer Einstellung zu Kooperation befragt. Esslinger (2002) berichtet, dass kooperationsoffene Lehrkräfte tendenziell berufszufriedener sind und sich stärker mit ihrer Schule identifizieren (siehe hierzu auch Holtappels et al., 2011). Unterschiede ergeben sich auch in Abhängigkeit von dem eigenen Professionsverständnis: Kooperationsoffene Lehrkräfte verstehen sich stärker als Erzieher/innen als kooperationsablehnende Lehrkräfte, die sich stärker als Wissensvermittler/innen sehen (Esslinger, 2002). Dass Einstellungen allerdings nur bedingt mit Handeln zusammenhängen, zeigt sich in der Studie daran, dass der Anteil an Lehrkräften, der Kooperation auf verschiedenen unterrichts- und schülerbezogenen Gebieten für praxisrelevant halten, jeweils bedeutend höher ist als der Anteil derjenigen, die auf diesen Gebieten Kooperationserfahrungen haben. Eine hohe Zustimmungsrate von über $90 \%$ der Befragten hinsichtlich der Bedeutsamkeit von kollegialer Kooperation dokumentieren auch Richter und Pant (2016). Alters- und Geschlechtseffekte finden sich in einigen Studien (z. B. bei Esslinger, 2002, für die höhere Ausprägung kooperationsgünstiger Einstellungen bei jüngeren und bei weiblichen Lehrkräften; siehe auch Ulich, 1996), in anderen nicht oder nicht in einheitlicher Weise (Fussangel, 2008; Pröbstel \& Soltau, 2012).

Ziele und Nutzen. Von Kooperation unter Lehrkräften werden in der Regel vor allem positive Wirkungen erwartet. Für die Beteiligten werden Entlastung durch soziale Unterstützung und Arbeitsentlastung sowie Professionalisierung durch Wissenszuwachs erwartet und auf Schulebene eine erhöhte Effektivität.

Vor dem Hintergrund, dass Lehrkräfte ihren Beruf einerseits in relativer Isolation von Kolleg/innen ausüben und andererseits statistisch gesehen eine Risikogruppe für Burnout bilden, wurde unter anderem untersucht, inwieweit Kooperation ein Schutzschild gegen Belastungen im Lehrerberuf bilden kann. In der Untersuchung von Schaarschmidt (2005) zeigt sich soziale Unterstützung im Schulalltag, insbesondere durch die Unterstützung durch die Schulleitung und ein positives soziales Klima im Kollegium, als wichtigster entlastender Faktor. In eine ähnliche Richtung weisen auch die Befunde von Böhm-Kasper (2004). Neben dem positiven Einfluss auf das Wohlbefinden, der von sozialer Unterstützung allgemein angenommen werden kann, kann kollegiale Zusammenarbeit auch über die Entwicklung realistischer Ansprüche an die eigene Arbeit entlastend wirken (vgl. Fussangel, 2008): Empirisch sind unrealistische Ansprüche und Erwartungen an die eigene Arbeit, die nach Schaarschmidt (2006) gerade in sozialen Berufen eine Herausforderung darstellen, mit einem erhöhten Burnoutrisiko assoziiert (Schmitz \& Leidl, 1999). Die Höhe komplexer Anforderungen ist aber kein genereller Gradmesser für Belastung; auch Monotonie kann nachweislich belastend wirken (Ulich, 1996). Fussangel (2008) findet in der empirischen Überprüfung ihres Kooperationsmodells einen Einfluss der Kooperationsformen Austausch, Arbeitsteilung und Kokonstruktion auf das Erleben emotionaler Entlastung. Diese Zusammenhänge werden auch in Interviews mit Lehrkräften hergestellt (Dizinger, 2015), lassen sich aber bei anderer Operationalisierung der Entlastung in Nachfolgestudien nicht quantitativ nachweisen (Böhm-Kasper, Gräsel \& Weishaupt, 2009; Dizinger, 2015; Dizinger et al., 2011a, 2011b). Neben emotionaler Entlastung misst Fussangel (2008) auch Arbeitsentlastung durch Kooperation; hierbei zeigen sich nur für die Kooperationsform Austausch bedeutsame Zusammenhänge.

Zusammenfassend kann festgehalten werden, dass die Befunde zu Kooperation und Belastung nicht einheitlich ausfallen, aber in der Tendenz erwartungsgemäß in dem Sinne, dass Kooperation zu Entlastungserleben führt (für einen Überblick siehe Dizinger, 2015). Belastende Momente von Kooperation, wie etwa erhöhter Zeitaufwand und die erhöhte Gefahr von Konflikten insbesondere bei high-cost-Formen von Kooperation (siehe Kapitel 3.1.5), scheinen quantitativ nicht von Bedeutung zu sein. Aufschlussreich für das Verständnis des Zusammenhangs von Kooperation und Wohlbefinden sind die Ergebnisse der qualitativen Interviewstudie von Ahlgrimm (2012): Es zeigt sich, dass bei wenig Zusammenarbeit die beruflichen Beziehungen zwischen Lehrkräften vielfach von Unsicherheit und Angst geprägt sind, unter anderen bedingt durch das Fehlen von sichtbaren Vergleichsmaßstäben zur Beurteilung des eigenen Handelns. Engere Zusammenarbeit fördert dagegen Sicherheit, Akzeptanz und Kollegialität und trägt zum professionellen Weiterlernen bei (siehe hierzu auch Wilson \& Berne, 1999). In eine ähnliche Richtung weisen die Befunde von Esslinger (2002), nach denen kooperationsablehnende Einstellungen vor allem bei Lehrkräften auftreten, die über wenig Erfahrung mit Kooperation verfügen.

Aufgrund der in Kapitel 3.1.3 vorgestellten Überlegungen wird von Kooperation auch ein positiver Effekt auf die Professionalisierung von Lehrkräften erwartet. Diesem haben sich insbesondere qualitative Arbeiten gewidmet (z. B. Bondorf, 2013; für einen Überblick siehe Wilson \& Berne, 1999). Im quantitativen Bereich lässt sich wiederum auf Fussangel (2008) verweisen, die für die Kooperationsformen Austausch, Arbeitsteilung und Kokonstruktion eine positive Wirkung auf den fachlichen Wissenszuwachs nachweist. 
Vergleichbare Ergebnisse verschiedener nationaler wie internationaler Studien berichten zusammenfassend auch Holtappels et al. (2011) und in der TALIS-Studie (OECD, 2014) werden Zusammenhänge gefunden zwischen der Häufigkeit verschiedener schüler- und unterrichtsbezogener Kooperationstätigkeiten mit kooperativen Aktivitäten, die explizit der Weiterentwicklung der eigenen professionellen Praxis dienen, wie etwa die gegenseitige Hospitation im Unterricht zur Feedbackgebung und gegenseitiges Coaching.

Auf Schulebene wird Kooperation mit einer höheren Schuleffektivität, verstanden als Lernzuwachs der Schüler/innen, assoziiert. Scheerens, Glas und Thomas (2003) nehmen auf Grundlage internationaler Studien professionelle Kooperation als Merkmal effektiver Schulen an. Obwohl die Annahme plausibel scheint, dass Lehrerkooperation, vermittelt etwa über die bessere Abstimmung von Inhalten und konsistentem Lehrerhandeln, zu besseren Lernleistungen der Schüler/innen führt, konnte empirisch bislang kein direkter Zusammenhang zwischen beiden Variablen nachgewiesen werden (Steinert et al., 2006, 2008).

\subsubsection{Multiprofessionelle Kooperation}

Unabhängig von Schulbegleiter/innen lassen sich grob drei Akteursgruppen skizzieren, die aktuell neben Regelschullehrkräften in Regelschulen arbeiten: Sonderpädagogische Lehrkräfte, Schulsozialarbeiter/innen (in der Regel Sozialpädagog/innen) und weiteres pädagogisch tätiges Personal an Ganztagsschulen. ${ }^{21}$ Je nach Tätigkeitsbereich ergeben sich unterschiedliche Anlässe zur Kooperation mit den Regellehrkräften, die im Folgenden dargestellt werden. Zunächst werden Befunde zur Kooperation mit sonderpädagogischen Lehrkräften berichtet und anschließend Befunde zur Kooperation mit weiterem pädagogisch tätigen Personal. Hierunter werden auch Sozialpädagog/innen gefasst, die in der Schulsozialarbeit tätig sind. Neben quantitativen Studien werden auch Ergebnisse qualitativer Studien vorgestellt.

Kooperation mit sonderpädagogischen Lehrkräften. Sonderpädagogische Lehrkräfte arbeiten entweder festangestellt an Regelschulen (etwa in Integrationsklassen) oder stundenweise im Rahmen von Schulkooperationen oder als Teil des Mobilen Sonderpädagogischen Dienstes (Lütje-Klose, 2011). Sie sind im Speziellen zuständig für die Schüler/innen mit sonderpädagogischem Förderbedarf; Kooperation mit den Regelschullehrkräften findet vor allem bezogen auf die Zusammenarbeit im Unterricht und die Förderung einzelner Schüler/innen statt (ebd.).

Im Rahmen integrativer Schulmodelle und Schulkooperationen wurden schon Mitte der 1970er Jahre Erfahrungen mit der Kooperation von Regelschullehrkräften und sonderpädagogischen Lehrkräften gesammelt (Lütje-Klose, 2011). Befragungen von kooperierenden sonderpädagogischen Lehrkräften aus den 1980er Jahren ergaben, dass die Kooperation verschiedene Belastungsfaktoren mit sich bringt (Brähler, 1991). Dabei werden die Berufsrolle, unterschiedliche pädagogische Vorstellungen und mangelnde Wertschätzung thematisiert (ebd.). Die befragten sonderpädagogischen Lehrkräfte sehen sich an Regelschulen teilweise mit der Erwartung konfrontiert, sämtliche Probleme mit schwierigen Schüler/innen zu lösen, und mit der Notwendigkeit, ihr Aufgabengebiet und ihre Rolle immer wieder auszuhandeln (ebd.). Ein ähnliches Bild zeichnen auch aktuelle Studien, die die Rollenunklarheit insbesondere der sonderpädagogischen Lehrkraft im Gemeinsamen Unterricht als Erschwernis für gelingende Kooperation ergeben (Gebhard et al., 2014; Lütje-Klose \& Willenbring, 1999; Pool \& Moser Opitz, 2014; Wessel, 2005).

Gebhard et al. (2014) erfassten mit Fragebögen Daten von insgesamt 245 Regel- und sonderpädagogischen Lehrkräften, die in inklusiven Settings arbeiten, und finden, dass die Kooperation zwar von beiden Seiten insgesamt positiv beurteilt wird (über $80 \%$ sind zufrieden oder eher zufrieden), die Zufriedenheitswerte der sonderpädagogischen Lehrkräfte aber in fast allen Aspekten geringer ausfallen. Im Vergleich empfinden die sonderpädagogischen Lehrkräfte weniger Arbeitsentlastung durch die Kooperation, ihnen sind die Aufgaben und Ziele der Zusammenarbeit weniger klar und sie beurteilen den Teamzusammenhalt negativer. Darüber hinaus sehen sie die Kooperation weniger als Bereicherung für die Schüler/innen und fühlen sich weniger imstande, ihnen im Gemeinsamen Unterricht gerecht werden zu können. Die Autor/innen vermuten als Ursache für diese relative Unzufriedenheit mit dem Gemeinsamen Unterricht, dass sonderpädagogische Lehrkräfte höhere Ansprüche an den Gemeinsamen Unterricht stellen, da er bereits in ihrer Ausbildung ein wichtiger Bestandteil ist. Die relative Unzufriedenheit mit der Kooperation wird unter anderem auf die zeitliche Mehrbelastung zurückgeführt, da die sonderpädagogischen Lehrkräfte mit mehr Regelschullehrkräften in direkter Zusammenarbeit stehen als umgekehrt. Zusammengenommen schwanken

21 Verwaltungs- und Reinigungskräfte bleiben hierbei unberücksichtigt. 
die Angaben zum zeitlichen Umfang von Teamgesprächen zwischen wenigen Minuten bis mehreren Stunden pro Woche. Im Schnitt werden hierfür wöchentlich etwa 90 Minuten aufgewendet, die vor allem „Teamsitzungen“ und „Gespräche zwischen Tür und Angel“" (Gebhard et al., 2014, S. 22) ausmachen. Inhaltlich geht es dabei vor allem Themen, die ,eher dringlich“ sind (S. 28), wie organisatorische Absprachen und die Befindlichkeit der Schüler/innen. Übergeordnete Themen wie Didaktik und die Zusammenarbeit an sich werden seltener besprochen. ${ }^{22}$ Unterschiedsanalysen ergeben, dass diejenigen Regel- und sonderpädagogischen Lehrkräfte, die sich mit der Kooperation zufrieden zeigen, häufiger auf freiwilliger Basis zusammenarbeiten als aus Zwang, mehr Zeit für gemeinsame Gespräche aufwenden und sich häufiger über die genannten übergeordneten Themen austauschen. Die Autor/innen schließen aus den Ergebnissen, dass die Kooperationsqualität bei den mit der Kooperation unzufriedenen Lehrkräften niedriger ist. Für kritisch halten sie, dass den Angaben der Gesamtstichprobe nach zwar „weitgehend“ (S. 29) Klarheit über die Ziele der Kooperation herrscht, aber „kaum“ Kriterien zur Zielerreichung vorliegen (ebd.). Vor diesem Hintergrund verweisen die Autor/innen auf die Notwendigkeit übergeordneter verbindlicher Standards zur inklusiven Beschulung, deren Fehlen es den jeweils Beteiligten auferlege, diese zu entwickeln. Lütje-Klose und Willenbring (1999) berichten in diesem Zusammenhang von Fallbeispielen, in denen sich die schwierige Vereinbarkeit des schulpädagogischen Anspruchs, mit der ganzen Klasse bestimmte Lernziele zu erreichen, und des sonderpädagogischen Anspruchs der Förderung leistungsschwacher Schüler/innen in schwerlich zu lösenden Kooperationsproblemen niederschlägt.

Ein letzter Aspekt zur Kooperation von Regelschullehrkräften und sonderpädagogischen Lehrkräften sei an dieser Stelle noch erwähnt: Offenbar kann insbesondere arbeitsteilige Kooperation im Unterricht bei Schüler/innen zu der Wahrnehmung von sonderpädagogischen Lehrkräften als „Differenzmarker“ (Arndt et al., 2014, S. 29) führen. In der Fallanalyse von Arndt et al. (2014) kommt die Differenzierung zwischen leistungsschwachen und leistungsstarkern Mitschüler/innen anhand der Zuordnung zur Sonderpädagogin zum Ausdruck, als ein Schüler (ohne sonderpädagogischen Förderbedarf) sagt: „Deniz sollte kein FrauFriedrichs-Kind sein [...], Deniz find ich ist schon sehr schlau“" (S. 30).

Kooperation mit weiterem pädagogisch tätigen Personal. Sozialpädagogische Fachkräfte sind im Rahmen der Schulsozialarbeit schon wesentlich länger an Schulen tätig als das weitere pädagogisch tätige Personal (kurz: weiteres pädagogisches Personal) bzw. die Honorarkräfte, die im Zuge des Ausbaus von Ganztagsschulen beschäftigt werden.

Schulsozialarbeiter/innen übernehmen an Schulen verschiedenste Aufgaben, von der Hausaufgabenbetreuung bis zur Drogenprävention. Untersuchungen zeigen, dass sie in der Regel zu einem großen Teil mit einzelnen, problematischen oder auffälligen Schüler/innen beschäftigt sind - und in diesem Rahmen mit den jeweiligen Lehrkräften, Eltern und ggf. weiteren beteiligten Institutionen (z. B. dem Jugendamt) zusammenarbeiten - und mit der Gestaltung von Angeboten im außerunterrichtlichen Bereich (Holtappels, 2008). Eine konzeptuelle Zusammenarbeit findet insgesamt eher weniger statt (ebd.). In Ganztagsschulen überschneidet sich der Aufgabenbereich der Schulsozialarbeit mit dem des weiteren pädagogischen Personals bzw. werden sozialpädagogische Fachkräfte als Teil dieses Personals im Ganztag tätig: Als weiteres pädagogisch tätiges Personal werden in der Ganztagsforschung alle die Mitarbeiter/innen bezeichnet, die pädagogisch im Ganztag tätig sind, aber nicht als Lehrkräfte an der Schule angestellt sind (Höhmann, Bergmann \& Gebauer, 2008). Sie werden entweder von der Schule oder von außerschulischen Kooperationspartner/innen der Schule (z. B. Jugendhilfe, Sportvereine, Musikschulen) eingesetzt. Da der Großteil aktueller Forschung multiprofessionelle Kooperation im Rahmen von Ganztagsschule untersucht, wird im Folgenden nicht unterschieden zwischen Schulsozialarbeiter/innen und anderen Personen des weiteren pädagogisch tätigen Personals.

Zu dieser Personengruppe wurden in der StEG-Studie in der ersten Erhebungsphase (beginnend 2005) umfassende Daten erhoben und ausgewertet, von denen die wichtigsten an dieser Stelle zusammengefasst werden. Vom Qualifikationshintergrund her ist das weitere pädagogisch tätige Personal sehr heterogen (Arnoldt, 2008b). Insgesamt besteht es etwa zur Hälfte aus pädagogisch ausgebildeten Kräften (Höhmann et al., 2008). Daneben arbeiten z. B. Sportübungsleiter/innen und Studierende an Ganztagsschulen (Arnoldt, 2008b). Etwa 10\% verfügen über keine abgeschlossene Ausbildung, etwa 50\% über eine Berufsausbildung und $40 \%$ haben ein Studium abgeschlossen (Höhmann et al., 2008). Je nach Schulform arbeiten zwischen 10 und 20\% ehrenamtlich (ebd.). Schulformunterschiede zeigen sich im formalen Qualifikationsgrad, der an

22 Ähnlich berichtet auch Brähler (1991), dass aufwändige Formen der unterrichtsbezogenen Zusammenarbeit wie Team-Teaching eher selten stattfinden. 
Sekundarschulen höher liegt als an Grundschulen, und im Anteil der hauptberuflich Angestellten, der mit $71 \%$ an Grundschulen höher liegt gegenüber 44\% an Sekundarschulen (ebd.). An Grundschulen bilden Erzieher/innen mit 41\% die größte Berufsgruppe und an Sekundarschulen sozialpädagogische Fachkräfte mit 13\% (ebd.). Im Schnitt kommen auf 100 Schüler/innen an Grundschulen 6 Lehrkräfte und 5.5 Personen des weiteren pädagogischen Personals, die durchschnittlich jeweils etwa 32 Stunden wöchentlich dort arbeiten, und an weiterführenden Schulen 8.2 Lehrkräfte und 1.8 Personen des weiteren pädagogisch tätigen Personals mit durchschnittlich jeweils etwa 10 Wochenarbeitsstunden (ebd.). Über die Hälfte dieser Personengruppe ist für weniger als 50\% der regulären Arbeitszeit eingestellt (Arnoldt, 2008b), viele über befristete Verträge (Steiner \& Tillmann, 2011). Vom Umfang der Wochenarbeitszeit her dominieren insgesamt die pädagogischen Fachkräfte bzw. als außerschulische Kooperationspartnerin die Jugendhilfe (Arnoldt, 2008b).

Mit der Bedeutung einer pädagogischen Fachqualifikation für die Arbeit im Ganztag setzen sich die Analysen von Steiner und Tillmann (2011) und Steiner (2013) auseinander. Anhaltspunkte hierzu liefern Befunde, nach denen pädagogischen Fachkräfte in intensiverem Austausch mit den Lehrkräften stehen als pädagogische Laien; außerdem sehen sie einen höheren Veränderungsbedarf im Ganztag und empfinden sich stärker als Teil der Schulgemeinschaft (Steiner \& Tillmann, 2011). Daneben lassen sich jedoch keine ausgeprägten Unterschiede zwischen beiden Gruppen finden, zumal viele Laien pädagogische Kompetenzen durch Berufserfahrungen im pädagogischen Bereich oder durch Fortbildungen erworben haben (Steiner, 2013). Steiner (2013) bezeichnet diese Gruppe als „professionalisierte Laien“ (S. 68) und resümiert, dass „ein professioneller Habitus nicht zwangsläufig an formale Abschlüsse gebunden ist“ (S. 86).

Was Inhalt und Ausmaß von Kooperation zwischen Lehrkräften und dem weiterem pädagogisch tätigen Personal betrifft, deuten die Befunde der StEG-Studie darauf hin, dass insgesamt wenig fachlicher Austausch stattfindet; auch gibt nur die Hälfte der außerschulischen Kooperationspartner/innen an, dass ihre Angebote mit dem Unterricht verknüpft seien (Steiner \& Tillmann, 2011). Auch die Ergebnisse der MUKUS-Studie (Lehmann-Wermser et al., 2010) verweisen auf mangelnde Einbindung der außerschulischen Kooperationspartner/innen, was sich z. B. darin niederschlägt, dass tagesaktuelle Informationen wie Raumwechsel nicht an sie weitergeleitet werden.

Kooperationsbezogene Unterschiede finden sich in der StEG-Studie sowohl auf Individualebene als auch hinsichtlich der Schulform: Länger tätiges bzw. pädagogisch ausgebildetes Personal arbeitet tendenziell mehr bzw. intensiver mit Lehrkräften zusammen (Steiner \& Tillmann, 2011). An Grundschulen ist die Zusammenarbeit intensiver als an weiterführenden Schulen und das weitere pädagogisch tätige Personal wird stärker eingebunden (Tillmann \& Rollett, 2011). Die Schulformunterschiede treten auch in der zweiten Erhebungsphase von StEG in Erscheinung: Grundschulen verfügen deutlich häufiger über festgelegte Kooperationszeiten für Lehrkräfte und das weitere pädagogische Personal als weiterführende Schulen, unter denen die Gymnasien am seltensten über festgelegte Kooperationszeiten verfügen (Klieme, Rauschenbach, Holtappels \& Stecher, 2015).

Die Kooperation mit dem weiteren pädagogischen Personal wurde auch im Zusammenhang mit der strukturellen Einbindung sowie hinsichtlich ihrer Bewertung durch die Beteiligten untersucht. Dabei stellte sich heraus, dass die Einbindung in schulische Planungs- und Entscheidungsprozesse kooperationsfördernd ist, aber keinen Einfluss auf die Bewertung der Kooperation durch das weitere pädagogische Personal nimmt (Tillmann \& Rollett, 2011). Im Schnitt wird die Zusammenarbeit von den Lehrkräften leicht positiver beurteilt als vom weiteren pädagogisch tätigen Personal; beide Werte liegen aber auf einem guten Niveau, wobei sich keine Unterschiede zwischen den Schulformen zeigen (Dieckmann et al., 2008). An Grundschulen und vollgebundenen Ganztagsschulen fühlt sich das weitere pädagogische Personal jedoch stärker eingebunden (ebd.), auch die Länge der Tätigkeit an einer Schule erhöht die Einschätzung der Eingebundenheit (Steiner \& Tillmann, 2011). Insgesamt sieht 86\% des weiteren pädagogisch tätigen Personals Verbesserungsbedarf im Bereich Kooperation (ebd.). Auch die außerschulischen Kooperationspartner/innen als solche zeigen sich insgesamt zufrieden mit der Zusammenarbeit mit der Schule, wobei sich schulformübergreifend die Wahrnehmung als gleichberechtigt im Kooperationsverhältnis als stärkster Prädiktor für die Zufriedenheit zeigt (Arnoldt, 2008a). Die Wahrnehmung von Gleichberechtigung fällt höher aus, wenn die Kooperationspartner/innen selbst der Kooperation einen hohen Stellenwert beimessen und wenn ihre Angebote mit dem Unterricht verknüpft sind. Dagegen haben die Teilnahme an Gremien und der Kooperationsvertrag nur bedingt Einfluss auf die Wahrnehmung von Gleichberechtigung und die Zufriedenheit mit der Kooperation (ebd.). 
Neben der StEG-Studie sind im Rahmen des Forschungsprojekts Formen der Lehrerkooperation und Beanspruchungserleben an Ganztagsschulen Untersuchung zur multiprofessionellen Kooperation an Sekundarganztagsschulen durchgeführt worden (Dizinger, 2015; Dizinger \& Böhm-Kasper, 2012; Dizinger et al., 2011a, 2011b). In Interviews gaben die Lehrkräfte dabei an, dass vor allem die Verknüpfung von Unterricht mit außerunterrichtlichen Angeboten und schulische, soziale oder familiäre Probleme von Schüler/innen Anlässe zur Kooperation mit fest angestellten sozialpädagogischen Kräften seien (Dizinger \& Böhm-Kasper, 2012). Die Zusammenarbeit bestehe dabei in der Regel aus kurzen Gesprächen und der Abgabe von Aufgaben oder Zuständigkeiten durch die Lehrkräfte an die sozialpädagogischen Fachkräfte. Elaborierte Formen von Zusammenarbeit werden kaum berichtet. Die Lehrkräfte geben an, durch die Kooperation mit den sozialpädagogischen Kräften Arbeitserleichterung und, vor allem bei Schülerproblemen, emotionale Entlastung zu erfahren. Außerdem bringe die Kooperation Vorteile für die Schüler/innen. Aufbauend auf die Interviewergebnisse und auf das Kooperationsmodell von Fussangel (2008) wurden Skalen zur quantitativen Befragung von Lehrkräften entwickelt, die schülerbezogene von unterrichtsbezogener Zusammenarbeit abgrenzen. Da in den Interviews fast ausschließlich über Austauschtätigkeiten berichtet wird, wird nicht zwischen verschiedenen Intensitätsstufen in der Kooperation unterschieden. Die Ergebnisse der Fragebogenstudie zeigten, dass mehr schülerbezogene als unterrichtsbezogene Kooperation mit dem weiteren pädagogisch tätigen Personal stattfindet (Dizinger \& Böhm-Kasper, 2012). Die Ausprägung schülerbezogenen Austausches korreliert hoch mit der ebenfalls neu entwickelten Skala Entlastung durch Kooperation (Dizinger, 2015) und schwach (aber signifikant) mit fachlichem Nutzen und emotionaler Entlastung, die angelehnt an Fussangel (2008) operationalisiert wurden. Unterrichtsbezogene Kooperation korreliert nur, und ebenfalls schwach, mit fachlichem Nutzen. Kaum messbare Zusammenhänge bestehen zwischen unterrichts- und schülerbezogenem Austausch mit den erhobenen Merkmalen von Belastungserleben, emotionaler Erschöpfung, Dehumanisierung, reduzierter Leistungsfähigkeit und somatischer Beschwerden (Dizinger \& BöhmKasper, 2012). Lediglich schülerbezogener Austausch steht in schwachem negativen Zusammenhang zu reduzierter Leistungsfähigkeit (ebd.). Zusammenhangsanalysen zwischen den beiden multiprofessionellen Kooperationsformen und den drei Formen von Lehrerkooperation Austausch, Arbeitsteilung und Kokonstruktion nach Gräsel et al. (2006) bzw. Fussangel (2008) zeigen durchgängig schwache bis mittlere positive Korrelationen auf, insbesondere zwischen unterrichtsbezogener Kooperation mit dem weiteren pädagogischen Personal und den beiden elaborierteren Lehrerkooperationsformen Arbeitsteilung und Kokonstruktion (Dizinger \& Böhm-Kasper, 2012).

Ergänzend zu den vorgestellten stärker quantitativ ausgerichteten Studien kommen an dieser Stelle noch zentrale Ergebnisse qualitativer Studien (Böttcher et al., 2011; Breuer, 2015; Olk, Speck \& Stimpel, 2011) zur Sprache, die Aufschluss über die Ausgestaltung multiprofessioneller Kooperationsbeziehungen insbesondere auf Personenebene bieten. Olk et al. (2011) arbeiten in ihren qualitativen Schulfallstudien heraus, dass das Verhältnis von Lehrkräften und dem weiteren pädagogisch tätigen Personal mit dem Verhältnis von Schule und Schulsozialarbeit vergleichbar ist. Auf Schulebene könne insgesamt von einer ,,umfassend reflektierten und geplanten Kooperation [...] bisher nur in Ausnahmefällen gesprochen werden“ (S. 76). Der gemeinsamen Konzeptentwicklung werde wenig Aufmerksamkeit geschenkt, wodurch mögliche Differenzen in den Erwartungen an die Kooperation im Vorfeld nicht thematisiert werden. Das Potential multiprofessioneller Kooperation für schulische Innovationsprozesse werde auf diese Weise nicht ausgeschöpft, stattdessen komme es zu einem „Nebeneinanderher verschiedener Berufsgruppen am Ort der Schule“ (ebd.), die sich zwar untereinander abstimmen, aber nicht in einen fachlichen Dialog treten. Auf Personenebene zeigen sich stärker differenzierte Kooperationsbeziehungen. In der Zusammenarbeit von Lehrkräften und dem weiteren pädagogisch tätigen Personal finden die Autoren sowohl stark hierarchiegeprägte Konstellationen, in denen die Lehrkräfte in der Zusammenarbeit zwar eine Arbeitsentlastung für sich sehen, aber keine Ansatzpunkte für fachliche Kooperation, die eine Veränderung ihres bisherigen Arbeitsverständnisses und ihrer Rolle bedeuten könnte. Daneben werden aber auch kooperative Beziehungen gefunden, in denen „offene Aushandlungsprozesse hinsichtlich der Arbeitsteilung und der Übernahme von Aufgaben“ (S. 75) und eine Auseinandersetzung mit den unterschiedlichen „Sichtweisen und methodischen Herangehensweisen“ (ebd.) innerschulischer Partner/innen stattfinden. Dabei werden sowohl „Abgrenzungen der professionellen Domänen vorgenommen“ (ebd.) als auch „,neue Formen der Arbeitsteilung und Zusammenarbeit entwickelt" (S. 75f.).

Breuer (2011, 2015) untersucht die Kooperation von Teams aus Lehrkräften und Erzieher/innen an Ganztagsgrundschulen anhand der qualitativen Auswertung von Teamsitzungen. Die Aufgabenbereiche der 
Erzieher/innen können dabei sowohl außerunterrichtliche Angebote und Hilfestellungen wie auch die Mitarbeit im Unterricht beinhalten, je nach schulspezifischer oder teamspezifischer Ausgestaltung. Die Auswertungen zeigen, dass Kooperation als „Differenzierung von Zuständigkeit“ (Breuer, 2011, S. 85) gefasst werden kann, wofür Breuer (2015) drei Muster identifiziert: Kooperation entlang von Hauptzuständigkeit und Zuarbeit, Kooperation entlang der Entdifferenzierung von Zuständigkeiten und Kooperation entlang fachbezogener Zuständigkeiten. Ihre Befunde verdeutlichen, dass nicht jede multiprofessionelle Zusammenarbeit zu Perspektivenübernahme und Kompetenzerweiterung führt (siehe auch Reh \& Breuer, 2012). $\mathrm{Zu}$ ähnlichen Ergebnissen kommen auch Böttcher et al. (2011). Die Zusammenarbeit zwischen den von ihnen interviewten Ganztagslehrkräften und dem weiteren pädagogischen Personal lässt sich im Wesentlichen als komplementär beschreiben. Der Unterricht ist Aufgabenbereich der Lehrkräfte, der außerunterrichtliche Bereich ist dem weiteren pädagogisch tätigen Personal zugeordnet. Zwischen beidem gibt es wenig Verknüpfung, die Befragten zeigen sich mit der Zusammenarbeit aber zufrieden.

\subsection{Zusammenfassung}

Im ersten Teil dieses Kapitels wurden zunächst Definitionsansätze von Kooperation und anschließend zentrale Aspekte der Kooperation im Schulbereich dargestellt. In diesem Zusammenhang wurde auf die Besonderheiten und die Veränderungen schulischer Rahmenbedingungen für Kooperation eingegangen und die Verbindung zwischen Kooperation, Professionalisierung und Innovation diskutiert. Dabei wurde deutlich, dass der seit etwa zwei Jahrzehnten anhaltende Reformprozess im Schulsystem zu deutlich mehr Bedarf an professioneller und multiprofessioneller Kooperation an Schulen geführt hat. Hiervon werden Impulse zur professionellen Weiterentwicklung der eigenen Berufsrolle und der eigenen beruflichen Praxis erwartet, wobei insbesondere multiprofessionelle Kooperation mit Herausforderungen verbunden ist, die vermuten lassen, dass die erhofften und möglichen positiven Effekte sich nicht ohne weiteres einstellen. Anhand der vorgestellten Modelle zu schulischer Kooperation wird darüber hinaus deutlich, dass Formen von Kooperation unterscheidbar sind, die jeweils eigenen Zwecken dienen und unterschiedliche Wirkungen entfalten.

Die berichteten Forschungsergebnisse zu Kooperation an Schulen bieten einen Überblick über die tatsächliche Ausprägung von Kooperation an Schulen und ihre Zusammenhänge mit verschiedenen Merkmalen und Nutzenaspekten. Insgesamt scheint die Annahme bestätigt, dass vor allem unterrichtsbezogen wenig Kooperation an Schulen stattfindet, was für die Kooperation mit weiterem pädagogischen Personal in noch stärkerem Maße gilt als für Lehrerkooperation (Böttcher et al., 2011; Fussangel, 2008; Steiner \& Tillmann, 2011; Steinert et al., 2006). Die empirischen Befunde liefern aber zahlreiche Ansatzpunkte für eine differenzierte Betrachtung der Situation. Zum einen kann die Annahme, dass verschiedene Kooperationsformen empirisch unterschieden werden können, als bestätigt gelten (Drossel \& Willems, 2014; Fussangel, 2008; Richter \& Pant, 2016; Steinert et al., 2008), zum anderen können verschiedenartige Zusammenhänge zwischen der Ausprägung und der Ausgestaltung von Kooperation und individuellen und schulischen Merkmalen sowie Nutzenaspekten ausgemacht werden (Drossel \& Willems, 2014; Fussangel, 2008; Dizinger \& Böhm-Kasper, 2012). Insbesondere die Erforschung auch problematischer Aspekte von Kooperation, die gerade im Zusammenhang mit multiprofessioneller Kooperation häufig im Zentrum steht (z. B. Brähler, 1991; Breuer, 2015; Gebhard et al., 2014; Lütje-Klose \& Willenbring, 2011; Olk et al., 2011), liefert wichtige Erkenntnisse über die tatsächliche Kooperationspraxis an Schulen mit dem Fokus auf den handelnden Subjekten. 


\section{Zusammenfassung und Forschungsdesiderate}

In Kapitel 2 wurden zunächst rechtliche Rahmenbedingungen und ihre Umsetzung in Stadt und Landkreis Göttingen beschrieben. Die anschließend dargestellten zentralen Punkte aktueller Diskussionen zeigen deutlich, dass Schulbegleitung als Maßnahme der Eingliederungshilfe nicht auf befriedigende Weise in ein Gesamtsystem inklusiver Beschulung eingebettet ist (z. B. AGJ, 2014; Böing, 2013; Dworschak, 2010; Knuf, 2013). Die empirischen Befunde, die bislang vorliegen, stützen die Annahmen, dass Schulbegleitung nicht ausschließlich von pädagogischen Fachkräften geleistet wird und dass eine Trennung schulischer Aufgaben von den Tätigkeiten, die Schulbegleiter/innen ausführen, schwer möglich ist (Beck et al., 2010; Dworschak, 2012b, 2012c; Henn et al., 2014; Lindmeier et al., 2014; Wohlgemuth, 2009). Trotz zahlreicher Hinweise aus qualitativen Studien auf Schwierigkeiten bei der Klärung von Zuständigkeiten und Rollenverständnissen (Böing, 2013; Heinrich \& Lübeck, 2013; Lindmeier et al., 2014; Lübeck \& Heinrich, 2016) zeigen sich die Beteiligten in quantitativ angelegten Studien insgesamt zufrieden mit der Maßnahme (Dworschak, 2012b, 2012c; Wohlgemuth, 2009; Zauner \& Zwosta, 2014). Die Nichtpassung von Qualifikation und der Verantwortung für unterrichtliche Instruktion, die Rollenunklarheit und, in einigen Studien, die positiven Zufriedenheitswerte finden sich auch in den Ergebnissen internationaler Studien zu nichtprofessionellem Assistenzpersonal im inklusiven Unterricht (Butt \& Lowe, 2012; Hanover Research, 2010; Köpfer, 2013a, 2013b; Webster et al., 2010, 2011). Evaluationsstudien zum Einsatz von Assistenzpersonal verdeutlichen dabei die Bedeutung der jeweils für den gemeinsamen Unterricht zuständigen Lehrkräfte für die professionelle Anleitung und Kooperation (Blatchford et al., 2009a, 2009b).

Die insgesamt dünne Studienlage speziell zu Schulbegleitung lässt viele Fragen offen. Zum einen existieren bislang keine verallgemeinerbaren Zahlen zur näheren Beschreibung der Maßnahme und der Schulbegleiterschaft; vorhandene Studien beziehen sich jeweils auf spezifische Regionen. Für den Göttinger Raum liegen bislang keine Übersichten etwa über die Häufigkeit der Maßnahme, die Verteilung auf Schulformen und häufige Aufgabenfelder von Schulbegleitung vor. Auch zu der Population der lokalen Schulbegleiter/innen, wie ihrem Qualifikationshintergrund und ihrer Motivation, liegen keine Daten vor. Insbesondere fehlt es an Studien, die theoretisch fundierte Analysen zu Schulbegleitung in der Praxis vornehmen. Die Diskussionen, die um Schulbegleitung geführt werden, bieten reichlich Ansatzpunkte für mögliche Fragestellungen. Vor dem Hintergrund, dass häufig die unzureichende konzeptionelle Einbindung von Schulbegleitung in das System Schule bemängelt wird, erscheint die nähere Untersuchung der Kooperation mit Schulbegleitungen auf Schul- und Unterrichtsebene bedeutsam. Die Ergebnisse internationaler Forschung zu Assistenzpersonal liefern Hinweise, dass von der Untersuchung der schulischen Einbindung und der Kooperation mit den Lehrkräften, mit denen sie gleichzeitig im Unterricht tätig sind, lohnenswerte Erkenntnisse für die schulische Praxis ausgehen können (Webster, Blatchford \& Russell, 2013a). Bisherige Studien zu Schulbegleitung nähern sich dem Thema Kooperation wenn, dann vorrangig auf deskriptivem Niveau 
oder in Form qualitativer Dokumentation. Da für die Kooperation mit Schulbegleiter/innen oder Assistenzkräften bislang keine Modelle ausgearbeitet wurden, an die eine empirische Untersuchung anknüpfen könnte, widmete sich das folgende Kapitel der theoretisch wie empirisch bereits besser untersuchten Kooperation von Lehrkräften untereinander und mit Vertreter/innen anderer Professionen an der Schule.

Die in Kapitel 3 vorgestellten theoretischen Analysen und empirischen Befunde zu Kooperation an Schulen zeigen, dass insbesondere Ganztagsschulen und die Ausweitung inklusiver Beschulung zu einer erhöhten Zahl an Kooperationsanlässen auch mit anderen Professionen geführt haben. Die theoretisch herausgearbeiteten Besonderheiten der schulischen Rahmenbedingungen und der Herausforderungen multiprofessioneller Kooperation finden sich in der empirischen Befundlage wieder. Kooperation ist oftmals kein schulstrukturell gesicherter Teil der Arbeit von Lehrkräften und findet häufig nur in Form von Austauschund Abspracheprozessen statt (Fussangel, 2008; Richter \& Pant, 2016; Steiner \& Tillmann, 2011; Steinert et al., 2006). Kooperation in dem Sinne, dass die Beteiligten in einen fachlichen Dialog treten, ihr Professionswissen teilen und in der gemeinsamen Reflektion neue Perspektiven entwickeln, kommt wesentlicher seltener vor (Böttcher et al., 2011; Fussangel, 2008; Richter \& Pant, 2016). In der Konsequenz bleiben die Aufgabenbereiche von Lehrkräften und weiterem pädagogischen Personal häufig unverbunden, indem die eine Gruppe für den unterrichtlichen und die andere für den außerunterrichtlichen Bereich zuständig bleibt (Böttcher et al., 2011; Steiner \& Tillmann, 2011). Beim gemeinsamen Unterrichten von Regellehrkräften und sonderpädagogischen Lehrkräften ist eine solche Trennlinie schwieriger zu ziehen und insbesondere über die Rolle der sonderpädagogischen Lehrkräfte scheint teilweise eine Unklarheit zu herrschen, die eine effektive und für beide Seiten befriedigende Zusammenarbeit erschwert (Brähler, 1991; Gebhard et al., 2014; Lütje-Klose, 2011). Insgesamt deutet die Befundlage darauf hin, dass das Potential, das multiprofessioneller Kooperation für Innovationsprozesse an der Schule zugeschrieben wird, häufig ungenutzt bleibt.

Für die Untersuchung der Kooperation von Lehrkräften und Schulbegleiter/innen können aus den Ergebnissen zu schulischer Kooperation wichtige Ableitungen vorgenommen werden. Zum einen zeigen die Befunde, dass die Mehrheit der Lehrkräfte Kooperation für relevant hält (Esslinger, 2002; Richter \& Pant, 2016) und die Ausprägung kooperationserschwerender Merkmale wie ein hohes Autonomiebedürfnis nicht in überhöhtem Maße zu erwarten ist (Pröbstel \& Soltau, 2012). Die hohe Bedeutung individueller Merkmale für Kooperation (OECD, 2014; Pröbstel \& Soltau, 2012) und die auf Personenebene unterschiedliche Ausgestaltung von Kooperationsbeziehungen (Speck et al., 2011b) lassen erwarten, dass unabhängig von schulstrukturellen Rahmenbedingungen verschiedene Konstellationen der Zusammenarbeit von Lehrkräften mit Schulbegleiter/innen zu finden sind. Dabei kann aufgrund der vorgestellten Studien aber nach wie vor nicht davon ausgegangen werden, dass Kooperation selbstverständlicher Teil der Lehrerarbeit ist, so dass vielen Lehrkräften Erfahrungen in diesem Bereich fehlen, was Unsicherheiten befördern kann (Ahlgrimm, 2012). Als problematisch kann sich in diesem Zusammenhang auch der Mangel an festgelegten Strukturen zur institutionellen Absicherung von Kooperation erweisen. Schulbegleiter/innen arbeiten demnach in einem Umfeld, das nicht auf Kooperation ausgelegt ist, mit Lehrkräften, die möglicherweise über wenig ,interprofessionelle[n] Kompetenz" (Steiner \& Tillmann, 2011, S. 51) verfügen. Es muss deshalb damit gerechnet werden, dass das Konfliktpotential, das im Zusammenhang mit multiprofessioneller Kooperation theoretisch und empirisch beschrieben wird, auch in der Zusammenarbeit von Lehrkräften und Schulbegleiter/innen vorhanden ist. Unterschiede im beruflichen Status, im Verständnis der eigenen Rolle und der Auffassung von Erziehung, die zwischen Lehrkräften und weiterem pädagogisch tätigen Personal bzw. Regelschulehrkräften und sonderpädagogischen Lehrkräfte bestehen (Arndt et al., 2014; Breuer, 2011; Hedderich \& Hegner, 2009; Hillenbrand, 2012; Speck et al., 2011a, 2011b), können auch in der Kooperationsbeziehung von Lehrkräften und Schulbegleiter/innen eine Rolle spielen. Schulbegleiter/innen sind keine ausgebildeten Lehrkräfte und vom Qualifikationshintergrund vermutlich ähnlich heterogen wie das weitere pädagogisch tätige Personal. Ihr Professionsverständnis als Eingliederungshelfer/innen ist möglicherweise dem von Schulsozialarbeiter/innen ähnlicher als dem von Regellehrkräften. Die schülerbezogene Zusammenarbeit im Unterricht als dem Ort, an dem nach wie vor wenig Kooperation stattfindet (Böttcher et al., 2011; Fussangel, 2008; Steiner \& Tillmann, 2011; Steinert et al., 2006), birgt wahrscheinlich nicht nur für Regellehrkräfte und sonderpädagogische Lehrkräfte Herausforderungen, sondern auch für Regellehrkräfte und Schulbegleiter/innen, zumal in beiden Fällen eine Art doppelter Zuständigkeit für bestimmte Schüler/innen vorhanden ist. Zuletzt kann aus den theoretischen und empirischen Analysen zu Kooperation im Schulbereich die Bedeutsamkeit der Unterscheidung verschiedener Kooperationsformen abgeleitet werden. Nicht jede Form von Interaktion zwischen zwei beruflich an der Schule tätigen Personen ist arbeitsbezogen und 
innerhalb dessen, was an arbeitsbezogener Kooperation stattfindet, können verschiedene Ausprägungsgrade von Komplexität und Anspruch ausgemacht werden (z. B. Fussangel, 2008). So ist etwa zu vermuten, dass bei einfachen Kooperationsformen zwischen Lehrkräften und Schulbegleiter/innen keine gegenseitige fachliche Bereicherung durch die Kooperation stattfindet, dafür das Konfliktpotential aber geringer ausfällt (Fussangel, 2008). Im Fall anspruchsvoller angelegter Kooperation kann dagegen erwartet werden, dass von Schulbegleiter/innen als möglicher „pädagogischer Laien“ (Steiner, 2013, S. 64) durchaus Impulse für Innovations- und Professionalisierungsprozesse an Schulen ausgehen können (ebd.).

Die angestellten Überlegungen zeigen, dass sich die Kooperation von Lehrkräften und Schulbegleiter/innen in die bisherige Forschung über Kooperation an Schulen sinnvoll einbetten lässt. Insbesondere die Parallelen zum weiteren pädagogisch tätigen Personal und die Nähe zum Einsatzgebiet der Sonderpädagogik bieten viele Anknüpfungspunkte. Daneben lassen sich aus der Diskussion um die Maßnahme Schulbegleitung und aus der empirischen Befundlage Besonderheiten ableiten, die Schulbegleiter/innen von anderen Berufsgruppen unterscheiden. Anders als das weitere pädagogisch tätige Personal ist Schulbegleitung primär im Unterricht tätig, so dass die Arbeit von Lehrkräften und Schulbegleiter/innen weder räumlich noch zeitlich getrennt sein kann und Kooperation in irgendeiner Form unumgänglich wird. Anders als die sonderpädagogischen Lehrkräfte gehören Schulbegleiter/innen keiner Profession an, die über einheitliche Qualifizierungsstandards definiert ist. Aufgrund der mangelnden Konzeptualisierung des Anforderungs- und Aufgabenprofils von Schulbegleitung ist denkbar, dass die Frage der eigenen Rolle für Schulbegleiter/innen von noch größerer Bedeutung ist als für sonderpädagogische Lehrkräfte: Schulbegleiter/innen durchlaufen keine formale Ausbildung, die sie auf ihre spezielle Tätigkeit vorbereitet, so dass die Bestimmung des eigenen Aufgabenbereichs und die Ausgestaltung der eigenen Rolle im Wesentlichen während der Durchführung der Maßnahme geschieht, d. h. im Unterrichtskontext. Die meiste Arbeitszeit teilen sie mit der Klassenleitung, die auch als Zuständige für den Schüler oder die Schülerin von zentraler Wichtigkeit ist. Es ist deshalb zu erwarten, dass im Fall der Kooperation von Schulbegleitung und Klassenleitung in besonderem Maße gilt, dass die Grenzen von Zuständigkeitsbereichen ,, nur über Absprache unter den Beteiligten in der jeweiligen Situation abgesteckt werden“ (Rahm et al., 2015, S. 146) können. Eine Besonderheit der Kooperation von Schulbegleitung und Klassenleitung liegt auch in der ausgeprägten Statusungleichheit. Zum einen sind die Klassenleitungen häufig für die Einarbeitung der Schulbegleiter/innen zuständig (zumindest im Göttinger Raum) und tragen in jedem Fall die Verantwortung für die gesamte Klasse einschließlich der begleiteten Schüler/innen; sie sind Schulbegleiter/innen von daher im Arbeitskontext übergeordnet. Zum anderen arbeiten Schulbegleiter/innen - im Gegensatz zu Lehrkräften - in teilweise prekär zu nennenden Beschäftigungsverhältnissen (Heinrich \& Lübeck, 2013) und in vielen Fällen ohne einschlägige Qualifikation.

Theoretisch liegt es nahe zu vermuten, dass aus Schulbegleitersicht die Klärung der eigenen Rolle und die Wahrnehmung von Wertschätzung wichtige Aspekte bei der Kooperation mit der Klassenleitung darstellen. Diese Annahme lässt sich auch empirisch aus den Ergebnissen qualitativer Diskussionsdokumentationen (Deger et al., 2015; Lindmeier et al., 2014) und der Fallrekonstruktion von Lübeck \& Heinrich (2016) ableiten und wird durch Befunde der internationalen Forschung zu nichtprofessionellem Assistenzpersonal gestützt (Blatchford et al., 2009a, 2009b; Butt \& Lowe, 2012; Fisher \& Pleasants, 2012; Hanover Research, 2010; Köpfer, 2013a, 2013b; Webster et al., 2010, 2011). Rollensicherbeit wird in Anlehnung an Edmonson (2006) im Folgenden spezifiziert als der Grad an Gewissheit über die Zuständigkeiten und Erwartungen, die im beruflichen Kontext an die eigene Person gestellt werden, und die damit zusammenhängenden Aufgaben sowie die Ziele ihrer Arbeit. Die Erfahrung von Wertschätrung wird im Folgenden als Erfahrung arbeitsbezogener Wertschätzung verstanden, d. h. der Erfahrung von Anerkennung und Respekt der eigenen professionellen Kompetenzen. Auf Basis der Befundlage zu Kooperation an Schulen ist davon auszugehen, dass Kooperation mit der Klassenleitung auch zum Erleben emotionaler Entlastung führen kann, indem sie hilft, realistische Ansprüche an die eigene Arbeit zu entwickeln, und soziale Unterstützung bei der Bewältigung arbeitsbezogener Herausforderungen bietet (Böhm-Kasper, 2004; Schaarschmidt, 2005). Emotionale Entlastung wird im Folgenden verstanden als Erleichterung von als negativ empfundenen Gefühlszuständen, die im Zuge der Arbeitsausführung auftreten. Dagegen ist nicht zu erwarten, dass Schulbegleiter/innen infolge der Kooperation mit der Klassenleitung eine bedeutsame Arbeitsentlastung erfahren; die Abgabe von Arbeitsaufgaben und Zuständigkeiten ist wenn, dann eher von der Klassenleitung an die Schulbegleitung möglich. Als weiterer wichtiger Aspekt bei der Kooperation mit der Klassenleitung kann die Arbeitszufriedenheit gesehen werden, die im Folgenden als positive Einstellung einer Person gegenüber ihrer Arbeitstätigkeit verstanden wird. Für die Bewältigung der Herausforderungen, vor die Schulbegleiter/innen im Rahmen ihrer Arbeit gestellt werden, ist die Kooperation mit der Klassenleitung ein entscheidender Faktor, 
oder anders herum formuliert: Wenn die Kooperation mit der Klassenleitung nicht gelingt, ist schwer vorstellbar, wie die Arbeit als Schulbegleitung angesichts der Unumgänglichkeit der Zusammenarbeit im Unterricht und der gleichzeitigen Zuständigkeit für dasselbe Kind als zufriedenstellend erlebt werden kann (vgl. hierzu die Ergebnisse von Gebhard et al., 2014, zur Zufriedenheit von kooperierenden sonderpädagogischen Lehrkräften).

Aufbauend auf den Erkenntnissen aus der Lehrerkooperationsforschung ist für die Kooperation von Schulbegleitung und Klassenleitung darüber hinaus anzunehmen, dass zwischen verschiedenen Kooperationsformen unterschieden werden kann, die sich im Grad ihrer Komplexität unterscheiden. Insbesondere das Modell von Gräsel et al. (2006) bzw. Fussangel (2008) erlaubt eine Adaption für die Untersuchung der Kooperation von Schulbegleitung und Klassenleitung. Gegenüber anderen Modellen zeichnet es sich durch seine differenzierte Beschreibung von unterschiedlich elaborierten Kooperationsformen auf Ebene der konkret ausgeführten Tätigkeiten aus. Weitere Vorteile des Modells sind seine empirische Fundierung und die breite Anwendung in nachfolgenden Studien zu Kooperation, die den Vergleich und die Einordnung der Ergebnisse zur Kooperation mit Schulbegleiter/innen in die bisherige Kooperationsforschung erleichtern. In adaptierter Form wurde das Modell bereits zur Untersuchung multiprofessioneller Kooperation genutzt (Dizinger \& Böhm-Kasper, 2012), allerdings in einer Form, die sich schwieriger auf die Kooperation mit Schulbegleiter/innen übertragen lässt als das ursprüngliche Modell. Im Folgenden wird die eigene Adaption des Modells beschrieben. Bei der Kooperation von Schulbegleitung und Lehrkraft lassen sich theoretisch zwei Formen von Kooperation annehmen. Auf einer wenig komplexen Stufe lässt sich ihre Kooperation beschreiben als Koordination. Auf dieser Stufe finden Austausch und Absprachen über den betreffenden Schüler oder die betreffende Schülerin statt. Beide Seiten versorgen sich gegenseitig mit wichtigen arbeitsbezogenen Informationen (vgl. hierzu die Beschreibung der Kooperationsform Austausch von Fussangel, 2008) und treffen Absprachen hinsichtlich der Arbeit der Schulbegleitung. Die Autonomie beider Seiten bleibt dabei weitgehend gewahrt, insbesondere aber die der Klassenleitung, da sich die Koordination im Wesentlichen auf die Arbeit der Schulbegleitung bezieht. Eine Verständigung über die jeweiligen Perspektiven auf den Schüler oder die Schülerin und über gemeinsame Ziele ist auf dieser Stufe nur eingeschränkt nötig. Auf einer komplexeren Stufe lässt sich die Kooperation zwischen Schulbegleitung und Klassenleitung in Anlehnung an Gräsel et al. (2006) und Fussangel (2008) als Kokonstruktion beschreiben. Auf dieser Stufe findet eine gemeinsame Reflektion über die Arbeit sowohl der Schulbegleitung als auch der Klassenleitung statt. Beide Seiten erhalten auf diese Weise Rückmeldung zu ihrer eigenen Arbeit, teilen ihr Wissen und ihre Perspektiven auf den Schüler oder die Schülerin und entwickeln daraus gemeinsame Problemlösungen. Kooperation in diesem Sinne realisiert in stärkerem Maße die Bedingungen von professioneller Kooperation, wie sie insbesondere in der arbeitspsychologischen Definition von Spieß (2004) formuliert sind (vgl. Fussangel, 2008); sie setzen ein höheres $\mathrm{Maß}$ an Vertrauen voraus und beinhalten eine stärkere gemeinsame Zielbindung. Zugleich können von dieser Kooperationsform stärkere Impulse zur Professionalisierung der Schulbegleitung und der Klassenleitung ausgehen, indem beide Seiten angehalten sind, ihr Rollenverständnis und ihre professionellen Kompetenzen weiterzuentwickeln. Wenn Kooperation, wie angenommen, mit emotionaler Entlastung, Wertschätzung, Rollensicherheit und Arbeitszufriedenheit zusammenhängt, kann weiterhin angenommen werden, dass dieser Zusammenhang bei Kokonstruktion stärker ausfällt als bei Koordination (siehe Abbildung 2), da sie die vergleichsweise intensivere Kooperationsform darstellt.

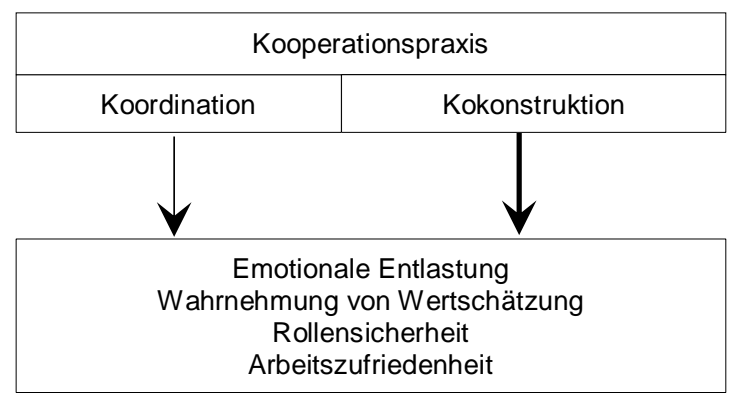

Abb. 2: Arbeitsmodell zur Kooperation von Klassenleitung und Scbulbegleitung 
Im Zusammenhang mit der Untersuchung der Kooperation von Schulbegleitung und Klassenleitung scheint als zusätzlicher Aspekt die Erfassung der berufsbezogenen Selbstwirksamkeitserwartung von Schulbegleiter/innen interessant, insbesondere vor dem Hintergrund, dass Schulbegleiter/innen keine einschlägige Ausbildung für ihre Tätigkeit absolviert haben. Verschiedene Studien zeigen für Lehrkräfte einen Zusammenhang zwischen dem beruflichen Kompetenzerleben und der Ausprägung von Kooperation in einfacher wie komplexerer Form (Dizinger et al., 2011a; OECD, 2014; Richter \& Pant, 2016). Allgemein bezeichnet Selbstwirksamkeitserwartung die Erwartung, ,ob man sich selbst in der Lage sieht, ein Verhalten zu zeigen, das zu einem Erfolg führt“ (Köller \& Möller, 2006, S. 693) bzw. „die subjektive Gewissheit, neue oder schwierige Anforderungssituationen aufgrund eigener Kompetenz bewältigen zu können“ (Schwarzer \& Warner, 2014, S. 662). Entsprechend bezieht sich die berufsbezogene Selbstwirksamkeitserwartung auf die Einschätzung der eigenen Fähigkeiten, arbeitsbezogene Herausforderungen erfolgreich zu bewältigen. Empirisch ist sie nicht nur positiv mit Kooperation (Dizinger et al., 2011a, 2011b), sondern auch positiv mit Arbeitszufriedenheit und negativ mit beruflichem Belastungserleben assoziiert (Böhm-Kasper et al., 2009; Schmitz \& Schwarzer, 2000).

Zusammenfassend kann festgestellt werden, dass weiterer Forschungsbedarf sowohl hinsichtlich der Erfassung lokaler Rahmendaten zur Maßnahme Schulbegleitung als auch hinsichtlich der systematischen Untersuchung der Kooperation mit Schulbegleiter/innen besteht. Aus dem Mangel an theoretisch fundierten Analysen der Kooperationspraxis ergibt sich auch, dass keine Studien vorliegen, die eine Verknüpfung von kooperationsspezifischen Aspekten mit Merkmalen von Schulbegleiter/innen und allgemeinen Daten zur Maßnahme vornehmen. Ein Teil dieser Forschungslücke soll durch die Untersuchung bearbeitet werden, die im nun anschließenden empirischen Teil dieser Arbeit beschrieben wird. 


\section{Forschungsfragen und Hypothesen}

Aus den im theoretischen Teil dieser Arbeit herausgearbeiteten Forschungsdesideraten und den vorgenommenen Ableitungen aus bisherigen theoretischen wie empirischen Befunden zu Schulbegleitung und zu Kooperation an Schulen gehen zwei übergeordnete Fragestellungen für die eigene Untersuchung hervor, die im Folgenden näher ausgeführt werden.

\subsection{Rahmendaten zu Schulbegleitung in Stadt und Landkreis Göttingen}

Die erste übergeordnete Fragestellung lautet: Wie lässt sich die Maßnahme Schulbegleitung in Stadt und Landkreis Göttingen beschreiben? Aufgrund der deskriptiven Anlage dieser Fragestellung werden hierzu keine Hypothesen gebildet, sondern Forschungsfragen hinsichtlich der lokalen Schulbegleitungssituation gestellt:

- Wie lassen sich Person und Qualifikation von Schulbegleiter/innen beschreiben? Welche formalen Qualifikationen, welche Vorerfahrungen und Motive bringen sie mit? Wie lange sind sie bereits als Schulbegleitung tätig und wie lange möchten sie noch als Schulbegleitung tätig sein?

- Wie lässt sich das Beschäftigungsverbältnis, in dem Schulbegleiter/innen stehen, beschreiben? Welches Amt ist Kostenträger, wer tritt als Arbeitgeber auf? Wie sind die Vertragslaufzeiten und wie bewerten die Schulbegleiter/innen einzelne Aspekte des Arbeitsvertrags? Bietet der Arbeitgeber Schulungen an?

- Wie lassen sich die begleiteten Schüler/innen beschreiben? Welche Behinderung liegt der Beantragung zu Grunde und in welchem Umfang erhalten sie Schulbegleitung? Welche Schulform besuchen sie und wie ist der Unterricht gestaltet, in dem sie begleitet werden?

- Wie lässt sich das Tätigkeitsprofil von Schulbegleiter/innen beschreiben? Zu welchen Anteilen werden Aufgaben der Bereiche Unterstützung bei lebenspraktischen Anforderungen, Unterstützung bei der Emotions- und Verhaltensregulation und didaktische Unterstützung ausgeführt?

Darüber hinaus interessiert, in welchen Zusammenhängen die genannten Aspekte zueinander stehen. Auch in diesem Rahmen wird auf die Formulierung von Hypothesen verzichtet und vor dem Hintergrund der Ausführungen im theoretischen Teil werden stattdessen folgende explorative Forschungsfragen gestellt:

- In welchem Zusammenhang steht die Qualifikation der Schulbegleiter/innen mit

- den Motiven zur Aufnahme der Beschäftigung als Schulbegleitung, 
- der Dauer, wie lange man noch als Schulbegleitung tätig sein möchte, und

- mit der Bewertung des Beschäftigungsverhältnisses?

- In welchem Zusammenhang stehen die Art der Behinderung bzw. das bewilligende Amt mit der besuchten Schulform?

- In welchem Zusammenhang stehen die Art der Behinderung bzw. das bewilligende Amt und die besuchte Schulform jeweils mit

- dem Bewilligungszeitraum der Maßnahme,

- den Tätigkeiten, die Schulbegleiter/innen ausführen, und

- der Qualifikation von Schulbegleiter/innen?

- In welchem Zusammenhang stehen Unterrichtsformen und die Tätigkeiten, die Schulbegleiter/innen ausführen?

\subsection{Kooperation mit Schulbegleiter/innen}

Die zweite übergeordnete Fragestellung lautet: Wie lässt sich die Einbindung von und Kooperation mit Schulbegleiter/innen in der Schule beschreiben? Zur Erfassung der allgemeinen Kooperationssituation werden folgende deskriptiv bzw. explorativ angelegte Forschungsfragen zu Häufigkeit und Bewertung von Austausch formuliert:

- Mit welchen Personengruppen stehen Schulbegleiter/innen wie häufig in Austausch?

- Wie bewerten Schulbegleiter/innen die Wichtigkeit des Austauschs und ihre eigene Zufriedenheit des Austauschs mit verschiedenen Personengruppen?

- Sind die Personengruppen, mit denen der häufigste Austausch stattfindet, auch diejenigen, mit denen der Austausch für am wichtigsten gehalten wird?

Zur Kooperation von Klassenleitung und Schulbegleiter/innen können auf Basis theoretischer Modelle und empirischer Befunde in der Forschung zu Kooperation und zu Schulbegleitung bzw. Assistenzpersonal spezifische Hypothesen formuliert werden. Es lässt sich annehmen, dass die Ausprägung der Kooperation mit der Klassenleitung positive Auswirkungen auf das Erleben von Schulbegleiter/innen hat und zwar hinsichtlich emotionaler Entlastung, der wahrgenommenen Wertschätzung und Rollensicherheit sowie der Arbeitszufriedenheit. Weiterhin lässt sich annehmen, dass die Kooperationspraxis von Klassenleitung und Schulbegleitung unterschiedlich anspruchsvoll ausgestaltet sein kann und weniger anspruchsvolle Kooperationstätigkeiten dominieren. Man kann zudem davon ausgehen, dass anspruchsvoller angelegte Kooperation in höherem Maße gegenseitiges Vertrauen, den Austausch und die gemeinsame Reflektion professionellen Wissen und Handelns, die Verständigung über das gegenseitige Rollenverständnis und die Aushandlung für beide Seiten befriedigender gemeinsamer Arbeitsziele beinhaltet (vgl. Fussangel, 2008; Kuper \& Kapelle, 2012; Speck et al., 2011a, 2011b; Spieß, 2004). Aus diesem Grund kann erwartet werden, dass von der Ausprägung anspruchsvollerer Kooperationspraxis stärkere Wirkungen auf die emotionale Entlastung, die Wahrnehmung von Wertschätzung, die Rollensicherheit und die Arbeitszufriedenheit von Schulbegleiter/innen ausgehen als von der Ausprägung einer weniger anspruchsvollen Kooperationspraxis (vgl. ebd.). Diese Hypothesen sollen im Rahmen des in Kapitel 4 vorgestellten Arbeitsmodells zur Kooperation von Schulbegleitung und Lehrkraft untersucht werden. Die Hypothesen zur Kooperationspraxis mit der Klassenleitung und Kooperationswirkungen lauten:

- Einfache Kooperation im Sinne von Koordination ist stärker ausgeprägt als komplexe Kooperation im Sinne von Kokonstruktion. (Gerichtete Unterschiedshypothese)

- Die Ausprägung von Koordination steht in positivem Zusammenhang zur Ausprägung von (a) emotionaler Entlastung, (b) Wertschätzung, (c) Rollensicherheit und (d) Arbeitszufriedenheit von Schulbegleiter/innen. (Gerichtete Zusammenhangshypothese)

- Die Ausprägung von Kokonstruktion steht in positivem Zusammenhang zur Ausprägung von (a) emotionaler Entlastung, (b) Wertschätzung, (c) Rollensicherheit und (d) Arbeitszufriedenheit von Schulbegleiter/innen (Gerichtete Zusammenhangshypothese) 
- Die Ausprägung von Kokonstruktion steht in stärkerem Zusammenhang zur Ausprägung von (a) emotionaler Entlastung, (b) Wertschätzung, (c) Rollensicherheit und (d) Arbeitszufriedenheit von Schulbegleiter/innen als die Ausprägung von Koordination. (Gerichtete Unterschiedshypothese)

Vor dem Hintergrund bisheriger Forschungsergebnisse lassen sich Zusammenhänge zwischen der Ausprägung der Kooperation und der hierfür aufgewendeten Zeit und der Bewertung des Austauschs annehmen (Ahlgrimm, 2012; Böttcher et al., 2011), jedoch lässt die Befundlage keine klare Ableitung von Hypothesen $\mathrm{zu}$, so dass anstelle von Hypothesen folgende explorative Forschungsfragen gestellt werden:

- In welchem Zusammenhang steht die Ausprägung von Koordination und Kokonstruktion mit der Häufigkeit des Austauschs mit der Klassenleitung, der Wichtigkeit, die die Schulbegleitung dem Austausch beimisst, und die Zufriedenheit der Schulbegleitung mit diesem Austausch?

Zur Kooperation mit der Klassenleitung wird auf Basis empirischer Befunde zum Zusammenhang von Kooperation und dem Erleben beruflicher Zufriedenheit mit dem Erleben eigener beruflicher Kompetenz außerdem folgende Forschungsfrage gestellt:

- In welchem Zusammenhang steht arbeits- bzw. berufsbezogene Selbstwirksamkeitserwartung mit der Ausprägung von Koordination und Kokonstruktion sowie der emotionalen Entlastung, Wertschätzung, Rollensicherheit und Arbeitszufriedenheit von Schulbegleiter/innen?

Zuletzt interessiert, wie Schulbegleiter/innen die Einbindung an der Schule insgesamt bewerten. Zur Einbindung in die Schulgemeinschaft und Gesamtzufriedenheit werden folgende deskriptive angelegte Forschungsfragen gestellt:

- Inwieweit fühlen sich die Schulbegleiter/innen in die Schulgemeinschaft eingebunden?

- Wie zufrieden sind die Schulbegleiter/innen mit der Einbindung und Zusammenarbeit an der Schule?

- Welche Gründe sind maßgeblich für die Zufriedenheit der Schulbegleiter/innen mit der Einbindung und Zusammenarbeit an der Schule?

Zum Zusammenhang der Rahmendaten zu Schulbegleitung und der Variablen zu Einbindung und Kooperation lassen sich aus der bisherigen Befundlage keine klaren Hypothesen ableiten. Vor dem Hintergrund der Ergebnisse der StEG-Studie zur Kooperation und Einbindung hinsichtlich des weiteren pädagogisch tätigen Personals (Dieckmann et al., 2008; Steiner \& Tillmann, 2011; Tillmann \& Rollett, 2011) stellen sich aber folgende Forschungsfragen zu Rahmendaten und Kooperationsvariablen:

- Welche Zusammenhänge bestehen jeweils zwischen der Ausprägung von Koordination und Kokonstruktion mit

- der formalen Qualifikation der Schulbegleiter/innen,

- ihrer bisherigen Tätigkeitsdauer,

- der Schulform,

- den von ihnen ausgeführten Tätigkeiten und

- der Einbindung in die Schulgemeinschaft?

- Besteht ein Zusammenhang zwischen den Schulformen und der Einbindung in die Schulgemeinschaft? 


\section{Datengrundlage und methodisches Vorgehen}

Im Folgenden wird zunächst das Studiendesign erläutert und daraufhin die Stichprobe und die Durchführung der Datenerhebung. Anschließend wird die Entwicklung des eingesetzten Fragebogens beschrieben sowie einzelne Inhalte und Items des Fragebogens und abschließend das Vorgehen bei der Datenanalyse.

\subsection{Studiendesign, Stichprobe und Datenerhebung}

Die vorliegende Untersuchung wurde als vollstandardisierte Fragebogenstudie (Zierer, Speck \& Moschner, 2013) konzipiert, mit der im Querschnitt Angaben von Schulbegleiter/innen zu den in Kapitel 5 näher erläuterten Themengebieten erfasst werden. Zur Untersuchung der übergeordneten Fragestellungen (Rahmendaten zur Beschreibung von Schulbegleitung in Stadt und Landkreis Göttingen sowie der Einbindung und Kooperation mit Schulbegleitungen) wäre eine multiperspektivische Erhebung denkbar gewesen. Insbesondere zur Erfassung der Kooperation zwischen Schulbegleiter/innen und Lehrkräften wäre eine Befragung beider Personengruppen wünschenswert gewesen, was im Rahmen dieser Arbeit jedoch nicht möglich war. Aus pragmatischen Gründen wurden deshalb nur Schulbegleiter/innen befragt. Die Anlage der vorliegenden Untersuchung lässt sich als überwiegend explorativ bezeichnen, nur zum Teil dient sie der Überprüfung von Hypothesen. Der explorativen Anlage entsprechend werden an verschiedenen Stellen (halb)offene Abfragen vorgenommen.

Die Stichprobe umfasst nach Bereinigung um vorzeitige Abbrecher/innen 47 Schulbegleiter/innen aus Stadt und Landkreis Göttingen. Da sie Teil der Forschungsfragen sind, werden die demographischen Angaben zur Stichprobe im Ergebnisteil berichtet. Die Befragung wurde online mit der Befragungssoftware Unipark (QuestBack) durchgeführt, da die Kontaktaufnahme per Mail den einfachsten Zugang zur Zielgruppe darstellte. ${ }^{23}$ Die erste Erhebung erfolgte im Sommer 2015. Um alle Schulbegleiter/innen in Stadt und Landkreis Göttingen zu erreichen, wurde im Vorhinein mit allen zuständigen Trägern und Ämtern telefonischer Kontakt aufgenommen. Sämtliche Träger verfügten über Verteiler mit den Emailadressen der bei ihnen beschäftigten Schulbegleiter/innen. Die Schulbegleiter/innen, die über das Persönliche Budget von den Eltern des Kindes selbst angestellt wurden, ließen sich weder über die Träger noch über die Ämter erreichen. Da diese Gruppe nach Auskunft der Ämter zahlenmäßig nicht ins Gewicht fällt, wird ihr Fehlen in der Stichprobe im Folgenden nicht mehr gesondert erwähnt. Die Zuständigen aller Träger sowie des Jugendamts Stadt (das als einziges Amt eine eigene Kontaktliste führt, die sich aber mit denen der Träger doppelt)

23 Parallel wurden Printversionen des Fragebogens (inklusive vorfrankierter Rückumschläge) auf dem Sommerfest des zahlenmäBig wichtigsten Trägers von Schulbegleitung verteilt. Von den dort verteilten Bögen wurden vier zurückgesandt. 
erklärten sich im Vorhinein bereit, meine Email mit dem Anschreiben und dem Link zur Umfrage weiterzuleiten. Auf diese Weise wurde sichergestellt, dass keine privaten Daten herausgegeben wurden. ${ }^{24}$

Das Anschreiben in der Email enthielt neben einer persönlichen Ansprache zum einen Informationen über den Hintergrund, die Inhalte und die Ziele der Studie und zum anderen Hinweise zur Bearbeitungsdauer (etwa 30 Minuten), zur Teilnahmefrist und zum Datenschutz. Es wurden zugesichert, dass - außer bei freiwilliger Angabe von Kontaktdaten ${ }^{25}$ - keine Daten erhoben würden, die Rückschlüsse auf die Person zulassen würden ${ }^{26}$ und dass alle Daten streng vertraulich behandelt und nicht an Dritte weitergegeben würden. Zusätzlich wurde erklärt, dass Ergebnisdarstellungen nur in Form von Häufigkeiten oder Durchschnittswerten der Antworten aller Teilnehmenden erfolgen würden. Zuletzt enthielt das Anschreiben meine Kontaktdaten und den Hinweis auf die Möglichkeit zur Teilnahme an einem Gewinnspiel nach Beendigung der Umfrage. Als Kontaktdaten wurden meine persönliche Emailadresse der Universität und die Postadresse der Institutsabteilung angegeben. Die universitäre Einbettung der Untersuchung wurde zusätzlich hervorgehoben durch die Einbindung des Universitäts-Logos in den Fragebogen (siehe hierzu Bortz \& Döring, 2006). Die Startseite der Unipark-Umfrage beinhaltete ebenfalls ein Anschreiben, das kurz die wichtigsten Punkte aus dem Emailanschreiben (insbesondere zu Datenschutz, Bearbeitungszeit, Kontaktdaten) sowie Bearbeitungshinweise - unter anderem den Hinweis, dass bei Einschätzungsfragen die eigene ehrliche Einschätzung interessiert und es keine richtigen oder falschen Antworten gibt (vgl. Bühner, 2011) - enthielt.

Vom 07. Juli bis zum 09. August 2015 konnten die Schulbegleiter/innen an der Befragung teilnehmen. Die Fristsetzung etwa einen Monat nach Beginn der Umfrage sollte einerseits ausreichend Zeit zur Teilnahme geben, andererseits zur zeitnahen Teilnahme motivieren um ein Versäumnis zu verhindern. Der Zeitraum wurde so ausgewählt, dass alle Schulbegleiter/innen am Ende des Schuljahres, aber noch vor den Sommerferien erreicht wurden. Etwa zwei Wochen vor Fristende wurden die Träger gebeten, eine E-Mail zur Erinnerung an das Fristende weiterzuleiten. Im genannten Erhebungszeitraum beteiligten sich 42 Personen an der Umfrage, von denen zwei etwa nach der Hälfte des Fragebogens die weitere Beantwortung abbrachen. Nicht mitgezählt sind 16 weitere Teilnehmende, die keine oder nur die ersten vier Fragen beantwortet hatten. Die Höhe der Rücklaufquote lässt sich anhand der Angaben der Träger schätzen. Demnach beschäftigten die Träger im Juli 2015 etwa 160 Schulbegleiter/innen in Stadt und Landkreis Göttingen, so dass sich eine Rücklaufquote von 27\% ergibt. Der Anteil der Stichprobe an der Grundgesamtheit an Schulbegleiter/innen inklusive derer, die nicht bei freien Trägern arbeiten, ist nicht eindeutig zu bestimmen, da von einigen Ämtern nur die Fallzahlen zum Jahresende zu ermitteln waren. ${ }^{27}$

Eine Rücklaufquote von 27\% kann als zufriedenstellend angesehen werden (vgl. Kißgen et al., 2013), um aber eine höhere Ausschöpfung zu erreichen, wurde im Dezember des gleichen Jahres eine Nacherhebung durchgeführt. Auf diese Weise sollten auch die in der Zwischenzeit neu eingestellten Schulbegleiter/innen erreicht werden. Die Kontaktierung und der Ablauf der Erhebung erfolgten auf dieselbe Weise wie bei der ersten Erhebung. ${ }^{28}$ Die Teilnahme war vom 06. Dezember 2015 bis zum 04. Januar 2016 möglich. In diesem Zeitraum nahmen fünf Personen an der Umfrage teil (keine vorzeitigen Abbrüche). ${ }^{29} \mathrm{Da}$ die Gesamtpopulation beider Zeitpunkte verschieden sein kann ${ }^{30}$, ist eine Berechnung der Ausschöpfungsquote aller

24 Ein Träger besaß von einigen seiner Schulbegleiter/innen nur die Postadresse und verschickte in meinem Namen das Anschreiben mit dem Link zur Umfrage auf dem Postweg.

25 Im Fall einer sehr geringen Beteiligung an der Umfrage (der nicht eingetreten ist) sollten zusätzlich Interviews mit ausgewählten Schulbegleiter/innen geführt werden. Zu diesem Zweck war bei der ersten Erhebung die Frage nach der Bereitschaft zur Interviewteilnahme und der Eingabe von Kontaktdaten am Ende des Fragebogens eingefügt.

26 Aus diesem Grund wurde zur Teilnahme am Gewinnspiel (Einkaufsgutscheine für Göttinger Innenstadtgeschäfte) eine zweite Umfrage in Unipark erstellt, zu der am Ende des Fragebogens verlinkt wurde. Die Kontaktdaten zur Teilnahme am Gewinnspiel waren auf diese Weise nicht den Angaben in der Umfrage zuordenbar.

27 Die amtliche Gesamtzahl laufender Fälle betrug zum Jahresende 215. Zu vermuten ist, dass die Differenz zu den Angaben der Träger für Juli des gleichen Jahres im Wesentlichen dem Anstieg an Schulbegleitungen geschuldet ist. Im Jugendamt Stadt etwa erhöhte sich die Fallzahl von 69 im März auf 96 im Dezember 2015. Inwieweit die Differenz auch auf trägerungebunde, nur in der amtlichen Statistik erfasste Schulbegleitungen zurückzuführen ist, ließ sich nicht ermitteln.

28 Einzig die Bitte um Teilnahme im Anschreiben wurde neu formuliert (um sowohl den neu eingestellten Schulbegleiter/innen als auch denen, die die Einladung zur Teilnahme schon im Sommer erhalten hatten und ggf. schon teilgenommen hatten, zu entsprechen) und im Fragebogen eine geringfügige Änderung vorgenommen: Die Abfrage demographischer Daten wurde ans Ende gestellt, da bei der ersten Erhebung viele Abbrüche auf den ersten Seiten erfolgten.

29 Durch manuellen Datenvergleich wurde ausgeschlossen, dass es sich um Doppelteilnahmen von Teilnehmenden der ersten Erhebung handelte.

30 Den gemachten Angaben nach war nur eine Teilnehmerin der zweiten Erhebung im Juli 2015 noch nicht als Schulbegleitung tätig. Die Stichprobe wurde also nicht nennenswert um Neueingestellte vergrößert. Laut den Angaben, wie lange man noch als 
im Dezember tätigen Schulbegleiter/innen nicht möglich. Es lässt sich also lediglich festhalten, dass mit der Umfrage schätzungsweise fast ein Drittel aller Schulbegleiter/innen, die im Sommer 2015 in Stadt und Landkreis Göttingen über freie Träger tätig waren, erreicht wurde. Die Bearbeitungszeit lag im Schnitt bei 27 Minuten $^{31}$, was im Rahmen der geschätzten Bearbeitungsdauer lag.

\subsection{Erhebungsinstrument}

Der Fragebogen sollte einer zweifachen Zielsetzung gerecht werden, einerseits der Erfassung allgemeiner deskriptiver Daten zu Schulbegleitung in Stadt und Landkreis Göttingen und andererseits der Erfassung kooperationsspezifischer Variablen. Zum zweiten Themenkomplex liegen bislang keine Studien vor, auf deren Erhebungsinstrumente zurückgegriffen werden könnte. Für den ersten Themenkomplex konnte nur teilweise auf bisherige Studien zu Schulbegleitung bzw. zu weiterem pädagogisch tätigen Personal an Schulen zurückgegriffen werden. Der detaillierten Beschreibung beider Teile des eingesetzten Fragebogens steht deshalb eine zusammenfassende Erläuterung der Entwicklung voran. Für die genaue Formulierung der Fragen und Antwortmöglichkeiten sei auf den Anhang verwiesen.

\subsubsection{Entwicklung des Fragebogens}

Deskriptive Daten zu Schulbegleitung wurden bereits in mehreren Studien erfasst, kooperationsspezifische Variablen zu Schulbegleitung nur in Form einzelner, eher allgemein gehaltener Abfragen etwa zur Zufriedenheit mit der Zusammenarbeit mit der Klassenleitung. Zur Auswahl, Adaption und Ergänzung bereits vorhandener Items sowie zur Entwicklung neuer Items erfolgte deshalb eine umfangreiche Recherche. Grundlegend war hierbei die Auseinandersetzung mit dem Studium empirischer und nichtempirischer Literatur zu Schulbegleitung und zu (multiprofessioneller) Kooperation im Bildungsbereich. Zusätzlich wurden in teils mehrstündigen Gesprächen Informationen von einer Schulbegleiterin und weiteren Praxisvertreter/innen eingeholt (siehe Kapitel 2). Alle Items wurden anhand der Hinweise zur Itementwicklung nach Bühner (2011) entwickelt. Gemeinsam mit der Schulbegleiterin wurde der entwickelte Fragebogen abschlieBend auf Verständlichkeit und Angemessenheit hinsichtlich des Erlebens- und Erfahrungsbereich von Schulbegleiter/innen geprüft. Ein Pretest im eigentlichen Sinne war im Rahmen der vorliegenden Arbeit nicht möglich.

Im ersten Themenkomplex sollten hauptsächlich konkrete Sachverhalte erfasst werden, anhand derer sich die Population der Schulbegleiter/innen in Stadt und Landkreis Göttingen und wichtige Rahmendaten zur Maßnahme beschreiben lassen. Dafür konnte auf vorhandene Studien zurückgegriffen werden, in denen allgemeine deskriptive Daten von Schulbegleiter/innen erfasst wurden und in denen entweder detaillierte Ergebnisdarstellungen vorlagen (Dworschak, 2012c; Henn et al., 2014) oder der Fragebogen als Anhang verfügbar war (Keil et al., 2010; Wohlgemuth, 2009). Weitere Items wurden dem Fragebogen für weiteres pädagogisch tätiges Personal der StEG-Studie (Furthmüller, 2014) bzw. der IGLU-Studie (Bos et al., 2010) entnommen. Insgesamt konnten nur wenige Items unverändert aus vorhandenen Studien übernommen werden, der überwiegende Teil wurde adaptiert oder neu entwickelt, um die Untersuchung der formulierten Forschungsfragen zu Schulbegleitung zu ermöglichen.

Der zweite Themenkomplex, Einbindung und Kooperation, zielte neben der Erfassung konkreter Sachverhalte wie etwa der Häufigkeit von Austausch vor allem auf die Einschätzung und Bewertung der Kooperationspraxis und möglicher Wirkungen von Kooperation, wofür überwiegend Itembatterien zum Einsatz kamen. Die Gestaltung dieses Fragebogenteils gestaltete sich insofern schwieriger, als dass diese Themen in den bisherigen Studien zu Schulbegleitung kaum vorkamen und auf keine passenden Items zurückgegriffen werden konnte. Aus vorhandenen Studien zu (multiprofessioneller) Kooperation passten jeweils nur ein Teil der Items der entsprechenden Skalen auf die Kooperation zwischen Lehrkräften und Schulbegleiter/innen bzw. ließ sich sinnvoll adaptieren. Es konnten deshalb keine kompletten Itembatterien übernommen werden, viele Items wurden neu entwickelt oder in mehr oder weniger enger Anlehnung an bestehende Items der Studien von Fussangel (2008), Dizinger und Böhm-Kasper (2012) sowie der StEG-Studie (Furthmüller, 2014) und der IGLU-Studie (Bos et al., 2010) und mit Rückgriff auf die Teamcheckliste von Arndt

Schulbegleitung tätig sein möchte, waren dagegen 12 Schulbegleiter/innen der ersten Erhebung zur Zeit der zweiten Erhebung nicht mehr als Schulbegleitung tätig.

31 Die Werte der vorzeitigen Abbrecher/innen und ein Extremwert von 14.5 Stunden sind hierbei nicht eingerechnet. 
et al. (2014) formuliert. Einzig die berufsbezogene Selbstwirksamkeitsskala von Schmitz und Schwarzer (2000) konnte mit leichten Kürzungen und Änderungen übernommen werden.

Die Itembatterien wurden zusammengestellt mit dem Ziel, aus ihnen Skalenmittelwerte bilden zu können (Bortz \& Döring, 2006), d. h. die jeweils eine Batterie bildenden Items sollten möglich homogen, also einheitlich das interessierende Merkmal messen (Eindimensionalität) und verschiedene Ausprägungsgrade des Merkmals repräsentieren (ebd.). Obwohl erwartbar ist, dass bei neu entwickelten Tests eine datenbasierte Selektion von Items zur Skalenbildung notwendig wird, wurde aus ökonomischen Gründen die Anzahl der Items für jedes Merkmal mit vier bis sechs Items eher gering gehalten. Die Bearbeitungszeit sollte eine Länge von 30 Minuten nicht überschritten, um nicht hierdurch die Rücklaufquote der ohnehin begrenzten Population zu reduzieren (vgl. Bühner, 2011).

\subsubsection{Beschreibung der Fragebogenitems}

Da der Fragebogen gleichzeitig der Beschreibung von Schulbegleitung und der Untersuchung der Kooperationspraxis und -wirkungen dient, finden sich sowohl viele deskriptiv angelegte Items auf Nominalskalenniveau als auch Itembatterien, die auf Intervallskalenniveau Einschätzungen und Bewertungen erfassen. An dieser Stelle erfolgt eine gebündelte Übersicht über die verwendeten Antwortformate, um unnötige Wiederholungen bei der Beschreibung der Einzelitems zu vermeiden. Bis auf zwei offene Fragen am Ende des Fragebogens sind alle Items geschlossen oder halboffen. Halboffene Fragen werden zum einen dann eingesetzt, wenn die Antwort aus Zahlen besteht (Jahre, Monate, Anzahlen von Schüler/innen). Zum anderen ist bei vielen Fragen neben der Auswahl vorgegebener Antworten die Eintragung einer eigenen, sonstigen Antwort möglich, um der explorativen Anlage der Untersuchung Rechnung zu tragen (vgl. Zierer et al., 2013).

Fragen, die auf die Erfassung konkreter Sachverhalten abzielen (z. B. „An welcher Schulform arbeiten Sie als Schulbegleitung?"), erfassen in der Regel Daten auf Nominalskalenniveau bzw. bei der Abfrage von Zahlen auf Absolutskalenniveau. Einschätzungs- und Bewertungsfragen werden in der Regel durch abgestufte Antwortskalen erfasst um einen höheren Genauigkeitsgrad zu erreichen, wobei jede Antwortkategorie bezeichnet wird. Mit Ausnahme der Skalen zur Erfassung von Häufigkeit sind alle Ratingskalen im eingesetzten Fragebogen vierstufig, d. h. auf eine mittlere Antwortkategorie wird verzichtet (vgl. Bühner 2011). Dabei kommen für die Items, die zu Likert-ähnlichen Skalen (Bortz \& Döring 2006; Schnell, Hill \& Esser, 2013) zusammengefasst werden sollen, abgestufte Zustimmungsbezeichnungen zum Einsatz mit den Beschriftungen trifft gar nicht $q u$ - trifft eher nicht - trifft eher zu - trifft voll zu. Für diese Skala wird Intervallskalenniveau angenommen (vgl. Bortz \& Döring, 2006; Bortz \& Schuster, 2010); nach einer Item- und Skalenanalyse ist die Bildung von Skalenmittelwerten vorgesehen. Auch die Ratingskalen, die mit sehr unzufrieden - eher unzufrieden - eher zufrieden - sehr zufrieden, mit sebr unwichtig - eher unwichtig - eher wichtig - sehr wichtig, mit nein eher nein - eherja - ja oder mit abgestuften Häufigkeitsangaben (nie - selten - manchmal-oft - sehr oft) beschriftet sind, werden, wie in der sozialwissenschaftlichen Forschung üblich (z. B. Fussangel, 2008; Quellenberg, 2009), aus forschungspragmatischen Gründen intervallskaliert behandelt. Die Darstellung der Ergebnisse erfolgt bei diesen Skalen nicht nur anhand von Mittelwerten, sondern auch in detaillierter Form anhand der Häufigkeiten der ausgewählten Antwortkategorien. Die in diesen Skalen ggf. zusätzlich vorhandene Antwortkategorie nicht vorhanden wird bei entsprechenden Analysen (wie der Mittelwertberechnung) ausgeschlossen. Zur Erfassung zeitlicher Häufigkeiten kommt zudem eine Ordinalskala zum Einsatz, die mit bislang gar nicht - seltener als monatlich - monatlich - wöchentlich oder fast wöchentlich - täglich oder fast täglich beschriftet ist. Genaue Zeitangaben dieser Art lassen eine objektive Erfassung von Häufigkeiten zu (vgl. Bühner, 2011), jedoch keine intervallskalierte. Aus diesem Grund kommen für unterschiedliche Sachverhalte zwei verschiedene Häufigkeitsskalen zum Einsatz.

Angaben zur Person und zur Maßnahme Schulbegleitung. Der erste Themenkomplex des Fragebogens umfasste (in dieser Reihenfolge) Fragen zur Person und zum Qualifikationshintergrund, Fragen zum Beschäftigungsverhältnis und Fragen zur Maßnahme hinsichtlich der begleiteten Schülerin oder des begleiteten Schülers (wie Schulform, Umfang, Behinderung) sowie zu den ausgeübten Tätigkeiten als Schulbegleitung. Bis auf wenige Ausnahmen beziehen sich die Fragen in diesem Fragebogenteil auf konkrete Sachverhalte (d. h. es gibt objektiv richtige Antworten) und nicht auf Einschätzungen der Befragten. 
Person und Qualifikation: Zu Beginn des Fragebogens wurden die Teilnehmenden um allgemeine Angaben zu ihrer Person gebeten, was eine halboffene Abfrage nach ihrem Alter und geschlossene Fragen zu Geschlecht, höchstem Schulabschluss und formaler Qualifikation/Ausbildung beinhaltete. Letzteres wurde durch zwei Fragen erfasst: Zuerst stand eine Filterfrage danach, ob man eine Ausbildung oder ein Studium abgeschlossen habe oder sich derzeit in Ausbildung oder Studium befinde. In diesem Fall folgte eine detaillierte Auflistung verschiedener Ausbildungs- und Studiengänge, zu denen jeweils angegeben werden konnte, ob man sich zurzeit in ihnen befindet oder sie abgeschlossen hat (Mehrfachantworten möglich). Die Auflistung orientierte sich an Dworschak (2012c) und wurde um weitere Berufe bzw. Studiengänge aus dem Bereich Pädagogik und Pflege ergänzt. Da im Rahmen dieser Studie vor allem interessierte, wie hoch die Fachkraftquote unter den Göttinger Schulbegleiter/innen ist, wurde auf eine Antwortmöglichkeit mit offenem Eintrag verzichtet und stattdessen die Antwortmöglichkeiten „Sonstige Ausbildung [Studium] mit pädagogischem oder pflegerischem Anteil“ und „Sonstige Ausbildung [Studium] ohne pädagogischen oder pflegerischen Anteil“‘. Es folgten weitere, halboffene Abfragen zum Erfahrungs- und Qualifikationshintergrund der Schulbegleiter/innen: Zunächst die Frage, wie lange man bereits als Schulbegleitung tätig ist (in Jahren und Monaten), und darauf die Frage, in welchen Bereichen man zuvor schon Erfahrung hatte. Hierbei wurde in den Antwortmöglichkeiten (Mehrfachauswahl möglich) zwischen ehrenamtlichem und beruflichem Arbeiten mit Kindern und Jugendlichen unterschieden (orientiert an Kauper et al., 2012). Weitere Antwortmöglichkeiten waren „Erziehung eigener Kinder oder Pflegekinder“ und „Zuvor keine Erfahrung mit Kindern oder Jugendlichen“, zusätzlich war ein eigener Eintrag unter „Sonstiges“ möglich. AnschlieBend wurden zwei Fragen zum motivationalen Hintergrund gestellt, und zwar wie lange man schätzungsweise noch als Schulbegleitung tätig sein möchte (in Jahren und Monaten) und aus welchen Gründen man Schulbegleiter/in geworden ist. Für die zweite Frage wurden in Orientierung an Pohlmann und Möller (2010) sieben mögliche Gründe und „Sonstige Gründe“ mit Eintragmöglichkeit vorgegeben, zu denen die Befragten auf einer vierstufigen Likert-Skala ihre Zustimmung oder Ablehnung ausdrücken sollten. Neben Gründen, die pädagogisches Interesse bzw. Interesse an den Arbeitsinhalten (z. B. „,Weil ich gerne mit Kindern/Jugendlichen arbeite") und generelle Nützlichkeitserwägungen (z. B. „Weil die Arbeitszeiten günstig für mich sind“") widerspiegeln (siehe Pohlmann \& Möller, 2010), wurden Items hinzugefügt, die der Besonderheit Rechnung tragen, dass für Schulbegleitung keine formale Qualifikation vorausgesetzt wird, nämlich „Weil ich in meinem Wunsch-Tätigkeitsfeld keine Arbeit gefunden habe“, „Weil ich für Schulbegleitung keine formale Qualifikation brauche" und „Weil ich mich beruflich neu orientieren möchte“.

Beschäftigungsverbältnis: Hinsichtlich der aktuellen Maßnahme, innerhalb derer die befragten Schulbegleiter/innen tätig sind, wurde zunächst nach dem Kostenträger und nach dem Arbeitgeber gefragt. Als Antwortmöglichkeiten standen im ersten Fall eines der vier Ämter bzw. „Weiß ich nicht“" und im zweiten Fall „Mit einem freien Träger (z. B. Haus mit vielen Etagen, JSN, Lebenshilfe)“, „Mit dem Jugend- oder Sozialamt“ und „Mit den Eltern des Schülers/der Schülerin“" zur Auswahl. In beiden Fällen konnte alternativ ein eigener Eintrag vorgenommen werden. Weiterhin wurde gefragt, ob die Tätigkeit als Schulbegleitung auf Honorarbasis, im Angestelltenverhältnis oder im Rahmen eines Freiwilligendienstes oder Ehrenamts ${ }^{32}$ erfolgte. Es wurden außerdem die Gesamtlaufzeit des aktuellen Vertrags als Schulbegleitung erfragt und die eigene Zufriedenheit mit der Entlohnung, der Laufzeit des Vertrags, den Urlaubsregelungen und den Regelungen im Krankheitsfall. Auf einer vierstufigen Skala sollte die eigene Zufriedenheit mit diesen Aspekten, die sich bei der Literaturrecherche und den Vorgesprächen als zentrale Kritikpunkte im Beschäftigungsverhältnis herausgestellt hatten, angegeben werden. Schließlich wurde noch ob um die Angabe gebeten, ob die einstellende Einrichtung/der Vertragspartner Schulungen anbietet und ob man bereits an einer oder mehr Schulungen teilgenommen hat.

Begleitete Scbüler/innen: Hinsichtlich des begleiteten Schülers interessierte vorab, ob man aktuell für mehr als eine/n Schüler/in tätig sei; in diesem Fall wurde darum gebeten, alle folgenden Fragen auf den Schüler oder die Schülerin zu beziehen, für den oder die man hauptsächlich tätig ist. Auf diese Weise wird sichergestellt, dass gleichmäßig die Maßnahmen mit dem höchsten Umfang diejenigen sind, auf die sich die Angaben der Schulbegleiter/innen beziehen. Zum begleiteten Kind oder Jugendlichen wurde daraufhin mit geschlossenen Fragen die Behinderungsart (seelisch, geistig oder körperlich) und die Schulform erfasst. Außerdem sollte der Stundenumfang der Begleitung eingetragen werden. Zuletzt wurde um die Einschätzung gebeten,

32 Zwar hatten sich in den Vorgesprächen zum Fragebogen keine Hinweise darauf ergeben, dass in Göttingen Schulbegleiter/innen ehrenamtlich tätig sind, in der Fachliteratur wird dieser Fall aber erwähnt. 
wie häufig bestimmte Unterrichtsformen („Lehrerzentrierter Frontalunterricht“”, „Gruppenarbeit“, „Partnerarbeit", „Einzelarbeit") in den begleiteten Schulstunden vorkommen (nie - selten - manchmal - oft - sebr $o f t)$.

Tätigkeitsprofil: Die Befragten sollten einschätzen, wie häufig sie bestimmte Tätigkeiten als Schulbegleitung ausführen (nie - selten - manchmal - oft - sehr oft). Die Liste der Tätigkeiten orientierte sich an Dworschak (2012c) und Henn et al. (2014) sowie an selbst geführten Vorgesprächen zur Studie. Gemäß der in Kapitel 2 vorgenommenen Einteilung der schülerbezogenen Tätigkeiten in Unterstützung bei lebenspraktischen Anforderungen, Unterstützung bei der Emotions- und Verhaltenskontrolle sowie didaktische Unterstützung wurden zu jedem Bereich Aufgaben mit konkreten Beispieltätigkeiten (z. B. „Pflegerische Tätigkeiten und Mobilität (z.B. Rollstuhl schieben, bei Toilettengang unterstützen)") formuliert, von denen aufgrund der Vorrecherche angenommen werden kann, dass sie von Schulbegleiter/innen ausgeführt werden. Für eine detaillierte Auflistung siehe Tabelle 1. Der Bereich nicht schülerbezogener, sondern mittelbarer Aufgaben fand hier keine Berücksichtigung, da er im Wesentlichen durch die Abfrage im Themenkomplex Einbindung und Kooperation abgedeckt wurde. Gemäß der explorativen Anlage des Fragebogens wurden aber drei Freifelder für eigene Eintragungen von Tätigkeiten zur Verfügung gestellt.

Tab. 1: Zuordnung der Fragebogenitems zum Tätigkeitsprofil zu verschiedenen Aufgabenbereichen

\begin{tabular}{ll}
\hline Aufgabenbereich & Tätigkeit \\
\hline $\begin{array}{l}\text { Pflegerische Tätigkeiten und Mobilität (z. B. Rollstuhl schieben, bei Toilettengang } \\
\text { unterstützen) } \\
\text { lebenspraktischen } \\
\text { Anforderungen }\end{array}$ & Unterstützung bei der Verwendung von Hilfsmitteln (z. B. PC) \\
& Orientierung geben (z. B. vom Bus abholen, Wechsel in andere Räume begleiten) \\
& Beaufsichtigung Ihres Schülers/Ihrer Schülerin während der Pausea \\
\hline Unterstützung bei der & Unterstützung bei der emotionalen Regulierung (z. B. beruhigen, ermuntern) \\
Emotions- und & Unterstützung von Sozialkontakten mit anderen Schüler/innen \\
Verhaltenskontrolle & Intervention bei aggressivem oder autoaggressivem Verhalten \\
& Intervention bei Regelverstößen (z. B. Regelverstoß ansprechen) \\
\hline Unterstützung in Einzelarbeitsphasen (z. B. Aufgaben erklären, bei der Lösungs- \\
suche helfen) \\
Unterstützung in Gruppenarbeitsphasen (z. B. Aufgaben erklären, bei der Lö- \\
sungssuche helfen) \\
Unterstützung im Frontalunterricht (z. B. Wiederholung oder Erklärung dessen, \\
was die Lehrkraft sagt) \\
Unterrichtsbezogene Unterstützung von anderen Schüler/innen \\
Unterstützung
\end{tabular}

Anmerkungen. Im Fragebogen selbst sind nur die Tätigkeiten, nicht die Tätigkeitsbereiche aufgeführt.

a Diese Aufgabe wird im Ergebnisteil der Unterstützung der Emotions- und Verhaltenskontrolle zugeordnet.

Die letzten Fragen in diesem ersten Themenkomplex bezogen sich auf die Anzahl von Schulbegleiter/innen in der Klasse des begleiteten Kindes oder Jugendlichen sowie der Ja/Nein-Frage nach der Existenz einer Koordinationskraft für alle Schulbegleitungen an der Einsatzschule.

Fragen zu Einbindung und Kooperation. Der zweite Themenkomplex des Fragebogens umfasste (in dieser Reihenfolge) Fragen zur berufsbezogenen Selbstwirksamkeitserwartung, zur Häufigkeit von Austausch mit verschiedenen Personengruppen, zur Einbindung in die Schulgemeinschaft, zur Kooperation mit 
der Klassenleitung, zu möglichen Wirkungen der Kooperation mit der Klassenleitung (emotionale Entlastung, wahrgenommene Wertschätzung, Rollensicherheit, Arbeitszufriedenheit) und zur Bewertung des Austauschs mit verschiedenen Personengruppen (Wichtigkeit und Zufriedenheit). Zuletzt standen die Einschätzung der Gesamtzufriedenheit mit der Einbindung und Zusammenarbeit in der Schule sowie die Frage nach Gründen für diese Bewertung, bevor offene Angaben mit eigenen Anmerkungen gemacht werden konnten. Die Items zu berufsbezogener Selbstwirksamkeitserwartung sowie zur Kooperationspraxis mit der Klassenleitung und möglichen Kooperationswirkungen werden einer detaillierten Item- und Skalenanalyse unterzogen. Mit Ausnahme der Angabe der Häufigkeit von Austausch mit verschiedenen Personengruppen beziehen sich alle Fragen in diesem Fragebogenteil auf Einschätzungen und Bewertungen der Befragten. Die Items zur Kooperationspraxis mit der Klassenleitung (und ein Teil der Items zur Einbindung in die Schulgemeinschaft) zielen dabei auf die Einschätzung (des Zutreffens) prinzipiell beobachtbarer Verhaltensweisen ab, alle anderen Items auf internale und prinzipiell nicht beobachtbare Umstände (Bühner, 2011). Die Fragen, die sich explizit auf eine Bewertung der Zusammenarbeit beziehen, standen am Ende des Fragebogens (vgl. Zierer et al., 2013). Für die nun folgende nähere Beschreibung werden die Fragen abweichend von der Reihenfolge im Fragebogen inhaltlich gruppiert; in dieser Form werden sie auch im Ergebnisteil wieder aufgegriffen.

Häufigkeit und Bewertung von Austauscb: Um einschätzen zu können, welche Personen als Kooperationspartner/innen im Rahmen von Schulbegleitung eine Rolle - und welche - spielen, wurden die Befragten gebeten, die Häufigkeit des Austauschs mit verschiedenen Personengruppen, die Wichtigkeit hiervon und die Zufriedenheit hiermit anzugeben. Ziel dieser dreigliedrigen Abfrage ist, die Kooperationsbeziehungen von Schulbegleiter/innen, deren Tätigkeit an einem Knotenpunkt zwischen verschiedenen Personengruppen angesiedelt ist (Knuf, 2013; siehe Kapitel 2), nicht nur hinsichtlich der zeitlichen Frequenz zu beleuchten, sondern auch ihre subjektive Bewertung zu erfassen. Die Bezeichnung Austausch wurde bewusst gewählt um jegliche Formen von arbeitsbezogenem Kontakt einzuschließen. Als Personen(gruppen) waren aufgeführt: die Schulleitung, die Klassenleitung, die zuständige sonderpädagogische Lehrkraft, weiteres an der Schule tätiges Personal, andere an der Schule tätige Schulbegleiter/innen, die Koordinationskraft für Schulbegleiter/innen an der Schule, die Leitung des Ganztags, außerschulische Kooperationspartner, die arbeitgebende Einrichtung sowie die Eltern des Schülers oder der Schülerin. Zusätzlich wurde eine Eintragmöglichkeit für sonstige Personen bereitgehalten. Zu den drei Aspekten (Häufigkeit, Wichtigkeit, Zufriedenheit) konnte auf mehrstufigen Skalen jeweils zu jeder Person(engruppe) die entsprechende Antwort ausgewählt werden, zusätzlich war jeweils die Antwortmöglichkeit nicht vorbanden gegeben. Für die Häufigkeit des Austauschs konnte zwischen bislang gar nicht - seltener als monatlich - monatlich - wöchentlich oder fast wöchentlich täglich oder fast täglich ausgewählt werden, für die Wichtigkeit des Austauschs zwischen sebr unwichtig - eher unwichtig - eher wichtig - sebr wichtig und für die Zufriedenheit mit dem Austausch zwischen sebr unzufrieden eher unzufrieden - eher zufrieden - sehr zufrieden.

Selbstwirksamkeit, Kooperationspraxis und Kooperationswirkungen: Zur Erfassung von berufsbezogener Selbstwirksamkeitserwartung sowie der Kooperationspraxis mit der Klassenleitung und möglichen Kooperationswirkungen wurden Itembatterien entwickelt; aus den jeweiligen Items sollten Skalenmittelwerte gebildet werden. Dabei wird wie folgt vorgegangen: Da unabhängig von der Verteilung der erhobenen Daten ab einer Stichprobengröße von $N=30$ davon ausgegangen werden kann, dass die Mittelwertverteilung einer Normalverteilung entspricht (Bortz \& Schuster, 2010), wird auf eine Prüfung der Werteverteilung verzichtet. Orientiert am Vorgehen zur Erstellung einer Likert-Skala (Bortz \& Döring 2006; Bühner, 2011; Kuckartz, Rädiker, Ebert \& Schehl, 2013; Schnell et al., 2013) erfolgt die Skalenanalyse anhand der Trennschärfen der einzelnen Items (den Werten der korrigierten Item-Skala-Korrelation) und der Reliabilität der Skala (der internen Konsistenz). Als Kennwert für die Reliabilität der Skala wird Cronbachs Alpha für standardisierte Items verwendet (Bortz \& Döring 2006) ${ }^{33}$. Prinzipiell sollten Items, die eine Trennschärfe unter .4 aufweisen, ausgeschlossen werden, wenn dem Ausschluss keine inhaltlichen Erwägungen entgegenstehen und wenn sich die Reliabilität der Skala durch den Ausschluss erhöht ${ }^{34}$ (vgl. Bühner, 2011; Kuckartz et al., 2013; Schnell et al., 2013). Dabei ist zu beachten, dass Cronbachs Alpha auch von der Anzahl der Items abhängt (Bortz \& Döring, 2006). Der Ausschluss von Items erfolgt deshalb Schritt für Schritt, d. h. nach Ausschluss des

33 Zuvor erfolgte die Prüfung auf ausschließlich positive Interitemkorrelationen (Bortz \& Döring, 2006).

34 Vor dem Ausschluss von Items ist zu generell zu bedenken, dass hierdurch ggf. die Breite des Konstrukts geschmälert wird; umfasst das zu messende Merkmal verschiedene Facetten, die sich in heterogenen Items abbilden, kann ein Ausschluss von Items aufgrund statistischer Kennwerte zwar die Reliabilität/interne Konsistenz der Skala erhöhen, allerdings zu Lasten der Inhaltsvalidität. Dies wird bei der Itemselektion berücksichtigt. 
Items mit der geringsten Trennschärfe erfolgt eine erneute Analyse für alle verbliebenen Items, bevor auf dieser Basis ggf. ein weiteres Item ausgeschlossen wird (Kuckartz et al., 2013). Ein Wert von $a>.7$ wird bei noch nicht erprobten Skalen als zufriedenstellend angesehen (vgl. Bortz \& Döring, 2006; Schnell et al., 2013; Zierer et al., 2013). Mit fehlenden Werten wird wie folgt umgegangen: Aufgrund der geringen Itemzahl pro zu bildender Skala werden Fälle, in denen mindestens ein Wert fehlt, sowohl bei der Itemanalyse zur Skalenbildung als auch bei Bildung der letztendlichen Skalenmittelwerte ausgeschlossen. Im Folgenden werden nun die einzelnen Skalen beschrieben.

Die genaue Formulierung der Items sowie sämtliche Item- und Skalenkennwerte werden in Tabelle 2 aufgeführt. An dieser Stelle wird deshalb nur in kurzer Form auf die Entwicklung der Items und die Skalenbildung eingegangen. Für die Erfassung der berufsbezogenen Selbstwirksamkeitserwartung konnte auf die Lebrer-Selbstwirksamkeitsskala von Schmitz und Schwarzer (2000) zurückgegriffen werden. Gegenüber der Skala Allgemeine Selbstwirksamkeitserwartung (Schwarzer \& Jerusalem, 1999) hat die berufsbezogene Skala den Vorteil, berufsbezogen spezifischere Ergebnisse zu liefern (Schmitz \& Schwarzer, 2000). Die Lehrer-Selbstwirksamkeitsskala deckt die Bereiche ab, die nach Rudow (1995) zentral für den Lehrerberuf sind: berufliche Leistung, berufliche Weiterentwicklung, soziale Interaktionen mit Schüler/innen, Eltern und Kolleg/innen sowie Umgang mit Berufsstress. Diese Bereiche decken auch die Anforderungsbereiche von Schulbegleiter/innen ab, wobei die Interaktion mit den einzelnen, begleiteten Schüler/innen im Mittelpunkt steht. Dieser Umstand wurde bei der Adaption der Skala und der Umformulierung der Items beachtet. Insgesamt konnten von den 10 Items der Originalskala sechs sinnvoll auf Schulbegleitung angepasst werden ${ }^{35}$; hiervon wurden fünf in die endgültige Skala übernommen. Ausschlaggebend für die Entscheidung, auch ein Item mit einer Trennschärfe von .35 einzuschließen, war, dass die Originalskala Lehrer-Selbstwirksamkeitserwartung nicht dem Ziel einer hohen internen Konsistenz, sondern mit dem Ziel, alle Facetten eines heterogenen Konstrukts abzubilden, entwickelt wurde (Schmitz \& Schwarzer, 2000). ${ }^{36}$

Bei der Itemformulierung zur Kooperationspraxis und zu Kooperationswirkungen wird durchgängig die Bezeichnung Klassenleitung verwendet, da diese Bezeichnung im Gegensatz zu Klassenlehrkraft auch Klassenlehrerteams einschließt. Bei der Zusammenarbeit von Klassenleitung und Schulbegleitung ist zu bedenken, dass der Klassenleitung durch ihre Verantwortlichkeit für die gesamte Klasse einschließlich der begleiteten Schüler/innen eine prinzipiell höhergestellte Position zukommt (siehe Kapitel 4). Diesem Hierarchieverhältnis wird bei der Operationalisierung der Kooperationspraxis und Kooperationswirkungen Rechnung getragen, indem ein großer Teil der Items darauf abzielt zu erfassen, inwieweit die Klassenleitung kooperative Verhaltensweisen gegenüber der Schulbegleitung zeigt. Sprachlich kommt dies z. B. darin zum Ausdruck, dass in vielen Items die Klassenleitung das Subjekt und die Versuchsperson das Objekt des Satzes bildet.

Zur Kooperationspraxis waren zwei Skalen vorgesehen, Koordination und Kokonstruktion. Insgesamt wurden 11 Items neu entwickelt, die Verhaltensweisen beschreiben, von denen aufgrund der Vorrecherche angenommen werden kann, dass sich mit ihnen die zentralen kooperativen Verhaltensweisen von Klassenleitungen und Schulbegleiter/innen beschreiben lassen. Zum Teil war dabei eine Orientierung an den Skalen Austausch bzw. Kokonstruktion von Fussangel (2008), der Skala Schülerberogene interprofessionelle Kooperation (Dizinger \& Böhm-Kasper, 2012) sowie der Checkliste für Teamarbeit im inklusiven Unterricht von Arndt et al. (2014) möglich. Vier Items beziehen sich auf Verhaltensweisen, die sich im Sinne einer weniger anspruchsvollen Kooperationsform als Koordination bezeichnen lassen und sieben Items auf Verhaltensweisen, die sich im Sinne einer anspruchsvolleren Kooperationsform als Kokonstruktion beschreiben lassen (siehe Kapitel 4). Es zeigt sich, dass beide Skalen hoch miteinander korrelieren $(r=.84, p<.001)$. Eine faktorenanalytische Überprüfung der Dimensionalität der Skalen (siehe Bortz \& Döring, 2006) kann aufgrund der geringen Stichprobengröße nicht vorgenommen werden, weshalb zusätzlich eine Prüfung der statistischen Kennwerte einer Gesamtskala für Kooperation erfolgte. Anhand der berücksichtigten Kennwerte (InterItem-Korrelationen, Item-Skala-Korrelationen, mittlere Inter-Item-Korrelationen, Cronbachs Alpha) lässt sich nicht beurteilen, ob die zwei Kooperationsskalen tatsächlich zwei empirisch trennbare Dimensionen von Kooperation erfassen. Alle Zusammenhangsberechnungen im Rahmen der Forschungsfragen bzw. der

35 Das Antwortformat einer vierstufigen Likert-Skala wurde übernommen; zur Wahrung der Einheitlichkeit innerhalb des Fragebogens wurden die Antwortbeschriftungen angepasst.

36 Gerechtfertigt ist diese Entscheidung auch durch die akzeptablen Korrelationswerte dieses Items mit den anderen Items der Skala $(r$ jeweils > .2) und dadurch, dass bei Ausschluss dieses Items weitere Items unter die Trennschärfegrenze von .4 fallen. 
Hypothesenprüfung werden deshalb einmal für die Skala Koordination und für die Skala Kokonstruktion durchgeführt und einmal für eine Gesamtskala Kooperationspraxis, die die Items beider Skalen zusammenfasst. Hinsichtlich der Wirkungen von Kooperation mit der Klassenleitung auf die Schulbegleiter/innen wurden vier Itembatterien mit jeweils vier Items gebildet. Neben Items, die die emotionale Entlastung durch Kooperation erfassen sollen, wurden Itembatterien zur Erhebung der wahrgenommenen Wertschätzung, zur Rollensicherheit und zur Arbeitszufriedenheit entworfen. Die Items zur Erfassung der Konstrukte Emotionale Entlastung und Wahrgenommene Wertschätzung bezogen sich auch sprachlich explizit auf die Klassenleitung bzw. die Zusammenarbeit mit der Klassenleitung. Für die beiden anderen Konstrukte, Rollensicherheit und Arbeitszufriedenheit, war nur in der Instruktion festgehalten, dass sie sich auf die Zusammenarbeit mit der Klassenleitung beziehen. Bei der Entwicklung der Items zu emotionaler Entlastung konnten teilweise Items der gleichnamigen Skala von Fussangel (2008) adaptiert werden. Orientierung bot auch die Skala Entlastung durch interprofessionelle Kooperation von Dizinger und Böhm-Kasper (2012). Die Items zu Wertschätzung wurden teilweise mit Rückgriff auf die Teamcheckliste von Arndt et al. (2014) entwickelt, die Items zu Rollensicherheit allein anhand der Konzeptspezifikation (siehe Kapitel 4). Die Skala Arbeitszufriedenheit wurde in leicht adaptierter Form fast vollständig von Mullis, Martin, Kennedy und Foy (2007) bzw. Bos et al. (2010) übernommen, die im Rahmen von PIRLS/IGLU die Berufszufriedenheit von Lehrkräften erhoben haben. Ergänzt wurde das Item „Meine Arbeit als Schulbegleitung frustriert mich“.

Die Skalenanalysen ergaben überwiegend zufriedenstellende Werte, lediglich die Skalen Rollensicherheit und Arbeitszufriedenheit bedurften einer genaueren Analyse. Ebenso wie bei der Skala Berufsbezogene Selbstwirksamkeit wurde bei Bildung der Skala Rollensicherheit ein Item mit einer Trennschärfe von .35 zugunsten der Erfassung der inhaltlichen Breite des Konstrukts eingeschlossen. Legitimiert wird diese Entscheidung auch durch die akzeptablen Korrelationswerte dieses Items mit den anderen Einzelitems $(r=.19$, .31, .4). Bei der Skala Arbeitszufriedenheit wurde ein Item ausgeschlossen, dass mit zwei der drei anderen Items nahezu nullkorreliert. Inhaltlich lässt sich das Item von den anderen insofern trennen, als dass es als einziges auf eine Bewertung abzielt, die nicht explizit nach den eigenen positiven Emotionen fragt. ${ }^{37}$

37 Auch bei IGLU wurde dieses Item von der letztlich gebildeten Skala ausgeschlossen. 
Tab. 2: Itemkennwerte und Skalenkennwerte zur Selbstwirksamkeitserwartung und zur Kooperationspraxis

\begin{tabular}{|c|c|c|}
\hline Item & $M(S D)$ & $r_{i t}$ \\
\hline \multicolumn{3}{|l|}{ Skala Berufsbezogene Selbstwirksamkeitserwartung $(a=.7, n=44, M=3.49, S D=.4)$} \\
\hline $\begin{array}{l}\text { Ich kann auch in problematischen Situationen mit meinem Schüler/meiner Schüle- } \\
\text { rin in guten Kontakt kommen, wenn ich mich darum bemühe }\end{array}$ & $3.61(.54)$ & .58 \\
\hline $\begin{array}{l}\text { Ich bin mir sicher, dass ich mich in Zukunft auf Probleme meines Schülers/meiner } \\
\text { Schülerin noch besser einstellen kann }\end{array}$ & $3.30(.70)$ & .35 \\
\hline $\begin{array}{l}\text { Selbst wenn etwas nicht so läuft wie geplant, bin ich mir sicher, die notwendige Ge- } \\
\text { lassenheit bewahren zu können }\end{array}$ & $3.61(.50)$ & .60 \\
\hline $\begin{array}{l}\text { Selbst wenn es mir mal nicht so gut geht, kann ich doch als Schulbegleiter/in immer } \\
\text { noch gut auf meine/n Schüler/in eingehen }\end{array}$ & $3.32(.56)$ & .46 \\
\hline $\begin{array}{l}\text { Auch wenn ich mich noch so sehr für die Entwicklung meines Schülers/meiner } \\
\text { Schülerin engagiere, kann ich nicht viel ausrichten }{ }^{a}\end{array}$ & $3.32(.71)$ & .27 \\
\hline $\begin{array}{l}\text { Ich kann kreative Ideen entwickeln, mit denen ich ungünstige Verhaltensmuster } \\
\text { meines Schülers/meiner Schülerin verändere }\end{array}$ & $3.30(.63)$ & .40 \\
\hline \multicolumn{3}{|l|}{ Skala Koordination $(a=.83, n=44, M=3.52, S D=.59)^{\mathrm{b}}$} \\
\hline $\begin{array}{l}\text { Die Klassenleitung und ich haben klare Absprachen getroffen, wie ich mich in be- } \\
\text { stimmten Situationen verhalten soll }\end{array}$ & $3.20(.93)$ & $.62[.62]^{\mathrm{b}}$ \\
\hline Ich tausche mich mit der Klassenleitung über meine/n Schüler/in aus & $3.42(.80)$ & $.76[.82]$ \\
\hline Wichtige Informationen werden von der Klassenleitung an mich weitergegeben & $3.80(.41)$ & $.64[.78]$ \\
\hline $\begin{array}{l}\text { Ich habe jederzeit die Möglichkeit, von der Klassenleitung Informationen über mei- } \\
\text { nen Schüler/meine Schülerin einzuholen }\end{array}$ & $3.57(.66)$ & $.80[.76]$ \\
\hline \multicolumn{3}{|l|}{ Skala Kokonstruktion $(a=.93, n=43, M=3.4, S D=.66)^{\mathrm{b}}$} \\
\hline Ich erhalte von der Klassenleitung Feedback zu meiner Arbeit & $3.26(.93)$ & $.72[.76]^{\mathrm{b}}$ \\
\hline $\begin{array}{l}\text { Ich werde von der Klassenleitung nach meiner Einschätzung meines Schülers/mei- } \\
\text { ner Schülerin gefragt }\end{array}$ & $3.53(.77)$ & $.88[.87]$ \\
\hline $\begin{array}{l}\text { Die Klassenleitung und ich überlegen gemeinsam, was in bestimmten Situationen zu } \\
\text { tun ist }\end{array}$ & $3.44(.85)$ & $.89[.90]$ \\
\hline $\begin{array}{l}\text { Ich gebe Rückmeldung an die Klassenleitung, wenn ich den Eindruck habe, dass } \\
\text { mein/e Schüler/in im Unterricht unter- oder überfordert ist }\end{array}$ & $3.80(.47)$ & $.67[.68]$ \\
\hline $\begin{array}{l}\text { Arbeitsbezogene Probleme und Fragen klären die Klassenleitung und ich gemein- } \\
\text { sam }\end{array}$ & $3.37(.82)$ & $.84[.84]$ \\
\hline $\begin{array}{l}\text { Die Klassenleitung und ich entwickeln im Gespräch einen gemeinsamen Blick auf } \\
\text { die Entwicklung meines Schülers/meiner Schülerin }\end{array}$ & $3.44(.80)$ & $.84[.85]$ \\
\hline $\begin{array}{l}\text { Die Klassenleitung fragt (auch) bezüglich Themen, die nicht direkt meine/n Schü- } \\
\text { ler/in betreffen, nach meiner Einschätzung (z. B. bezüglich anderer Schüler/innen } \\
\text { oder der Unterrichtsgestaltung) }\end{array}$ & $2.88(.96)$ & $.63[.62]$ \\
\hline
\end{tabular}

Anmerkung. Angegeben sind Mittelwerte $(M)$, Standardabweichung $(S D)$ und Trennschärfe $\left(r_{i t}\right)$ der Items sowie die Skalenkennwerte Cronbachs Alpha für standardisierte Items (a) und die jeweilige Stichprobengröße ( $n$ ). Vierstufiges Antwortformat (trifft gar nicht $z_{u}$ - trifft eher nicht - trifft eher $₹ u$ - trifft voll $\left.z u\right)$. Das grau hinterlegte Item wird ausgeschlossen. Die Skalenkennwerte und die Trennschärfen der anderen Items beziehen sich auf die Skala nach dem Ausschluss des grau hinterlegten Items.

a Negativ formuliertes Item; angegeben ist der Mittelwert nach der Umpolung.

b Die Skalen Koordination und Kokonstruktion werden in den Analysen zusammenfasst zur Skala Kooperationspraxis $(M=3.42$, $S D=.63, a=.95, n=42)$. In den eckigen Klammern sind die Trennschärfen der Items in dieser Skala angegeben. 
Tab. 3: Itemkennwerte und Skalenkennwerte zum arbeitsbezogenen Erleben

\begin{tabular}{|c|c|c|}
\hline Item & $M(S D)$ & $r_{i t}$ \\
\hline \multicolumn{3}{|l|}{ Skala Emotionale Entlastung $(a=.94, n=44, M=3.44, S D=.72)$} \\
\hline $\begin{array}{l}\text { Durch die Zusammenarbeit mit der Klassenleitung fühle ich mich bei Problemen } \\
\text { mit meinem Schüler/meiner Schülerin nicht auf mich allein gestellt }\end{array}$ & $3.34(.86)$ & .74 \\
\hline $\begin{array}{l}\text { Ich kann mich bei der Klassenleitung absichern, wie ich mit arbeitsbezogenen Prob- } \\
\text { lemen umgehe }\end{array}$ & $3.48(.73)$ & .90 \\
\hline Bei Problemen finde ich bei der Klassenleitung ein offenes Ohr & $3.57(.80)$ & .89 \\
\hline $\begin{array}{l}\text { Ich kann mit der Klassenleitung offene Gespräche über arbeitsbezogene Probleme } \\
\text { führen, die mich entlasten }\end{array}$ & $3.39(.78)$ & .87 \\
\hline \multicolumn{3}{|l|}{ Skala Wertschätəung ( $a=.91, n=45, M=3.54, S D=.63)$} \\
\hline Ich erfahre bei meiner Arbeit Wertschätzung durch die Klassenleitung & $3.6(.75)$ & .83 \\
\hline $\begin{array}{l}\text { Meine Meinung zu arbeitsbezogenen Problemen wird von der Klassenleitung res- } \\
\text { pektiert }\end{array}$ & $3.47(.76)$ & .82 \\
\hline $\begin{array}{l}\text { Meine Einschätzung meines Schülers/meiner Schülerin hat für die Klassenleitung } \\
\text { Gewicht }\end{array}$ & $3.67(.52)$ & .76 \\
\hline Ich habe das Gefühl, dass ich als Teil eines professionellen Teams angesehen werde & $3.42(.78)$ & .81 \\
\hline \multicolumn{3}{|l|}{ Skala Rollensicherbeit ( $a=.73, n=45, M=3.37, S D=.54$ ) } \\
\hline Ich bin mir in meiner Rolle als Schulbegleitung oft unsicher ${ }^{b}$ & $3.62(.58)$ & .62 \\
\hline $\begin{array}{l}\text { Die unterschiedlichen Erwartungen, die an mich als Schulbegleitung herangetragen } \\
\text { werden, verunsichern mich }{ }^{\mathrm{b}}\end{array}$ & $3.51(.87)$ & .45 \\
\hline Mein Aufgabenbereich als Schulbegleitung ist klar abgesteckt & $2.84(.93)$ & .35 \\
\hline Ich weiß, was von mir als Schulbegleitung erwartet wird & $3.51(.59)$ & .58 \\
\hline \multicolumn{3}{|l|}{ Skala Arbeitszufriedenheit ( $a=.83, n=45, M=3.49, S D=.64$ ) } \\
\hline Ich bin gerne Schulbegleiter/in & $3.67(.48)$ & .67 \\
\hline Ich hatte zu Beginn meiner Tätigkeit als Schulbegleitung mehr Begeisterung als jetzta & $3.22(.10)$ & .68 \\
\hline Als Schulbegleiter/in mache ich eine wichtige Arbeit & $3.73(.48)$ & .02 \\
\hline Meine Arbeit als Schulbegleitung frustriert micha & $3.58(.72)$ & .70 \\
\hline
\end{tabular}

Anmerkung. Angegeben sind Mittelwerte $(M)$, Standardabweichung $(S D)$ und Trennschärfe $\left(r_{i t}\right)$ der Items sowie die Skalenkennwerte Cronbachs Alpha für standardisierte Items (a) und die jeweilige Stichprobengröße (n). Vierstufiges Antwortformat (trifft gar nicht $z u$ - trifft eher nicht - trifft eher $₹ u$ - trifft voll $₹ u$ ). Das grau hinterlegte Item wird ausgeschlossen. Die Skalenkennwerte und die Trennschärfen der anderen Items beziehen sich auf die Skala nach dem Ausschluss des grau hinterlegten Items.

a Negativ formuliertes Item; angegeben ist der Mittelwert nach der Umpolung.

Einbindung und Zusammenarbeit mit der Schule: Zur Messung, inwieweit sich Schulbegleiter/innen allgemein in ihre Schule eingebunden fühlen, wurden zwei Items der Skala Einbindung in die Schulgemeinschaft des StEGFragebogens (Quellenberg, 2009) mit geringfügigen Änderungen übernommen („Ich empfinde mich als Teil der Schulgemeinschaft" und „Ich werde bei offiziellen schulischen Veranstaltungen (z. B. Festen, Tag der offenen Tür) eingeladen“. Zusätzlich wurden zwei schulbegleiterspezifische Items eingefügt, nämlich „Ich werde zu Klassenkonferenzen, die meine/e Schüler/in betreffen, eingeladen“ und „Ich werde zu Einzelfallbesprechungen/Hilfeplangesprächen/Förderplangesprächen bzgl. meines Schülers/meiner Schülerin eingeladen“. Alle vier Items konnten auf einer vierstufigen Likert-Skala mit trifft gar nicht $2 u$ - trifft eher nichttrifft eher $₹ u$ - trifft $z u$ beantwortet werden. Die Frage nach der Gesamtzufriedenheit mit der Zusammenarbeit und Einbindung an der Schule war als Filterfrage angelegt und konnte mit nein - eher nein - eher ja - ja beantwortet werden. Bei Auswahl von nein oder eher nein erfolgte die Weiterleitung zur Frage nach den drei wichtigsten Gründen für die Unzufriedenheit, bei Auswahl von ja oder eher ja entsprechend die Frage nach 
den drei wichtigsten Gründen für die Zufriedenheit (Mehrfachantworten möglich). Es wurden jeweils soweit möglich, analog formulierte - Gründe vorgegeben (z. B. mangelnde Anleitung meiner Tätigkeit bzw. genaue Anleitung meiner Tätigkeit) sowie eine offene Eintragmöglichkeit für sonstige Gründe. Um den Teilnehmenden die Möglichkeiten zu geben, eigene Anliegen einzubringen, wurden am Ende des Fragebogens zwei offene Fragen gestellt. Mit einer wurde nach Verbesserungsvorschlägen, die die Zusammenarbeit und Einbindung in der Schule betreffen, gefragt, mit der anderen um Anmerkungen zu der Umfrage oder der Ergänzung wichtiger Fragen, die nicht vorkamen, gebeten.

\subsection{Vorgehen bei der Datenanalyse}

Eine Fehlersichtung anhand von Häufigkeitsanalysen (etwa auf Eingabefehler der Befragten beim Eintrag von Zahlenantworten) ließ keine Auffälligkeiten erkennen. Eine manuelle Datenkorrektur wurde lediglich an einer Stelle vorgenommen. ${ }^{38}$ Die Prüfung fehlender Werte ergab keine systematischen Häufungen bei bestimmten Items oder bestimmten Versuchspersonen (ausgenommen dem Umstand, dass in zwei Fällen ein Abbruch der Bearbeitung gegen Ende des Fragebogens erfolgte); es wurden keine Items und keine Fälle ausgeschlossen. Für die Analyse einzelner Items ergeben sich damit teilweise unterschiedliche Fallzahlen. Aufgrund nur geringer Abweichungen (zwischen 43 und 47, in wenigen Fällen auch 41 und 42; 38 in einem Fall, auf den gesondert eingegangen wird) wird auf eine gesonderte Angabe der Fallzahlen je Item im Ergebnisbericht weitgehend verzichtet. Prozent- und andere Anteilszahlen beziehen sich der besseren Vergleichbarkeit halber stets nur auf die gültigen Fälle, d. h. Befragte mit fehlenden Werten bei dem entsprechenden Item sind darin nicht eingeschlossen.

Entsprechend den in Kapitel 4 formulierten Forschungsfragen werden die erhobenen Daten deskriptiv und inferenzstatistisch ausgewertet. Aufgrund der geringen Stichprobengröße von $N=47$ werden neben Verfahren der Deskriptivstatistik nur bivariate Testverfahren eingesetzt. Die Bewertung des Signifikanzniveaus der Testergebnisse entspricht den gängigen Konventionen. Bei $p \leq .05$ wird das Ergebnis als signifikant gewertet, bei $p \leq .01$ als hochsignifikant und $p \leq .001$ als höchstsignifikant. Berichtet werden auch $p-$ Werte, die über .05, aber unter oder bei 0.1 liegen und auf Tendenzen hinweisen. Da der Stichprobenumfang höher als $n=30$ liegt, können für alle Berechnungen, die keine Splittung der Stichprobe zur Gruppenbildung benötigen, parametrische Verfahren eingesetzt werden können (Bortz \& Schuster, 2010). Die Darstellung der Daten zur Maßnahme Schulbegleitung in Stadt und Landkreis Göttingen erfolgt überwiegend deskriptivstatistisch. Zur Wahrung der Übersichtlichkeit werden die Häufigkeiten bestimmter Angaben teilweise gruppiert präsentiert. Zur Hypothesenprüfung hinsichtlich der Kooperationspraxis mit der Klassenleitung und den Kooperationswirkungen kommen inferenzstatistische Verfahren zum Einsatz. Die Untersuchung der Zusammenhänge von kooperationsspezifischen Merkmalen mit Daten zu den Schulbegleiter/innen oder zur Maßnahme erfolgt je nach Fallzahl und Datenstruktur auf deskriptivem Niveau oder anhand inferenzstatischer Analysen.

38 Die fehlende Angabe einer Person, die den Papierfragebogen ausgefüllt hatte, bei der Frage, ob eine Berufsausbildung abgeschlossen wurde, konnte aufgrund der weiteren Angaben der Person ergänzt werden. 


\section{Ergebnisse}

Im Folgenden werden zunächst die Ergebnisse der deskriptiv angelegten Abfragen zu Schulbegleitung präsentiert. Anschließend erfolgen die Analysen zu Einbindung und Kooperation und die Untersuchung möglicher Zusammenhänge zwischen den deskriptiven Daten und den Daten zu Kooperation (siehe Forschungsfragen). Zur besseren Einordnung der erfassten Zahlen zu Schulbegleitung erfolgt an dieser Stelle eine kurze Übersicht über die aktuelle Göttinger Schullandschaft. Die Daten entstammen den Internetseiten der Stadt Göttingen (Referat Statistik und Wohnen) und des Niedersächsischen Landesinstituts für schulische Qualitätsentwicklung sowie den Angaben von Krämer-Mandeau und Scharnhorst-Engel (2014). Die Schülerschaft in Stadt und Landkreis Göttingen verteilt sich auf 62 Grundschulen, vier Hauptschulen, vier Realschulen, vier Oberschulen, sieben Gymnasien, sechs Gesamtschulen (davon vier Integrierte Gesamtschulen), acht Förderschulen und elf Berufsschulen. Mit Ausnahme der Förderschwerpunkte Hören und Sehen decken die Förderschulen zusammengenommen alle Förderschwerpunkte (Lernen, Sprache, Emotionale und soziale Entwicklung, Geistige Entwicklung, Körperliche und motorische Entwicklung) ab. Die Schülerschaft an den Förderschulen ist überwiegend männlich. Die Schülerzahl in Stadt und Landkreis beträgt insgesamt 25089 (14 016 in der Stadt Göttingen und 11073 im Landkreis Göttingen). Nimmt man als Vergleichswert die amtliche Gesamtzahl an Schulbegleitungen zum Jahresende 2015 (215), kommt auf etwa 117 Schüler/innen eine Schulbegleitung. ${ }^{39}$

Nicht nur den Einschätzungen der Träger nach, auch laut Auskunft der Ämter hat sich die Zahl an Schulbegleitungen stark erhöht. Genaue Fallzahlen konnten vom Jugendamt Stadt und vom Sozialamt Landkreis erfragt werden (siehe Abbildung 3). Im Jahr 2003 wurde über das Jugendamt Stadt eine Maßnahme bewilligt, seitdem sind die Fallzahlen jährlich angestiegen.

39 Da der Wohnort und nicht der Schulort des Kindes oder Jugendlichen darüber entscheidet, welches Amt zuständig ist, kann das Verhältnis nur geschätzt werden. 


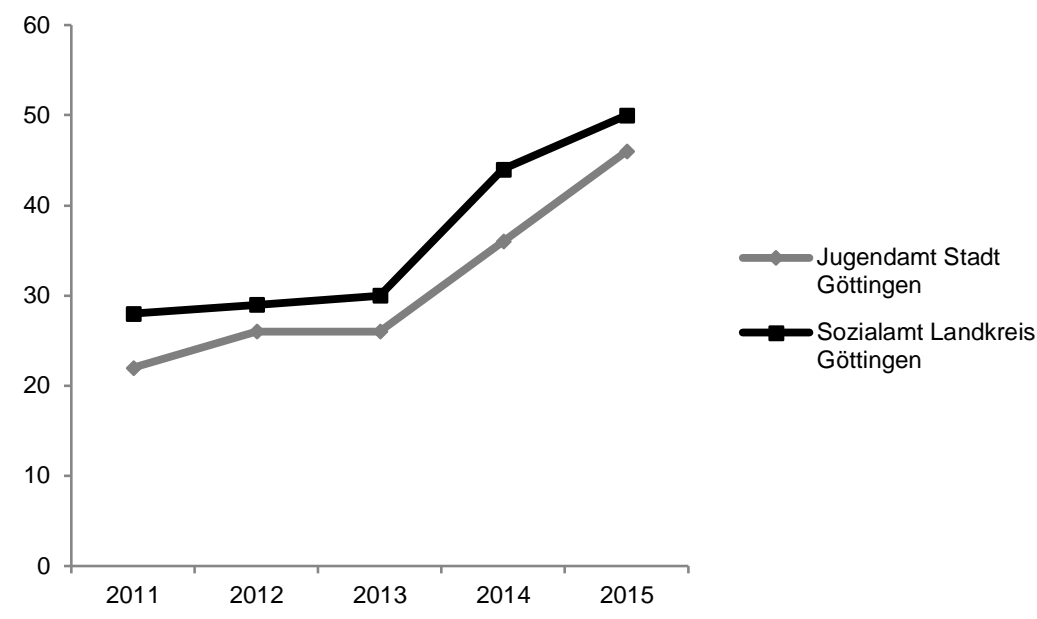

Abb. 3: Entwicklung der Bewilligungszablen von Schulbegleitungen durch das Jugendamt der Stadt und das Sozialamt des Landkreises Göttingen

\subsection{Rahmendaten zu Schulbegleitung in Stadt und Landkreis Göttingen}

Die Ergebnisse werden an dieser Stelle entsprechend der Reihenfolge der Forschungsfragen (siehe Kapitel 5), die weitgehend auch der Reihenfolge im Fragebogen entspricht, vorgestellt. Zunächst werden die Angaben zur Person und Qualifikation berichtet, darauf die Daten zum Beschäftigungsverhältnis, zu den begleiteten Schüler/innen und zu den ausgeübten Tätigkeiten.

\subsubsection{Person und Qualifikation}

Von den befragten Schulbegleiter/innen geben 41 weiblich als Geschlecht an (87.2\%) und $6(12.8 \%)$ männlich. ${ }^{40}$ Die Altersangaben streuen zwischen 24 und 61 Jahren, der Schnitt liegt bei 45 Jahren. Sämtliche Befragte verfügen über einen Schulabschluss, $31(62.8 \%)$ von ihnen geben Abitur oder Fachabitur/Fachhochschulreife als höchsten Schulabschluss an, 13 (27.7\%) einen Realschulabschluss oder vergleichbare Abschlüsse und 3 (6.4\%) einen Hauptschulabschluss oder vergleichbare Abschlüsse.

Insgesamt geben 38 Personen (80.1\%) an, mindestens eine Ausbildung/ein Studium abgeschlossen zu haben (32 bzw. 68.1\%) oder sich in Ausbildung/Studium zu befinden (6 bzw. 12.8\%), während auf 9 Personen (19.1\%) weder das eine noch das andere zutrifft. Drei Befragte, die zuvor Ausbildung oder Studium angegeben hatten, haben hierfür keine Auswahl getroffen, d. h. die Zahlen derjenigen, die sich in Ausbildung/Studium befinden oder dies abgeschlossen haben, liegen noch geringfügig höher. Nähere Datenanalysen ergeben, dass drei Personen sowohl eine Ausbildung als auch ein Studium abgeschlossen haben und drei weitere Personen sich in ihrer zweiten Ausbildung bzw. einem Studium nach abgeschlossener Ausbildung befinden. ${ }^{41}$ Die Fachkraftquote, also der Anteil derer, die eine Ausbildung oder ein Studium mit pädagogischen oder pflegerischen Anteilen abgeschlossen haben, beträgt 44.7\% $(n=21)$. Eine Übersicht darüber, zu welchen Anteilen einschlägige formale Qualifikationen vorliegen, bietet Abbildung 4 .

40 Die weiteren Antwortkategorien („,Ein anderes“ und „Keine Angabe“) wurden nicht ausgewählt.

41 Dabei wurden die Angaben manuell geprüft; wurden z. B. sowohl Erziehungswissenschaften als auch „Sonstiges Studium“ markiert, zählt dies nur als ein Studium, da die Kombination von zwei Fächern in einem Studiengang üblich ist (das Absolvieren zweier Studiengänge dagegen nicht). Genauso wurde z. B. verfahren, wenn „Sonderpädagogik“" und „Lehramtsstudium“ ausgewählt wurden, da Sonderpädagogik üblicherweise im Rahmen eines Lehramtsstudiums studiert wird. 

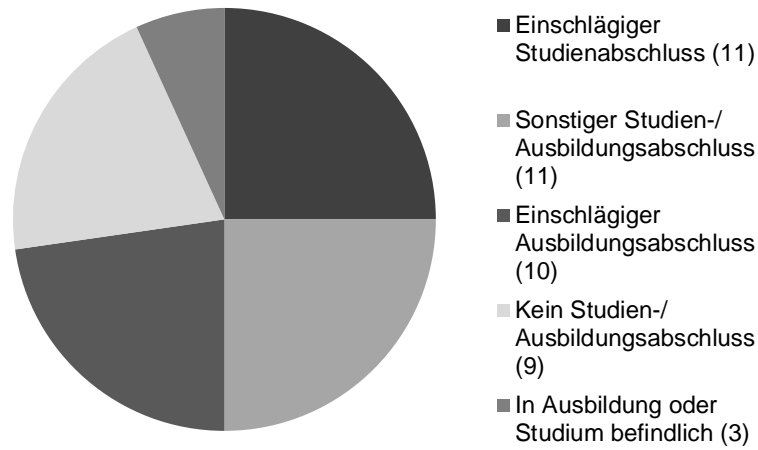

Abb. 4: Anteilige Verteilung der Schulbegleiter/ innen (jeweiliges $\mathrm{n}$ in Klammern) nach höchstem einscblägigen formalen Bildungsabschluss (Pädagogik oder Pflege; hat eine Person ₹. B. eine einschlägige Ausbildung und ein sonstiges Studium absolviert, wird sie der ersten Gruppe zugeordnet)

Hinsichtlich der Bereiche, in denen man schon vor der Tätigkeit als Schulbegleitung Erfahrung hatte, ist das berufliche Arbeiten mit Kinder oder Jugendlichen die häufigste Angabe $(n=33,70.1 \%)$, gefolgt von der Erziehung eigener Kinder oder Pflegekinder $(n=28,59.6 \%)$ und der ehrenamtlichen Arbeit mit Kindern oder Jugendlichen $(n=23,48.9 \%)$. Vier Personen $(8.5 \%)$ gaben bei sonstigen Erfahrungen berufliche oder private Tätigkeiten an, die sich nicht den vorher genannten Kategorien zuordnen lassen (z. B. die Arbeit in der Erwachsenenbildung). Das offene Antwortformat wurde außerdem von einigen zur genaueren Angabe, welche berufliche Tätigkeit mit Kindern oder Jugendlichen ausgeübt wurde, genutzt, wobei die genannten Tätigkeiten überwiegend dem sozialpädagogischen Bereich zugehören. Zwei Befragte (4.3\%) gaben an, zuvor noch keine Erfahrung mit Kindern oder Jugendlichen gehabt zu haben.

Es folgen Angaben, die enger auf die Tätigkeit als Schulbegleitung bezogen sind. Zunächst werden an dieser Stelle die Zeitangaben zur Beschäftigung als Schulbegleitung dargestellt. Im Schnitt sind die Befragten seit 2 Jahren und 8 Monaten $(S D \approx 2$ Jahre und 4 Monate) als Schulbegleitung tätig, wobei der unterste Wert 4 Monate beträgt und der höchste Wert 8 Jahre und 10 Monate. Die Frage danach, wie lange sie, unabhängig von der aktuellen Vertragsdauer, noch als Schulbegleitung tätig sein möchten, wurde von den Befragten mit Angaben zwischen 0 Monaten $(n=9)$ und 40 Jahren $(n=1)$ beantwortet, der Schnitt lag bei 5 Jahren und 7 Monaten ( $S D \approx 7$ Jahre und 6 Monate). Eine Korrelationsanalyse für beide Abfragen ergibt einen signifikanten, positiven, kleinen Zusammenhang $(r=.34, p<.05)^{42}$. Eine vergleichende, gruppierte Übersicht findet sich in Abbildung 5.

42 Der Ausreißerwert von 40 Jahren blieb bei der Berechnung unberücksichtigt, da er verzerrend auf den Korrelationskoeffizienten wirken kann (Sedlmeier \& Renkewitz, 2013). 


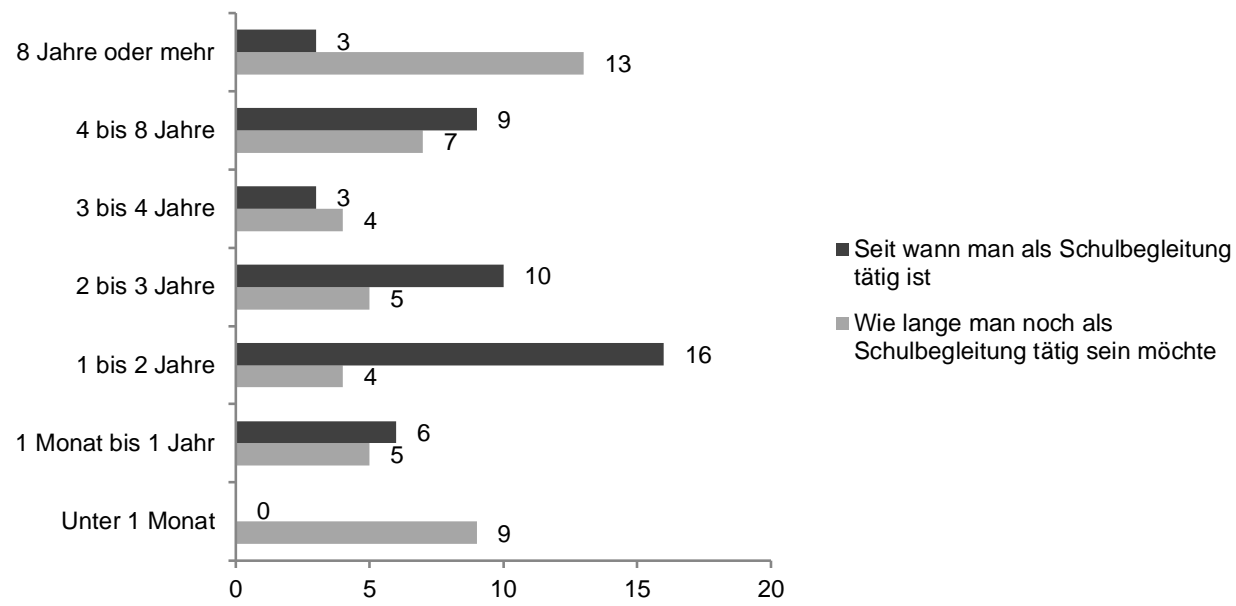

Abb. 5: Häufigkeiten der Zeitangaben zur bisherigen Beschäftigungsdauer als Schulbegleitung und zur gewünschten weiteren Beschäftigungsdauer (Angaben, die genau eine Jabreszabl treffen, sind jeweils der höheren Kategorie zugeordnet; ₹. B. sind 24 Monate der Kategorie 2 bis 3 Jahre zugeordnet)

Die Frage, aus welchen Gründen man Schulbegleiter/in geworden ist, wurde auf einer Ratingskala beantwortet (trifft gar nicht $\mathfrak{z}$ - trifft eher nicht $z u$ - trifft eher $₹ u$ - trifft voll $₹ u$; Kodierung von 1 bis 4). Auffällig ist eine Häufung von fehlenden Angaben bei einzelnen Befragten. Deshalb werden an dieser Stelle bei jedem Item die fehlenden Werte (missings) angegeben und bei den (gültigen) Prozentangaben wird in Klammern auch die Prozentzahl bezogen auf alle Befragten aufgeführt. ${ }^{43}$ Die höchste Zustimmung erhält die Antwortvorgabe „Weil ich gerne mit Kindern/Jugendlichen zusammenarbeite“ $(M=3.76, S D=.57$, missings $=1)$. Bis auf eine Person bewerten alle Befragten, d. h. 97.8\%, diese Aussage als (eher) zutreffend (78.7\%). Auch die spezifizierte Aussage „Weil ich gerne mit Kindern/Jugendlichen mit Förderbedarf arbeite“ erhält eine ähnlich hohe mittlere Zustimmung $(M=3.58, S D=.64$, missings $=4)$, ebenso die Aussagen „Weil die Arbeitszeiten für mich günstig sind“ $(M=3.64, S D=.62$, missings $=5)$ und ,Weil ich gerne im Bereich Schule arbeite" $(M=3.4, S D=.69$, missings $=4)$. Genau dem theoretischen Skalenmittelpunkt von 2.5, d. h. einer neutralen Position zwischen Zustimmung und Ablehnung, entspricht der Mittelwert für „Weil ich mich beruflich neu orientieren möchte“ $(S D=.7$, missings $=5)$. Darunter liegen die Mittelwerte für „Weil ich in meinem Wunsch-Tätigkeitsfeld keine Arbeit gefunden habe“ $(M=2.13, S D=.96$, missings $=9)$ und „Weil ich für Schulbegleitung keine formale Qualifikation brauche" $(M=1.93, S D=.98$, missings $=6)$. Als sonstige Gründe, die im freien Format eingegeben werden konnten, sind zwei generelle nützlichkeitsorientierte Gründe angegeben, zwei auf Arbeitsinhalte bezogene und zwei, die beide Aspekte beinhalten. Im Schnitt erlangen die Gründe, die sich auf die Arbeitsinhalte beziehen, höhere Zustimmungswerte als die Nützlichkeitsgründe. Unter den Nützlichkeitsgründen stehen die Arbeitszeiten an erster Stelle, die Zustimmungsrate (d. h. der Anteil derer, die das Item mit trifft eher $₹ u$ oder trifft voll $z u$ beantworten) beträgt hierbei 92.4\% (82.9\%). Demgegenüber fallen die Zustimmungsraten für Nützlichkeitsgründe, die in Zusammenhang mit der formal niedrigen Einstiegsschwelle der Tätigkeit stehen, geringer aus: Für die berufliche Neuorientierung beträgt die Zustimmungsrate $54.8 \%$ (49\%), für die Aussage, man habe im Wunschtätigkeitsfeld keine Arbeit gefunden, $36.8 \%(29.4 \%)$ und für die Nichtnotwendigkeit einer formalen Qualifikation $24.4 \%(21.3 \%)$.

\subsubsection{Beschäftigungsverhältnis}

Den Angaben der Befragten nach werden 73.8\% der Maßnahmen über die Jugendämter bewilligt, 40.5\% $(n=17)$ über das Jugendamt Stadt und 33.3\% $(n=14)$ über das Jugendamt Landkreis. Über die Sozialämter

43 Fast alle dieser Befragten geben bei den Items, die sie bearbeitet haben, hohe Zustimmungswerte an. Vorstellbar ist deshalb, dass der Grund für die fehlenden Angaben im Antwortformat liegen könnte: Zwar wird in der Frageinstruktion explizit darum gebeten, zu jeder Aussage eine Antwort anzukreuzen, die Befragten sind an dieser Stelle aber zum ersten Mal im Fragebogen mit einer Ratingskala konfrontiert. Möglicherweise sind einige Befragte der Logik der bisherigen Antwortformate gefolgt und haben nur bei den Gründen, die auf sie zutreffen, ein Kreuzchen gesetzt. Wenn dem so ist, sind die Mittelwerte künstlich erhöht und bei den Zustimmungsraten die Gesamtprozente aussagekräftiger als die gültigen Prozente. 
laufen 26.1\% der Maßnahmen, wobei in diesem Fall deutlich mehr Maßnahmen vom Sozialamt Landkreis ausgehen $(19 \%, n=8)$ als vom Sozialamt Stadt $(7.1 \%, n=3)$. Fünf Befragte geben an, den Kostenträger nicht zu wissen oder gaben einen freien Träger als Kostenträger ${ }^{44}$ an, diese Angaben bleiben bei den genannten Prozentangaben unberücksichtigt. Sämtliche Befragte $(n=47)$ geben an, über einen freien Träger angestellt zu sein. 45 Über Dreiviertel $(76.6 \%, n=36$ ) arbeiten auf Angestelltenbasis, ein knappes Viertel $(23.4 \%, n=11)$ auf Honorarbasis. Die Antwortoptionen „FSJ/BFD“ und „Ehrenamt“ wurden nicht ausgewählt. Die Laufzeit des aktuellen Vertrags als Schulbegleitung schwankt zwischen dem Minimum von einem Monat und dem Maximum von 2 Jahren und 3 Monaten. Die mit Abstand häufigste Vertragslaufzeit sind 12 Monate $(45 \%, n=21)$, der Schnitt knapp 11 Monate $(S D \approx 4$ Monate). Nur 3 Verträge laufen über einen längeren Zeitraum als 12 Monate. Eine gruppierte Übersicht findet sich in Abbildung 6 .

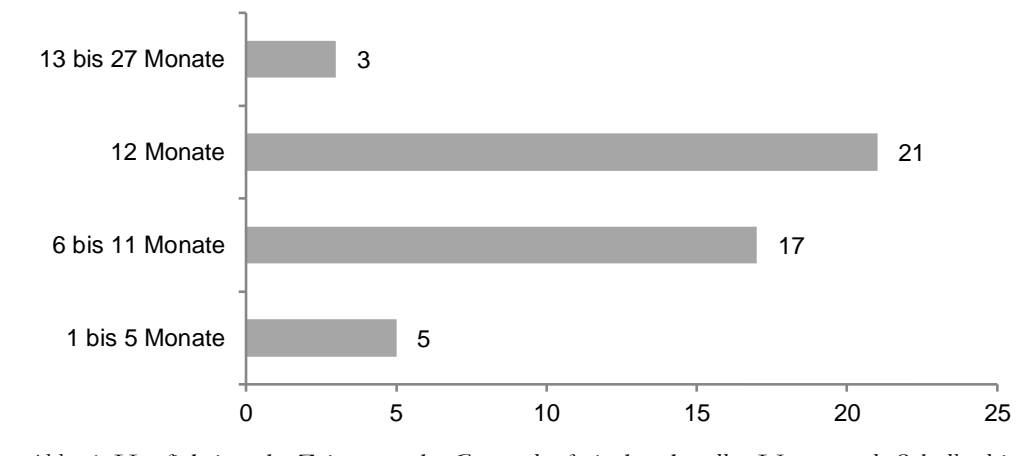

Abb. 6: Häufigkeiten der Zeiträume der Gesamtlaufzeit des aktuellen Vertrags als Scbulbegleitung

Die Zufriedenheitswerte hinsichtlich bestimmter Aspekte des Arbeitsverhältnisses fallen durchwachsen aus. Mit Ausnahme der vergleichsweise positiven Bewertung der Urlaubsregelungen liegen die Werte im Schnitt jeweils etwas unter dem theoretischen Skalenmittelwert von 2.5, der eine neutrale Position (weder zufrieden noch unzufrieden) bedeutet. Aus den einzelnen Items lässt sich, auch bei Herausnahme der am wenigsten korrelierenden Items, keine Skala mit akzeptablen Kennwerten bilden. Offensichtlich hängen die Zufriedenheitswerte für die einzelnen Aspekte also nicht oder nur bedingt zusammen. Mit der Entlohnung sind 31 Befragte $(66 \%)$ eher oder sehr unzufrieden $(M=2.28, S D=.83)$, mit der Laufzeit des Vertrags 25 (55.8\%, $M=2.31, S D=.92)$ und mit den Regelungen im Krankheitsfall $26(56.5 \%, M=2.24, S D=.92)$. Mit den Urlaubsregelungen ist die große Mehrheit $(n=37,80.4 \%, M=2.93, S D=.83)$ dagegen eher oder sehr zufrieden.

Von den Befragten geben $10(21.3 \%)$ an, dass ihr Arbeitgeber keine Schulungen anbiete. Die große Mehrheit $(78.7 \%, n=37)$ arbeitet aber bei Trägern, die Schulungen anbieten. Insgesamt haben $11(23.4 \%)$ der Befragten bereits an einer solchen Schulung und 17 (36.2\%) bereits an mehr als einer solchen Schulung teilgenommen. Weitere 9 der Befragten (19.1\%) haben das Schulungsangebot noch nicht wahrgenommen.

Nur 2 der 46 Schulbegleiter, die zu dieser Frage Angaben machen (eine fehlende Angabe), sind für mehr als eine/n Schüler/in als Schulbegleitung tätig. Den Schüler oder die Schülerin, für den oder die sie aktuell hauptsächlich tätig sind, begleiten die Befragten im Schnitt seit $1 \mathrm{Jahr}$ und 6 Monaten $(\mathrm{SD} \approx 1 \mathrm{Jahr}$ und 4 Monate), die kürzeste Dauer beträgt 2 Monate und die längste 6 Jahre. Für eine gruppierte Übersicht siehe Abbildung 7.

44 Vermutlich liegt hier entweder ein Missverstehen der Frage vor oder die Unkenntnis über die Zweiteilung Kostenträger vs. ausführender Träger.

$45 \mathrm{Zu}$ beachten ist dabei der Stichprobenzugang: Außer bei den Schulbegleiter/innen, die im Rahmen einer Maßnahme des Jugendamts der Stadt arbeiten, erfolgte die Kontaktaufnahme ausschließlich über die freien Träger. 


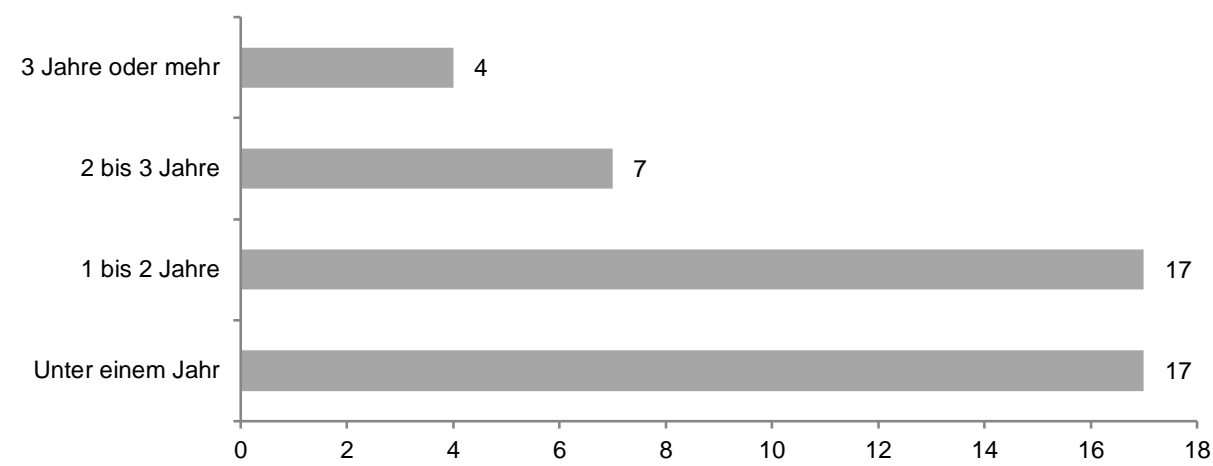

Abb. 7: Häufigkeiten der Zeitangaben zur bisherigen Tätigkeit für den aktuell begleiteten Scbüler oder die aktuell begleitete Scbülerin

\subsubsection{Begleitete Schüler/innen}

Bei über der Hälfte der begleiteten Schüler/innen $(57.8 \%, n=26)$ wurde den Angaben der Befragten nach Schulbegleitung aufgrund einer seelischen Behinderung beantragt, bei etwa einem Drittel $(35.6 \%, n=16)$ aufgrund geistiger Behinderung und bei weniger als einem Zehntel $(6.7 \%, n=3)$ aufgrund körperlicher Behinderung. Den Angaben nach, welches Amt die Kosten trägt, liegt der Anteil seelischer Behinderungen höher und der Anteil geistiger oder körperlicher Behinderungen niedriger. Abbildung 8 stellt beide Angaben vergleichend dar.
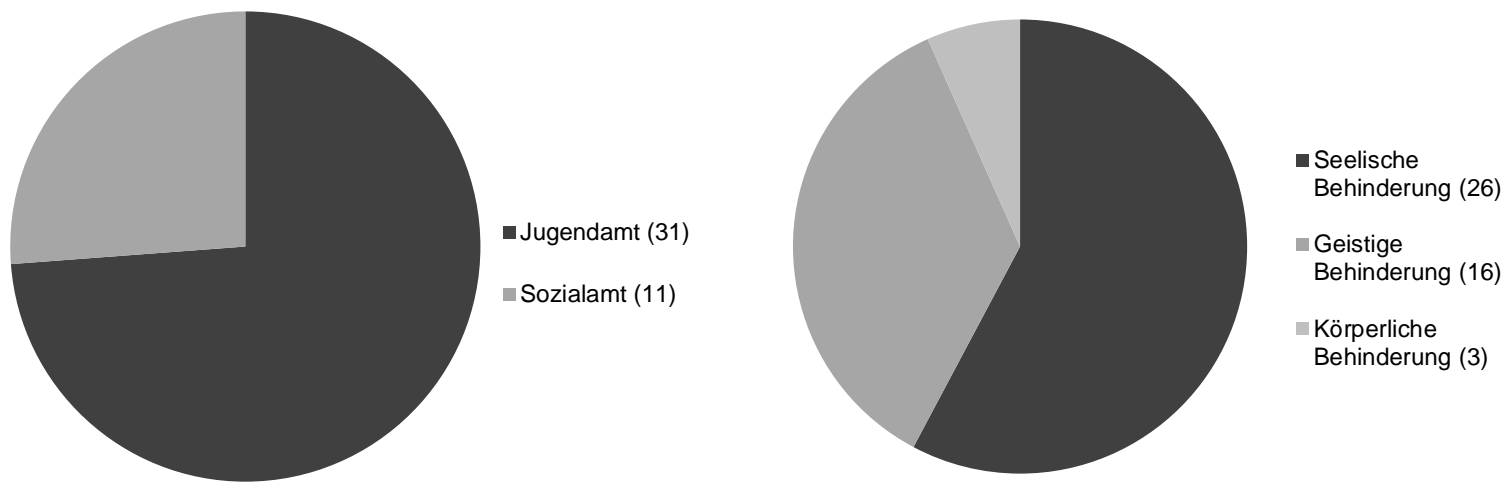

Abb. 8: Bewilligendes Amt der Maßnabme (links) und Angabe der Bebinderung der Scbüler/innen durch die Schulbegleiter/innen (n in Klammern)

Bei den Schulformen (für einen Überblick siehe Abbildung 9) dominiert mit großem Abstand die Grundschule $(56.5 \%, n=26)$, gefolgt von den Gesamtschulen $(19.6 \%, n=9)$. In den anderen weiterführenden Schulen und in Förderschulen arbeiten jeweils nur wenige Schulbegleiter/innen, an Kollegs/Abendgymnasien und Berufsschulen gar keine. 


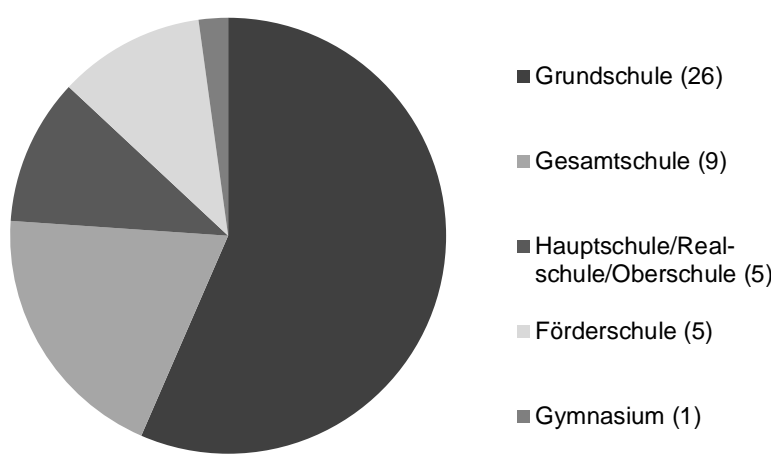

Abb. 9: Verteilung der Schulbegleitungsmaßnabmen über die Schulformen (n in Klammern; die Optionen „Kollegs/Abendgymnasium" und „Berufsscbule" wurden nicht ausgewäblt)

Im Schnitt sind die Befragten 24 Stunden pro Woche ( $S D \approx 8$ Stunden) für ihre/n Schüler/in als Schulbegleitung tätig bei Angaben zwischen 4 Stunden und 40 Stunden. Der häufigste Stundenumfang beträgt 25 $(n=13$, das entspricht 28.3\%). Eine gruppierte Übersicht findet sich in Abbildung 10.

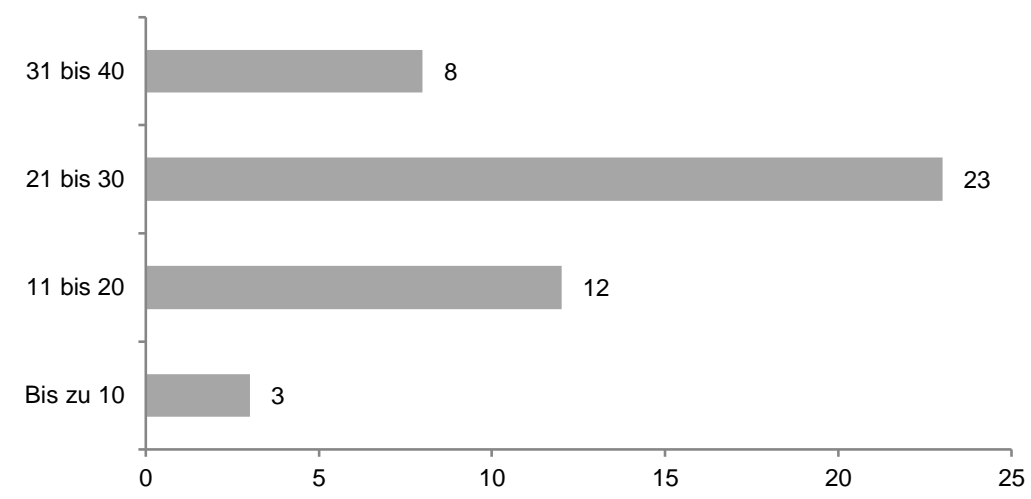

Abb. 10: Häufigkeiten der jeweiligen Wochenstunden der Scbulbegleitung

Bezüglich der Unterrichtsformen während der begleiteten Schulstunden sollten Häufigkeitseinschätzungen abgegeben werden (nie - selten - manchmal - oft - sehr oft, Kodierung von 1 bis 5). Es dominieren Einzelarbeit und Frontalunterricht, gefolgt von Partnerarbeit und Gruppenarbeit. Einzelarbeiten finden nach Einschätzung von 37 Befragten $(84.1 \%$ ) oft oder sehr oft statt (und nur bei $n=1$ seltener als manchmal; $M=4.05$, $S D=.68)$, für lehrerzentrierten Frontalunterricht teilen 27 der Befragten $(61.3 \% ; M=3.68, S D=1.2)$ diese Einschätzung. Partnerarbeiten werden demnach bei 17 Befragten $(38.6 \% ; M=3.36, S D=.94)$ oft oder sehr oft eingesetzt und Gruppenarbeiten bei 11 Befragten $(25 \% ; M=3.17, S D=.95)$. Für alle genannten Unterrichtsformen geben weniger Befragte (zwischen $n=1$ und $n=8$ ) die Häufigkeiten nie oder selten an als die Häufigkeiten oft oder sehr oft, so dass insgesamt von einer Methodenvariation ausgegangen werden kann. In den einzelnen Fällen kann diese verschiedentlich ausfallen; bivariate Zusammenhangsanalysen ergeben nur für den Einsatz von Gruppenarbeit und Partnerarbeit einen signifikanten, mittleren, positiven Zusammenhang $(r=.67, p<.001)$. Bei $12(26.1 \%)$ der befragten Schulbegleitungen ist noch eine weitere Schulbegleitung in der gleichen Klasse tätig. Eine Koordinationskraft für alle Schulbegleiter/innen der Schule existiert bei 33 Schulbegleiter/innen ( $71.7 \%)$. 


\subsubsection{Tätigkeitsprofil}

Auch die Tätigkeiten, die im Rahmen der Schulbegleitung ausgeübt werden, sollten auf einer Häufigkeitsskala (nie - selten - manchmal - oft - sehr oft, Kodierung von 1 bis 5) eingeschätzt werden. Bevor zusammenfassende Werte für Teilbereiche ermittelt werden, erfolgt an dieser Stelle die differenzierte Analyse der einzelnen Tätigkeiten. Für eine anschauliche gruppierte Übersicht (die aber nicht die einzelnen Werte enthält) siehe Abbildung 11.

Im Bereich lebenspraktischer Anforderungen gibt eine Mehrheit von 74.4\% $(n=32)$ an, Tätigkeiten in der Pflege und Mobilitätshilfe nie auszuüben, nur eine Minderheit von $9.4 \%(n=4)$ tut dies oft oder sehr oft ( $M=1.6, S D=1.16)$. Bei der Unterstützung bei der Verwendung von Hilfsmitteln verteilen sich die Häufigkeitsangaben gleichmäßiger über die Antwortmöglichkeiten $(M=2.93, S D=1.45)$, ebenso bei der Orientierungshilfe (z. B. vom Bus abholen, Raumwechsel begleiten; $M=3.39$, $S D=1.47$ ). Die Beaufsichtigung während der Pause übernehmen 36 der Befragten (82.6\%) oft oder sehr oft $(M=4.26, S D=0.85)$. Hinsichtlich der Aufgaben, die dem Bereich der Emotions- und Verhaltensregulation zugeordnet sind, geben bis auf eine Ausnahme alle Befragten an, den Schüler oder die Schülerin oft oder sehr oft bei der emotionalen Regulierung zu unterstützen (z. B. beruhigen, ermuntern) ( $n=46$, also 97.8\%; $M=4.63, S D=.53$ ). Die große Mehrheit unterstützt oft oder sehr oft Sozialkontakte zu anderen Schüler/innen $(n=40,86.9 \%$; $M=4.26, S D=.93$ ) und interveniert oft oder sehr oft bei (auto)aggressivem Verhalten bzw. bei Regelverstößen (jeweils $n=32,69.6 \% ; M=3.93, S D=1.24$ bzw. $M=3.93$, $S D=1.2$ ). Auch Aufgaben im didaktischen Bereich werden generell häufig übernommen. Im Frontalunterricht üben 7 der Befragten (15.2\%) nie oder selten unterstützende Tätigkeiten wie die Wiederholung oder Erklärung des von der Lehrkraft Gesagten aus, $30(65.3 \%)$ dagegen oft oder sehr oft $(M=3.91, S D=1.23)$. In Gruppenarbeitsphasen bzw. Einzelarbeitsphasen leisten nur $4(8.8 \%)$ bzw. $2(4.3 \%)$ nie oder selten Unterstützung (z. B. durch Erklärung der Aufgaben und Hilfe bei der Lösungssuche) und 32 (71.1\%) bzw. $38(82.6 \%)$ oft oder sehr oft $(M=3.96$, $S D=1.09$ bzw. $M=4.26, S D=.85$ ). Die befragten Schulbegleiter/innen unterstützen häufig auch andere Schüler/innen im Unterricht, wobei die Angaben hier insgesamt geringer ausfallen als für die Unterstützung der eigenen Schülerin oder des eigenen Schülers; 10 Befragte (23.3\%) tun dies nie oder selten und 19 (44.2\%) oft oder sehr oft $(M=3.28, S D=1.05)$.

Die Möglichkeit, bis zu drei weitere Tätigkeiten frei einzugeben, wurde von insgesamt 11 Teilnehmenden genutzt um insgesamt 23 Tätigkeiten anzugeben, die sie oft oder sehr oft (in wenigen Fällen: manchmal) ausführen. Einige Tätigkeiten lassen sich den zuvor aufgeführten Antwortmöglichkeiten zuordnen (z. B. Schulbusbegleitung zu „Orientierung geben“ und Unterstützung beim Essen zu „Pflege“). Darüber hinaus wurden 12 Tätigkeiten genannt, die sich auf unterrichtsbezogene Unterstützung beziehen, ohne klar einem der vorgegeben Unterrichtsformate zuordenbar zu sein (z. B. Arbeitsmaterial bereitlegen, Erlerntes wiederholen und üben, Führen des Hausaufgabenheftes, Unterstützung des Lehrpersonals, Hausaufgaben kontrollieren). Zudem wurden 6 Tätigkeiten angegeben, die sich auf die Kooperation mit verschiedenen Personengruppen (Lehrkräften, Eltern, Therapeut/innen, Arbeitgeber/innen) beziehen. 


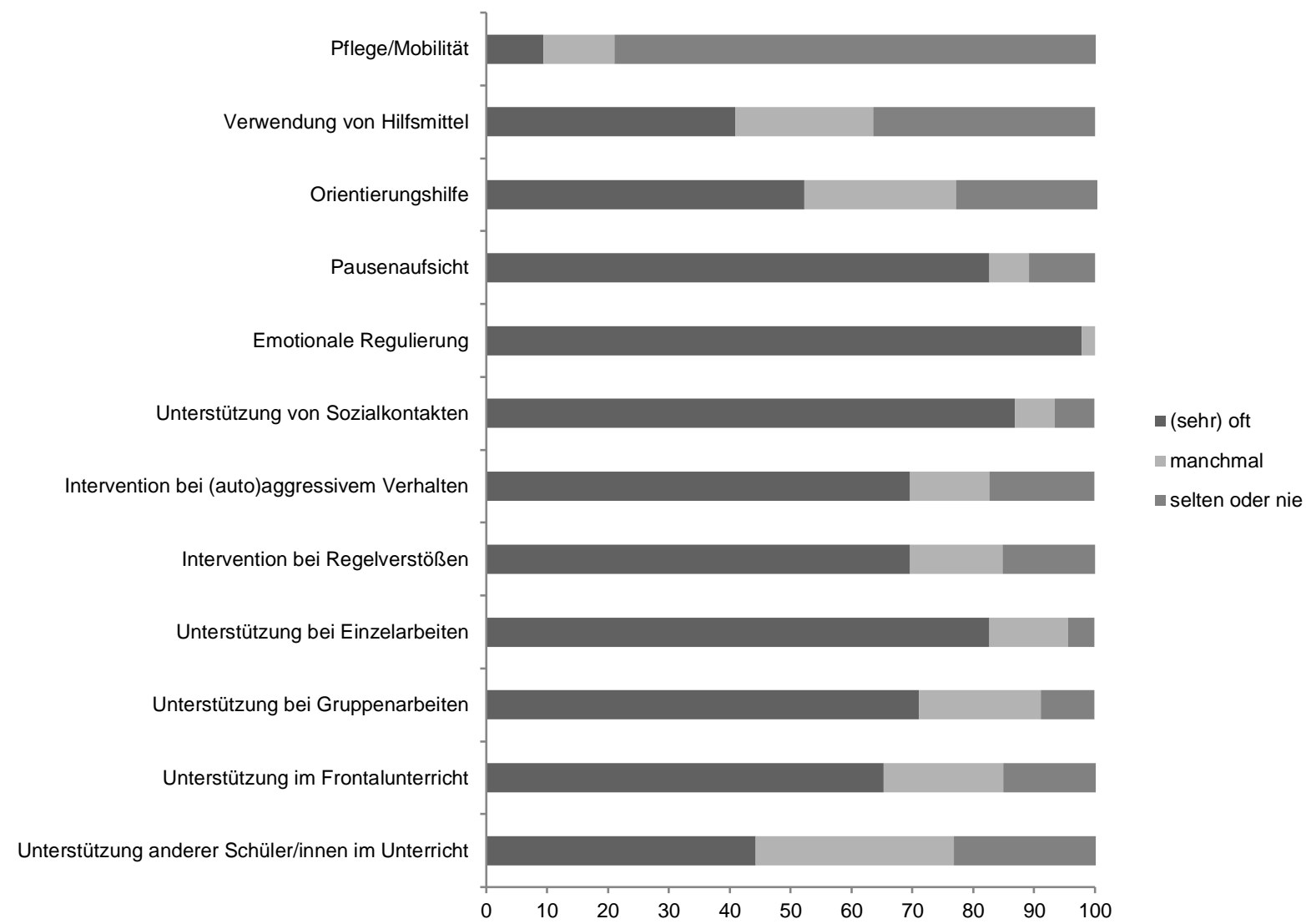

Abb. 11: Prozentuale Nennung (nur gültige Prozente) der Häufigkeit der Ausübung von Tätigkeiten im Rabmen von Schulbegleitung

Bei der vergleichenden Betrachtung der Häufigkeitsangaben fällt auf, dass Tätigkeiten im Bereich lebenspraktischer Anforderungen einen geringeren Raum einnehmen als didaktische Unterstützung und Tätigkeiten im Bereich der Emotions- und Verhaltensregulation. Da die Stichprobengröße keine Faktorenanalyse zulässt, wurde mittels Korrelationsanalysen geprüft, ob sich die einzelnen Tätigkeiten so zusammenfassen lassen, dass eine Berechnung von Durchschnittswerten für die zuvor definierten Aufgabenbereiche Lebenspraktische Anforderungen, Emotions- und Verbaltensregulation und Didaktische Unterstützung möglich ist. Eine Zusammenfassung erschien vor allem im Hinblick auf die spätere Analyse von Zusammenhängen der Tätigkeiten mit weiteren Merkmalen der Schulbegleitungsmaßnahme sinnvoll. Mit Ausnahme der Pausenaufsicht und der unterrichtsbezogenen Unterstützung anderer Schüler/innen korrelieren die Häufigkeiten der Tätigkeiten, die demselben Aufgabenbereich zugeordnet waren, jeweils signifikant positiv $(\phi<.05) .{ }^{46}$ Die Pausenaufsicht korreliert nicht mit den Tätigkeiten im Bereich lebenspraktischer Anforderungen, dafür aber signifikant positiv $(p<.05)$ mit den Tätigkeiten aus dem Bereich der Emotions- und Verhaltensregulation. Die Häufigkeit unterrichtsbezogener Unterstützung anderer Schüler/innen lässt sich keinem Bereich zuordnen. Sie korreliert lediglich mit der Häufigkeit der Unterstützung in Gruppenarbeitsphasen $(r=.37$, $p<.05)$ und (negativ) mit der Pausenaufsicht $(r=-.37, p<.05)$. Auf der Basis dieser Inter-Item-Korrelationen wurden Skalenanalysen (Skalen Lebenspraktische Anforderungen, Emotions- und Verbaltensregulation, Didaktische Unterstützung) durchgeführt, wobei die Pausenaufsicht der Skala Emotions- und Verhaltensregulation zugeordnet wurde ${ }^{47}$ und die unterrichtsbezogene Unterstützung anderer Schüler/innen unberücksichtigt blieb. Die Werte für die Trennschärfe der Items (zwischen $r_{i t}=.38$ und $r_{i t}=.76$ ) und für die Reliabilität der Skalen (jeweils $a>.7$ ) liegen jeweils auf akzeptablem Niveau.

46 Darüber hinaus legen die Werte der Inter-Item-Korrelationen nahe, dass ein höherer Bedarf an Unterstützung in einem Bereich tendenziell mit einem höheren Bedarf an Unterstützung auch in einem anderen Bereich einhergeht. Lediglich Aufgaben im Bereich Pflege/Mobilität hängen ausschließlich mit anderen Aufgaben im Bereich lebenspraktischer Anforderungen zusammen.

47 Tatsächlich kann Pausenaufsicht sowohl Hilfe bei lebenspraktischen Anforderungen als auch Hilfe bei der Emotions- und Verhaltensregulation beinhalten; in dieser Stichprobe scheint letzteres der Fall zu sein. 
Die Skalenmittelwerte zeigen, dass die Tätigkeiten im Bereich Emotions- und Verhaltensregulation im Schnitt den größten Raum einnehmen $(M=4.2, S D=.76)$, gefolgt von Tätigkeiten im Bereich didaktischer Unterstützung $(M=4.1, S D=.92)$. Weitaus weniger Raum nehmen im Schnitt Tätigkeiten im Bereich lebenspraktischer Anforderungen ein $(M=2.66, S D=1.12)$. Die Skala Didaktische Unterstützung korreliert sowohl mit der Skala Lebenspraktische Anforderungen $(r=.38, p<.05)$ als auch mit der Skala Emotionsund Verhaltensregulation $(r=.34, p<.05)$. Die Skalen Lebenspraktische Anforderungen und Emotionsund Verhaltensregulation hängen nicht zusammen.

\subsubsection{Zusammenhangsanalysen}

Im Folgenden sollen die im ersten Fragebogenteil erhobenen Daten auf Zusammenhänge untersucht werden. Dabei werden ausschließlich Analysen vorgenommen, die inhaltlich-theoretisch hergeleitet sind und sich an den Forschungsfragen (siehe Kapitel 5) orientieren. Wegen der teilweise geringen Fallzahlen wird auf inferenzstatistische Analysen in den meisten Fällen verzichtet, so dass hauptsächlich deskriptive Werte berichtet werden. Zur Analyse von Zusammenhängen der Qualifikation der Befragten mit weiteren Merkmalen wurden vier Gruppen gebildet: Fachkräfte (einschlägiger Ausbildungs- oder Studienabschluss), Nichtfachkräfte mit sonstigem Ausbildungs- oder Studienabschluss, Nichtfachkräfte ohne Abschluss (die auch nicht in Ausbildung oder Studium befindlich sind), Nichtfachkräfte in Ausbildung oder Studium (vgl. Abbildung 4). Der Zusammenhang von Qualifikation und Motivation der Schulbegleiter/innen interessiert vor dem Hintergrund, dass Schulbegleitung keine formale Qualifikation voraussetzt. Zwei Items wurden speziell vor diesem Hintergrund formuliert, nämlich „Weil ich für Schulbegleitung keine formale Qualifikation brauche“ und „Weil ich mich beruflich neu orientieren möchte“. Im ersten Fall liegt der Mittelwert der Fachkräfte $(M=1.33, S D=1.01)$ deutlich niedriger als die Werte der anderen Gruppen (jeweils $M \geq 2.13)$. Im zweiten Fall liegt der Wert der Nichtfachkräfte mit sonstigem Ausbildungs- oder Studienabschluss $(M=3.4, S D=.52)$ deutlich höher als die Werte der anderen Gruppen (jeweils $M \leq 2.53)$. Bezüglich der Frage, wie lange man noch als Schulbegleitung arbeiten möchte, fällt auf, dass die Gruppe derer, die eine pädagogische oder pflegerische Ausbildung absolviert haben, vergleichsweise lange Zeiträume angibt ( $M \approx 10$ Jahre und 3 Monate), und die Gruppe derer, die ein pädagogisches oder pflegerisches Studium abgeschlossen haben, vergleichsweise kurze Zeiträume ( $M \approx 2$ Jahre und 11 Monate). Die Angaben derer, die über keinen Abschluss verfügen oder einen nicht einschlägigen Abschluss, liegen jeweils dazwischen. Die Zufriedenheitswerte mit bestimmten Aspekten des Arbeitsverhältnisses unterscheiden sich nicht auffällig zwischen den Teilgruppen, wobei möglich ist, dass Fachkräfte in Einzelfällen von den Trägern höher bezahlt werden als Nichtfachkräfte.

An allen Schulformen, auch an den Förderschulen, sind mehr Maßnahmen angesiedelt, die von den Jugendämtern bewilligt wurden, als die von den Sozialämtern bewilligt wurden, d. h. mehr begleitete Schüler/innen mit seelischer als mit körperlicher oder geistiger Behinderung. ${ }^{48}$ An Haupt-, Real- und Oberschulen sind sogar ausschließlich begleitete Schüler/innen mit seelischer Behinderung (für eine Übersicht siehe Abbildung 12). Das Verhältnis zwischen seelischen auf der einen und körperlichen oder geistigen Behinderungen auf der anderen Seite ist nur in Gesamtschulen annähernd ausgeglichen. Den Einschätzungen der Behinderungsart durch die Schulbegleiter/innen nach (die zwischen körperlicher und geistiger Behinderung differenzieren, allerdings bei der Gesamtzahl seelischer Behinderungen nicht mit der Gesamtzahl der Bewilligungen durch das Jugendamt übereinstimmen) werden auch an Haupt-/Real-/Oberschulen Schüler/innen mit geistiger Behinderung begleitet und an Förderschulen mehr Schüler/innen mit geistiger als mit seelischer Behinderung. Schüler/innen mit körperlicher Behinderung werden demnach nur an Grund- und Gesamtschulen begleitet.

48 Bei der Darstellung von Zusammenhängen, die zwischen verschiedenen Schulformen der weiterführenden Schulen differenzieren, bleibt das Gymnasium unberücksichtigt, da hierfür nur die Daten einer Maßnahme vorliegen. 


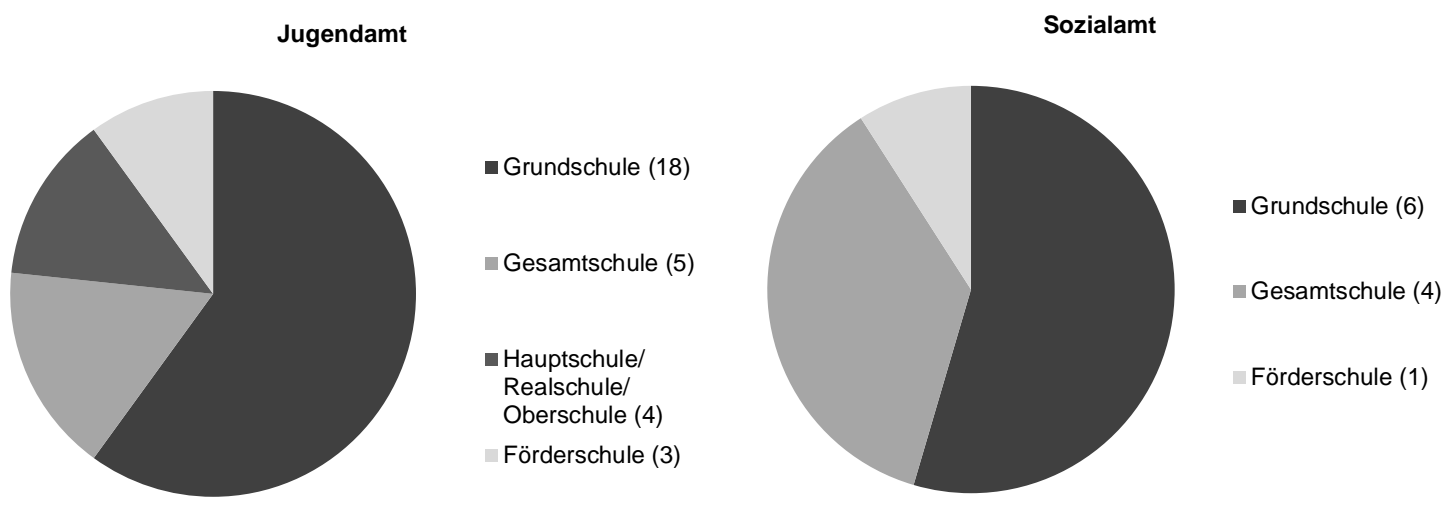

Abb. 12: Verteilung der Schulbegleitungen über die Jugendämter von Stadt und Landkreis Göttingen und über die Sozialämter von Stadt und Landkereis Göttingen auf die Schulformen (n in Klammern)

Die durchschnittliche Vertragslaufzeit (die in der Regel dem Bewilligungszeitraum entspricht) unterscheidet sich nicht nennenswert nach Art der Behinderung, nach Art des Kostenträgers (Jugendamt oder Sozialamt) oder nach der Form der besuchten Schule. Es zeigt sich lediglich, dass die befragten Schulbegleiter/innen in Jugendamtsmaßnahmen im Schnitt seit 17 Monaten für das begleitete Kind oder den begleiteten Jugendlichen zuständig sind und in Sozialamtsmaßnahmen im Schnitt seit 23 Monaten.

Zwischen der Häufigkeit von Tätigkeiten der Bereiche lebenspraktische Anforderungen, Emotions- und Verhaltensregulierung sowie didaktische Unterstützung findet sich nur hinsichtlich des Bereichs lebenspraktischer Anforderungen ein nennenswerter Unterschied zwischen den Maßnahmen, die über das Sozialamt bewilligt wurden (d. h. aufgrund geistiger oder körperlicher Behinderung; $n=10)$ und denen, die über das Jugendamt (d. h. aufgrund seelischer Behinderung; $n=30$ ) beantragt wurden. Bei Sozialamtsmaßnahmen sind Tätigkeiten in diesem Bereich häufiger $(M=3.72, S D=.8$ vs. $M=2.36, S D=.99)$. Hinsichtlich der Schulformen fällt auf, dass Tätigkeiten im Bereich lebenspraktischer Anforderungen am häufigsten an Förderschulen geleistet werden $(M=3.8, S D=1.12)$ und am seltensten an Haupt-/Real- und Oberschulen $(M=1.67, S D=0.78)$. Für Grundschulen und Gesamtschulen beträgt der Mittelwert $2.57(S D=.98)$ und $2.83(S D=1.23)$. In den Bereichen Emotions- und Verhaltensregulation sowie didaktische Unterstützung gibt es dagegen keine nennenswerten Unterschiede.

In Maßnahmen des Jugendamts Stadt liegt die Fachkraftquote mit 9 Fachkräften und 8 Nichtfachkräften höher als in Maßnahmen des Jugendamts Landkreis mit 4 Fachkräften und 10 Nichtfachkräften. Bei den Sozialämtern verhält es sich umgekehrt, allerdings sind die Fallzahlen sehr gering. Für das Sozialamt Stadt arbeiten 3 Nichtfachkräfte (und keine Fachkräfte) und für das Sozialamt Landkreis 6 Fachkräfte und 2 Nichtfachkräfte. Insgesamt arbeiten für die Jugendämter mehr Nichtfachkräfte $(n=18)$ als Fachkräfte $(n=13)$, während beide Gruppen bei den Sozialämtern etwa gleich groß sind ( $n=5$ bzw.6). Fachkräfte wie Nichtfachkräfte arbeiten an allen Schulformen, wobei in der Grundschule der Fachkraftanteil leicht überwiegt (14 Fachkräfte, 12 Nichtfachkräften) und an den weiterführenden Schulen der Anteil der Nichtfachkräfte (14 Nichtfachkräfte, 6 Fachkräfte). Tendenzielle oder signifikante Zusammenhänge zwischen didaktischen Unterstützungstätigkeiten der Schulbegleiter/innen und der Unterrichtsform zeigen sich nur für die Häufigkeit der Unterstützung bei Gruppenarbeitsphasen und der Häufigkeit von Partner- bzw. Gruppenarbeiten im Unterricht $(r=.33, p<.05$ bzw. $r=.27 p<.1)$ und zwischen der Häufigkeit der Unterstützung anderer Schüler/innen im Unterricht und der Häufigkeit von Gruppenarbeit $(r=.28, p<.1)$.

\subsection{Einbindung und Kooperation}

Im Folgenden werden zunächst die Ergebnisse zur Häufigkeit des Austauschs von Schulbegleiter/innen mit verschiedenen Personengruppen berichtet sowie ihre Einschätzungen der Wichtigkeit dieses Austauschs und der Zufriedenheit hiermit. Im Anschluss werden die Hypothesen zu der berufsbezogenen Selbstwirksamkeitserwartung, der Kooperationspraxis mit der Klassenleitung und den Kooperationswirkungen geprüft. Zuletzt werden die Ergebnisse zur Einbindung in die Schulgemeinschaft und zur Gesamtzufrieden- 
heit der Schulbegleiter/innen vorgestellt sowie Zusammenhänge zwischen kooperationsspezifischen Variablen mit weiteren Merkmalen der Maßnahme untersucht. Auch hier entspricht die Reihenfolge der Ergebnisdarstellung der Reihenfolge der Forschungsfragen.

\subsubsection{Häufigkeit und Bewertung von Austausch}

Bei den Einschätzungen der Häufigkeit des Austauschs mit verschiedenen Personengruppen zeigt sich zum einen, dass die befragten Schulbegleiter/innen im Rahmen ihrer Tätigkeit in teilweise sehr häufigem Kontakt mit verschiedenen innerschulischen oder außerschulischen Personen stehen. Zum anderen wird deutlich, dass in nicht wenigen Fällen bestimmte Kooperationspartner/innen (Koordinationskräfte, sonderpädagogische Lehrkräfte), die im Rahmen von Schulbegleitung als wichtig angenommen werden, nicht vorhanden sind. Für eine Übersicht hierzu siehe Abbildung 13.

Am häufigsten tauschen die Befragten sich mit der Klassenleitung aus. Hier geben über 90\% täglichen oder fast täglichen Austausch an und unter 5\% monatlichen oder seltener als monatlichen Austausch. Wenn andere Schulbegleiter/innen an der Schule tätig sind (was bei 3 von 45 nicht der Fall ist) und eine sonderpädagogische Lehrkraft für die Klasse zuständig ist (was bei 8 von 43 nicht der Fall ist), findet auch mit ihnen häufig Austausch statt. Gut 70\% geben täglichen oder fast täglichen Austausch und knapp 20\% wöchentlichen oder fast wöchentlichen Austausch mit anderen Schulbegleiter/innen an. Mit den zuständigen sonderpädagogischen Lehrkräften tauschen sich etwa 50\% der befragten Schulbegleiter/innen täglich oder fast täglich aus und knapp 40\% wöchentlich oder fast wöchentlich. Alle Schulbegleiter/innen geben an, sich mit den Eltern des Schülers oder der Schülerin auszutauschen, jeweils etwa ein Drittel täglich oder fast täglich und ein weiteres Drittel wöchentlich oder fast wöchentlich.

Geringer fällt die Frequenz des Austauschs (laut den Angaben der Schulbegleiter/innen, bei denen die jeweiligen Personengruppen vorhanden sind) mit weiterem Personal der Schule wie Ergotherapeut/innen oder Sozialpädagog/innen, der Schulleitung, der Koordinationskraft für alle Schulbegleiter/innen der Schule, der arbeitgebenden Einrichtung und der Koordinationskraft für den Ganztag aus. Am seltensten findet Austausch mit außerschulischen Kooperationspartner/innen wie etwa zuständigen Vertreter/innen des Jugendamts oder der Psychiatrie statt. Teilweise findet mit diesen Personengruppen gar kein Austausch statt, z. B. haben sich fast 30\% der Befragten noch nicht mit der Schulleitung ausgetauscht und knapp 50\% noch nicht mit der Koordinationskraft des Ganztags. Für die Koordinationskraft für alle Schulbegleiter/innen der Schule fallen die Angaben sehr unterschiedlich aus. Von den Befragten, an deren Schule sie vorhanden ist (weniger als die Hälfte ${ }^{49}$ ), hatten fast $24 \%$ noch keinen Austausch mit ihr, 35\% seltener als monatlich, weitere $24 \%$ monatlich, die übrigen täglich oder fast täglich. Zwei Umstände müssen bei der Bewertung der Angaben berücksichtigt werden: Zum einen, dass Austausch von den Teilnehmenden möglicherweise unterschiedlich ausgedeutet wurde, und zum anderen, dass Schulleitungen oder Koordinationskräfte ebenso wie auch sonderpädagogische Lehrkräfte gleichzeitig die zuständige Klassenleitung oder eine Fachlehrkraft sein können, wodurch sich die Austauschfrequenz erhöhen kann (eine Versuchsperson machte eine entsprechende Anmerkung). Im Fall der arbeitgebenden Einrichtung und der Kooperation mit außerschulischen Partner/innen ist zumindest diese Verzerrung ausgeschlossen. Jeweils etwas über $40 \%$ geben für den Austausch mit der Einrichtung eine Häufigkeit von monatlich oder seltener als monatlich an; knapp $10 \%$ haben sich bislang nicht ausgetauscht. Beim Austausch mit außerschulischen Partner/innen beträgt dieser Anteil über 20\% und über 60\% geben hier seltener als monatlich an. Die offene Abfrage „Sonstiger Personen“ wurde an dieser Stelle sowie bei den Fragen zur Wichtigkeit und zur Zufriedenheit hinsichtlich des Austauschs nur von wenigen Befragten genutzt, was als Hinweis darauf gewertet wird, dass alle wichtigen Personen(gruppen) mit den vorgegebenen Antwortvorgaben erfasst wurden. ${ }^{50}$ Eine Übersicht über die anteiligen Häufigkeiten des Austauschs mit zentralen schulischen Kooperationspartner/innen von Schulbegleiter/innen findet sich in Abbildung 13.

49 An dieser Stelle geben 27 Befragte an, dass keine Koordinationskraft vorhanden ist, während diese Angabe an vorheriger Stelle von 34 Befragten gemacht wird. Da anhand der Daten nicht entschieden werden kann, welche Angabe den eigentlichen Wert ausdrückt, werden beide Angaben im jeweiligen Kontext ohne Korrektur berichtet.

50 Als sonstige Personen werden darin mit einer Ausnahme Personen genannt, die vorhandenen Antwortkategorien (sonderpädagogische Lehrkraft, außerschulische Kooperationspartner/innen) zugeordnet werden können. Es wurden deshalb die Angaben dieser Personen in diesen Kategorien geprüft; alle hatten dort bereits Angaben gemacht, die für höhere Häufigkeiten des Austauschs stehen. 


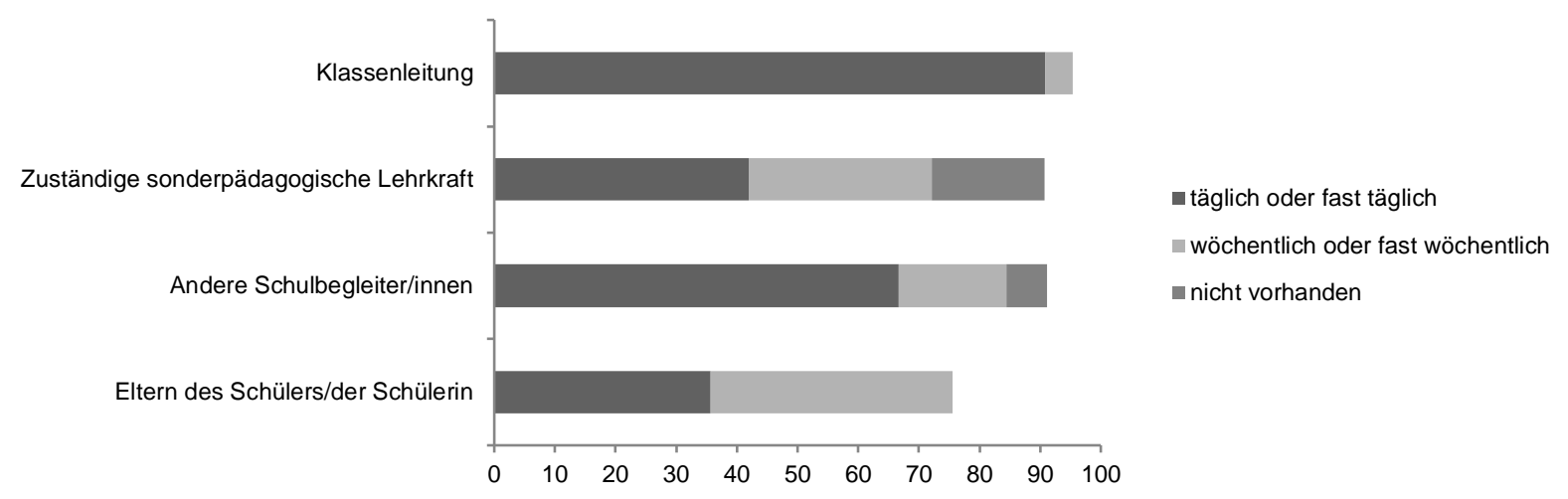

Abb. 13: Prozentuale Anteile derjenigen Schulbegleiter/innen, die täglich oder fast täglich baw. wöchentlich oder fast wöchentlich in Austausch mit den aufgeführten Personengruppen stehen bzw. bei denen die Personengruppe nicht vorbanden ist (gemessen an der Gesamtzahl der Nennungen in allen Antwortkategorien, die darüber hinaus die Kategorien monatlich, seltener als monatlich, bislang gar nicht enthielten)

Hinsichtlich der Klassenleitung sind nicht nur die Häufigkeitsangaben für Austausch besonders hoch, sondern auch die Einschätzungen der Wichtigkeit dieses Austauschs. Bis auf eine Person halten alle Befragten den Austausch mit ihr für sehr wichtig, die Kategorien eher unwichtig und sehr unwichtig wurden nicht gewählt. Auf ähnlich hohem Niveau liegen die Einschätzungen bezüglich des Austauschs mit den Eltern und der sonderpädagogischen Lehrkraft. Die hinteren Plätze belegen im Schnitt die arbeitgebende Einrichtung, die Schulleitung und die Leitung des Ganztags, die aber mit einem Mittelwert von 2.75 immer noch über dem theoretischen Skalenmittelwert - der hier eine neutrale Position bedeuten würde - von 2.5 landet. Obwohl der Austausch mit einigen Personengruppen von einem Teil der Schulbegleiterinnen für eher oder sehr unwichtig befunden wird, liegen die mittleren Wichtigkeitsbewertungen damit insgesamt auf mittlerem bis hohem Niveau (siehe Tabelle 3).

Das gleiche gilt für die Angaben zur Zufriedenheit mit dem Austausch (auch hier nur bezogen auf diejenigen, bei denen die Personengruppen vorhanden sind). Auch hier stehen die Klassenleitung und die sonderpädagogische Lehrkraft an den obersten Stellen, auf ähnlichem Niveau wie andere Schulbegleiter/innen. Am wenigsten zufrieden äußern sich die Befragten mit dem Austausch mit der Schulleitung, der Koordinationskraft für Schulbegleitung und außerschulischen Kooperationspartner/innen. Auch hier liegt der niedrigste Mittelwert mit 2.83 über dem neutralen Skalenmittelwert, so dass insgesamt von einer mittleren bis hohen Zufriedenheit gesprochen werden kann. Tabelle 3 zeigt jeweils die mittleren Werte der Wichtigkeit, die die Befragten dem Austausch mit den jeweiligen Personengruppen beimessen, und der Zufriedenheit mit diesem Austausch. 
Tab. 4: Mittelwerte der Angaben zur Bewertung des Austauschs mit verschiedenen Personengruppen

\begin{tabular}{lccc}
\hline & $\begin{array}{c}\text { Nicht } \\
\text { vorhanden }\end{array}$ & Wichtigkeit & Zufriedenheit \\
\cline { 2 - 4 } & $n$ & $M(S D)$ & $M(S D)$ \\
\cline { 2 - 4 } Klassenleitung & 1 & $3.98(.15)$ & $3.67(.80)$ \\
Zuständige sonderpädagogische Lehrkraft & 8 & $3.88(.40)$ & $3.7(.77)$ \\
Andere Schulbegleiter/innen & 3 & $3.45(.67)$ & $3.59(.55)$ \\
Eltern des Schülers oder der Schülerin & 0 & $3.91(.29)$ & $3.33(.83)$ \\
Weiteres Schulpersonal (Nicht-Lehrkräfte) & 17 & $3.67(.62)$ & $3.35(.88)$ \\
Schulleitung & 0 & $2.93(.84)$ & $3.05(1.05)$ \\
Koordinationskraft für Schulbegleitung & 27 & $3.27(.70)$ & $2.94(.77)$ \\
Außerschulische Kooperationspartner/innen & 4 & $3.34(.79)$ & $2.83(.98)$ \\
Träger (Arbeitgeber) & 0 & $3.04(.83)$ & $3.19(.83)$ \\
Leitung des Ganztags & 15 & $2.76(.93)$ & $3.44(.63)$ \\
\hline
\end{tabular}

Anmerkung. $N$ jeweils $\geq 41$.Vierstufiges Antwortformat (sehr unwichtig [unzufrieden] - eher unwichtig [unzufrieden] - eher wichtig [zufrieden] - sebr wichtig [zufrieden], Kodierung von 1 bis 4). Die Mittelwerte $(M)$ und Standardabweichungen $(S D)$ berücksichtigen nur die Angaben derjenigen, bei denen die Personengruppen vorhanden sind.

Bei vergleichender Betrachtung der Angaben zur Häufigkeit des Austauschs sowie zur Wichtigkeit und Zufriedenheit fällt auf, dass die Klassenleitung und die sonderpädagogische Lehrkraft jeweils die Spitzenpositionen einnehmen. Andere Schulbegleiter/innen gehören zwar zu den häufigsten, aber nicht zu den wichtigsten Austauschpartner/innen, und die Eltern zu den häufigsten und wichtigsten, aber nicht zu den Spitzenreitern hinsichtlich der Zufriedenheit. Direkt nach diesen Personengruppen taucht in den Angaben zur Häufigkeit und zur Wichtigkeit weiteres schulisches Personal auf, wobei durch das geschlossene Antwortformat offen bleibt, welche Kräfte im Einzelnen gemeint sind. Für die Koordinationskraft für Schulbegleitung und für die arbeitgebende Einrichtung ergeben sich in allen drei Kategorien niedrigere Werte als für die eben aufgeführten Personengruppen. Hinsichtlich der außerschulischen Kooperationspartner/innen geben die Schulbegleiter den wenigsten Austausch und die geringsten Zufriedenheitswerte an, die Einschätzung der Wichtigkeit liegt aber im Vergleich mit den anderen Personengruppen im Mittelfeld. Auch mit der Leitung des Ganztags tauschen sich Schulbegleiter/innen vergleichsweise weniger häufig aus, bewerten diesen Austausch aber auch als vergleichsweise weniger wichtig. Die Schulleitung landet bei den Häufigkeitsangaben im Mittelfeld, bei den Wichtigkeits- und Zufriedenheitsangaben auf den hinteren Plätzen.

Bei der vergleichenden Betrachtung ist (zusätzlich zur bereits angemerkten Möglichkeit von Personalunionen) die insgesamt geringe Spannbreite der Mittelwerte zu berücksichtigen; die Unterschiede in der Bewertung des Austauschs mit einzelnen Personengruppen fallen teilweise sehr gering aus. Insbesondere in den Fällen, in denen die Personengruppen bei vielen Befragten nicht vorhanden sind, schränkt die geringe Fallzahl die Aussagekraft der Werte zusätzlich ein. In diesen Fällen werden schon wenige Angaben bedeutsam, die aus Besonderheiten des Einzelfalls resultieren können und nicht den Querschnitt der Gesamtstichprobe repräsentieren.

\subsubsection{Kooperationspraxis mit der Klassenleitung und Kooperationswirkungen}

Bevor die Hypothesen zur Kooperationspraxis und zu Kooperationswirkungen geprüft werden, erfolgt eine kurze Darstellung der Deskriptivstatistik (für die Mittelwerte und Standardabweichungen der Einzelitems siehe Tabelle 4). Die Verteilungen der Mittelwerte aller Skalen (Berufsbezogene Selbstwirksamkeitserwartung, Koordination und Kokonstruktion, Emotionale Entlastung, Wertschätzung, Rollensicherheit, Arbeitszufriedenheit) sind unimodal, aber deutlich linksschief und rechtssteil, was in diesem Fall hohe Zustimmungsraten zu den jeweiligen Items bzw. hohe Merkmalausprägungen bedeutet. Bei den Skalen Emotionale 
Entlastung, Wertschätzung und Zufriedenheit fällt der Gipfel mit dem höchstmöglichen Skalenmittelwert von 4 zusammen, ebenso auch für Koordination, nicht aber für Kokonstruktion. Eine Testung auf Normalverteilung mit dem Kolmogoroff-Smirnov-Anpassungstest ergibt, dass die Verteilungen der gefundenen Skalenmittelwerte hochsignifikant von einer Normalverteilung abweichen $(p<.01)$. Eine Ausnahme bilden die Werte der Skala zu berufsbezogener Selbstwirksamkeitserwartung, die nur tendenziell von einer Normalverteilung abweichen $(\not<.1) .{ }^{51}$ Die durchschnittlichen Skalenmittelwerte liegen alle deutlich über dem theoretischen Skalenmittelwert von 2.5 und jeweils über 75\% der Skalenmittelwerte sind größer als 3. Die Antwortformate wurden dennoch überwiegend ausgeschöpft. Bis auf die Skalen Berufsbezogene Selbstwirksamkeitserwartung (niedrigster Skalenmittelwert =2.6) und Zufriedenheit (niedrigster Skalenmittelwert $=2$ ) liegen die untersten Skalenmittelwerte alle zwischen den beiden unteren Antwortkategorien 1 und 2.

Hypothese 1 lautet: Einfache Kooperation im Sinne von Koordination ist stärker ausgeprägt als komplexe Kooperation im Sinne von Kokonstruktion. Deskriptiv trifft diese Aussage bezogen auf die Werte der Stichprobe zu, der Unterschied zwischen den durchschnittlichen Skalenmittelwerten fällt aber gering aus. Für Koordination beträgt er $3.52(S D=.59, n=45)$ und für Kokonstruktion $3.4(S D=.67, n=45)$. Ein zweiseitiger $t$-Test für abhängige Stichproben ergibt, dass der Unterschied signifikant ist $(t(44)=2.34$, $p<.05)$, der Effekt fällt mit $d=0.2$ aber schwach aus. Die Nullhypothese wird abgelehnt und Hypothese 1 angenommen.

Die Hypothesen 2 und 3 sagen einen Zusammenhang zwischen der Ausprägung von Koordination bzw. Kokonstruktion und der Ausprägung von (a) emotionaler Entlastung, (b) Wertschätzung, (c) Rollensicherheit und (d) Arbeitszufriedenheit von Schulbegleiter/innen vorher. Die Hypothesenprüfung erfolgte durch Produkt-Moment-Korrelationen nach Pearson, die Ergebnisse - auch für die Gesamtskala Kooperationspraxis finden sich in Tabelle 4.

Tab. 5: Korrelationskoeffizienten für den Zusammenhang von Kooperationsformen und Kooperationswirkungen

\begin{tabular}{lcccc}
\hline & $\begin{array}{c}\text { Emotionale } \\
\text { Entlastung }\end{array}$ & Wertschätzung & Rollensicherheit & Arbeitszufriedenheit \\
\cline { 2 - 5 } Koordination & .88 & .75 & .55 & .51 \\
Kokonstruktion & .88 & .88 & .58 & .62 \\
Kooperationspraxis & .92 & .89 & .60 & .60 \\
\hline
\end{tabular}

Anmerkung. Für alle Korrelationen gilt $p<.001$ (bei jeweils $n \geq 41$ )

Die Ergebnisse zeigen höchstsignifikante und den gängigen Konventionen nach mittlere bis hohe Zusammenhänge beider Formen der Kooperation sowie der Kooperationspraxis insgesamt mit emotionaler Entlastung, wahrgenommener Wertschätzung, Rollensicherheit und Arbeitszufriedenheit der Schulbegleiter/innen. Die Hypothesen 2 und 3 werden somit angenommen. ${ }^{52}$ Hypothese 4 sagt für die Ausprägung von Kokonstruktion einen stärkeren Zusammenhang mit (a) emotionaler Entlastung, (b) wahrgenommener Wertschätzung, (c) Rollensicherheit und (d) Arbeitszufriedenheit der Schulbegleiter/innen voraus als für die Ausprägung von Koordination. Deskriptiv trifft diese Annahme hinsichtlich der Wertschätzung, der Rollensicherheit und der Arbeitszufriedenheit zu, wobei der Unterschied hinsichtlich der Arbeitszufriedenheit sehr gering ist. Die Prüfung der statistischen Signifikanz dieser Unterschiede erfolgte durch eine $z^{\prime}-$ Transformation der Korrelationswerte nach Fisher und einen anschließenden zweiseitigen ₹-Test (Lee \& Preacher, 2013). Dabei zeigte sich, dass der Korrelationskoeffizient von Kokonstruktion und Wertschätzung hochsignifikant höher liegt als der Korrelationskoeffizient von Koordination und Wertschätzung mit

51 Die Abweichung der gefundenen Skalenmittelwerte von einer Normalverteilung steht der Annahme, dass sich die Mittelwerte einer Stichprobe von mindestens $N=30$ normalverteilen (was die Anwendung parametrischer Verfahren erlaubt), nicht entgegen (siehe hierzu z. B. Bortz \& Schuster, 2010).

52 Zusätzlich gerechnete Rangkorrelationen nach Spearman, die gegenüber der Produkt-Moment-Korrelation nach Pearson weniger anfällig für eine unterschiedliche Variabilität der Werteverteilungen sind (Sedlmeier \& Renkewitz, 2013), ergeben ebenfalls signifikante Zusammenhänge vergleichbarer Stärke. 
$z=-2.99$ und $p<.01$. Die Korrelationskoeffizienten hinsichtlich der Rollensicherheit und der Arbeitszufriedenheit unterscheiden sich nicht signifikant. Entsprechend wird nur Hypothese 4b angenommen, die Ausprägung von Kokonstruktion hängt stärker mit der Ausprägung von wahrgenommener Wertschätzung zusammen als die Ausprägung von Koordination. Die Hypothesen 4a, 4c und 4d werden nicht angenommen.

Die Frage, in welchem Zusammenhang Koordination und Kokonstruktion mit der Häufigkeit des Austauschs mit der Klassenleitung stehen, wurde, da die Austauschhäufigkeit auf Ordinalskalenniveau erhoben wurde, mit Rangkorrelationen nach Spearman untersucht. Es zeigen sich signifikante mittlere Zusammenhänge der Austauschhäufigkeit mit der Ausprägung von Koordination $\left(r_{s}=.32, p<.05\right)$ und Kokonstruktion $\left(r_{s}=.37, p<.05\right)$, beide liegen auf einem ähnlichen Niveau (für die Skala Kooperationspraxis $r_{s}=.35$, $p<.05)$. Zwischen der Bewertung der Wichtigkeit der Kooperation mit der Klassenleitung und den Kooperationsskalen zeigt sich kein Zusammenhang, jedoch für die Ausprägung der Zufriedenheit, die sowohl mit Koordination $(r=.73, p<.001)$ als auch mit Kokonstruktion $(r=.73, p<.001)$ höchstsignifikant korreliert (mit der Skala Kooperationspraxis $r=.73, p<.001$ ). Hinsichtlich der Frage, in welchem Zusammenhang die berufsbezogene Selbstwirksamkeitserwartung mit der Kooperation mit der Klassenleitung und den Kooperationswirkungen steht, wurden Produkt-Moment-Korrelationen berechnet. Es ergeben sich (hoch)signifikante Zusammenhänge der berufsbezogenen Selbstwirksamkeitserwartung mit der Rollensicherheit $(r=.3$, $p<.05)$ und der Arbeitszufriedenheit $(r=.4, p<.01)$. Mit emotionaler Entlastung und der wahrgenommenen Wertschätzung zeigt sich kein Zusammenhang. Tendenziell zeigt sich ein Zusammenhang zwischen berufsbezogener Selbstwirksamkeitserwartung und Kokonstruktion $(r=.27, p<.1)$, nicht aber zwischen berufsbezogener Selbstwirksamkeitserwartung und Koordination.

Auch wenn im Rahmen dieser Arbeit keine inhaltliche Auswertung einzelner Skalenitems erfolgt, soll, um der explorativen Anlage gerecht zu werden, zuletzt noch in kurzer Form auf Auffälligkeiten bei der Bewertung einiger Einzelitems eingegangen werden. In der Skala Kokonstruktion fragt einzig das Item „Die Klassenleitung fragt (auch) bezüglich Themen, die nicht direkt meine/n Schüler/in betreffen, nach meiner Einschätzung (z. B. bezüglich anderer Schüler/innen oder der Unterrichtsgestaltung)“ explizit nach der Zusammenarbeit bei Aufgaben, die offiziell nicht im Rahmen von Schulbegleitung als Einzelfallhilfe vorgesehen sind, und es ist einzige der Kooperationsitems, dessen Mittelwert unter 3 liegt. Es ist im Vergleich also schwieriger als die anderen Items der Kooperationsskalen. In der Skala Rollensicherheit ist das Item „Mein Aufgabenbereich als Schulbegleitung ist klar abgesteckt" mit $M=2.84$ das einzige der Skala, dessen Mittelwert unter 3 fällt. Es ist im Vergleich also schwieriger als die anderen Items der Skala und als einziges Item der Skala liegt der Gipfel seiner Verteilung nicht bei dem höchsten Wert (4). Inhaltlich gesehen ist es das einzige Item, das explizit nach der Begrenzung des Aufgabenbereichs fragt. Bei der Analyse der Items zu Arbeitszufriedenheit fällt auf, dass das Item „Als Schulbegleiter/in mache ich eine wichtige Arbeit“, das von der Skalenbildung ausgeschlossen wurde, mit keinem der anderen Items (die eher auf Wohlbefinden hinsichtlich der Arbeit abzielen) korreliert. Das Empfinden von Sinnhaftigkeit oder Bedeutsamkeit scheint unabhängig zu sein von der Freude an der Arbeit.

\subsubsection{Einbindung in die Schulgemeinschaft und Gesamtzufriedenheit}

Bei den Items zur Erfassung der Einbindung in die Schulgemeinschaft wurde geprüft, ob sie zu einer Skala zusammengefasst werden können. Die Kennwerte der Skalenanalyse legen nahe, dass eine Einzelauswertung der Items aufschlussreicher ist. Das Antwortformat (trifft gar nicht $z u$ - trifft eher nicht $q u$ - trifft eher $₹ u$ - trifft voll $₹ u$, Kodierung von 1 bis 4) wurde für alle Items ausgeschöpft. Die Mittelwerte zu den Aussagen, man empfinde sich als Teil der Schulgemeinschaft bzw. man werde zu offiziellen schulischen Veranstaltungen eingeladen, liegen mit $3.42(S D=.69)$ bzw. $3.23(S D=.94)$ auf einem ähnlich hohen Niveau, wobei im ersten Fall 6.7\% und im zweiten Fall 13.6\% der Befragten der Aussage (eher) nicht zustimmen. Die Variablen korrelieren hoch $(r=.67, p<.001)$. Der Anteil der Befragten, die angeben, es treffe (eher) zu, dass sie $\mathrm{zu}$ offiziellen schulischen Veranstaltungen eingeladen werden $(79.5 \%)$ ist genauso so hoch wie der Anteil derjenigen, die dies hinsichtlich der Einladung zu Einzelfallbesprechungen/Hilfeplangesprächen/Förderplangesprächen bezüglich ihres begleiteten Schülers oder ihrer begleiteten Schülerin angeben $(M=3.41$, $S D=.92)$. Dagegen ist der Anteil geringer (53.5\%), wenn es um die Einladung zu Klassenkonferenzen, die den begleiteten Schüler oder die begleitete Schülerin betreffen, geht. Hierbei geben 25.6\% der Befragten an, diese Aussage träfe gar nicht zu, der Mittelwert liegt mit $2.58(S D=1.18)$ deutlich unter den anderen und nahe am theoretischen Skalenmittelwert, der eine neutrale Position bedeutet. 
Die Werte für die Gesamtzufriedenheit fallen positiv aus: Über die Hälfte (57.8\%) der Befragten beantwortet die Frage danach, ob sie insgesamt mit der Einbindung und Zusammenarbeit in der Schule zufrieden sind, mit ja und ein knappes Drittel (31.1\%) mit eher ja und nur ein gutes Zehntel (11.1\%) mit nein oder eher nein. Als häufigster Grund für die Zufriedenheit wurden von denjenigen, die zuvor mit ja oder eher ja geantwortet hatten $(n=40)$, die Wertschätzung ihrer Arbeit angegeben $(n=34)$, gefolgt von der Sympathie zwischen der Klassenleitung und ihnen $(n=33)$, regelmäßigen Besprechungen $(n=29)$ und Feedback zu ihrer Arbeit $(n=23)$. Eine genaue Anleitung ist nur für 9 Befragte ein wichtiger Grund für ihre Zufriedenheit und eine feste Ansprechperson für Schulbegleitung an ihrer Schule für 4 Befragte..$^{33}$ Die Möglichkeit der eigenen Eintragung von wichtigen Gründen nutzten 8 der Befragten, teilweise durch längere Ausführungen. Dabei berichteten 4 von einer guten Atmosphäre/Stimmung bei der Arbeit, 3 von Akzeptanz/Wertschätzung durch verschiedene Personengruppen in der Schule und 2 von einer guten Einbindung in den Schulalltag. Alle, die auf die Frage nach Zufriedenheit mit (eher) nein geantwortet hatten $(n=5)$, gaben als wichtigen Grund „keine regelmäßigen Besprechungen“ an. Die nächstkleinere Zahl an Nennungen fiel auf „mangelnde Wertschätzung meiner Arbeit" $(n=4)$ und „mangelnde Zeit seitens der Klassenleitung“ $(n=3)$. Jeweils zweimal wurden außerdem „mangelnde Sympathie zwischen der Klassenleitung und mir" und „keine feste Ansprechperson“ genannt, jeweils einmal „,mangelnde Anleitung“, „zu wenig Feedback“ und „sonstiges“. „Mangelnde Zeit von meiner Seite aus“ wurde von keinem als wichtiger Grund ausgewählt.

\subsubsection{Zusammenhänge von Kooperation mit weiteren Merkmalen}

Die Untersuchung von Zusammenhängen der Kooperation mit der Klassenleitung mit der Qualifikation der Schulbegleiter/innen, ihrer bisherigen Tätigkeitsdauer, der Schulform und den von ihnen ausgeführten Tätigkeiten erfolgt anhand des Vergleichs von Fallzahlen (Kreuztabellen) und Gruppenmittelwerten. Aufgrund teilweise sehr geringer Fallzahlen bzw. Gruppengrößen konnten für einige Fragen keine inferenzstatistischen Analysen vorgenommen werden, so dass die Ergebnisdarstellung an den Stellen auf rein deskriptivem Niveau erfolgt.

Es zeigen sich deskriptiv keine nennenswerten Unterschiede in der Ausprägung der Kooperation (Koordination, Kokonstruktion und Kooperationspraxis insgesamt) zwischen den Fachkräften, den Nichtfachkräften mit abgeschlossenem Studium oder Ausbildung, den in Ausbildung oder Studium befindlichen Nichtfachkräften und der Gruppe derer, die keine Ausbildung und kein Studium abgeschlossen hat und auch nicht darin befindlich ist.

Korrelationsanalysen zwischen der bisherigen Dauer der Tätigkeit als Schulbegleitung und der Ausprägung von Koordination, Kokonstruktion und der Kooperationspraxis insgesamt zeigen keine Zusammenhänge. Zwischen den Schulformen zeigen sich deskriptiv insgesamt kaum Unterschiede zwischen der Ausprägung von Kooperation, lediglich die Kooperationswerte an Haupt-, Real- und Oberschulen liegen für Koordination $(M=3.1, S D=.88)$ leicht und für Kokonstruktion $(M=2.61, S D=.84)$ deutlich niedriger als an anderen Schulen (jeweils $M \geq 3.38$ für Koordination und $M \geq 3.48$ für Kokonstruktion). Zwischen Jugendamts- und Sozialamtsmaßnahmen finden sich deskriptiv kaum Unterschiede in der Ausprägung der Kooperation, ebenso wenig, wenn nach Art der Behinderung des begleiteten Schülers oder der begleiteten Schülerin differenziert wird. Korrelationsanalysen zeigen keine Zusammenhänge zwischen der Ausprägung von Kooperation mit der Klassenleitung und der Häufigkeit von didaktischer Unterstützung des Schülers oder der Schülerin durch die Schulbegleitung und der Unterstützung bei der Emotions- und Verhaltensregulation. Die Häufigkeit der Unterstützung bei lebenspraktischen Anforderungen korreliert signifikant positiv auf mittlerem Niveau mit der Ausprägung von Kokonstruktion $(r=.37, p<.05)$, nicht aber mit der Ausprägung von Koordination (Korrelationswerte mit der Gesamtskala Kooperationspraxis $r=.37$, $p<.05)$.

Zwischen der Kooperation mit der Klassenleitung und der Einbindung in die Schulgemeinschaft zeigen sich verschiedene Zusammenhänge. Die Ausprägung von Koordination und Kokonstruktion (und der Kooperationspraxis insgesamt) korreliert jeweils höchstsignifikant mit der Empfindung, Teil der Schulgemeinschaft zu sein, sowie damit, dass man zu offiziellen schulischen Veranstaltungen eingeladen wird (jeweils

53 Es sollten die wichtigsten drei Gründe ausgewählt werden, womit verhindert werden sollte, dass ohne Differenzierung der Wichtigkeit alle Gründe angekreuzt werden. Die Gesamtsumme der Nennungen lässt darauf schließen, dass im Schnitt mehr als drei Gründe ausgewählt wurden. Zwar führt dies evtl. zu einer Überrepräsentation der Angaben von denjenigen Versuchspersonen, die die Begrenzung überschritten haben. Eine nachträgliche Korrektur ist aber nicht möglich und eine Sichtung der Daten zeigt, dass nicht nur einzelne, sondern ein großer Teil der Versuchspersonen mehr als drei Gründe angegeben hat. Es werden von daher alle Angaben gleichbehandelt (dasselbe gilt für die Angaben zu Gründen für Unzufriedenheit). 
$r>.65, p<.001)$. Die Einladung zu Klassenkonferenzen hängt lediglich mit der Ausprägung von Kokonstruktion zusammen $(r=.31, p<.05)$, nicht aber mit der Ausprägung von Koordination oder der Kooperationspraxis insgesamt. Hinsichtlich der Einladung zu Hilfeplan-/Förderplan-/Einzelfallbesprechungen zeigen sich nur tendenzielle Zusammenhänge zu Koordination $(r=.25, p<.1)$ und Kokonstruktion $(r=.26, p<.1$; Werte für die Kooperationspraxis insgesamt $r=.28, p<.1)$.

Zum Zusammenhang von Schulform und der Einbindung in die Schulgemeinschaft fällt auf, dass die Werte für alle vier Items (die Empfindung als Teil der Schulgemeinschaft, die Einladung zu offiziellen schulischen Veranstaltung, die Einladung zu Klassenkonferenzen, die Einladung zu Hilfeplan-/Förderplan/Einzelfallbesprechungen) bei denjenigen Schulbegleiter/innen, die an Haupt-, Real- und Oberschulen tätig sind $(n=5$ ), am niedrigsten ausfallen (der von den vier Items höchste Mittelwert beträgt 2.6, $S D=.89$ ) und bei denjenigen, die an Förderschulen tätig sind $(n=5)$, am höchsten (der von den vier Items niedrigste Mittelwert beträgt 3.6, $S D=.55)$.

\subsubsection{Offene Fragen}

Bei der offenen Abfrage nach Verbesserungsvorschlägen zur Zusammenarbeit an der Schule machten 12 Befragte teilweise ausführliche Angaben. Dabei werden am häufigsten $(n=6)$ der Wunsch nach regelmäßigen Besprechungen, nach einer Koordinationskraft an der Schule $(n=3)$ und nach einer generell besseren Kommunikation zwischen den Beteiligten $(n=3)$ geäußert. Dabei werden teilweise neben der Klassenleitung und der sonderpädagogischen Lehrkraft auch die Fachlehrkräfte, die Eltern und die bewilligenden Ämter genannt. Einzelne Vorschläge beziehen sich auf die Erarbeitung eines schulischen Konzepts für Inklusion, die Teilnahme an Besprechungen der Klassenleitung und der sonderpädagogischen Lehrkraft, mehr Zeit für Kooperation und einem Aufenthaltsraum für Schulbegleiter/innen (die aus Platzgründen die Lehrerzimmer nicht nutzen können).

Allgemeine Angaben zur Umfrage machen 13 Befragte. Bis auf zwei kurze Angaben, die sich direkt auf den Fragebogen beziehen, werden (wiederum teilweise ausführliche) Anmerkungen zur Maßnahme Schulbegleitungen gemacht. Von sechs Befragten werden die Arbeitsbedingungen (kurze Vertragslaufzeiten, keine Vergütung im Krankheitsfall des Schülers oder der Schülerin, geringer Lohn) kritisiert. Thematisiert wird auch die Notwendigkeit einer Qualifikation von Schulbegleiter/innen $(n=4)$ und die Unstimmigkeit des Konzepts von Schulbegleitung $(n=2)$. Einzelne Befragte merken an, dass Schulbegleiter/innen in der Praxis mehr Aufgaben übernehmen als offiziell vorgesehen, da die Abgrenzung zu schulisch-pädagogischen Aufgaben nicht realistisch sei. So schreibt eine Person, dass „eine nichtpädagogisch arbeitende Schulbegleitung völliger Schwachsinn“ sei, eine andere schlägt vor, „die Aufgabe als Co-Lehrer zu definieren mit Schwerpunkt begleiteter Schüler". In diesem Zusammenhang werden von einer Person auch Bedenken geäußert, dass Einzelbegleitung zu Unselbstständigkeit und Abhängigkeit des begleiteten Kindes oder Jugendlichen führen kann. 


\section{Diskussion und Ausblick}

Ziel der Studie war zum einen, einen Überblick über zentralen Daten der Maßnahme Schulbegleitung in Stadt und Landkreis Göttingen zu ermöglichen. Zum anderen sollten Erkenntnisse über die Einbindung von Schulbegleiter/innen an ihrer Einsatzschule gewonnen werden, insbesondere hinsichtlich der Kooperation mit der Klassenleitung und möglichen Zusammenhängen dieser Kooperation mit arbeitsbezogenen Merkmalen der Schulbegleiter/innen. Im Folgenden werden die Ergebnisse der Untersuchung inhaltlich interpretiert und diskutiert, welche Implikationen sich für weitere Forschung und für die schulische Praxis ergeben. Hierzu werden die Ergebnisse zunächst zusammengefasst und auf ihre Aussagekraft geprüft, bevor im zweiten Schritt eine Einordnung in den bisherigen Forschungsstand vorgenommen wird. Anschließend werden Schlussfolgerungen für Theorie und Praxis diskutiert, bevor ein abschließendes Fazit gezogen wird.

\subsection{Zusammenfassung der Ergebnisse}

Zur Beschreibung der Schulbegleiterschaft in Stadt und Landkreis Göttingen lässt sich feststellen, dass sie eine hohe Altersheterogenität und eine hohe Geschlechtshomogenität mit einem deutlichen höheren Frauenanteil (87\%) aufweist. Alle Schulbegleiter/innen besitzen einen Schulabschluss (die Mehrheit Abitur oder vergleichbare Abschlüsse). Bezüglich des Studien- und Ausbildungshintergrunds zeigt sich ein heterogenes Bild. Knapp 70\% haben eine Ausbildung oder ein Studium abgeschlossen und 13\% befinden sich in Ausbildung oder Studium. Eine einschlägige formale Qualifikation (d. h. im pädagogischen oder pflegerischen Bereich) besitzen etwa 45\% der Befragten, etwa zu gleichem Anteil in Form eines Studien- bzw. eines Ausbildungsabschlusses. Auffällig ist, dass 13\% über mehr als einen Berufsabschluss verfügen oder sich nach einem ersten Abschluss erneut in Ausbildung oder Studium befinden. Der Anteil derjenigen, die keinen formalen Berufsabschluss besitzt und sich auch nicht in Ausbildung oder Studium befindet, beträgt 20\%. Der Anteil derer, die vor der Tätigkeit als Schulbegleitung bereits beruflich mit Kindern oder Jugendlichen gearbeitet haben, liegt mit 70\% deutlich über der Fachkraftquote. Die Hälfte der Befragten hat bereits ehrenamtlich mit Kindern oder Jugendlichen gearbeitet. Die Mehrheit der Befragten ist bislang weniger als 3 Jahre als Schulbegleitung tätig und etwa ein Viertel länger als 4 Jahre. Die Angaben zur gewünschten weiteren Tätigkeitsdauer variieren sehr stark. Knapp 30\% wollen noch mehr als 8 Jahre als Schulbegleitung arbeiten, knapp 20\% weniger als einen Monat. Bei den Gründen für die Aufnahme der Schulbegleitertätigkeit überwiegen deutlich die Gründe, die sich auf die Arbeitsinhalte beziehen, gegenüber denen, die als Nützlichkeitsgründe bezeichnet werden können. Bei einem nicht geringen Teil der Befragten spielen aber auch diese Gründe eine Rolle, z. B. dient die Tätigkeit in etwa der Hälfte der Fälle (auch) der beruflichen Neuorientierung und stellt für etwa ein Drittel eine Verlegenheitslösung dar, weil im eigentlich gewünschten Tätigkeitsfeld keine Arbeit gefunden wurde. 
Die Befragten arbeiten zum großen Teil auf Angestelltenbasis (77\%) und zu einem kleineren Teil auf Honorarbasis $(23 \%)$ bei einem freien Träger. Die meisten Verträge sind über ein Jahr ausgestellt, nur wenige über einen längeren Zeitraum. Mit den Vertragsbedingungen zeigen sich die Befragten unterschiedlich zufrieden, wobei die Urlaubsregelungen und die Entlohnung besser bewertet werden als die Vertragslaufzeit und die Regelungen im Krankheitsfall. In der Mehrzahl der Fälle (knapp 80\%) bietet der Träger Schulungen an, etwa $60 \%$ der Befragten haben bereits an einer teilgenommen. Bis auf zwei Personen sind alle Befragten nur für einen Schüler oder eine Schülerin zuständig, im Schnitt seit eineinhalb Jahren. Nur ein geringer Anteil von 9\% arbeitet bereits mehr als 3 Jahre mit dem Schüler oder der Schülerin. Deutlich mehr Maßnahmen (insgesamt 74\%) werden über die Jugendämter als über die Sozialämter finanziert, was einem deutlichen höheren Anteil von seelischer Behinderung als Beantragungsgrund gegenüber geistiger oder körperlicher Behinderung entspricht. Den Schätzungen der Schulbegleiter/innen nach liegt der Anteil seelischer Behinderungen nicht ganz so hoch und der geistiger Behinderungen etwas höher. Über die Hälfte der Schulbegleitungen (57\%) wird an Grundschulen geleistet. Gesamtschulen kommen auf einen Anteil von fast 20\%, weiterführende Schulen (ohne Gymnasien) und Förderschulen auf jeweils knapp 10\%. Im Schnitt beträgt der Maßnahmenumfang wöchentlich 8 Stunden bei Angaben zwischen 4 Stunden und 40 Stunden. Im begleiteten Unterricht wird mit verschiedenen Lehr- und Lernformen gearbeitet, wobei den Angaben der Befragten nach insgesamt häufiger Einzelarbeit als Gruppenarbeit eingesetzt wird. In 26\% der Fälle ist eine weitere Schulbegleitung in der gleichen Klasse tätig.

Hinsichtlich des Tätigkeitsprofils von Schulbegleiter/innen zeigt sich, dass die Unterstützung bei der Emotions- und Verhaltensregulation weiten Raum einnimmt. So geben z. B. 70\% der Befragten an, dass Intervention bei (auto)aggressivem Verhalten eine Tätigkeit ist, die sie oft oder sehr oft ausführen. Auch didaktische Unterstützung im Unterricht wird von der Mehrheit der Schulbegleiter/innen (83\%) oft oder sehr oft geleistet, allerdings bezieht sich die Unterstützung bei etwa der Hälfte der Befragten vorrangig auf das begleitete Kind oder die/den begleitete/n Jugendliche/n. Die Unterstützung im Bereich lebenspraktischer Anforderungen nimmt gegenüber den anderen Aufgabenbereichen weniger Raum ein. So gehören z. B. Tätigkeiten in der Pflege und Mobilitätshilfe bei etwa 75\% der Befragten überhaupt nicht zu ihrem Aufgabenbereich. Zum Zusammenhang der Aufgabenbereiche zeigen die Ergebnisse, dass die Häufigkeit der didaktischen Unterstützung mit der Unterstützung bei der Emotions- und Verhaltensregulation und der Unterstützung bei lebenspraktischen Anforderungen zusammenhängt. Zwischen den Tätigkeiten der beiden Bereiche Emotions- und Verhaltensregulation und der Unterstützung bei lebenspraktischen Anforderungen zeigen sich nur vereinzelte Zusammenhänge. Auf deskriptivem Niveau finden sich Zusammenhänge von der Art der Behinderung bzw. dem bewilligenden Amt mit verschiedenen Aspekten der Maßnahme Schulbegleitung. An allen Schulformen sind mehr Jugendamtsmaßnahmen als Sozialamtsmaßnahmen angesiedelt. Die befragten Schulbegleiter/innen, die an Haupt-, Real- und Oberschule tätig sind, arbeiten in Jugendamtsmaßnahmen. Schulbegleiter/innen, die über das Sozialamt finanziert werden, sind im Schnitt bereits länger für den aktuell begleiteten Schüler oder die aktuell begleitete Schülerin tätig und übernehmen häufiger Aufgaben im Bereich lebenspraktischer Anforderungen als Schulbegleiter/innen, die über das Jugendamt finanziert werden. Dieser Aufgabenbereich nimmt an Förderschulen mehr Raum ein als an den anderen Schulformen und spielt insbesondere in Haupt-, Real- und Oberschulen keine nennenswerte Rolle. Die Fachkraftquote liegt in Grundschulen höher als in weiterführenden Schulen. Hinsichtlich des Tätigkeitsprofils von Schulbegleiter/innen zeigt sich, dass, wenn häufiger Gruppenarbeit stattfindet, häufiger auch andere Schüler/innen im Unterricht unterstützt werden.

Die Ergebnisse zum Austausch mit verschiedenen Personengruppen zeigen, dass die Klassenleitung diejenige Person ist, mit der sich die Schulbegleiter/innen mit Abstand am häufigsten austauschen (über $90 \%$ täglich oder fast täglich) und mit der sie Austausch für am wichtigsten halten. Auch die sonderpädagogischen Lehrkräfte, andere Schulbegleiter/innen und die Eltern des Kindes oder Jugendlichen sind wichtige Austauschpartner/innen, wobei bei fast 20\% der Befragten keine sonderpädagogische Lehrkraft für die Klasse zuständig ist. Weniger Austausch findet mit weiterem schulischen Personal, der Koordinationskraft für Schulbegleitungen und dem Maßnahmenträger statt. Kaum Austausch besteht mit der Leitung des Ganztags und außerschulischen Kooperationspartner/innen. Insgesamt zeigt sich, dass den befragten Schulbegleiter/innen der Austausch mit verschiedenen Personengruppen wichtig ist (insbesondere mit der Klassenleitung, der sonderpädagogischen Lehrkraft und den Eltern des Kindes oder Jugendlichen) und sie weitgehend zufrieden mit dem Austausch mit verschiedenen Personengruppen sind (insbesondere mit dem Austausch mit der Klassenleitung, der sonderpädagogischen Lehrkraft und den anderen Schulbegleiter/innen). 
Die Werte zur Kooperationspraxis mit der Klassenleitung zeigen, dass insgesamt von einer ausgeprägten Kooperation zwischen Klassenleitung und Schulbegleitung ausgegangen werden kann, wobei Kooperation in einfacher Form (Koordination) gegenüber anspruchsvollerer Form (Kokonstruktion) überwiegt. Es bestehen Zusammenhänge zwischen beiden Kooperationsformen mit der emotionalen Entlastung, die Schulbegleiter/innen durch die Kooperation empfinden, und der wahrgenommenen Wertschätzung durch die Klassenleitung. Dabei fällt die wahrgenommene Wertschätzung höher aus, wenn Kooperation in anspruchsvollerer Form (Kokonstruktion) stattfindet. Weiterhin zeigt sich, dass die Ausprägung von Kooperation (in beiden Formen) mit der Ausprägung von Sicherheit über die eigene Rolle als Schulbegleitung und mit der Zufriedenheit mit der Tätigkeit als Schulbegleitung zusammenhängt. Insgesamt fallen die Werte für emotionale Entlastung, wahrgenommene Wertschätzung, Rollensicherheit und Arbeitszufriedenheit positiv aus. Die Ausprägung der beiden Kooperationsformen Koordination und Kokonstruktion hängt positiv mit der Häufigkeit des Austauschs mit der Klassenleitung und der Zufriedenheit hiermit zusammen. Die berufsbezogene Selbstwirksamkeitserwartung der Schulbegleiter/innen zeigt keinen Zusammenhang mit Kooperation, aber mit der Rollensicherheit und der Arbeitszufriedenheit der Schulbegleiter/innen. Die große Mehrheit der Schulbegleiter/innen fühlt sich als Teil der Schulgemeinschaft und wird zu offiziellen schulischen Veranstaltungen und zu Besprechungen bezüglich des begleiteten Schülers oder der begleiteten Schülerin eingeladen (z. B. zu Förder- oder Hilfeplangesprächen), wohingegen nur etwa die Hälfte zu Klassenkonferenzen eingeladen wird. Insgesamt zeigen sich fast $90 \%$ der Befragten zufrieden mit der Einbindung und Zusammenarbeit an ihrer Schule. Als wichtige Faktoren hierfür wird die Wertschätzung ihrer Arbeit und Sympathie zwischen ihnen und der Klassenleitung angegeben. Auch regelmäßige Besprechungen und Feedback spielen eine wichtige Rolle, eine genaue Anleitung der Tätigkeit dagegen weniger.

In der Zusammenschau der erfassten Daten fällt auf, dass sowohl die Ausprägung der Kooperation mit der Klassenleitung als auch die Angaben zur Einbindung in die Schulgemeinschaft für Haupt-, Real- und Oberschulen ungünstiger ausfallen als für die anderen Schulformen. Die stärkste Einbindung in die Schulgemeinschaft wird für Förderschulen berichtet. Die Kooperation mit der Klassenleitung hängt positiv mit Angaben zur Einbindung in die Schulgemeinschaft zusammen. Von 12 Befragten wird die Möglichkeit genutzt, offene Angaben zur Verbesserung der schulischen Zusammenarbeit zu machen. Dabei werden vor allem regelmäßige Besprechungen gewünscht. Zudem finden sich in den offenen Angaben viele kritische Anmerkungen zur Maßnahme Schulbegleitung. Kritisiert werden die Arbeitsbedingungen und die unstimmige Konzeption der Maßnahme.

\subsection{Aussagekraft der Ergebnisse}

Die inhaltliche Aussagekraft der Ergebnisse kann auf zwei Ebenen diskutiert werden: der Ebene der Stichprobe und der Ebene des Erhebungsinstruments. Die Befragung war als Vollerhebung angelegt, d. h. alle Schulbegleiter/innen in Stadt und Landkreis Göttingen ${ }^{54}$ wurden per Mail oder Post kontaktiert und um ihre Teilnahme gebeten. Der Bitte kamen 47 Schulbegleiter/innen nach, was einer Rücklaufquote von einem knappen Drittel entspricht. Die Ausschöpfungsquote liegt im Rahmen dessen, was bei vergleichbaren Studien üblich ist (vgl. Kißgen et al., 2013), und erlaubt mit Einschränkungen eine Interpretation der Ergebnisse in Bezug auf die Grundgesamtheit. Einschränkungen der Übertragbarkeit ergeben sich zum einen daraus, dass die Stichprobe unabhängig von ihrem Anteil an der Grundgesamtheit relativ klein ist, sodass mögliche Besonderheiten von Einzelfällen stärker verzerrend auf die Gesamtwerte der Stichprobe wirken können als bei einer höheren Fallzahl. Zum anderen ist die Stichprobe möglicherweise selektiv und stellt keinen gleichmäßigen Ausschnitt der Population der Schulbegleiter/innen dar. Tendenziell sind sozial privilegierte, überdurchschnittlich intelligente und weibliche Personen in Stichproben freiwilliger Studien überrepräsentiert (Bortz \& Döring, 2006). Durch das Internet als Befragungsmedium ist zudem denkbar, dass eher jüngere Menschen an der Umfrage teilgenommen haben (vgl. ebd.; Zierer et al., 2013). Wie sich die Stichprobe der vorliegenden Studie im Vergleich zur Grundgesamtheit im Einzelnen zusammensetzt, ist nicht festzustellen, da über die Grundgesamtheit keine Daten vorliegen. Lediglich ein Abgleich mit der Verteilung der Fallzahlen auf die Ämter zum Jahresende ist möglich. Geht man davon aus, dass die Fallzahlen der Ämter zum Zeitraum der Umfrage (Juli) in einem ähnlichen Verhältnis zueinanderstanden, bildet die Stichprobe in etwa den tatsächlichen Anteil an Maßnahmen nach Jugendhilfe (Stichprobe 73.8\%, Fallzahlen 66\%) bzw. nach

54 Einschränkend gilt, wie eingangs erläutert, dass möglicherweise noch vereinzelte Maßnahmen im Rahmen des Eltern-Arbeitgeber-Modells durchgeführt werden; diese wurden nicht erfasst. 
Sozialhilfe (Stichprobe 26\%, Fallzahlen 34\%) ab, wobei Maßnahmen über das Jugendamt Stadt (Stichprobe 40.5\%, Fallzahlen 21\%) stark überrepräsentiert sind gegenüber Maßnahmen des Jugendamts Landkreis (Stichprobe 33.3\%, Fallzahlen 45\%). Darüber hinaus liefern die Daten der Untersuchung keine Hinweise auf eine Verzerrung der Stichprobe. ${ }^{55}$

Einschränkungen der Interpretierbarkeit der Ergebnisse, die aus dem Erhebungsinstrument resultieren, können hinsichtlich der deskriptiven Daten zur Maßnahme und hinsichtlich der Einschätzung von Kooperation und Kooperationswirkungen diskutiert werden. Die Möglichkeit, am Ende der Umfrage wichtige Fragen zu ergänzen, die den Befragten aus eigener Einschätzung gefehlt haben, wurde von keinem der Befragten wahrgenommen. Auch die Ergänzung eigener Antwortmöglichkeiten zu den Fragen, die auf die Beschreibung der Maßnahme Schulbegleitung abzielen, wurde eher wenig in Anspruch genommen. Lediglich die Ergänzungen, die ein Teil der Befragten hinsichtlich der von ihnen ausgeführten Tätigkeiten macht, liefern den Hinweis, dass unterrichtsbezogene Tätigkeiten differenzierter hätten erfasst werden können. Insgesamt lässt sich davon ausgehen, dass der zu erfassende Untersuchungsbereich mit den gestellten Fragen weitgehend abgedeckt werden konnte. Schwieriger einzuschätzen ist die Güte der Messung der Kooperationspraxis und der angenommenen Kooperationswirkungen. Einschränkungen der Güte können sich aus einem Mangel an Objektivität, Reliabilität und Validität ergeben (vgl. Zierer et al., 2013). Zur Wahrung gleicher Durchführungsvoraussetzungen wurden genaue Instruktionen in den Fragebogen eingefügt, zur Wahrung der Auswertungs- und Interpretationsobjektivität wurden in dieser Arbeit die einzelnen Analyseschritte ausführlich dargelegt. Die Reliabilität der Skalen liegt mit $a \geq .7$ durchgängig auf akzeptablem bis hohen Niveau, was insbesondere angesichts der teilweise geringen Itemzahl pro Skala positiv zu bewerten ist (Bortz \& Döring, 2006; Bühner, 2011). Dabei muss allerdings beachtet werden, dass hohe Homogenität von Skalen auch ein Ausdruck ihrer Inhaltsarmut sein kann (Bühner, 2011). Insbesondere die Reliabilitätswerte der Skalen Emotionale Entlastung und Wertschätzung fallen hoch aus (jeweils $a>$.9). Um die Konstrukt- und die Inhaltsvalidität der Messung zu sichern, sollte in Folgestudien geprüft werden, ob die Skalenitems die Konstrukte wirklich in ihrer Breite abdecken (Kuckartz et al., 2013). Einer Überprüfung sollten auch die Skalen zur Kooperationspraxis unterzogen werden: Zwar zeigen sich differenzierte Zusammenhänge beider Skalen mit anderen Merkmalen und ein hypothesenkonformer (wenn auch geringer) Unterschied zwischen den Skalenmittelwerten, was darauf hinweist, dass sie tatsächlich unterscheidbare Formen von Kooperation abbilden. Die Betrachtung bivariater Korrelationen auf Itemebene ermöglichte aber keine klare Trennung von zwei Dimensionen von Kooperation, so dass eine faktorenanalytische Skalenüberprüfung anhand einer größeren Stichprobe sinnvoll wäre. Zur Angemessenheit der neu entwickelten Skalen muss darüber hinaus angemerkt werden, dass die insgesamt eher hohen Werte einen Deckeneffekt nahelegen, der auf eine geringe Itemschwierigkeit hinweist (Bortz \& Döring, 2006). Zur Messung von latenten Konstrukten ist eine geringe Itemschwierigkeit eher ungünstig, da Unterschiede in der wahren Merkmalsausprägung (in diesem Fall insbesondere zwischen Personen mit einer eher hohen Merkmalsausprägung) auf diese Weise nicht differenziert erfasst werden können. Da für die neu entwickelten Items keine Vergleichswerte anderer Stichproben vorliegen, kann nicht beurteilt werden, inwieweit der hohe Zustimmungsgrad Ausdruck einer zu geringen Itemschwierigkeit oder Ausdruck einer hohen Merkmalsausprägung ist. Hinsichtlich der Skalen Selbstwirksamkeitserwartung und Arbeitszufriedenheit ist dagegen ein eingeschränkter Vergleich mit anderen Stichproben möglich, da diese Skalen mit nur geringen Adaptionen von Schwarzer und Schmitz (1999) und Bos et al. (2010) übernommen wurden. Die durchschnittlichen Itemund Skalenmittelwerte der befragten Schulbegleiter/innen liegen jeweils wesentlich höher als die der in den jeweiligen Studien befragten Lehrkräfte. Der Skalenmittelwert zur berufsbezogenen Selbstwirksamkeitserwartung liegt in der Stichprobe von Schwarzer und Schmitz (1999) zwischen den Antwortkategorien stimmt kaum und stimmt eher, der Skalenmittelwert zur Berufszufriedenheit in der Stichprobe von Bos et al. (2010) zwischen den Mittelwerten stimme wenig zu und stimme überhaupt nicht zu. Die Skalenmittelwerte der Schulbegleiterstichprobe liegen demgegenüber in beiden Fällen zwischen den Antwortkategorien trifft eher ₹u und trifft voll $\approx u$. Die berufsbezogene Selbstwirksamkeitserwartung und Arbeitszufriedenheit sind in der Stichprobe also vergleichsweise hoch ausgeprägt.

55 Die Anzahl und Länge sowie der Inhalt der offenen Anmerkungen zur Studie könnte vermuten lassen, dass bei einem Teil der Befragten das Bedürfnis, auf Verbesserungsbedarf zu Schulbegleitung hinzuweisen, zur Studienteilnahme motiviert hat. Da die Zufriedenheitswerte aber insgesamt hoch ausfallen, ist nicht davon auszugehen, dass die Stichprobe sich vorrangig aus den eher weniger zufriedenen Schulbegleiter/innen zusammensetzt. 
Trotz der genannten Einschränkungen kann festgehalten werden, dass das eingesetzte Erhebungsinstrument sich bei der Hypothesenüberprüfung bewährt hat, was für die grundsätzliche Brauchbarkeit der entwickelten Skalen spricht.

\subsection{Einordnung der Ergebnisse}

Im Folgenden werden die Ergebnisse der Untersuchung zum einen in den Forschungsstand zu Schulbegleitung und zum anderen in den Forschungsstand zu Kooperation an Schulen eingeordnet.

Die Daten zur Beschreibung der Schulbegleiterschaft und zur Maßnahme Schulbegleitung entsprechen in weiten Teilen denen bisheriger Studien, in denen vergleichbare Daten erhoben wurden. Die Schulbegleiter/innen in Stadt und Landkreis Göttingen weisen ebenso wie die Schulbegleiter/innen anderer Stichproben (Henn et al., 2014; Wohlgemuth, 2009) einen hohen Frauenanteil und eine breite Altersspanne auf. Auch die Fachkraftquote liegt mit etwa 45\% auf einem ähnlichen Niveau wie in anderen Regionen (Beck et al., 2010; Dworschak 2012b; Henn et al., 2014; Wohlgemuth, 2009), ebenso wie die bisherige Tätigkeitsdauer als Schulbegleitung (Dworschak, 2012b, 2012c). Dass sowohl im Göttinger Raum als auch anderswo die meisten Schulbegleiter/innen erst seit wenigen Jahren tätig sind, kann auf eine hohe Fluktuation hindeuten. Hierbei ist zu bedenken, dass der rasante Anstieg von Schulbegleitungen in den letzten Jahren notwendigerweise viele Neueinstellungen mit sich gebracht hat. Die hohe Varianz der gewünschten weiteren Tätigkeitsdauer, die die Schulbegleiter/innen der vorliegenden Stichprobe angeben, deutet aber daraufhin, dass Schulbegleitung zumindest für einen Teil der Beschäftigten keine langfristige Perspektive ist. Dies spiegelt sich auch in den Angaben der Gründe zur Aufnahme der Tätigkeit wieder. Für viele Schulbegleiter/innen bietet die Tätigkeit die Chance einer beruflichen Neuorientierung und für einige ist sie eine Verlegenheitslösung. Neben Erkenntnissen zur Motivation von Schulbegleiter/innen ergänzt die vorliegende Untersuchung bisherige Befunde auch um Erkenntnisse über die Erfahrungen, die Schulbegleiter/innen mitbringen. Aufschlussreich ist in diesem Zusammenhang, dass der Anteil derer, die berufliche Vorerfahrungen in der Kinder- und Jugendarbeit haben, höher liegt als der Anteil der Fachkräfte. Insofern scheint nicht nur das Vorliegen einer einschlägigen formalen Qualifikation Auskunft darüber zu geben, ob fachspezifische Vorkenntnisse vorhanden sind.

Bezogen auf die Daten zur Maßnahme Schulbegleitung finden sich in Stadt und Landkreis Göttingen vergleichbare Ergebnisse hinsichtlich der meist auf mehrere Monate bis ein Jahr beschränkten Vertragslaufzeit bzw. des Bewilligungszeitraums, dem variierenden Umfang der Maßnahme und der überwiegenden Durchführung durch freie Träger (Deger et al., 2015; Dworschak, 2012b; Henn et al., 2014; Lindmeier et al., 2014; Wohlgemuth, 2009). Auch die Verteilung der Schulbegleitungen über die Schulformen (der hohe Anteil an Grundschulen, der geringe Anteil am Gymnasium) entspricht bisherigen Befunden (Deger et al., 2015; Henn et al., 2014; Lindmeier et al., 2014). Wie vorherige Untersuchungen zeigt auch die vorliegende, dass Schulbegleiter/innen häufig unterrichtsbezogene Tätigkeiten übernehmen, die in das Aufgabenfeld von Schule gehören und im Rahmen der Maßnahme Schulbegleitung gesetzlich nicht vorgesehen sind (Beck, 2010; Dworschak 2012b, 2012c; Wohlgemuth, 2009), und dass insbesondere an Förderschulen Unterstützung bei lebenspraktischen Anforderungen geleistet wird (Dworschak, 2012a, 2012b, 2012c). Vergleichbar mit bisherigen quantitativen Befunden ist darüber hinaus die hohe Gesamtzufriedenheit der Schulbegleiter/innen mit der schulischen Einbindung und Zusammenarbeit (Wohlgemuth, 2009; Zauner \& Zwosta, 2014).

Anknüpfend an quantitative und qualitative Ergebnisse zu Schulbegleitung (Deger et al., 2015; Dworschak, 2012b; Lindmeier et al., 2014) zeigen die Ergebnisse der vorliegenden Untersuchung, dass Schulbegleiter/innen im Rahmen ihrer Tätigkeit mit verschiedenen Personengruppen, einschließlich der Eltern des Kindes, in Kontakt stehen. Vor allem aber liefert die Studie differenzierte Erkenntnisse zur Kooperationspraxis von Schulbegleiter/innen und Klassenleitungen, die den Ergebnissen nach als wichtigste Kooperationspartner/innen angesehen werden können, und zum Zusammenhang mit verschiedenen arbeitsbezogenen Merkmalen von Schulbegleiter/innen. Wurden kooperationsspezifische Variablen bislang eher in Form von Abfragen zur globalen Zufriedenheit mit der Zusammenarbeit erhoben (z. B. Zauner \& Zwosta, 2014), zeigen die vorliegenden Ergebnisse, dass die Ausprägung der Kooperation mit der Klassenleitung in Zusammenhang damit steht, inwieweit sich Schulbegleiter/innen emotional entlastet fühlen und in welchem Maße sie wahrnehmen, dass die Klassenleitung ihrer Arbeit Wertschätzung entgegenbringt. Ebenso hängt die Kooperationspraxis mit der Klassenleitung auch damit zusammen, wie sicher Schulbegleiter/innen sich in ihrer Rolle fühlen und wie zufrieden sie mit ihrer Beschäftigung sind. Die vorliegende 
Studie erweitert bisherige Befunde außerdem um die Erkenntnis, dass einfache Kooperation wie Absprachen und Austausch stärker ausgeprägt ist als Kooperation, die durch gemeinsame Reflektion und die Verständigung über arbeitsbezogene Einschätzungen gekennzeichnet ist, und dass vor allem diese Form von Kooperation mit der Wahrnehmung von Wertschätzung zusammenhängt. Eine kausale Wirkung der Kooperation auf die emotionale Entlastung, die wahrgenommene Wertschätzung, die Rollensicherheit und die Arbeitszufriedenheit kann zwar nicht empirisch nachgewiesen werden, da Korrelationen keine Aussage über die Richtung eines Zusammenhangs zulassen. Aufgrund theoretischer Annahmen und bisheriger Forschungsergebnisse aus der Lehrerkooperationsforschung (siehe Kapitel 3) können diese Merkmale aber als Kooperationswirkungen angenommen werden.

Die Ergebnisse der vorliegenden Untersuchung spiegeln Befunde aus der bisherigen Kooperationsforschung insofern wieder, als dass sie Zusammenhänge von Kooperation mit emotionaler Entlastung und Arbeitszufriedenheit zeigen und eine höhere Ausprägung einfacher Kooperationstätigkeiten gegenüber anspruchsvolleren Kooperationstätigkeiten (z. B. Dizinger, 2015; Drossel \& Willems, 2014; Fussangel, 2008; Richter \& Pant, 2016). Der Erkenntnisgewinn der Untersuchung für die Kooperationsforschung an Schulen liegt vor allem darin, dass sie den Forschungsstand um Ergebnisse zur Zusammenarbeit mit einer Akteursgruppe, die bislang nicht in den Fokus der Kooperationsforschung gerückt ist, erweitert. Die bisherige Forschung zeigt, dass insbesondere multiprofessionelle Kooperation an Schulen mit vielfältigen Herausforderungen verbunden ist (Brähler, 1991; Breuer, 2015; Gebhard et al., 2014; Lehmann-Wermser et al., 2010; Olk et al., 2011). Zuständigkeitsbereiche sind oft nicht klar festgelegt, sondern müssen von den Beteiligten selbst ausgehandelt werden (z. B. Breuer, 2015). Unterschiedliche Verständnisse der eigenen Professionsrolle im Kontext von Bildung und Erziehung sowie die häufige Statusungleichheit der Kooperationspartner/innen können die Zusammenarbeit zusätzlich erschweren, sodass die Zusammenarbeit an Schulen oft auf ein wenig verzahntes Nebeneinander hinausläuft (Böttcher et al., 2011; Olk et al., 2011). Insbesondere komplexere Formen von Kooperation sind an Schulen nicht in ausgeprägtem Maße vorhanden (z. B. Fussangel, 2008) und insgesamt findet wenig unterrichtsbezogene Kooperation zwischen Lehrkräften sowie zwischen Lehrkräften und weiterem pädagogisch tätigen Personal statt (Böttcher et al., 2011; Fussangel, 2008; Steiner \& Tillmann, 2011; Steinert et al., 2006). Vor diesem Hintergrund stellt die vorliegende Untersuchung eine wichtige Ergänzung der bisherigen Kooperationsforschung dar. Schulbegleiter/innen sind durchgängig im Unterricht anwesend, sodass unterrichtsbezogene Kooperation unumgänglich ist. Die mangelnde Konzeption der Maßnahme Schulbegleitung sowie die Schwierigkeit der Trennung der Aufgaben, die Schulbegleitung leisten soll, von den Aufgaben, die Schule leisten soll, stellen an die Kooperation zwischen Lehrkräften und Schulbegleiter/innen besondere Anforderungen. Während es bei der Zusammenarbeit mit dem weiteren pädagogisch tätigen Personal möglich ist, Zuständigkeitsbereiche festzulegen und somit die eigene Autonomie bei der Berufsausübung in hohem Maße zu wahren, ist dies bei der Zusammenarbeit mit Schulbegleiter/innen weniger leicht möglich. In diesem Zusammenhang kommt dem Befund der vorliegenden Untersuchung, dass die Ausprägung der Kooperation mit der Ausprägung von Rollensicherheit zusammenhängt, eine hohe Bedeutung zu. Vor dem Hintergrund, dass im Kontext multiprofessioneller Kooperation häufig die fehlende gegenseitige Anerkennung als belastendes Moment thematisiert wird (z. B. Brähler, 1991), ist auch das Ergebnis, dass die Ausprägung von Kooperation mit der Wahrnehmung von Wertschätzung und mit emotionaler Entlastung zusammenhängt, von großer Wichtigkeit. Aufschlussreich ist in diesem Zusammenhang, dass vor allem anspruchsvolle Kooperation im Sinne gemeinsamer Reflektion stark mit der Wahrnehmung von Wertschätzung zusammenhängt.

\subsection{Implikationen für weitere Forschung und für die schulische Praxis}

Aus den Ergebnissen der vorliegenden Untersuchung ergeben sich verschiedene Implikationen für die weitere Forschung zu Schulbegleitung und zu Kooperation an Schulen sowie für die Weiterentwicklung der Maßnahme Schulbegleitung und die schulische Praxis.

Die Forschungslücke, die zu Schulbegleitung besteht, konnte durch die Untersuchung nur ein kleines Stück weit geschlossen werden, sodass hier weiterhin großer Forschungsbedarf besteht. Die Ergebnisse der Studie zeigen, dass der Kooperation mit der Klassenleitung eine hohe Bedeutung zukommt, lassen aber, auch angesichts der geringen Stichprobengröße, viele Fragen zur Einbindung von Schulbegleiter/innen in das Gesamtsystem Schule offen. Insbesondere multiperspektivisch angelegte Untersuchungen könnten nä- 
heren Aufschluss darüber geben, wie sich die Kooperation mit Schulbegleiter/innen aus Sicht der verschiedenen Akteur/innen gestaltet. Eine offene Frage ist auch, welche Wirkung Schulbegleitung auf die begleiteten Schüler/innen hat und welche Rolle die Kooperation von Schulbegleiter/innen und Lehrkräften dabei spielt. Zur Beantwortung dieser Frage sind insbesondere Längsschnittstudien wie die umfassende Evaluationsstudie von Blatchford et al., (2009b) geeignet. Darüber hinaus ist auch anzuregen, dass qualitative Forschung sich dem Thema Schulbegleitung stärker annimmt, um differenzierte Erkenntnisse über die Ausgestaltung der Kooperation von Lehrkräften und Schulbegleiter/innen zu gewinnen.

Für die schulbezogene Kooperationsforschung ergeben sich aus der vorliegenden Untersuchung verschiedene Anknüpfungspunkte insbesondere zur Untersuchung multiprofessioneller Kooperation. Die gefundenen Zusammenhänge der Kooperationsausprägung mit der Ausprägung von emotionaler Entlastung, Wertschätzung, Rollensicherheit und Arbeitszufriedenheit weisen auf die Bedeutung der Ausgestaltung der Kooperation auf Personenebene hin und verdienen weitere Untersuchung. Da sich die Zusammenhänge nicht kausal interpretieren lassen, sind insbesondere Interventionsstudien anzuregen um Aussagen darüber treffen zu können, ob z. B. Kooperation tatsächlich die Rollensicherheit erhöht oder ob die Rollensicherheit das Eingehen von Kooperation beeinflusst. Aufschlüsse über die kausalen Zusammenhänge würde die Entwicklung gezielter Maßnahmen ermöglichen, um die schulische Kooperationspraxis zu verbessern und einen Arbeitskontext zu schaffen, in dem die Beteiligten sich ihrer Rolle sicher fühlen, Wertschätzung erfahren und emotionale Entlastung und Zufriedenheit erleben. Insbesondere ist anzuregen, dass zwischen den verschiedenen Forschungssträngen zur schulischen Praxis stärkere Verknüpfungen vorgenommen werden. Die Fachdiskurse der allgemeinen Erziehungswissenschaft, die sich hinsichtlich schulischer Kooperation vor allem auf die Erforschung von Ganztagsschulen konzentriert, und der Sonderpädagogik, die sich auf die Zusammenarbeit im inklusiven Unterricht konzentriert und innerhalb derer auch Untersuchungen zu Schulbegleitung angesiedelt sind, scheinen teilweise noch relativ unverbunden. Eine stärkere Bezugnahme aufeinander ist insbesondere im Hinblick auf die Umsetzung von Inklusion im Bildungssystem dringend geraten.

Für die schulische Praxis lässt sich aus den Ergebnissen der Studie zum einen ableiten, dass die Kooperation mit Schulbegleiter/innen inzwischen für viele Lehrkräfte zum Unterrichtsalltag gehört, und zum anderen, dass der spezifischen Ausgestaltung dieser Kooperation hohe Bedeutung zukommt. Vor diesem Hintergrund stellt sich die Frage, welche Kompetenzen Lehrkräfte benötigen, um mit anderen Berufsgruppen professionell zu kooperieren. Eine Herausforderung bildet dabei insbesondere die häufige Zuständigkeit für die Anleitung und Supervision der Schulbegleiter/innen. Da Kooperation lange kein selbstverständlicher Teil des Lehrerberufs war, ist davon auszugehen, dass der überwiegende Teil der bereits berufstätigen Lehrkräfte weder in der ersten noch in der zweiten Phase ihrer Lehramtsausbildung hinreichend auf (multiprofessionelle) Kooperation vorbereitet wurde. Auch wenn die aktuellen Empfehlungen der Kultusministerkonferenz (2014) zur Lehrerbildung Kooperation thematisieren, kann nicht von einer systematischen Ausbildung zur Befähigung professioneller Kooperation die Rede sein (vgl. Steiner \& Tillmann, 2011). Aus diesem Grund scheinen Maßnahmen zur Entwicklung professioneller Kooperationskompetenz auf drei Ebenen ratsam. Kooperation als schulisches Handlungsfeld muss stärker in den Inhalten der universitären Lehramtsausbildung und in der praktischen Ausbildung in der zweiten Phase der Lehramtsausbildung verankert werden. Zudem sollte den bereits berufstätigen Lehrkräften die Möglichkeit gegeben werden, in Fortbildungen die entsprechenden Kompetenzen zu erwerben. Insbesondere von der Durchführung gemeinsamer Fortbildungen für Lehrkräfte und Vertreter/innen anderer an der Schule tätiger Berufsgruppen können dabei wichtige Impulse für die Gestaltung der schulischen Kooperationskultur ausgehen. Auch wenn die bisherige Forschung die Bedeutung von Personenmerkmalen für die Ausgestaltung von Kooperation betont und die Grenzen zwischen den pädagogisch arbeiteten Professionen weniger strikt gezogen sind als noch vor zwei Jahrzehnten (vgl. Rahm et al., 2015), ist die Implementierung der Ausbildung von Kooperationskompetenz dringend geraten, um professionelle Standards in der Kooperation zu gewährleisten (vgl. Putnam \& Borko, 2000). Auf die Frage danach, welche Art von Kompetenzen nötig ist, um professionell zu kooperieren, bieten die theoretischen Analysen zum Zusammenhang von Kooperation und Professionalisierung wichtige Ansatzpunkte und auch die Ergebnisse der vorliegenden Studie liefern hierfür Hinweise. Auf dieser Basis lässt sich die Frage dahingehend beantworten, dass insbesondere die Fähigkeit zur Reflektion der eigenen Berufsrolle und die Bereitschaft, in einen fachlichen Dialog mit anderen Professionen zu treten, der von gegenseitiger Anerkennung geprägt ist, von großer Bedeutung für gelingende (multiprofessionelle) Kooperation sind. Im Fall der Zusammenarbeit mit Schulbegleiter/innen kann das heißen, sich auf die schülerbezogene Perspektive der Schulbegleitung einzulassen, gemeinsam zu überlegen, wie die soziale 
Eingliederung einzelner Schüler/innen gelingen kann und dabei auch zuzulassen, dass Veränderungen der eigenen Berufspraxis notwendig werden.

Obwohl die große Mehrheit der befragten Schulbegleiter/innen sich zufrieden mit der Zusammenarbeit in ihrer Schule zeigt, zeigen die Ergebnisse auch, dass Schulbegleiter/innen in vielen Fällen nicht systematisch eingebunden sind und z. B. nicht an Konferenzen teilnehmen. Um die Kooperation mit Schulbegleiter/innen institutionell zu gewährleisten, bräuchte es sowohl ausgearbeitete Standards für die Einbindung von Schulbegleiter/innen wie auch funktionierende schulinterne Kooperationsstrukturen, die die Einhaltung der Standards ermöglichen und absichern. Bereits bestehende Kooperationsstrukturen zwischen Lehrkräften oder mit weiteren Professionen könnten für die Zusammenarbeit mit Schulbegleiter/innen genutzt werden, ebenso könnte die Implementierung von Kooperationsstrukturen mit Schulbegleiter/innen für weitere kooperative Schulentwicklungsmaßnahmen fruchtbar gemacht werden.

\subsection{Implikationen für die Maßnahme Schulbegleitung}

Wie zuvor bereits ausgeführt, wird die Maßnahme Schulbegleitung in ihrer jetzigen Form kontrovers diskutiert. Die häufig vorgebrachten Kritikpunkte spiegeln sich auch in den Ergebnissen der Studie wider, so z. B. die ungünstigen Arbeitsbedingungen: Zwar zeigen die Schulbegleiter/innen sich insgesamt zufrieden mit ihrer Arbeit, nicht aber mit ihrem vertraglichen Beschäftigungsverhältnis. Auch die Schwierigkeit der Abgrenzung von schulischen Aufgaben und Aufgaben der Eingliederungshilfe findet sich in den Ergebnissen, ebenso wie die fehlende strukturelle Einbindung von Schulbegleiter/innen. Die Ergebnisse legen nahe, dass auch hierzulande ein Teil der unterrichtlichen Instruktion von nicht ausgebildeten Assistenzkräften geleistet wird. Wie die Erfahrungen anderer Länder zeigen (z. B. Blatchford et al., 2009b; Giangreco, 2013), ist ein solcher Assistenzseinsatz insbesondere für den Lernfortschritt der begleiteten Schüler/innen nicht unproblematisch. Zu Recht wird deshalb eine „schleichende Deprofessionalisierung der pädagogischen Fachkräfte im Kontext inklusiver Beschulung“ (Heinrich \& Lübeck, 2013, S. 94) befürchtet, die einem „double standard“" (Giangreco, 2003, S. 53) entspräche: Schüler/innen mit Behinderung würden entsprechend eine weniger professionelle Instruktion erhalten als Schüler/innen ohne Behinderung oder in anderen Worten: „[...] we are assigning the least qualified personnel to students who present the most complex learning challenges" (Giangreco, 2013, S. 5).

Dabei muss für den hiesigen Kontext beachtet werden, dass der Assistenzeinsatz durch Schulbegleitung außerhalb des Kultusbereichs geregelt ist. Schulbegleitung ist keine Maßnahme, die für die flächendeckende Umsetzung von inklusiver Beschulung geplant und konzeptionell ausgearbeitet ist, sondern ist aus dem individuellen Hilferecht der Sozialgesetzgebung entstanden. Insofern ist es wenig verwunderlich, dass die Maßnahme keine passgenaue Lösung für die Herausforderungen inklusiver Beschulung bietet. Dass sie dennoch in so hohem Ausmaße in Anspruch genommen wird, verweist auf den Bedarf an personeller Unterstützung, den einige Schüler/innen benötigen und der offenbar nicht in ausreichendem Maße durch schulisches Personal geleistet werden kann. Bei aller berechtigten Kritik an der Maßnahme Schulbegleitung muss auch gesehen werden, dass sie Inklusion zurzeit oft erst ermöglicht. Eine kurzfristige Ablösung dieser Praxis ist deshalb nicht wünschenswert. Zwar scheinen aufgrund des aktuellen Forschungsstandes Konzepte vorteilhafter, die ohne einzelfallbezogene Hilfen auskommen. Bis zu ihrer Erprobung und flächendeckenden Umsetzung ist es aber keine Option, Schulbegleitung als suboptimales Übergangsphänomen zu betrachten, das die Mühen einer Verbesserung nicht wert ist. Damit wäre den derzeit begleiteten Schüler/innen nicht geholfen. Es scheint deshalb angebracht, zwei parallele Strategien zu verfolgen.

Zum einen müssen kurzfristig Lösungen gefunden werden, Schulbegleitung so zu gestalten, dass sie innerhalb ihrer jetzigen Rahmenbedingungen so gut wie möglich ist. Hierzu braucht es verbindliche Konzepte, damit das Gelingen von Inklusion im Einzelfall nicht vom Engagement individueller Helfer/innen abhängt, die unter ungünstigen Bedingungen arbeiten und möglicherweise wenig fachliche Vorbereitung und Begleitung erfahren. Bei der Entwicklung von Konzepten und Standards helfen die empirisch fundierten Leitlinien zum Einsatz von Assistenzkräften, die von Webster et al. (2013b) entwickelt wurden. Wichtige Eckpunkte sind die Konzeptualisierung der Rolle von Assistenzkräften, fachliche Qualifizierung und Fortbildung sowie regelmäßige Kooperationstreffen und Supervision durch Lehrkräfte. Aus der Praxis heraus gibt es bereits eine Vielzahl von Versuchen, die Qualität von Schulbegleitung zu verbessern. Regional werden in Zusammenarbeit von Schulen, Ämtern und Trägern der Jugend-/Sozialhilfe bereits ausgearbeitete Modelle erprobt und umgesetzt. In Göttingen erprobt das Jugendamt Landkreis in Kooperation mit der 
Evangelischen Jugendhilfe Obernjesa e.V. seit dem Schuljahr 2015/16 eine andere Möglichkeit der Begleitung unterstützungsbedürftiger Schüler/innen, die inzwischen auch vom Jugendamt Stadt mitgetragen wird. Im Pilotprojekt Familienklasse verbringen 5 bzw. 6 Kinder mit emotionalen oder sozialen Verhaltensauffälligkeiten alle zwei Wochen den Vormittag nicht im Klassenverband, sondern zusammen mit einer Lehrkraft und jeweils einem ihrer Erziehungsberechtigen in einer Familienklasse. Dort arbeitet ein in der MultiFamilien Arbeit nach Eia Asen ausgebildeter Pädagoge mit den Familien. Ziel ist die Stärkung der elterlichen Erziehungskompetenz durch gegenseitige Unterstützung und die Einbeziehung der Kinder in den Problemlöseprozess. Konkret geschieht dies, indem zu jedem Termin Verhaltens- und Leistungsziele des Kindes festgelegt werden, deren Erfüllung durch die Lehrkräfte überprüft wird und beim nächsten Termin von allen Teilnehmenden der Familienklasse gewürdigt wird. Zudem werden in zwei Arbeitsphasen zum einen schulische Aufgaben bearbeitet, die die jeweiligen Lehrkräfte zuvor bereitgestellt haben, und zum anderen Gruppenübungen aus der MultiFamilienArbeit durchgeführt und ausgewertet. Bislang ist das Projekt auf die Jahrgangsstufen 5 bis 7 der IGS Bovenden beschränkt; aufgrund der positiven Resonanz ist eine Ausweitung insbesondere auf Grundschulen geplant. Die Jugendämter verstehen Familienklassen als Alternative zu Schulbegleitung, die durch die Zusammenarbeit mit den Eltern nachhaltigere Veränderungen bewirken soll.

Projekte wie diese bieten Ansatzpunkte, um - zum anderen - langfristig umzusetzende, umfassende Konzepte inklusiver Beschulung zu entwickeln, um allen Schüler/innen unabhängig von extern durch die Erziehungsberechtigten zu beantragenden individuellen Hilfemaßnahmen (siehe hierzu Dworschak, 2015) gerecht zu werden. Zur Entwicklung inklusiver Konzepte bietet sich eine Zusammenarbeit mit den Ämtern und Trägern der Jugend-/Sozialhilfe sowie engagierten Schulbegleiter/innen an um die Erfahrungswerte, die mit Schulbegleitung und in entsprechenden Modellversuchen gesammelt wurden, zu nutzen. Ob Schulbegleitung in einer veränderten Form Teil einer langfristigen Konzeptualisierung ist oder ob sich gänzlich anders gestaltete Instrumente besser eignen, kann an dieser Stelle nicht entschieden werden. Hierzu bedarf es weiterer Forschung etwa zur Wirkung verschiedener Unterstützungssysteme auf die Schüler/innen. Eine offene Frage bleibt auch, welche Rolle die Jugend- bzw. Sozialhilfe im schulischen Inklusionsprozess langfristig spielen soll und wird. Der Integrationsanspruch, der an Schule heute gestellt wird, beschränkt sich nicht auf die Teilhabe an formaler Bildung. Unter anderem durch die UN-Behindertenrechtskonvention ist auch die soziale Teilhabe in den Fokus schulischer Reformprozesse gerückt. Ihre Gewährleistung erfolgt zurzeit durch Schulbegleitung; wenn Inklusion ernstgemeint ist, muss soziale Teilhabe als schulische Kernaufgabe definiert und Schulen dazu befähigt werden, dieser Aufgabe gerecht zu werden. Dazu braucht es die Bereitstellung entsprechender personeller und finanzieller Ressourcen sowie die Verankerung entsprechender Ausbildungs- und Weiterbildungsinhalte.

Abschließend lässt sich festhalten, dass die zentrale Frage nach der Funktion, die Eingliederungshilfe im schulischen Kontext erfüllen kann oder soll, weiterhin bestehen bleibt und im Zuge der Ausweitung inklusiver Beschulung an Dringlichkeit gewinnen wird. Zur Beantwortung damit eingehender Fragen wie der Qualifizierung von Schulbegleiter/innen, der Aufgaben- und Rollendefinition, der Kooperation mit den Lehrkräften und nicht zuletzt der Frage nach den Wirkungen von Schulbegleitungen auf Schülervariablen bedarf es dringend weiterer Forschung. 


\section{Literaturverzeichnis}

AGJ (2014). Schulbegleitung allein kann kein inklusives Schulsystem gewährleisten. Diskussionspapier der Arbeitsgemeinschaft für Kinder- und Jugendhilfe. Gemeinsam leben, 22 (4), 230-232.

Ahlgrimm, F. (2012). Wirkungen von Zusammenarbeit auf das Selbstbild und die professionelle Entwicklung von Lehrkräften. In S. G. Huber \& F. Ahlgrimm (Hrsg.), Kooperation. Aktuelle Forschung zur Kooperation in und zwischen Schulen sowie mit anderen Partnern (S. 159-183). Münster: Waxmann.

Ahlgrimm, F., Krey, J. \& Huber, S. G. (2012). Kooperation - was ist das? Implikationen unterschiedlicher Begriffsverständnisse. In S. G. Huber \& F. Ahlgrimm (Hrsg.), Kooperation. Aktuelle Forschung zur Kooperation in und zwischen Schulen sowie mit anderen Partnern (S. 17-29). Münster: Waxmann.

Altrichter, H. (2000). Konfliktzonen beim Aufbau schulischer Qualitätssicherung und Qualitätsentwicklung. In A. Helmke, W. Hornstein \& E. Terhart (Hrsg.), Qualität und Qualitätssicherung im Bildungsbereich: Schule, Sozialpädagogik, Hochschule (Zeitschrift für Pädagogik, 41, Beiheft, S. 93-110). Weinheim: Beltz.

Appel, S., Ludwig, H., Rother, U. \& Rutz, G. (Hrsg.) (2003). Jahrbuch Ganztagsscbule 2004: Neue Chancen für die Bildung. Schwalbach: Wochenschau Verlag.

Argyle, M. (1991). Cooperation. The basis of sociability. London: Routledge.

Arndt, A.-K., Harting, A., Katzer, P., Laubner, M., Stenger, S. \& Werning, R. (2014). Inklusiver Unterricht. Leitideen zur Organisation und Kooperation (Schulmanagement-Handbuch, Bd.152). München: Oldenbourg.

Arnoldt, B. (2008a). Kooperationsformen - Bedingungen für gelingende Zusammenarbeit? In H. G. Holtappels, E. Klieme, T. Rauschenbach \& L. Stecher (Hrsg.), Ganstagsschule in Deutschland. Ergebnisse der Ausgangserbebung der "Studie zur Entwicklung von Ganætagsschulen“ (StEG) (Studien zur ganztägigen Bildung, 2., korr. Aufl., S. 123-136). Weinheim: Juventa.

Arnoldt, B. (2008b). Öffnung von Ganztagsschule. In H. G. Holtappels, E. Klieme, T. Rauschenbach \& L. Stecher (Hrsg.), Ganztagsschule in Deutschland. Ergebnisse der Ausgangserbebung der „Studie zur Entwicklung von Ganztagsschulen" (StEG) (Studien zur ganztägigen Bildung, 2., korr. Aufl., S. 86-105). Weinheim: Juventa.

Autorengruppe Bildungsberichterstattung (Hrsg.) (2014). Bildung in Deutscbland 2014. Ein indikatorengestützter Bericht mit einer Analyse zur Bildung von Menschen mit Bebinderungen. Bertelsmann: Bielefeld. Verfügbar unter http:/ /www.bildungsbericht.de/de/bildungsberichte-seit-2006/bildungsbericht2014/pdf-bildungsbericht-2014/bb-2014.pdf [Abgerufen am 30.05.2016].

Azad, G. F., Locke, J., Downey, M. M., Xie, M. \& Mandell, D. S. (2015). One-to-One Assistant Engagement in Autism Support Classrooms. Teacher education and special education: The journal of the teacher education division of the council for exceptional children, 38 (4), 337-346.

Bacher, J., Pfaffenberger, M. \& Pöschko, H. (2007). Arbeitssituation und Weiterbildungsbedarf von Schulassistent/innen. Endbericht (korr. Fassung). Verfügbar unter http://www.forschungsnetzwerk.at/downloadpub/schulassistenz_jugendliche_mit_foerderbedarf_ooe_2007.pdf [Abgerufen am 30.05.2016].

Banafsche, M. (2013). Kinder und Jugendliche mit Behinderung zwischen SGB VIII und SGB XII - im Lichte der UN-Behindertenrechtskonvention. In C. Dorrance \& C. Dannenbeck (Hrsg.), Doing inclusion. Inklusion in einer nicht inklusiven Gesellschaft (S. 42-57). Bad Heilbrunn: Klinkhardt.

Barth, A.-R. (1997). Burnout bei Lehrern. Theoretische Aspekte und Ergebnisse einer Untersucbung (2., unveränd. Aufl.). Göttingen: Hogrefe. 
Bauer, K.-O. (2008). Lehrerinteraktion und -kooperation. In W. Helsper \& J. Böhme (Hrsg.), Handbuch der Schulforschung (2., durchges. u. erw. Aufl., S. 839-856). Wiesbaden: VS Verlag für Sozialwissenschaften.

Baumert, J., Masuhr, V., Möller, J., Riecke-Baulecke, T., Tenorth, H.-E. \& Werning, R. (2013). Inklusion. Forschungsergebnisse und Perspektiven (Schulmanagement-Handbuch, Bd. 146). München: Oldenbourg.

Beck, C., Dworschak, W. \& Eibner, S. (2010). Schulbegleitung am Förderzentrum mit dem Förderschwerpunkt geistige Entwicklung. Zeitschrift für Heilpädagogik, 61 (7), 244-254.

Blatchford, P., Bassett, P., Brown, P., Koutsoubou, M., Martin, C., Russell, A. et al. (2009a). Deployment and impact of support staff in schools. The impact of support staff in schools (Results from strand 2, wave 2). Institute of Education, University of London. Verfügbar unter http://dera.ioe.ac.uk/10818/1/DCSFRR148.pdf [Abgerufen am 30.05.2016].

Blatchford, P., Bassett, P., Brown, P., Martin, C., Russell, A. \& Webster, R. (2009b). Deployment and impact of support staff in schools. Characteristics, working conditions and job satisfaction of support staff in schools (Strand 1, waves 1-3 in 2004, 2006, 2008). Institute of Education, University of London. Verfügbar unter http://maximisingtas.co.uk/assets/content/disss1w123r.pdf [Abgerufen am 30.05.2016].

Blatchford, P., Bassett, P., Brown, P., Martin, C., Russell, A. \& Webster, R. (2009c). Deployment and Impact of Support Staff Project (Research Brief). Institute of Education, University of London. Verfügbar unter http://maximisingtas.co.uk/assets/content/dissressum.pdf [Abgerufen am 30.05.2016].

Blatchford, P., Webster, R. \& Russell, A. (2012). Challenging the role and deployment of teaching assistants in mainstream schools: The impact on schools. Final report on the Effective Employment of Teaching Assistants (EDTA) project. Verfügbar unter http://www.zenamartin.co.uk/upload/edtareport-2.pdf [Abgerufen am 30.05.2016].

Bloemer-Hausmanns, S. (2014). Integrationshilfe - eine Baustellenbesichtigung. Gemeinsam leben, 22 (4), 226-229.

Blömeke, S., Reinhold, P., Tulodziecki, G. \& Wildt, J. (Hrsg.) (2004). Handbuch Lehrerbildung. Bad Heilbrunn/Obb.: Klinkhardt.

Boban, I. \& Hinz, A. (2003). Index für Inklusion, Martin-Luther-Universität Halle-Wittenberg. Halle-Wittenberg.

Boban, I. \& Hinz, A. (2008). Sonderpädagogische Förderung in der Allgemeinen Schule (Integration) und in Sonderschulen. In K.-H. Arnold, O. Graumann \& A. Rakhkochkine (Hrsg.), Handbuch Förderung. Grundlagen, Bereiche und Methoden der individuellen Förderung von Schülern (S. 410-419). Weinheim: Beltz.

Böhm-Kasper, O. (2004). Schulische Belastung und Beanspruchung. Eine Untersuchung von Schülern und Lehrern am Gymnasium (Pädagogische Psychologie und Entwicklungspsychologie, Bd. 43). Münster: Waxmann.

Böhm-Kasper, O., Gräsel, C. \& Weishaupt, H. (2009). Schulische Beanspruchung und Kooperation - Ein Projekt zum Beanspruchungserleben und Formen der Lehrerkooperation an Halb-und Ganztagsschulen in Nordrhein-Westfalen - Ergebnisse der ersten schriftlichen Befragung, Wuppertal.

Böing, U. (2013). Schritte inklusiver Schulentwicklung. Erkenntnisse für die barrierefreie Teilhabe hochgradig sebbebinderter und blinder Kinder und Jugendlicher an inklusiven Bildungsprozessen. Würzburg: Bentheim.

Boller, S. (2012). Multiprofessionalität als Weg der Schulentwicklung? Möglichkeiten und Grenzen berufsübergreifender Zusammenarbeit in der Schule. In S. G. Huber \& F. Ahlgrimm (Hrsg.), Kooperation. Aktuelle Forschung zur Kooperation in und zwischen Schulen sowie mit anderen Partnern (S. 203-221). Münster: Waxmann.

Bondorf, N. (2013). Profession und Kooperation. Eine Verhältnisbestimmung am Beispiel der Lehrerkooperation. Wiesbaden: Springer Fachmedien.

Bonsen, M. \& Rolff, H.-G. (2006). Professionelle Lerngemeinschaften von Lehrerinnen und Lehrern. Zeitschrift für Pädagogik, 52 (2), 167-184. 
Booth, T. \& Ainscow, M. (2002). Index for inclusion. Developing learning and participation in schools. Centre for Studies on Inclusive Education. Verfügbar unter http://csie.org.uk/resources/translations/IndexEnglish.pdf [Abgerufen am 30.05.2016].

Bortz, J. \& Döring, N. (2006). Forschungsmethoden und Evaluation für Human- und Sozialwissenschaftler (4., überarb. Aufl.). Heidelberg: Springer.

Bortz, J. \& Schuster, C. (2010). Statistik für Human- und Sozialwissenschaftler (Springer-Lehrbuch, 7., vollst. überarb. u. erw. Aufl.). Berlin, Heidelberg: Springer.

Bos, W., Strietholt, R., Goy, M., Stubbe, T. C., Tarelli, I., Hornberg, S. et al. (2010). IGLU 2006. Dokumentation der Erbebungsinstrumente. Münster: Waxmann.

Böttcher, W., Maykus, S., Altermann, A. \& Liesegang, T. (2011). Multiprofessionelle Kooperation an Ganztagsschulen. In K. Speck, T. Olk, O. Böhm-Kasper, H.-J. Stolz \& C. Wiezorek (Hrsg.), Ganztagsschulische Kooperation und Professionsentwicklung. Studien zu multiprofessionellen Teams und sozialräumlicher Vernetzung (Studien zur ganztägigen Bildung, S. 102-113). Weinheim: Juventa.

Brähler, H. (1991). Schüler mit Lernbehinderungen in der Allgemeinen Schule. Kooperationsmodell Baden-Württemberg (Europäische Hochschulschriften, Reihe XI Pädagogik, Bd. 470). Frankfurt a. M.: Peter Lang.

Breuer, A. (2011). Lehrer-Erzieher-Teams - Kooperation als Differenzierung von Zuständigkeit. In K. Speck, T. Olk, O. Böhm-Kasper, H.-J. Stolz \& C. Wiezorek (Hrsg.), Ganztagsschulische Kooperation und Professionsentwicklung. Studien zu multiprofessionellen Teams und sozialräumlicher Vernetzung (Studien zur ganztägigen Bildung, S. 85-101). Weinheim: Juventa.

Breuer, A. (2015). Lehrer-Erzieher-Teams an ganztägigen Grundschulen. Kooperation als Differenzierung von Zuständigkeiten (Schule und Gesellschaft, Bd. 59). Wiesbaden: Springer VS.

Bühner, M. (2011). Einführung in die Test- und Fragebogenkonstruktion (PS Psychologie, 3., akt. u. erw. Aufl.). München: Pearson Studium.

Bundschuh, K. \& Baier, S. (2009). Sonderschulwesen und sonderpädagogische Einrichtungen. In S. Blömeke, T. Bohl, L. Haag, G. Lang-Wojtasik \& W. Sacher (Hrsg.), Handbuch Schule. Theorie - Organisation - Entwicklung (UTB Schulpädagogik, Bd. 8392, S. 288-298). Bad Heilbrunn: Klinkhardt.

Burns, D. \& Darling-Hammond, L. (2014). Teaching around the world: What can TALIS tell us? Stanford: Center for Opportunity Policy in Education. Verfügbar unter https://edpolicy.stanford.edu/sites/default/files/publications/teaching-around-world-what-can-talis-tell-us_3.pdf [Abgerufen am 30.05.2016].

Butt, R. \& Lowe, K. (2012). Teaching assistants and class teachers. Differing perceptions, role confusion and the benefits of skills-based training. International Journal of Inclusive Education, 16 (2), 207-219.

Chambers, D. (2015). The changing nature of the roles of support staff. In D. Chambers (Ed.), Working with teaching assistants and other support staff for inclusive education (International Perspectives on Inclusive Education, vol. 4, pp. 3-25). Bingley (UK): Emerald.

Conée, S. \& Ranscht, D. (2012). Schulbegleiter im Unterricht. In S. Stangier \& E.-M. Thoms (Hrsg.), Eine Schule für alle. Inklusion umsetzen in der Sekundarstufe (S. 50-53). Mülheim an der Ruhr: Verl. an der Ruhr.

Deger, P., Puhr, K. \& Jerg, J. (2015). Inklusion von Kindern und Jugendlichen mit einer Behinderung in allgemeinen Einrichtungen der Kindertagesbetreuung und Schulen. Eine Untersuchung zur Praxis der Gewährung von Leistungen der Eingliederungshilfe in Baden-Württemberg unter Einbeziehung der strukturellen Rabmenbedingungen von Inklusion (Kommunalverband für Jugend und Soziales Baden-Württemberg, Hrsg.). Verfügbar unter http://www.kvjs.de/fileadmin/dateien/kvjs-forschung/Transfer/Abschlussbericht_Inklusion_Kita-Schule.pdf [Abgerufen am 30.05.2016].

Dieckmann, K., Höhmann, K. \& Tillmann, K. (2008). Schulorganisation, Organisationskultur und Schulklima an ganztätigen Schulen. In H. G. Holtappels, E. Klieme, T. Rauschenbach \& L. Stecher (Hrsg.), 
Ganztagsschule in Deutschland. Ergebnisse der Ausgangserbebung der ,Studie zur Entwicklung von Ganztagsschulen" (StEG) (Studien zur ganztägigen Bildung, 2., korr. Aufl., S. 164-185). Weinheim: Juventa.

Ditton, H. (2000). Qualitätskontrolle und Qualitätssicherung in Schule und Unterricht. Ein Überblick zum Stand der empirischen Forschung. In A. Helmke, W. Hornstein \& E. Terhart (Hrsg.), Qualität und Qualitätssicherung im Bildungsbereich: Schule, Sorialpädagogik, Hochschule (S. 73-92). Zeitschrift für Pädagogik, 41. Beiheft. Weinheim: Beltz.

Dizinger, V. (2015). Professionelle und interprofessionelle Kooperation von Lehrerinnen und Lehrern im Kontext schulischer Belastung und Beanspruchung. Dissertation, Universität Bielefeld.

Dizinger, V. \& Böhm-Kasper, O. (2012). Mixed Methods zur Analyse interprofessioneller Kooperation an Ganztagsschulen. In M. Gläser-Zikuda, T. Seidel, C. Rohlfs, A. Gröschner \& S. Ziegelbauer (Hrsg.), Mixed methods in der empirischen Bildungsforschung [74. Tagung der Arbeitsgruppe Empirische Pädagogische Forschung (AEPF) im September 2010 in Jena] (S. 135-149). Münster: Waxmann.

Dizinger, V., Fussangel, K. \& Böhm-Kasper, O. (2011a). Interprofessionelle Kooperation an Ganztagsschulen aus der Perspektive der Lehrkräfte - Wie lässt sie sich erfassen und wie wird sie im schulischen Belastungs- und Beanspruchungs-Geschehen bewertet? In K. Speck, T. Olk, O. Böhm-Kasper, H.-J. Stolz \& C. Wiezorek (Hrsg.), Ganztagsschulische Kooperation und Professionsentwicklung. Studien zu multiprofessionellen Teams und sozialräumlicher Vernetzung (Studien zur ganztägigen Bildung, S. 114-127). Weinheim: Juventa.

Dizinger, V., Fussangel, K. \& Böhm-Kasper, O. (2011b). Lehrer/in sein an der Ganztagsschule. Neue Kooperationsanforderungen - neue Belastungen? Zeitschrift für Eržiehungswissenschaft, 14 (3), $43-61$.

Drossel, K. \& Willems, A. S. (2014). Zum Zusammenhang von Formen der Lehrerkooperation, des Schulleitungshandelns und des Kooperationsklimas an Ganztagsgymnasien. In K. Drossel, R. Strietholt \& W. Bos (Hrsg.), Empirische Bildungsforschung und evidenzbasierte Reformen im Bildungswesen (S. 129-154). Münster: Waxmann.

Dworschak, W. (2010). Schulbegleitung, Integrationshelfer, Schulassistent? Begriffliche Klärung einer Maßnahme zur Integration in die Allgemeine Schule bzw. die Förderschule. Teilhabe, 49 (3), 131-135.

Dworschak, W. (2012a). Schulbegleitung an Förder- und Allgemeinen Schulen. Divergente Charakteristika einer Einzelfallmaßnahme. Zeitschrift für Heilpädagogik, 63 (10), 414-421.

Dworschak, W. (2012b). Schulbegleitung im Förderschwerpunkt geistige Entwicklung an der allgemeinen Schule. Ergebnisse einer bayrischen Studie im Schuljahr 2010/11. Gemeinsam leben (2), 80-94.

Dworschak, W. (2012c). Schulbegleitung/Integrationshilfe: Ergebnisse einer Studie des Lebenshilfe-Landesverbandes Bayern (Lebenshilfe für Menschen mit geistiger Behinderung - Landesverband Bayern e. V., Hrsg.).

Dworschak, W. (2014). Zur Bedeutung individueller Merkmale im Hinblick auf den Erhalt einer Schulbegleitung. Eine empirische Analyse im Förderschwerpunkt geistige Entwicklung an bayerischen Förderschulen. Empirische Sonderpädagogik (2), 150-171.

Dworschak, W. (2015). Zur Bedeutung von Kontextfaktoren im Hinblick auf den Erhalt einer Schulbegleitung. Eine empirische Analyse im Förderschwerpunkt geistige Entwicklung an bayerischen Förderschulen. Empirische Sonderpädagogik (1), 56-72.

Eberwein, H. (1998). Ein Rückblick nach 25 Jahren Integrationsentwicklung. Die Empfehlungen des Deutschen Bildungsrates ,Zur pädagogischen Förderung behinderter und von Behinderung bedrohter Kinder und Jugendlicher" von 1973. Gemeinsam leben - Zeitschrift für integrative Erziehung, 6 (2), 6164.

Edmonson, S. (2006). Role ambiguity. In F. W. English (Ed.), Encyclopedia of educational leadership and administration. Thousand Oaks, Calif: Sage.

Ehlers, H., Jude, N., Klieme, E. \& Helmke, A. (2008). Soziodemografische und fachdidaktischrelevante Merkmale von Deutsch-Lehrpersonen. In E. Klieme, W. Eichler, A. Helmke, R. H. Lehmann, G. Nold, H.-G. Rolff et al. (Hrsg.), Unterricht und Kompetenzerwerb in Deutsch und Englisch. Ergebnisse der DESI-Studie (S. 313-318). Weinheim: Beltz. 
Ernst, K.-F. (2013). Behinderung. Alle Leistungen und Rechte, die Ihnen zustehen (ARD-Ratgeber Recht). Düsseldorf: Verbraucherzentrale NRW.

Esslinger, I. (2002). Berufsverständnis und Schulentwicklung: ein Passungsverhältnis? Eine empirische Untersuchung zu schulentwicklungsrelevanten Berufsauffassungen von Lebrerinnen und Lehrern (Klinkhardt Forschung). Bad Heilbrunn/Obb.: Klinkhardt.

Farrell, P., Alborz, A., Howes, A. \& Pearson, D. (2010). The impact of teaching assistants on improving pupils' academic achievement in mainstream schools. A review of the literature. Educational Review, 62 (4), 435-448.

Fegert, J. M. \& Schepker, R. (2014). Ambivalenzen um die Inklusion. Zeitschrift für Kinder-und Jugendpsychiatrie und Psychotherapie, 42 (5), 297-299.

Fegert, J. M., Ziegenhain, U. \& Besier, T. (2016). Projekt „Schulbegleiter - Entwicklung eines interdisziplinären Curriculums als Beitrag zur Inklusion ". Kinder- und Jugendpsychiatrie/Psychotherapie Universitätsklinikum Ulm. Verfügbar unter http://www.bwstiftung.de/fileadmin/Mediendatenbank_DE/BW_Stiftung/Programme_Dateien/Gesellschaft_u_Kultur/Gesundheit_Inklusion/Schulbegleiter/Zwischenstand_Schulbegleiter.pdf [Abgerufen am 30.05.2016].

Fischer, J. (2011). Integration von Kindern miterhöhtem Förderbedarf in Schule und Jugendhilfe. Ein Ansatz zur Vernetzung von kommunaler Bildungs- und Sozialpolitik. In S. Börner, T. Buchholz \& J. Fischer (Hrsg.), Gemeinsamer Unterricht in Thüringen -Bilanz und Perspektiven. Tagungsband des 5. Landesweiten Integrationstages Thüringen 2010 (S. 70-77).

Fisher, M. \& Pleasants, S. L. (2012). Roles, responsibilities, and concerns of paraeducators. Findings from a statewide survey. Remedial and special education, 33 (5), 287-297.

Frey, A. (2014). Kompetenzmodelle und Standards in der Lehrerbildung und im Lehrerberuf. In E. Terhart, H. Bennewitz \& M. Rothland (Hrsg.), Handbuch der Forschung zum Lehrerberuf (2., überarb. u. erw. Aufl., S. 712-744). Münster: Waxmann.

Furthmüller, P. (2014). Skalenverzeichnis StEG. Skalen und Indizes der Scientific-Use-Files 2005 bis 2009. Verfügbar unter https://www.iqb.hu-berlin.de/fdz/studies/StEG/StEG_SV.pdf [Abgerufen am 30.05.2016].

Fussangel, K. (2008). Subjektive Theorien von Lehrkeäften zur Kooperation. Eine Analyse der Zusammenarbeit von Lehrerinnen und Lehrern in Lerngemeinschaften. Dissertation, Universität Wuppertal.

Fussangel, K. \& Gräsel, C. (2012). Lehrerkooperation aus der Sicht der Bildungsforschung. In E. Baum, T.-S. Idel \& H. Ullrich (Hrsg.), Kollegialität und Kooperation in der Schule. Theoretische Konzepte und empirische Befunde (S. 29-40). Wiesbaden: Springer VS.

Fussangel, K. \& Gräsel, C. (2014). Forschung zur Kooperation im Lehrerberuf. In E. Terhart, H. Bennewitz \& M. Rothland (Hrsg.), Handbuch der Forschung zum Lehrerberuf (2., überarb. u. erw. Aufl., S. 846864). Münster: Waxmann.

Gebhard, S., Happe, C., Paape, M., Riestenpatt, J., Vägler, A., Wollenweber, K. U. et al. (2014). Merkmale und Bewertung der Kooperation von Sonderpädagogen und Regelschullehrkräften in inklusiven Unterrichtssettings. Empirische Sonderpädagogik (1), 17-32.

Giangreco, M. F. (2003). Working with paraprofessionals. Educational Leadership, 61 (2), 50-53.

Giangreco, M. F. (2013). Teacher assistant supports in inclusive schools. Research, practices and alternatives. Australasian journal of special education, 37 (2), 93-106.

Giangreco, M. F. \& Broer, S. M. (2005). Questionable utilization of paraprofessionals ininclusive schools: Are we addressing symptoms or causes? Focus on autism and other developmental disabilities, 20 (1), 10-26.

Giangreco, M. F., Halvorsen, A. T., Doyle, M. B. \& Broer, S. M. (2004). Alternatives to overreliance on paraprofessionals in inclusive schools. Journal of special education leadership, 17 (2), 82-90. 
Giangreco, M. F., Suter, J. C. \& Doyle, M. B. (2010). Paraprofessionals in inclusive schools. A review of recent research. Journal of educational and psychological consultation, 20 (1), 41-57.

Götzinger, R., Richter, G., Scheidle, M., Scheidle, R. \& Wittmann, B. (2012). Wege der integrativen Beschulung eines Jungen mit frühkindlichem Autismus an einem Gymnasium - aus Sicht des Betroffenen, der Mutter, der Schulbegleiterin, des Schulleiters und der Autismusbeauftragten. In H. Sautter, K. Schwarz \& R. Trost (Hrsg.), Kinder und Jugendliche mit Autismus-Spektrum-Störung. Neue Wege durch die Schule (S. 215-222). Stuttgart: Kohlhammer.

Gräsel, C., Fussangel, K. \& Pröbstel, C. (2006). Lehrkräfte zur Kooperation anregen - eine Aufgabe für Sisyphos? Zeitschrift für Pädagogik, 52 (2), 205-219.

Greß, J. (2014). Recht und Förderung für mein behindertes Kind. Elternratgeber für alle Lebensphasen - alles zu Sozialleistungen, Betreuung und Behindertentestament (2. Aufl.). München: C. H. Beck.

Hanover Research (2010). Best practices in utilizing special education assistants. Verfügbar unter https://isminc.com/pdf/research-free/academic-leadership/2717 [Abgerufen am 30.05.2016].

Hedderich, I. \& Hegner, S. (2009). Multiprofessionalität in der Körperbehindertenpädagogik - Zusammenarbeit von Pädagogen und Therapeuten im Auftrag einer ganzheitlichen Förderung. Heilpädagogik online (1), 25-49.

Heinrich, M. \& Lübeck, A. (2013). Hilflos häkelnde Helfer? Zur pädagogischen Rationalität von Integrationshelfer/inne/n im inklusiven Unterricht. Bildungsforschung, 10 (1), 91-110.

Henn, K., Thurn, L., Besier, T., Künster, A. K., Fegert, J. M. \& Ziegenhain, U. (2014). Schulbegleiter als Unterstützung von Inklusion im Schulwesen. Erhebung zur gegenwärtigen Situation von Schulbegleitern in Baden-Württemberg. Zeitschrift für Kinder- und Jugendpsychiatrie und Psychotherapie, 42 (6), 397 403.

Hillenbrand, C. (2012). Kooperation von Jugendhilfe und Schule - Fachwissenschaftliche Aspekte und Brennpunkte. In R. Markowetz \& J. E. Schwab (Hrsg.), Die Zusammenarbeit von Jugendhilfe und Schule. Inklusion und Chancengerechtigkeit zwischen Anspruch und Wirklichkeit (S. 114-126). Bad Heilbrunn: Klinkhardt.

Höhmann, K., Bergmann, K. \& Gebauer, M. (2008). Personal. In H. G. Holtappels, E. Klieme, T. Rauschenbach \& L. Stecher (Hrsg.), Ganztagsschule in Deutschland. Ergebnisse der Ausgangserbebung der ,Studie zur Entwicklung von Ganztagsschulen "(StEG) (Studien zur ganztägigen Bildung, 2., korr. Aufl., S. 77-85). Weinheim: Juventa.

Holtappels, H. G. (2008). Schule und Sozialpädagogik. In W. Helsper \& J. Böhme (Hrsg.), Handbuch der Schulforschung (2., durchges. u. erw. Aufl., S. 495-512). Wiesbaden: VS Verlag für Sozialwissenschaften.

Holtappels, H. G., Lossen, K., Spillebeen, L. \& Tillmann, K. (2011). Schulentwicklung und Lehrerkooperation in Ganztagsschulen. Zeitschrift für Erziehungswissenschaft, 14 (3), 25-42.

Kauper, T., Retelsdorf, J., Bauer, J., Rösler, L., Möller, J., Prenzel, M. et al. (2012). PaLea - Panel zum Lehramtsstudium. Skalendokumentation und Häufigkeitsauszählungen des BMBF-Projektes (1. Welle; Herbst 2009). Kiel: IPN Leipniz-Institut für die Pädagogik der Naturwissenschaften und Mathematik.

Keil, S., Baier, D., Friedemann, A. \& Stange, K.-H. (2010). Modellprojekt zur Qualifizierung von Schulbegleitern und Schaffung von Netzwerken für die gelungene schulische Integration in Thüringen. Erster Zwischenbericht. Berichtzeitraum 01.05.2009-31.12.2009. Erfurt: Institut für Berufsbildung und Sozialmanagement GmbH. Verfügbar unter https://www.fh-erfurt.de/soz/fileadmin/SO/Dokumente/Lehrende/Stange_Karl-Heinz_Prof_Dr/Zwischenbericht_05-2009_12-2009.pdf [Abgerufen am 30.05.2016].

Kißgen, R., Franke, S., Ladinig, B., Mays, D. \& Carlitscheck, J. (2013). Schulbegleitung an Förderschulen in Nordrhein-Westfalen: Ausgangslage, Studienkonzeption und erste Ergebnisse. Empirische Sonderpädagogik (3), 263-276. 
Klemm, K. (2015). Inklusion in Deutschland. Daten und Fakten (Bertelsmann Stiftung, Hrsg.). Verfügbar unter http://www.bertelsmann-stiftung.de/fileadmin/files/BSt/Publikationen/GrauePublikationen/Studie_IB_Klemm-Studie_Inklusion_2015.pdf [Abgerufen am 30.05.2016].

Klieme, E., Rauschenbach, T., Holtappels, H. G. \& Stecher, L. (2015). Ganatagsschule 2014/2015. Deskriptive Befunde einer bundesweiten Befragung. Verfügbar unter http://www.projekt-steg.de/sites/default/files/StEG_Bundesbericht\%202015_final_0.pdf [Abgerufen am 30.05.2016].

KMK (1999). Empfehlungen zum Förderschwerpunkt Unterricht kranker Schülerinnen und Schüler. Verfügbar unter http://www.kmk.org/fileadmin/Dateien/veroeffentlichungen_beschluesse/1998/1998_03_20FS-Kranke-Schueler.pdf [Abgerufen am 30.05.2016].

KMK (2002). PISA 2000 - Zentrale Handlungsfelder. Zusammenfassende Darstellung der laufenden und geplanten Maßnahmen in den Ländern. Verfügbar unter https://www.kmk.org/fileadmin/Dateien/veroeffentlichungen_beschluesse/2002/2002_10_07-Pisa-2000-Zentrale-Handlungsfelder.pdf [Abgerufen am 30.05.2016].

KMK (2004). Standards für die Lebrerbildung: Bildungswissenschaften. Verfügbar unter http://www.kmk.org/fileadmin/Dateien/veroeffentlichungen_beschluesse/2004/2004_12_16Standards-Lehrerbildung.pdf [Abgerufen am 30.05.2016].

KMK (2011). Inklusive Bildung von Kindern und Jugendlichen mit Behinderungen in Schulen. Beschluss der Kultusministerkonferenz vom 20.10.2011. Verfügbar unter http://www.kmk.org/fileadmin/Dateien/veroeffentlichungen_beschluesse/2011/2011_10_20-Inklusive-Bildung.pdf [Abgerufen am 30.05.2016].

KMK (2016). Sonderpädagogische Förderung in Schulen 2005 bis 2014. Statistische Veröffentlichungen der Kultusministerkonferenz, Dokumentation Nr. 210, Februar 2016. Verfügbar unter https://www.kmk.org/filead$\mathrm{min} /$ Dateien/pdf/Statistik/Dokumentationen/Dok_210_SoPae_2014.pdf\#page=6\&zoom=auto,274,247 [Abgerufen am 30.05.2016].

Knaack, I. (2006). Individuelle Förder- und Hilfeplanung als multiprofessionelle Herausforderung aus dem Blickwinkel von Schule. In C. Neuberger (Hrsg.), Dialog der Konzepte: Hilfeplanung nach \36 SGB VIII und in angrenzenden Hilfen. Dokumentation eines ExpertInnengesprächs am DJI vom 17. bis 18. Oktober 2005 (S. 102-115). München: Deutsches Jugendinstitut dji.

Knuf, O. (2013). Von der Schulbegleitung zum Teilhabemanagement. In V. Moser (Hrsg.), Die inklusive Schule. Standards für die Umsetzung (Schulpädagogik, 2. Aufl., S. 93-99). Stuttgart: Kohlhammer.

Köller, O. \& Möller, J. (2006). Selbstwirksamkeit. In D. H. Rost (Hrsg.), Handwörterbuch Pädagogische Psychologie (Schlüsselbegriffe, 3., überarb. und erw. Aufl., S. 693-699). Weinheim: Beltz PVU.

Köpfer, A. (2013a). Inclusion in Canada. Analyse inclusiver Unterrichtsprozesse, Unterstützungsstrukturen und Rollen am Beispiel kanadischer Schulen in den Provinzen New Brunswick, Prince Edward Island und Québec. Bad Heilbrunn: Klinkhardt.

Köpfer, A. (2013b). Inclusive Unterstützungsstrukturen und Rollen am Beispiel kanadischer Schulen in den Provinzen New Brunswick, Prince Edward Island und Québec. Heilpädagogik online (4).

Krämer-Mandeau, W. \& Scharnhorst-Engel, G. (2014). Schulentwicklungsplan Landkereis Göttingen. Verfügbar unter http://www.landkreisgoettingen.de/pics/medien/1_1436860774/SEP_Gutachten_LK_Goettingen_2014_Teil_1.pdf [Abgerufen am 30.05.2016].

Kron, M. (2012). Frühe gemeinsame Bildung und Erziehung von Kindern mit und ohne Behinderung unterschiedliche Systeme und Wege in Europa. In S. Seitz, N.-K. Finnern, N. Korff \& K. Scheidt (Hrsg.), Inklusiv gleich gerecht? Inklusion und Bildungsgerechtigkeit [25. Jahrestagung der Integrations- und Inklusionsforschung in Bremen] (S. 154-159). Bad Heilbrunn: Klinkhardt.

Kuckartz, U.; Rädiker, S., Ebert, T. \& Schehl, J. (2013). Statistik: eine verständliche Einführung (2., überarb. Aufl.). Wiesbaden: Springer VS. 
Kuper, H. \& Kapelle, N. (2012). Lehrerkooperation aus organisationssoziologischer Sicht. In E. Baum, T.-S. Idel \& H. Ullrich (Hrsg.), Kollegialität und Kooperation in der Schule. Theoretische Konzepte und empirische Befunde (S. 41-51). Wiesbaden: VS Verlag für Sozialwissenschaften.

Lautenschläger, T. (2014). Zusätzliches Personal als Gelingensbedingung für Integration und Inklusion? Erfahrungsbericht aus dem Alltag eines Integrationshelfers/Schulbegleiters. In V. Blum \& E. Diegelmann (Hrsg.), So kann Inklusion an Schulen gelingen! Praxisberichte aus unterschiedlichen Perspektiven (Grundkurs Schulmanagement, Bd. 6, S. 82-84). Kronach: Link.

Lee, I. A. \& Preacher, K. J. (2013). Calculation for the test of the difference between two dependent correlations with one variable in common [Computer software]. Verfügbar unter http://quantpsy.org/corrtest/corrtest2.htm [Abgerufen am 30.05.2016].

Lehmann-Wermser, A., Naacke, S., Nonte, S. \& Ritter, B. (Hrsg.). (2010). Musisch-kulturelle Bildung an Ganztagsschulen. Empirische Befunde, Chancen und Perspektiven (Studien zur ganztägigen Bildung). Weinheim: Juventa.

Lelgemann, R., Singer, P., Walter-Klose, C. \& Lübbeke, J. (2012). Qualitätsbedingungen schulischer Inklusion für Kinder und Jugendliche mit dem Förderschwerpunkt Körperliche und motorische Entwicklung. Zeitschrift für Inklusion (4).

Lindmeier, B., Polleschner, S. \& Thiel, S. (2014). Schulassistenz in der Region Hannover-Bericht zur Fachtagung „Rolle der Schulassisten z in inklusiven Grundschulen“ am 25.4.2014. Verfügbar unter http://www.hannover.de/Media/01-DATA-Neu/Downloads/Region-Hannover/Soziales/Rolle-der-Schulassistenzin-inklusiven-Grundschulen [Abgerufen am 30.05.2016].

Lindmeier, B. \& Polleschner, S. (2014). Schulassistenz - ein Beitrag zu einer inklusiven Schule oder zur Verfestigung derzeitiger Schulstrukturen? Gemeinsam leben, 22 (4), 195-205.

Loos, S. (2014). Assistenz in der Schule ist Teil des Menschenrechts auf Bildung. Rechtliche Grundlagen und (menschen)rechtlicher Anspruch. In U. Barth \& T. Maschke (Hrsg.), Inklusion - Vielfalt gestalten. Ein Praxisbuch (1. Aufl., S. 496-508). Stuttgart: Verl. Freies Geistesleben.

Lortie, D. C. (1975). Schoolteacher. A sociological study (Phoenix Books, Bd. 748). Chicago: Phoenix.

Lossen, K., Rollett, W. \& Willems, A. S. (2013). Organisationskulturelle Bedingungen auf der Schulebene: Zur Beziehung von innerschulischer Kooperation, Beanspruchungserleben und Innovationsbereitschaft in Lehrerkollegien an Ganztagsgrundschulen. Zeitschrift für Grundschulforschung, 6 (2), 3852.

Lübeck, A. (2016a). „Wenn man nicht integriert ist an der Schule, kann man auch nicht als Integrationshelfer arbeiten." - Spannungsfelder zum Einsatz von Schulbegleitungen aus wissenschaftlicher Perspektive. Dialog Erriehungshilfe (1), 46-50.

Lübeck, A. (2016b). Schulbegleitungen bewusst gestalten - Schule in der Verantwortung. Lernchancen, 19 (110/111), 40-43.

Lübeck, A. \& Heinrich, M. (2016). Schulbegleitung im Professionalisierungsdilemma. Rekonstruktionen zur inklusiven Beschulung. Münster: MV-Verlag.

Lütje-Klose, B. (2011). Inklusion - Welche Rolle kann die Sonderpädagogik übernehmen? Sonderpädagogische Förderung in NRW Mitteilungen, 49 (4), 8-21.

Lütje-Klose, B. \& Willenbring, M. (1999). Kooperation fällt nicht vom Himmel. Möglichkeiten der Unterstützung kooperativer Prozesse in Teams von Regelschullehrerin und Sonderpädagogin aus systemischer Sicht. Behindertenpädagogik, 38 (1), 2-31.

Malecki, A. (2013). Sonderpädagogische Förderung in Deutschland - eine Analyse der Datenlage in der Schulstatistik (Statistisches Bundesamt, Hrsg.). Verfügbar unter https://www.destatis.de/DE/Publikationen/WirtschaftStatistik/BildungForschungKultur/SonderpaedagogischeFoerderung_52013.pdf?_blob=publicationFile [Abgerufen am 30.05.2016]. 
Marvin, C. A. (1990). Problems in school-based spreech-language consultation and collaboration services: Defining the terms and improving the process. In W. A. Secord \& E. H. Wiig (Eds.), Collaborative programs in the schools. Concepts, models, and procedures (pp. 37-47). San Antonio, TX: Psychological Corporation.

Mays, D. (2014). Drei Freunde müsst ihr sein!? - Pädagogische Fachkräfte in der Schule stärken Teamstrukturen in inklusiven Klassen entwickeln. Jugendhilfe (1), 57-61.

Melzer, C., Hillenbrand, C., Sprenger, D. \& Hennemann, T. (2015). Aufgaben von Lehrkräften in inklusiven Bildungssystemen - Review internationaler Studien. Erziebungswissenschaft, 26 (51), 61-80.

Merten, R. \& Olk, T. (1999). Sozialpädagogik als Profession. Historische Entwicklung und künftige Perspektiven. In A. Combe \& W. Helsper (Hrsg.), Pädagogische Professionalität. Untersuchungen zum Typus pädagogischen Handelns (Suhrkamp-Taschenbuch Wissenschaft, Bd. 1230, S. 570-613). Frankfurt a. M.: Suhrkamp.

Moser, V. (Hrsg.). (2013). Die inklusive Schule. Standards für die Umsetzung (Schulpädagogik, 2. Aufl.). Stuttgart: Kohlhammer.

Moser, V. \& Demmer-Dieckmann, I. (2013). Professionalisierung und Ausbildung von Lehrkräften für inklusive Schulen. In V. Moser (Hrsg.), Die inklusive Schule. Standards für die Umsetzung (Schulpädagogik, 2. Aufl., S. 155-174). Stuttgart: Kohlhammer.

Mullis, I. V., Martin, M. O., Kennedy, A. M. \& Foy, P. (2007). PIRLS 2006 International Report. IEA's Progress in International Reading Literacy Study in primary schools in 40 countries. Boston: TIMSS \& PIRLS International Study Center. Verfügbar unter http://timss.bc.edu/PDF/p06_international_report.pdf [Abgerufen am 30.05.2016].

Niedermayer, G. (2009). Die Rolle der Integrationsbegleiter. In P. Thoma \& C. Rehle (Hrsg.), Inklusive Schule. Leben und Lernen mittendrin (S. 225-235). Bad Heilbrunn: Klinkhardt.

NLQ (2016). Integrationshelferinnen und -helfer. Verfügbar unter http://www.nibis.de/nibis.php?menid $=6011$ [Abgerufen am 30.05.2016].

OECD (2009). Creating effective teaching and learning environments. First results from TALIS. Verfügbar unter http://www.oecd-ilibrary.org/docserver/download/8709011e.pdf?ex-

pires $=1461088635 \&$ id $=$ id \&ac-

cname $=$ oid009539\&checksum $=8$ BE95F28B78F8C22E20A6C5B960EFAF0 [Abgerufen am 30.05.2016].

OECD (2014). TALIS 2013 Results. An international perspective on teaching and learning. Verfügbar unter http://www.oecd-ilibrary.org/education/talis-2013-results_9789264196261-en [Abgerufen am 30.05.2016].

Olk, T., Speck, K. \& Stimpel, T. (2011). Professionelle Kooperation unterschiedlicher Berufskulturen an Ganztagsschulen - Zentrale Befunde eines qualitativen Forschungsprojektes. Zeitschrift für Erziehungswissenschaft, 14 (3), 63-80.

Pohlmann, B. \& Möller, J. (2010). Fragebogen zur Erfassung der Motivation für die Wahl des Lehramtsstudiums (FEMOLA). Zeitschrift für Pädagogische Psychologie, 24 (1), 73-84.

Pool, S. \& Moser Opitz, E. (2014). Inklusiver Unterricht - grundsätzliche Fragen und Ergebnisse einer explorativen Studie. Empirische Sonderpädagogik (2), 133-149.

Prammer-Semmler, E. \& Prammer, W. (2014). Pädagogische Assistenz ist keine billige Lehrerarbeit! Gemeinsam leben, 22 (4), 206-218.

Prieß, T. (2009). Schulbegleitung aus rechtlicher Sicht - zur Durchführungspraxis in Niedersachsen. In E. Weber \& I. Bülow (Hrsg.), Mit Autismus muss gerechnet werden! (S. 325-335). Norderstedt: Books on Demand GmbH.

Pröbstel, C. H. \& Soltau, A. (2012). Wieso Lehrkräfte (nicht) kooperieren. Die Bedeutung „personaler Faktoren" in der Zusammenarbeit am Arbeitsplatz Schule. In E. Baum, T.-S. Idel \& H. Ullrich 
(Hrsg.), Kollegialität und Kooperation in der Schule. Theoretische Konzepte und empirische Befunde (S. 55-75). Wiesbaden: VS Verlag für Sozialwissenschaften.

Putnam, R. T. \& Borko, H. (2000). What do new views of knowledge and thinking have to say about research on teacher learning? Educational Researcher, 29 (1), 4-15.

Quellenberg, H. (2009). Studie zur Entwicklung von Ganætagsschulen (StEG) - ausgewäblte Hintergrundvariablen, Skalen und Indices der ersten Erhebungswelle (Materialien zur Bildungsforschung, Bd. 24). Frankfurt a. M.: Gesellschaft zur Förderung Pädagogischer Forschung; Deutsches Institut für Internationale Pädagogische Forschung.

Rahm, S., Rabenstein, K. \& Nerowski, C. (2015). Basiswissen Ganztagsscbule: Konzepte, Erwartungen, Perspektiven (Bildungswissen Lehramt, Bd. 28). Weinheim: Beltz.

Reh, S. \& Breuer, A. (2012). Positionierungen in interprofessionellen Teams - Kooperationspraktiken in Ganztagsschulen. In S. G. Huber \& F. Ahlgrimm (Hrsg.), Kooperation. Aktuelle Forschung zur Kooperation in und zwischen Schulen sowie mit anderen Partnern (S. 185-201). Münster: Waxmann.

Richter, D. \& Pant, H. A. (2016). Lebrerkooperation in Deutschland. Eine Studie zu kooperativen Arbeitsbeziebungen bei Lebrkeäften der Sekundarstufe I. Bertelsmann Stiftung, Robert Bosch Stiftung, Stiftung Mercator, Deutsche Telekom Stiftung. Zugriff am 11.04.2016. Verfügbar unter http://www.bosch-stiftung.de/content/language1/downloads/Studie_Lehrerkooperation_in_Deutschland.pdf

RKI (2015). Gesundheit in Deutschland. Gesundheitsberichterstattung des Bundes. Verfügbar unter https://www.rki.de/DE/Content/Gesundheitsmonitoring/Gesundheitsberichterstattung/GesInDtld/gesundheit_in_deutschland_2015.pdf?_blob=publicationFile [Abgerufen am 30.05.206].

Rolff, H.-G. (1980). Soziologie der Schulreform. Theorien, Forschungsberichte, Praxisheratung (Veröffentlichungen der Arbeitsstelle für Schulentwicklungsforschung (AFS) der Universität Dortmund). Weinheim: Beltz.

Rolff, H.-G. (2010). Schulentwicklung als Trias von Organisations-, Unterrichts- und Personalentwicklung. In T. Bohl, W. Helsper, H. G. Holtappels \& C. Schelle (Hrsg.), Handbuch Schulentwicklung: Theorie, Forscbungsbefunde, Entwicklungsprozesse, Methodenrepertoire (UTB, S. 29-36). Bad Heilbrunn: Klinkhardt.

Rothland, M. (2009). Lehrerberuf und Lehrerrolle. In S. Blömeke, T. Bohl, L. Haag, G. Lang-Wojtasik \& W. Sacher (Hrsg.), Handbuch Schule. Theorie - Organisation - Entwicklung (UTB Schulpädagogik, Bd. 8392, S. 494-502). Bad Heilbrunn: Klinkhardt.

Rudow, B. (1995). Die Arbeit des Lehrers. Zur Psychologie der Lehrertätigkeit, Lehrerbelastung und Lehrergesundheit (1. Aufl., 1. Nachdr.). Bern: Huber.

Schaarschmidt, U. (2005). Halbtagsjobber? Psychische Gesundheit im Lehrerberuf - Analyse eines veränderungsbedürftigen Zustandes (2. Aufl.). Weinheim: Beltz.

Schaarschmidt, U. (2006). Lehrerbelastung. In D. H. Rost (Hrsg.), Handwörterbuch Pädagogische Psychologie (Schlüsselbegriffe, 3., überarb. u. erw. Aufl., S. 371-378). Weinheim: Beltz PVU.

Scheerens, J., Glas, C. \& Thomas, S. M. (2003). Educational evaluation, assessment, and monitoring. A systemic approach (Contexts of learning, vol. 13). Lisse u. a.: Swets \& Zeitlinger.

Schmitz, E. \& Leidl, J. (1999). Brennt wirklich aus, wer entflammt war? Studie 2: Eine LISREL-Analyse zum Burnout-Prozeß bei Lehrpersonen. Psychologie in Erziehung und Unterricht, 45 (4), 129-145.

Schmitz, G. S. \& Schwarzer, R. (2000). Selbstwirksamkeitserwartung von Lehrern. Längsschnittbefunde mit einem neuen Instrument. Zeitschrift für Pädagogische Psychologie, 14 (1), 12-25.

Schneider, W. (2004). Schulische Kooperation - ein Weg zur Integration? Eine empirische Analyse der Integration durch Kooperation von Volksschule und Schule zur Sprachförderung, Kooperationsklassen in Dachau (Schriften zur Sprachheilpädagogik, Bd. 11). Berlin: Edition Marhold im Wissenschaftsverlag Volker Spiess.

Schnell, I. (2014). Interview mit Dr. Valentin Aichele. Gemeinsam leben, 22 (4), 233-238.

Schnell, R., Hill, P. B. \& Esser, E. (2013). Methoden der empirischen Sozialforschung (10., überarb. Aufl.). München: Oldenbourg. 
Schöler, J. (2002). „Neben ihr sitzt immer ein Erwachsener“ - die Tätigkeiten von pädagogischen Hilfskräften im gemeinsamen Unterricht von behinderten und nichtbehinderten Kindern. Gemeinsam leben, 10 (4), 161-165.

Scholz, D. (2012). Lehrer in neuen Rollen. Vom Hauptdarsteller zum Prozessbegleiter. In S. Stangier \& E.-M. Thoms (Hrsg.), Eine Scbule für alle. Inklusion umsetzen in der Sekundarstufe (S. 39-44). Mülheim an der Ruhr: Verlag an der Ruhr.

Schuster, N. (2013). Schüler mit Autismus-Spektrum-Störungen. Eine Innen- und Außenansicht mit praktischen Tipps für Lehrer, Psychologen und Eltern (3., akt. u. erw. Aufl.). Stuttgart: Kohlhammer.

Schütze, F. (1999). Organisationszwänge und hoheitsstaatliche Rahmenbedingungen im Sozialwesen: Ihre Auswirkung auf die Paradoxien des professionellen Handelns. In A. Combe \& W. Helsper (Hrsg.), Pädagogische Professionalität. Untersuchungen zum Typus pädagogischen Handelns (Suhrkamp-Taschenbuch Wissenschaft, Bd. 1230, S. 183-275). Frankfurt a. M.: Suhrkamp.

Schwarz, K. (2012). Zur Beantragung einer Schulbegleitung für Schülerinnen und Schüler mit autistischem Verhalten. In H. Sautter, K. Schwarz \& R. Trost (Hrsg.), Kinder und Jugendliche mit AutismusSpektrum-Störung. Neue Wege durch die Schule (S. 229-242). Stuttgart: Kohlhammer.

Schwarzer, R. \& Jerusalem, M. (Hrsg.). (1999). Skalen zur Erfassung von Lehrer- und Schülermerkmalen. Dokumentation der psychometrischen Verfahren im Rahmen der Wissenschaftlichen Begleitung des Modellversuchs Selbstwirksame Schulen. Berlin: Freie Universität Berlin.

Schwarzer, R. \& Warner, L. M. (2014). Forschung zur Selbstwirksamkeit bei Lehrerinnen und Lehrern. In E. Terhart, H. Bennewitz \& M. Rothland (Hrsg.), Handbuch der Forscbung zum Lehrerberuf (2., überarb. u. erw. Aufl., S. 662-678). Münster: Waxmann.

Sedlmeier, P. \& Renkewitz, F. (2013). Forschungsmethoden und Statistik für Psychologen und Sozialwissenschaftler (2., akt. u. erw. Aufl.). München: Pearson.

Soltau, A., Berthe, S. \& Mienert, M. (2012). Das Autonomie-Paritäts-Muster. Der Lehrer im Spannungsfeld von kollegialer Norm und Entwicklungsanspruch. In S. G. Huber \& F. Ahlgrimm (Hrsg.), Kooperation. Aktuelle Forschung zur Kooperation in und zwischen Schulen sowie mit anderen Partnern (S. 89-102). Münster: Waxmann.

Speck, K., Olk, T. \& Stimpel, T. (2011a). Auf dem Weg zu multiprofessionellen Organisationen? Die Kooperation von Sozialpädagogen und Lehrkräften im schulischen Ganztag. Empirische Befunde aus der Ganztagsforschung und dem Forschungsprojekt „Professionelle Kooperation von unterschiedlichen Berufskulturen an Ganztagsschulen“ (ProKoop). In W. Helsper \& R. Tippelt (Hrsg.), Pädagogische Professionalität. (Zeitschrift für Pädagogik, 57, Beiheft, S. 184-201). Weinheim: Beltz.

Speck, K., Olk, T. \& Stimpel, T. (2011b). Professionelle Kooperation unterschiedlicher Berufskulturen an Ganztagsschulen - Zwischen Anspruch und Wirklichkeit. In K. Speck, T. Olk, O. Böhm-Kasper, H.-J. Stolz \& C. Wiezorek (Hrsg.), Ganఇtagsschulische Kooperation und Professionsentwicklung. Studien zu multiprofessionellen Teams und sozialräumlicher Vernetzung (Studien zur ganztägigen Bildung, S. 7-28). Weinheim: Juventa.

Speck, O. (1988). System Heilpädagogik. Eine ökologisch reflexive Grundlegung. München: Ennst Reinhardt.

Spieß, E. (2004). Kooperation und Konflikt. In H. Schuler (Hrsg.), Organisationspsychologie. Gruppe und Organisation (Enzyklopädie der Psychologie, Themenbereich D Praxisgebiete, Serie III Wirtschafts-, Organisations- und Arbeitspsychologie, Bd. 4, S. 193-250). Göttingen: Hogrefe.

StB (Hrsg.) (2012). Statistiken der Kinder- und Jugendhilfe. Errieherische Hilfe, Eingliederungshilfe für seelisch behinderte junge Menschen, Hilfe für junge Volljäbrige. Eingliederungshilfen nach \35a SGB VIII 2010. Verfügbar unter https://www.destatis.de/GPStatistik/servlets/MCRFileNodeServlet/DEHeft_derivate_00014874/5225101107004.pdf [Abgerufen am 30.05.2016].

StB (Hrsg.) (2013). Statistik der Sozialbilfe. Eingliederungshilfe für behinderte Menschen 2010. Verfügbar unter https://www.destatis.de/DE/Publikationen/Thematisch/Soziales/Sozialhilfe/Eingliederungshilfe_Behinderte5221301107004.pdf?_blob=publicationFile [Abgerufen am 30.05.2016]. 
StB (Hrsg.) (2015a). Statistik der Sozialhilfe. Eingliederungshiffe für behinderte Menschen 2013. Verfügbar unter https://www.destatis.de/DE/Publikationen/Thematisch/Soziales/Sozialhilfe/Eingliederungshilfe_Behinderte5221301137004.pdf?_blob=publicationFile [Abgerufen am 30.05.2016].

StB (Hrsg.) (2015b). Statistiken der Kinder- und Jugendhilfe. Erzieherische Hiffe, Eingliederungshilfe für seelisch bebinderte junge Menschen, Hilfe für junge Volljäbrige. Eingliederungshilfen nach \ 35 a SGB VIII 2013. Verfügbar unter https://www.destatis.de/DE/Publikationen/Thematisch/Soziales/KinderJugendhilfe/ErzieherischeHilfeEingliederungshilfe5225118137004.pdf?_blob=publicationFile [Abgerufen am 30.05.2016].

StB (2016). Allgemeinbildende und berufliche Schulen. Scbüler/innen nach Schularten. Verfügbar unter https://www.destatis.de/DE/ZahlenFakten/GesellschaftStaat/BildungForschungKultur/Schulen/Tabellen/AllgemeinBildendeBeruflicheSchulenSchulartenSchueler.html [Abgerufen am 30.05.2016].

Steiner, C. (2013). Die Einbindung pädagogischer Laien in den Alltag von Ganztagsschulen. Bildungsforschung, 10 (1), 64-90.

Steiner, C. \& Tillmann, K. (2011). Koordinierte Vielfalt? Über die Arbeit in multiprofessionellen Ganztagsteams. In K. Speck, T. Olk, O. Böhm-Kasper, H.-J. Stolz \& C. Wiezorek (Hrsg.), Ganætagsschulische Kooperation und Professionsentwicklung. Studien zu multiprofessionellen Teams und sozialräumlicher Vernetzung (Studien zur ganztägigen Bildung, S. 48-68). Weinheim: Juventa.

Steinert, B., Harting, J. \& Klieme, E. (2008). Institutionelle Bedingungen der Sprachkompetenzen. In E. Klieme, W. Eichler, A. Helmke, R. H. Lehmann, G. Nold, H.-G. Rolff et al. (Hrsg.), Unterricht und Kompetenzerwerb in Deutsch und Englisch. Ergebnisse der DESI-Studie (S. 411-450). Weinheim: Beltz.

Steinert, B., Klieme, E., Maag Merki, K., Döbrich, P., Halbheer, U. \& Kunz, A. (2006). Lehrerkooperation in der Schule: Konzeption, Erfassung, Ergebnisse. Zeitschrift für Pädagogik, 52 (2), 185-204.

Stivers, J. \& Cramer, S. (2015). New metaphors for teacher/paraeducator relationships. In D. Chambers (Eds.), Working with teaching assistants and other support staff for inclusive education (International perspectives on inclusive education, vol. 4, pp. 27-47). Bingley (UK): Emerald.

Takala, M. (2007). The work of classroom assistants in special and mainstream education in Finland. British Journal of Special Education, 34 (1), 50-57.

Terhart, E. (Hrsg.) (1991). Unterrichten als Beruf. Neuere amerikanische und englische Arbeiten zur Berufskultur und Berufsbiographie von Lebrern und Lebrerinnen (Studien und Dokumentationen zur vergleichenden Bildungsforschung, Bd. 50). Köln: Böhlau.

Terhart, E. (1999). Berufskultur und professionelles Handeln bei Lehrern. In A. Combe \& W. Helsper (Hrsg.), Pädagogische Professionalität. Untersuchungen zum Typus pädagogischen Handelns (Suhrkamp-Taschenbuch Wissenschaft, Bd. 1230, S. 448-471). Frankfurt a. M.: Suhrkamp.

Thies, W. (2014). Assistenz für wen, was und wie? Von der Einzelbegleitung zum gruppenbezogenen Einsatz im multiprofessionellen Klassenteam. In S. Trumpa, S. Seifried, E. Franz \& T. Klauß (Hrsg.), Inklusive Bildung: Erkenntnisse und Konzepte aus Fachdidaktik und Sonderpädagogik (S. 234-243). Weinheim: Juventa.

Tillmann, K. \& Rollett, W. (2011). Multiprofessionelle Kooperation und Partizipation an Ganztagsschulen - Welche Auswirkung hat die strukturelle Einbindung des weiteren pädagogisch tätigen Personals auf die berufsgruppenübergreifende Zusammenarbeit? In K. Speck, T. Olk, O. Böhm-Kasper, H.-J. Stolz \& C. Wiezorek (Hrsg.), Ganztagsscbulische Kooperation und Professionsentwicklung. Studien zu multiprofessionellen Teams und sozialräumlicher Vernetzung (Studien zur ganztägigen Bildung, S. 29-47). Weinheim: Juventa.

Tillmann, K. \& Willems, A. S. (2016). Zwischen Selbstverständlichkeit und seltenem Event: Typische Muster multiprofessioneller Kooperation an Ganztagsschulen im Kontext schulischer Struktur- und Prozessqualität. Vortrag auf der 25. Tagung der Deutschen Gesellschaft für Erziehungswissenschaft (DGfE), Universität Kassel.

Ulich, K. (1996). Beruf: Lehrer/in. Arbeitsbelastungen, Beriehungskonflikte, Zufriedenheit. Weinheim: Beltz. 
Van Santen, E. \& Seckinger, M. (2003). Kooperation: Mythos und Realität einer Praxis. Eine empirische Studie zur interinstitutionellen Zusammenarbeit am Beispiel der Kinder- und Jugendhilfe. München: Deutsches Jugendinstitut.

Villegas-Reimers, E. (2003). Teacher professional development. An international review of the literature. Paris: International Institute for Educational Planning.

Webster, R., Blatchford, P., Bassett, P., Brown, P., Martin, C. \& Russell, A. (2010). Double standards and first principles. Framing teaching assistant support for pupils with special educational needs. European Journal of Special Needs Education, 25 (4), 319-336.

Webster, R., Blatchford, P., Bassett, P., Brown, P., Martin, C. \& Russell, A. (2011). The wider pedagogical role of teaching assistants. School leadership \& management, 31 (1), 3-20.

Webster, R., Blatchford, P. \& Russell, A. (2013a). Challenging and changing how schools use teaching assistants. Findings from the Effective Deployment of Teaching Assistants Project. School Leadership \& Management, 33 (1), 78-96.

Webster, R., Russell, A. \& Blatchford, P. (2013b). Teaching assistants. A guide to good practice (Oxford University Press, Ed.). Verfügbar unter http://fdslive.oup.com/www.oup.com/oxed/primary/literacy/osi_teaching_assistants_report_web.pdf?region=international [Abgerufen am 30.05.2016].

Weick, K. E. (1976). Educational organizations as loosely coupled systems. Administrative Science Quarterly, 21 (1), 1-19.

Wessel, J. (2005). Kooperation im gemeinsamen Unterricht. Die Zusammenarbeit von Lehrern in der schulischen Integration hörgeschädigter Kinder und Jugendlicher. Münster: Monsenstein und Vannerdat.

Wiater, W. (2009). Zur Definition und Abgrenzung von Aufgaben und Funktionen der Schule. In S. Blömeke, T. Bohl, L. Haag, G. Lang-Wojtasik \& W. Sacher (Hrsg.), Handbuch Schule. Theorie - Organisation - Entwicklung (UTB Schulpädagogik, Bd. 8392, S. 65-72). Bad Heilbrunn: Klinkhardt.

Wilson, S. M. \& Berne, J. (1999). Teacher learning and the acquisition of professional knowledge: An examination of research on contemporary professional development. Review of Research in Education, 24, 173-209.

Wohlgemuth, K. (2009). Schulbegleitung in Thüringen. Rabmenbedingungen, Aufgaben und Belastungen. Unveröffentlichte Diplomarbeit, Friedrich-Schiller-Universität Jena.

Zauner, M. \& Zwosta, M. (2014). Effektestudie zu Schulbegleitungen. Technische Hochschule Regensburg. Verfügbar unter http://www.kjf-regensburg.de/documents/10502/1053779/Effektestudie10.11.14.pdf/38f5880c-ea96-4aff-895f-b298bf59d6c9 [Abgerufen am 30.05.2016].

Zierer, K., Speck, K. \& Moschner, B. (2013). Methoden erziehungswissenschaftlicher Forschung (utb-studi-ebook, Bd. 4026). München: Reinhardt. 


\section{Abbildungsverzeichnis}

Abb. 1: Kooperationsmodell von Fussangel (2008) .35

Abb. 2: Arbeitsmodell zur Kooperation von Klassenleitung und Schulbegleitung.......

Abb. 3: Entwicklung der Bewilligungszahlen von Schulbegleitungen durch das Jugendamt der Stadt und das Sozialamt des Landkreises Göttingen

Abb. 4: Anteilige Verteilung der Schulbegleiter/innen nach höchstem einschlägigen formalen

Bildungsabschluss

Abb. 5: Häufigkeiten der Zeitangaben zur bisherigen Beschäftigungsdauer als Schulbegleitung und zur gewünschten weiteren Beschäftigungsdauer

Abb. 6: Häufigkeiten der Zeiträume der Gesamtlaufzeit des aktuellen Vertrags als Schulbegleitung.....

Abb. 7: Häufigkeiten der Zeitangaben zur bisherigen Tätigkeit für den aktuell begleiteten Schüler oder die aktuell begleitete Schülerin

Abb. 8: Bewilligendes Amt der Maßnahme und Angabe der Behinderung der Schüler/innen durch die Schulbegleiter/innen .69

Abb. 9: Verteilung der Schulbegleitungsmaßnahmen über die Schulformen ...............................................70

Abb. 10: Häufigkeiten der jeweiligen Wochenstunden der Schulbegleitung

Abb. 11: Prozentuale Nennung der Häufigkeit der Ausübung von Tätigkeiten im Rahmen von Schulbegleitung .72

Abb. 12: Verteilung der Schulbegleitungen über die Jugendämter von Stadt und Landkreis Göttingen und über die Sozialämter von Stadt und Landkreis Göttingen auf die Schulformen .74

Abb. 13: Prozentuale Anteile der Austauschhäufigkeiten von Schulbegleiter/innen mit bestimmten 


\section{Tabellenverzeichnis}

Tab. 1: Zuordnung der Fragebogenitems zum Tätigkeitsprofil zu verschiedenen Aufgabenbereichen .......57

Tab. 2: Itemkennwerte und Skalenkennwerte zur Selbstwirksamkeitserwartung und zur

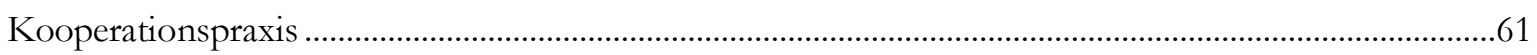

Tab. 3: Itemkennwerte und Skalenkennwerte zum arbeitsbezogenen Erleben.............................................62

Tab. 4: Mittelwerte der Angaben zur Bewertung des Austauschs mit verschiedenen Personengruppen .....77

Tab. 5: Korrelationskoeffizienten für den Zusammenhang von Kooperationsformen und

Kooperationswirkungen .78 


\section{Abkürzungsverzeichnis}

ADHS

AGJ

DESI

DRK

IGLU

JSN

KMK

MUKUS

NLQ

NSchG

OECD

PIRLS

PISA

QuaSI

RKI

SGB

$\mathrm{StB}$

StEG

TALIS

UN

WHO
Aufmerksamkeitsdefizit-/Hyperaktivitätsstörung

Arbeitsgemeinschaft für Kinder- und Jugendhilfe

Schulleistungsstudie Deutsch Englisch Schülerleistungen International (deutsche Bezeichnung der PIRLS-Studie, siehe unten)

Deutsches Rotes Kreuz

Schulleistungsstudie Internationale Grundschul-Lese-Untersuchung

Jugendhilfe Südniedersachsen e. V.

Kultusministerkonferenz

Studie zur musisch-kulturellen Bildung an Ganztagsschulen

Niedersächsisches Landesinstitut für schulische Qualitätsentwicklung

Niedersächsisches Schulgesetz

Organisation for Economic Co-operation and Development/Organisation für wirtschaftliche Zusammenarbeit und Entwicklung

Schulleistungsstudie Progress in International Reading Literacy Study der International Association for the Evaluation of Educational Acbievement (IEA)

Schulleistungsstudie Programme for International Student Assessment der OECD

Modellprojekt zur Qualifizierung von Schulbegleitern und Schaffung von Net-iverken für die gelungene schulische Integration in Thüringen

Robert-Koch-Institut

Sozialgesetzbuch

Statistisches Bundesamt

Studie zur Entwicklung von Gan§tagsschulen

Schulstudie Teaching and Leaming International Survey der OECD

United Nations/Vereinte Nationen

World Health Organisation/Weltgesundheitsorganisation 


\title{
Göttinger Beiträge zur erziehungswissenschaftlichen Forschung
}

\author{
Herausgeber_innen \\ für das Institut für Erziehungswissenschaft der Universität Göttingen: \\ Prof. Dr. Klaus-Peter Horn, Prof. Dr. Katharina Kunze \\ Prof. Dr. Kerstin Rabenstein, Prof. Dr. Tobias C. Stubbe, \\ Prof. Dr. Hermann Veith, Prof. Dr. Ariane S. Willems
}

Die Göttinger Beiträge zur erziehungswissenschaftlichen Forschung wurden 1991 gegründet, um für kleinere Studien und explorative empirische Arbeiten, Dokumentationen und Forschungsberichte von Mitarbeiter_innen des damaligen Pädagogischen Seminars sowie für sehr gute studentische Abschlussarbeiten eine Publikationsmöglichkeit zu schaffen. Auch nach der Umbenennung des Pädagogischen Seminars in Institut für Erziehungswissenschaft wird die Reihe fortgesetzt. Ab Heft 34 erscheinen die „Göttinger Beiträge zur erziehungswissenschaftlichen Forschung“ als open access-Publikation auf den Seiten des Universitätsverlags Göttingen (http://www.univerlag.uni-goettingen.de).

Einzelne Restexemplare früherer Ausgaben sind weiterhin zum Versandkostenpreis erhältlich oder können bei Bedarf gegen eine geringe Gebühr eingescannt werden. Bitte wenden Sie sich hierzu an das Institut für Erziehungswissenschaft der Universität Göttingen (ife@gwdg.de).

Bisher erschienene Ausgaben:

1

Günter Schreiner

Undemokratischer Schulalltag? Vergleichende Erkundungen zum Schul-Erleben in Ost- und Westdeutschland.

44 Seiten, 1991

2

Jörg Schlömerkemper

Freizeit und Karriere. Freizeitaktivitäten von Kindern im Urteil von Erwachsenen - Eine empirische Studie.

37 Seiten, 1991

3

Christian Rittelmeyer

Schulbau-Architektur. Über die Wirkungen von Bauformen und Raumfarben auf Schülerinnen und Schüler.

50 Seiten, 1991

4

Thomas Harms, Ralf Tarant und Jörg Schlömerkemper

Pädagogik-Studium und Beruf. Eine empirische Studie zum Magister-Studiengang am Göttinger Pädagogischen Seminar.

79 Seiten, 1992

5

Cornelie Dietrich, Klaus Mollenhauer

Untersuchungen zum Musik-Verstehen bei 9-12jährigen Kindern.

62 Seiten, 1992

\section{$6 / 7$}

Helga Hauenschild, Hans-Georg Herrlitz und Birgit Kruse

Die Lehrgestalt der westdeutschen Erziehungswissenschaft von 1945 bis 1990 (LEWERZ).

98 Seiten plus Anhang, 1993 


\section{Christian Rittelmeyer}

Empirische Untersuchungen zur Wahrnehmung von Schulbauten. Zwei Untersuchungen zur Faktorenstruktur und Sensomotorik der Schulbauwahrnehmung.

59 Seiten, 1994

$9 / 10$

Gustava Schefer-Vietor, Jörg Schlömerkemper, Wulf Hopf

Geschlecht und Naturwissenschaften (GENUS). Eine empirische Studie über Mädchen und Jungen im Physik-Unterricht am Gymnasium.

160 Seiten, 1994

11

Wulf Hopf unter Mitarbeit von Hendrik Abel und Nils Pagels

Fremdenfeindlichkeit und Ökonomismus von Jugendlichen. Befunde einer qualitativen Studie.

57 Seiten, 1995

12

\section{Edeltraud Dubbi}

Fortbildungsorientierte Supervision in der Altenpflege.

70 Seiten, 1995

\section{3}

\section{Klaus Mollenhauer}

Sozialpädagogische Praxis, Forschung und Theorie - Drei einführende Versuche.

72 Seiten, 1997

14

Christian Rittelmeyer

Der urteilende Leib. Empirische Materialien zu einer pädagogischen Ästhesiologie.

57 Seiten, 1997

\section{5}

Christina Krause unter Mitarbeit von Verena Meyer, Christel Rohde, Christine Stückle und Renate-Katrin Zimmermann

Ich bin Ich - Gesundheitsförderung durch Selbstwertstärkung. Bericht über ein Projekt zur Gesundheitsförderung in Grundschulen.

78 Seiten, 1998

16

Krischna Gutschke, Gunda Schröder und Jörg Schlömerkemper

Interaktion mit „Behinderungen“. Eine empirische Studie am Beispiel der Interaktion zwischen gut-hörenden und schwer-hörenden Personen.

67 Seiten, 1998

17

Doris Lemmermöhle/Gustava Schefer-Viëtor (Hrsg.)

Geschlechterspannung.

58 Seiten, 1999

18

Dominik Petko

Handlungsleitende Orientierungen von sozialpädagogischen FamilienhelferInnen.

78 Seiten, 1999 

Environment for Schools. An International Perspective.

47 Seiten, 2000

\section{0}

\section{Claudia Göhmann, Robert Kreitz, Maike Lambrecht}

Das Studium und die Lehre am Pädagogischen Seminar aus der Sicht der Studierenden. Ergebnisse einer Befragung.

70 Seiten, 2000

\section{1}

\section{Peter Alheit, Morten Brandt, Hans-Rüdiger Müller, Frank Schömer}

Konfigurationen der Bildung. Drei Fallstudien zur Leibthematik im autobiographischen Text um 1800.

83 Seiten, 2001

\section{2}

\section{Margarete Menz}

Schulalltag und Geschlecht. Eine empirische Studie zu Geschlechterkonstruktionen in der 5. Klasse.

102 Seiten, 2001

\section{3}

\section{Inga Truschkat}

„Meine Eltern sind beide keine Akademiker“. Herkunftsbedingungen und habituelle Logiken von Studierenden als Reproduktionsverfahren sozialer Ungleichheit. Eine biographieanalytische Untersuchung.

88 Seiten, 2002

\section{4}

\section{Anna Verschüren}

Interessenentwicklung bei jugendlichen Spätaussiedlerinnen. Pädagogische Praxisentwicklung in der außerschulischen Freizeitbetreuung.

105 Seiten, 2003

\section{5}

\section{Sara Klüsche}

Kreative Momente und Widerstand. Pilotstudie zur Prozessevaluation einer kunstdidaktischen Exkursion.

101 Seiten, 2004

\section{6}

\section{Manuela Kaiser}

Bildung und Geschlecht. Eine biographieanalytische Untersuchung der Konstruktion von Geschlecht in Studentinnenbiographien.

94 Seiten, 2004

\section{7}

\section{Maren Mareile Assmann}

Beratung - eine Profession der Zukunft? Entwicklung, Probleme und Chancen in Deutschland. 118 Seiten, 2007 


\section{Tina Pflüger, Rabea Lucht}

Berufliche Orientierungsprobleme von Studierenden der Sozialwissenschaften. Ein Vergleich von zwei Beratungsmodellen.

211 Seiten, 2008

29

Anissa Henze

„Das war also wieder das alte Schema ...“ Zum Verhältnis von Reflexivität und Autonomie in biographischen Lernprozessen. Eine biographietheoretische Untersuchung am Beispiel von „nicht-traditionellen“ Studierenden.

131 Seiten, 2008

30

Ann-Cathrin Duve

Brauchen Kinder Bücher? Lesen im medialen Zeitalter.

88 Seiten, 2008

31

Jan-Michael Andres

Depression im Kindes- und Jugendalter. Möglichkeiten und Grenzen der Einflussnahme durch Lehrer.

75 Seiten, 2010

32

Torben Lüth

Klaus Mollenhauer - ein Kritischer Pädagoge?

82 Seiten, 2010

33

\section{Christina Krause}

Wie erfolgreich ist Gesundheitsförderung in Bildungseinrichtungen? 63 Seiten, 2012

\section{4}

\section{Hans-Georg Herrlitz}

Der Streit um die Gesamtschule in Niedersachsen. Ein dokumentarischer Rückblick auf die Landtagsdebatten 1967 - 2012.

41 Seiten, 2013

35

Saskia Petschnik

Zum Potential kognitiv-empirischer Forschung für die Pädagogik. Veranschaulicht am Beispiel des Erstspracherwerbs.

59 Seiten, 2013

\section{6}

Johanna Brauns, Thomas Göymen-Steck, Klaus-Peter Horn

Lernziele, Veranstaltungs- und Prüfungsformen in erziehungswissenschaftlichen Bachelorstudiengängen. Eine vergleichende Analyse von Studienprogrammen an acht Universitäten.

69 Seiten, 2015 
eISSN: $2198-2384$

DOI: $10.17875 /$ gup2017-1029 